\title{
Variedades de Gelfand-Tsetlin
}

\author{
Germán Alonso Benitez Monsalve
}

\author{
TESE APRESENTADA \\ AO \\ InstituTo DE MATEMÁTICA E EsTATÍSTICA \\ DA \\ Universidade de SÃo PAULO \\ PARA \\ OBTENÇÃO DO TÍTULO \\ $\mathrm{DE}$ \\ DOUTOR EM CIÊNCIAS
}

\section{Programa: Doutorado em Matemática \\ Orientador: Prof. Dr. Vyacheslav Futorny}

Durante o desenvolvimento deste trabalho o autor recebeu auxílio financeiro da CAPES

São Paulo, Novembro de 2016 



\section{Variedades de Gelfand-Tsetlin}

Esta versão da tese contém as correções e alterações sugeridas pela Comissão Julgadora durante a defesa da versão original do trabalho, realizada em 21/11/2016. Uma cópia da versão original está disponível no Instituto de Matemática e Estatística da Universidade de São Paulo.

Comissão Julgadora:

- Prof. Dr. Vyacheslav Futorny (orientador) - IME-USP

- Prof. Dr. Cristian Ortiz Gonzalez- IME-USP

- Prof. Dr. Viktor Bekkert - UFMG

- Prof. Dr. Marcos Banevenuto Jardim - IMECC-UNICAMP

- Prof. Dr. Lucas Henrique Calixto - UFMG 

A mi familia:

Papá, Mamá, Suki, Henry, Fabian,

Thomas y Mathias. 



\section{Agradecimentos}

Agradeço, primeiramente a Deus, por me permitir conquistar este sonho.

Muito obrigado a minha familia pelo carinho e apoio recebido durante todos esses anos, mesmo com todas minhas doideras, são eles meus motores e minha inspiração. Ao meu pai Osvaldo Benitez Tapias e a minha mãe Fabiola Monsalve Valencia, porque desde criança me mostraram o valor de estudar. Sou grato a Suki por ser minha parceira e fazer de minhas aventuras nossas aventuras. A meus irmãos Jhon Henry e Fabian pela confiança, a meu sobrinho Thomas por me ensinar a sorrir e brincar, mesmo nos momentos mais difíceis. Também, quero agradecer a Dom Jaime Palacio Escobar, muito querido e estimado pela familia Benitez Monsalve.

Gostaria agradecer a meu orientador Vyacheslav Futorny pela paciência, amizade e confiança depositada em mim durante este projeto de pesquisa.

À banca julgadora, composta pelos profesores Vyacheslav Futorny, Cristian Ortiz Gonzalez, Viktor Bekkert, Marcos Banevenuto Jardim e Lucas Henrique Calixto, agradeço pelas sugestões dadas visando melhorar meu trabalho de tese.

Sou grato ao grupo de trabalho Luis Enrique Ramirez, Wilson Mutis e Carlos Gomes por nossos seminários sobre a pesquisa, em que apresentei boa parte deste trabalho. Também ao pessoal dos seminários sobre álgebras de Lie, em especial a Cesar Rodriguez, Carlos Payares e Elkin Quintero. Sem esquecer meu agradecimento a Kostiantyn Iusenko pelos tantos outros seminários.

Agradeço ao Programa de Pós-Graduação do Instituto de Matemática e Estatística (IME) da Universidade de São Paulo (USP) pela qualidade acadêmica e à Coordenação de Aperfeiçoamento de Pessoal de Nível Superior (CAPES), pelo apoio financeiro.

Para terminar, gostaria de agradecer aos funcionários do Instituto de Matemática e Estatística da USP pela esforço, paciência e infraestrutura oferecidas. 


\section{Resumo}

BENITEZ MONSALVE, G. A. Variedades de Gelfand-Tsetlin. 2016. Tese (Doutorado) - Instituto de Matemática e Estatística, Universidade de São Paulo, São Paulo, 2016.

Serge Ovsienko provou que a variedade de Gelfand-Tsetlin para $\mathfrak{g l}_{n}$ é equidimensional (i.e., todas suas componentes irredutíveis têm a mesma dimensão) com dimensão $\frac{n(n-1)}{2}$. Este resultado é conhecido como Teorema de Ovsienko e tem importantes consequências na Teoria de Representacões de Álgebras. Neste trabalho, provamos uma versão fraca do Teorema de Ovsienko para $\mathfrak{g l}_{n}$ e estendemos tal versão fraca a uma estrutura que tem como caso particular $\mathfrak{g l}_{3}$, esse é o caso do grupo quântico Yangian $Y_{p}\left(\mathfrak{g l}_{3}\right)$ de nível $p$. Além disso, o Teorema de Ovsienko também tem consequências na Geometria Simplética, especificamente na equidimensionalidade das fibras em uma projeção da aplicação de Kostant-Wallach. Neste trabalho apresentamos a generalização deste resultado.

Palavras-chave: Variedades algébricas, dimensão, equidimensionalidade, Gelfand-Tsetlin, KostantWallach, Yangians. 


\section{Abstract}

BENITEZ MONSALVE, G. A. Gelfand-Tsetlin varieties. 2016. Tese (Doutorado) - Instituto de Matemática e Estatística, Universidade de São Paulo, São Paulo, 2016.

Serge Ovsienko proved that the Gelfand-Tsetlin variety for $\mathfrak{g l}_{n}$ is equidimensional (i.e., all its irreducible components have the same dimension) with dimension $\frac{n(n-1)}{2}$. This result is known as Ovsienko's Theorem and it has important consequences in Representation Theory of Algebras. In this work, we prove a weak version of Ovsienko's Theorem for $\mathfrak{g l}_{n}$ and we extend that weak version to a structure which has as particular case $\mathfrak{g l}_{3}$, this case is the quantum group level $p$ Yangian $Y_{p}\left(\mathfrak{g l}_{3}\right)$. Moreover, the theorem of Ovsienko also has consequences in Symplectic Geometry, more concretely in the equidimensionality of the fibers in a projection of the Kostant-Wallach map. In this work we will present the generalization of that result.

Keywords: Algebraic varieties, dimension, equidimensionality, Gelfand-Tsetlin, Kostant-Wallach, Yangians. 


\section{Sumário}

1 Introdução 1

2 Conceitos Preliminares $\quad 5$

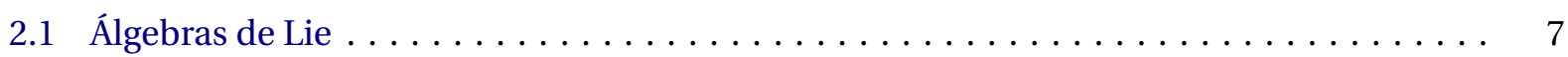

2.2 Álgebra Envolvente Universal $\ldots \ldots \ldots \ldots \ldots \ldots \ldots \ldots \ldots \ldots \ldots \ldots \ldots \ldots$

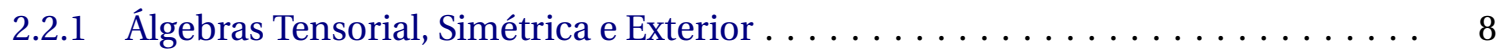

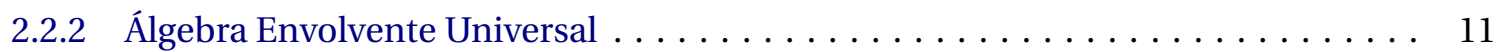

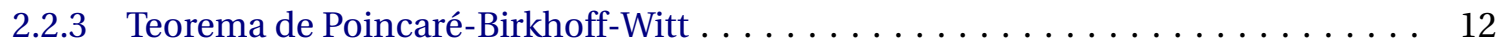

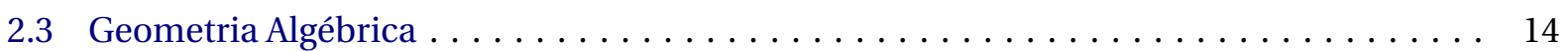

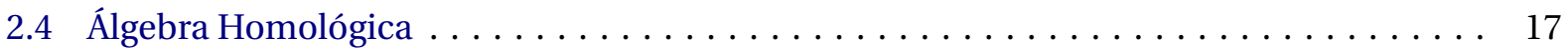

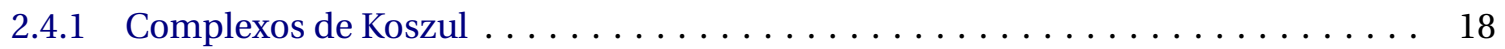

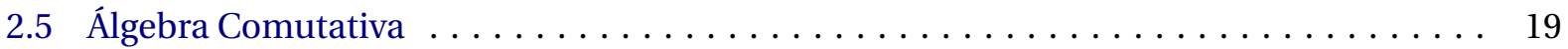

3 Teorema de Ovsienko e sua versão fraca $\quad 21$

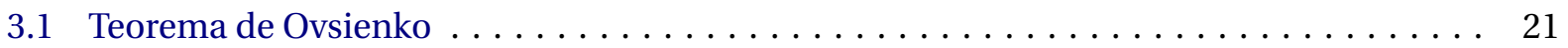

3.1.1 Subálgebra de Gelfand-Tsetlin para $\mathfrak{g l}_{n} \ldots \ldots \ldots \ldots \ldots \ldots \ldots \ldots \ldots \ldots \ldots$

3.1.2 Outros geradores da subálgebra de Gelfand-Tsetlin $\Gamma \ldots \ldots \ldots \ldots \ldots \ldots . \ldots 23$

3.1.3 Variedade de Gelfand-Tsetlin para $\mathfrak{g l}_{n} \ldots \ldots \ldots \ldots \ldots \ldots \ldots \ldots \ldots$

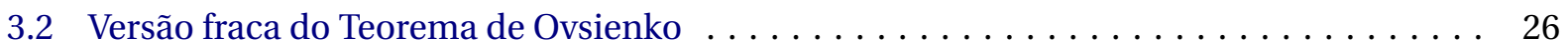

3.2.1 Casos particulares da Versão fraca do Teorema de Ovsienko . . . . . . . . . . . . 28

3.2.2 Demonstração da versão fraca do Teorema de Ovsienko $\ldots \ldots \ldots \ldots \ldots \ldots$

3.2 .3 Teorema de Kostant-Wallach $\ldots \ldots \ldots \ldots \ldots \ldots \ldots \ldots \ldots \ldots \ldots$

4 Equidimensionalidade das fibras da aplicação de Kostant-Wallach e da aplicação parcial 41

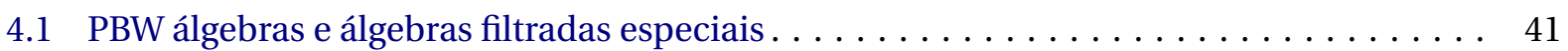

4.2 Aplicação de Kostant-Wallach e aplicação parcial de Kostant-Wallach . . . . . . . . . . . . . 44

4.3 Aplicação de Kostant-Wallach vs Variedades de Gelfand-Tseltin . . . . . . . . . . . . . . 45

4.4 Uma generalização do Teorema de Colarusso-Evens . . . . . . . . . . . . . . . . . . 46

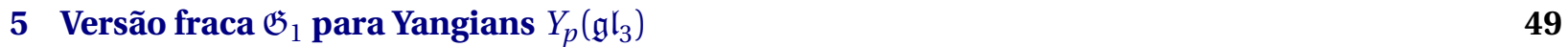

5.1 Yangians restritos $Y_{p}\left(\mathfrak{g l}_{n}\right)$ de nível $p$ para $\mathfrak{g l}_{n} \ldots \ldots \ldots \ldots \ldots \ldots \ldots \ldots \ldots$

5.1.1 Alguns polinômios da variedade de Gelfand-Tsetlin . . . . . . . . . . . . . . . 51

5.2 Variedade de Gelfand-Tsetlin para Yangians restritos $Y_{p}\left(\mathfrak{g l}_{3}\right) \ldots \ldots \ldots \ldots \ldots \ldots \ldots$

5.2.1 Equidimensionalidade para $Y_{1}\left(\mathfrak{g l}_{3}\right)$ e $Y_{2}\left(\mathfrak{g l}_{3}\right) \ldots \ldots \ldots \ldots \ldots \ldots \ldots \ldots \ldots \ldots$

5.3 Uma primeira decomposição de $\mathfrak{G}$ para Yangians restritos $Y_{p}\left(\mathfrak{g l}_{n}\right) \ldots \ldots \ldots \ldots \ldots$ 
5.3.1 Uma decomposição para $\mathfrak{G}_{1} \ldots \ldots \ldots \ldots \ldots \ldots \ldots \ldots \ldots \ldots \ldots$

5.4 Equidimensionalidade da versão fraca $\mathfrak{G}_{1}$ para $Y_{p}\left(\mathfrak{g l}_{3}\right) \ldots \ldots \ldots \ldots \ldots \ldots \ldots \ldots$

$\begin{array}{ll}\text { A Contas } & 91\end{array}$

B Decomposição da variedade de Gelfand-Tsetlin $\mathfrak{G}$ para $Y_{2}\left(\mathfrak{g l}_{3}\right) \quad 97$

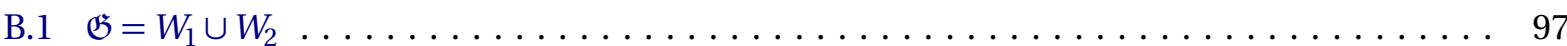

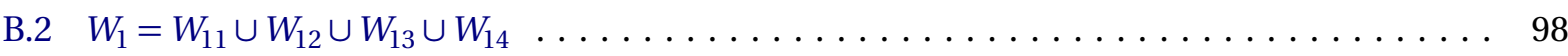

B.2.1 Decomposição de $W_{11} \ldots \ldots \ldots \ldots \ldots \ldots \ldots \ldots \ldots \ldots \ldots \ldots$

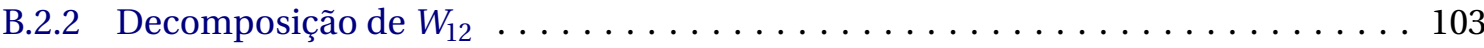

B.2.3 Decomposição de $W_{13} \ldots \ldots \ldots \ldots \ldots \ldots \ldots \ldots \ldots \ldots \ldots \ldots \ldots$

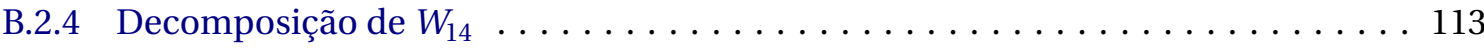

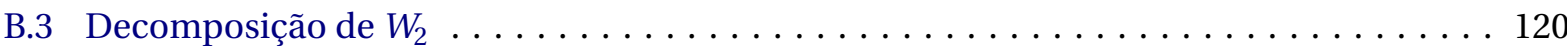

$\begin{array}{ll}\text { Referências Bibliográficas } & 125\end{array}$

$\begin{array}{ll}\text { Índice Remissivo } & 127\end{array}$ 


\section{Capítulo 1}

\section{Introdução}

Consideremos a variedade de Gelfand-Tsetlin associada à subálgebra de Gelfand-Tsetlin $\Gamma$ para a álgebra de Lie linear geral $\mathfrak{g l}_{n}$. Serge Ovsienko provou em [Ovsienko (2003)] que essa variedade é equidimensional (ou seja, todas suas componentes irredutíveis tem a mesma dimensão) com dimensão $\frac{n(n-1)}{2}$. Esse resultado é conhecido como "Teorema de Ovsienko" e, como consequência desse fato, a variedade de Gelfand-Tsetlin é uma interseção completa. De maneira independente, B. Kostant e N. Wallach em [Kostant e Wallach (2006a)] e [Kostant e Wallach (2006b)] provaram que todas as componentes regulares da variedade de Gelfand-Tsetlin para $\mathfrak{g l}_{n}$ têm dimensão $\frac{n(n-1)}{2}$.

Um famoso teorema de Kostant, afirma que a álgebra envolvente universal $U(\mathfrak{g})$ de uma álgebra de Lie semisimples e complexa $\mathfrak{g}$, é um módulo livre à esquerda (ou à direita) sobre seu centro (ver teorema $0.12 \mathrm{em}$ [Kostant (1963)]). A razão por trás desse fato é que a variedade associada ao centro de $\mathfrak{g}$ é uma interseção completa. Por exemplo, a variedade associada ao centro de $\mathfrak{g}=\mathfrak{g l}{ }_{n}$ coincide com o conjunto das matrizes nilpotentes, a qual é irredutível e possui dimensão $n^{2}-n$.

A. Molev, M. Nazarov e G. Olshanskii provaram em [Molev et al. (1996)] que o grupo quântico Yangian $Y\left(\mathfrak{g l}_{n}\right)$ é livre sobre seu centro. Também, S. Ovsienko provou em [Ovsienko (2002)] que $U\left(\mathfrak{g l}_{n}\right)$ é livre como módulo à esquerda (ou à direita) sobre sua subálgebra de Gelfand-Tsetlin.

Um resultado análogo ao teorema de Kostant em [Kostant (1963)] foi provado por V. Futorny e S. Ovsienko em [Futorny e Ovsienko (2005)] para a clase de álgebras filtradas especiais, onde eles estabelecem o seguinte resultado chave:

Teorema 1.1. Seja $U$ uma álgebra filtrada especial. Se $g_{1}, g_{2}, \ldots, g_{t} \in U$ são elementos mutuamente comutativos cujas imagens graduadas formam uma interseção completa para a álgebra graduada associada de $U$, então $U$ é livre como $k\left[g_{1}, g_{2}, \ldots, g_{t}\right]$-módulo à esquerda (ou à direita).

Como consequência desse teorema tem-se que $U(\mathfrak{g})$ e $Y\left(\mathfrak{g l}_{n}\right)$ são livres sobre seus centros e que $U\left(\mathfrak{g l}_{n}\right)$ é também livre como módulo sobre sua subálgebra de Gelfand-Tsetlin $\Gamma$. Ressaltando que esse último fato é importante pois $\Gamma$ é uma subálgebra comutativa maximal de $U\left(\mathfrak{g l}_{n}\right)$ a qual contém o centro de $U\left(\mathfrak{g l}_{n}\right)$. Usando este mesmo raciocínio, V. Futorny e S. Ovsienko conseguiram provar em [Futorny e Ovsienko (2005)] que os Yangians restritos $Y_{p}\left(\mathfrak{g l}_{n}\right)$ e a envolvente universal $U\left(\mathfrak{g}_{m}\right)$ das álgebras current $\mathfrak{g}_{m}$ são também livres sobre seus centros.

Portanto, não é estranho conjecturar que os Yangians restritos $Y_{p}\left(\mathfrak{g l}_{n}\right)$ para qualquer nível $p$, é livre sobre sua subálgebra de Gelfand-Tsetlin. V. Futorny, A. Molev e S. Ovsienko em [Futorny et al. (2005)] provaram que esta conjectura é verdadeira para $n=2$ e $p$ arbitrário.

As subálgebras de Gelfand-Tsetlin foram consideradas por A. S. Miščenko e A. T. Fomenko em [Miščenko e Fomenko (1978)] em conexões com a solução da equação de Euler. Já Ė. B. Vinberg em [Vinberg (1990)] usaram as subálgebras de Gelfand-Tsetlin em trabalhos relacionados com subálgebras de dimensão de Gelfand-Kirillov maximais da álgebra envolvente universal de uma álgebra de Lie simples. B. Kostant e N. Wallach em [Kostant e Wallach (2006a)] e [Kostant e Wallach (2006b)] usaram as subálgebras em conexão com mecânica clássica e M. I. Graev em trabalhos relacionados com funções hipergeométricas gerais sobre o grupo de Lie GL(n, $\mathbb{C})$. 
O objetivo deste trabalho é estudar a variedade associada à subálgebra de Gelfand-Tsetlin para $\mathfrak{g l}_{n}$ e $Y_{p}\left(\mathfrak{g l}_{n}\right)$ e sua equidimensionalidade. Nossa motivação vem da teoria de representações:

1. Sejam $A$ uma álgebra e $B \subset A$ uma subálgebra. Em teoria de representações, frequentemente se estuda a relação entre as categorias de módulos $A$-mod e $B$-mod. A pergunta mais interessante neste sentido é: Quando um B-módulo pode ser levantado num A-módulo? pois claramente, cada $A$-módulo é um $B$-módulo. Uma condição suficiente para que isso ocorra é que $A$ seja um $B$-módulo livre.

2. Sejam $A$ uma álgebra associativa e $\Gamma \subset A$ uma subálgebra comutativa. Em teoria de representações frequentemente se estudam módulos de Harish-Chandra com respeito a $\Gamma$, i.e., $A$-módulos $V$ que admitem uma decomposição como soma direta de $\Gamma$-módulos irredutíveis

$$
V=\bigoplus_{\lambda \in \Omega} V_{\lambda}
$$

em que $\Omega$ parametriza as classes de isomorfismos dos $\Gamma$-módulos irredutíveis. Um dos primeiros problemas no estudo dos módulos de Harish-Chandra é determinar quando um $\lambda$ dado pode ser levantado a um módulo de Harish-Chandra irredutível $V \operatorname{com} V_{\lambda} \neq 0$. O caso mais interessante é quando existe tal levantamento para qualquer $\lambda$. Isto é verdade quando $A$ é um $\Gamma$-módulo livre.

Neste sentido, no segundo capítulo deste trabalho são apresentados os conceitos básicos necessários para o desenvolvimento da tese, tais como, equidimensionalidade de variedades, interseção completa, sequências regulares e a relação entre os três conceitos. Essa relação permite explorar várias técnicas que serão usadas para obter os principais resultados desta tese.

No terceiro capítulo, começamos definindo a variedade de Gelfand-Tsetlin para $\mathfrak{g l}_{n}$, para depois apresentar o Teorema de Ovsienko. Provaremos a seguinte versão fraca do Teorema de Ovsienko (teorema (3.18)) com técnicas diferentes das apresentadas por S. Ovsienko em [Ovsienko (2003)]

\section{Versão fraca do Teorema de Ovsienko:}

A variedade algébrica

$$
V_{n}:=V\left(\sigma_{n 2}, \sigma_{n 3}, \sigma_{n 4}, \ldots, \sigma_{n n}\right) \subset \mathbb{A}_{k}^{\frac{(n+2)(n-1)}{2}}
$$

é equidimensional com dimensão

$$
\operatorname{dim}\left(V_{n}\right)=\frac{n(n-1)}{2}
$$

onde

$$
\sigma_{n j}=\sum_{n>t_{1}>t_{2}>\cdots>t_{j-1} \geq 1} X_{n t_{1}} X_{t_{1} t_{2}} \cdots X_{t_{j-2} t_{j-1}} X_{t_{j-1} n} ; j=2,3, \ldots, n
$$

B. Kostant e N. Wallach em [Kostant e Wallach (2006a)] e [Kostant e Wallach (2006b)] afirmam que todas as componentes regulares da variedade de Gelfand-Tsetlin são equidimensionais com dimensão $\frac{n(n-1)}{2}$. No terceiro capítulo, também mostraremos que esse fato é consequência da versão fraca e será apresentado no corolário (3.23) da seguinte forma:

\section{Corolário da versão fraca do Teorema de Ovsienko:}

Todas as componentes regulares da variedade de Gelfand-Tsetlin são isomorfas. Em particular, são isomorfas à componente irredutível

$$
V_{\leq}:=V\left(\left\{X_{i j}: 1 \leq i \leq j \leq n\right\}\right) .
$$


Existe uma função conhecida como "aplicação de Kostant-Wallach"[ver Kostant e Wallach (2006a)]

$$
\varphi: M_{n}(\mathbb{C}) \longrightarrow \mathbb{C}^{\frac{n(n+1)}{2}},
$$

cuja fibra no ponto zero $\varphi^{-1}(0)$ coincide com a variedade de Gelfand-Tsetlin para $\mathfrak{g l}_{n}$. Recentemente, M. Colarusso e S. Evens provaram em [Colarusso e Evens (2015)] que a fibra em zero $\varphi_{2}^{-1}(0)$ da função, chamada de aplicação parcial de Kostant-Wallach,

$$
\varphi_{2}: M_{n}(\mathbb{C}) \longrightarrow \mathbb{C}^{n-1} \times \mathbb{C}^{n}
$$

é equidimensional e nesse caso com dimensão $n^{2}-2 n+1$. Usando técnicas diferentes, no capítulo quatro, apresentamos uma generalização deste resultado. De forma precisa, definimos uma função que chamaremos de aplicação $k$-parcial de Kostant-Wallach

$$
\varphi_{k}: M_{n}(\mathbb{C}) \longrightarrow \mathbb{C}^{n-k+1} \times \cdots \times \mathbb{C}^{n-1} \times \mathbb{C}^{n},
$$

unificando as definições de aplicação de Kostant-Wallach e de aplicação parcial de Kostant-Wallach e provamos que a fibra em zero $\varphi_{k}^{-1}(0)$ é equidimensional com dimensão $n^{2}-(k+1) n+\frac{k(k+1)}{2}$. Além disso, provamos que se fixarmos $\alpha \in \mathbb{C}^{n-k+1} \times \cdots \times \mathbb{C}^{n-1} \times \mathbb{C}^{n}$, então a fibra $\varphi_{k}^{-1}(\alpha)$ é também equidimensional com dimensão $n^{2}-(k+1) n+\frac{k(k+1)}{2}$. Tudo isso é consequência do seguinte teorema (teorema (4.15)), o qual é o resultado principal do capítulo quatro:

\section{Teorema:}

Para todo $k=1,2, \ldots, n$ e todo $\beta \in \mathbb{C}^{n-k+1} \times \cdots \times \mathbb{C}^{n-1} \times \mathbb{C}^{n}$ a variedade

$$
\widetilde{V}_{\beta}^{k}=V\left(\left\{\overline{\gamma_{i j}}-\beta_{i j}: n-k+1 \leq i \leq n \text { e } 1 \leq j \leq i\right\}\right) \subset \mathbb{C}^{n^{2}}
$$

é equidimensional com dimensão

$$
\operatorname{dim}\left(\widetilde{V}_{\beta}^{k}\right)=n^{2}-n k+\frac{k(k-1)}{2},
$$

onde os $\overline{\gamma_{i j}}$ 's são os polinômios que determinam a variedade de Gelfand-Tsetlin para $\mathfrak{g l}_{n}$.

No quinto e último capítulo deste trabalho, apresentamos os Yangians restritos $Y_{p}\left(\mathfrak{g l}_{n}\right)$ de nível $p$ para $\mathfrak{g l}_{n}$ e sua variedade de Gelfand-Tsetlin $\mathfrak{G}$. Provamos a equidimensionalidade da variedade de Gelfand-Tsetlin $\mathfrak{G}$ para $Y_{2}\left(\mathfrak{g l}_{3}\right)$ na proposição (5.12), apresentando sua decomposição em componentes irredutíveis. Também apresentamos na proposição (5.13), uma decomposição (não em componentes irredutíveis) da variedade de Gelfand-Tsetlin para $Y_{p}\left(\mathfrak{g l}_{n}\right)$ da forma

$$
\mathfrak{G}=\mathfrak{G}_{1} \cup \mathfrak{G}_{2} \cup \cdots \cup \mathfrak{G}_{p+1} .
$$

No corolário (5.19), mostramos uma decomposição da subvariedade $\mathfrak{G}_{1}$ de $\mathfrak{G}$ para $Y_{p}\left(\mathfrak{g l}_{n}\right)$ na forma

$$
\begin{gathered}
\mathfrak{G}_{1}=\mathbb{W}_{1} \cup \mathbb{W}_{2} \text {, se } p=1,2 \\
\mathfrak{G}_{1}=\mathbb{W}^{p} \cup \mathbb{W}_{1} \cup \mathbb{W}_{2} \text {, se } p \geq 3 .
\end{gathered}
$$

Quando $n=3$, a variedade $\mathfrak{G}_{1}$ será chamada de versão fraca da variedade de Gelfand-Tsetlin $\mathfrak{G}$ para $Y_{p}\left(\mathfrak{g l}_{3}\right)$. Encerramos o quinto capítulo provando a equidimensionalidade de $\mathbb{W}^{p}, \mathbb{W}_{1}$ e $\mathbb{W}_{2}$ e obtendo, portanto, o resultado principal (teorema (5.25)) desse capítulo

\section{Teorema:}

Para todo $p$, a variedade $\mathfrak{G}_{1}$ para $Y_{p}\left(\mathfrak{g l}_{3}\right)$ é equidimensional com dimensão

$$
\operatorname{dim} \mathfrak{G}_{1}=3 p .
$$


Por fim, incluímos dois apêndices: $\mathrm{O}$ apêndice (A) contém alguns fatos que foram necessários para o capítulo 5, e no apêndice (B), estão todos os detalhes da decomposição em componentes irredutíveis da variedade de Gelfand-Tsetlin para $Y_{2}\left(\mathfrak{g l}_{3}\right)$. 


\section{Capítulo 2}

\section{Conceitos Preliminares}

Neste capítulo introduziremos conceitos preliminares e algumas propiedades que usaremos ao longo deste trabalho. Começaremos com alguns conceitos para poder definir e dar exemplos de álgebras de Lie, seguindo com a definição de álgebra envolvente universal e o Teorema de PoincaréBirkhoff-Witt. Seguidamente, apresentaremos algumas definições e fatos da Geometria Algébrica, tais como variedade algébrica, dimensão e equidimensionalidade. Continuaremos com conceitos de Álgebra Homológica, introduzindo o complexo de Koszul, sua homologia e o conceito de interseção completa. Finalmente, encerraremos este capítulo com definições e fatos da Álgebra Comutativa, tais como sequências regulares e a relação com equidimensionalidade e interseção completa.

Durante toda a tese, fixaremos $k$ como um corpo algébricamente fechado de caracterítica zero. Alguns dos resultados mencionados neste trabalho valem em um contexto mais geral, mas por simplicidade vamos nos restringir a este caso.

Definição 2.1. Sejam $V$ e $W$ dois $k$-espaços vetoriais.

1. Uma aplicação $n$-linear (ou aplicação multilinear) é uma aplicação

$$
B: \underbrace{V \times V \times \cdots \times V}_{n-\text { vezes }} \longrightarrow W,
$$

a qual é k-linear em cada coordenada.

2. Dizemos que a forma multilinear B é anti-simétrica se

$$
B\left(v_{1}, \ldots, v_{i}, \ldots, v_{j}, \ldots, v_{n}\right)=-B\left(v_{1}, \ldots, v_{j}, \ldots, v_{i}, \ldots, v_{n}\right) .
$$

3. Uma forma bilinear sobre $V$ é, simplesmente uma forma 2 -linear $B: V \times V \longrightarrow k$.

4. Dizemos que uma forma bilinear $B$ sobre $V$ é não-degenerada, se para todo $u \in V$ com $u \neq 0$, existe $v \in V$ tal que $B(u, v) \neq 0$.

Observação 2.2. $\operatorname{Sedim}_{k}(V)=n$, podemos identificar $V \operatorname{com} k^{n} \operatorname{como} k$-espaço vetorial. Seja $A \in M_{n}(k)$ uma matriz $n \times n$. Claramente a aplicação

$$
\begin{aligned}
B: V \times V & \longrightarrow k \\
(u, v) & \longrightarrow B(u, v):=u^{T} A v
\end{aligned}
$$

é uma forma bilinear. De fato, existe uma correspondência 1-1 entre as formas bilineares sobre $V$ e as matrizes quadradas $n \times n$ (a menos de semelhança de matrizes), da seguinte forma: 
Se B é uma forma bilinear sobre $V$ e $\left\{e_{1}, e_{2}, \ldots, e_{n}\right\}$ é uma base de $V$, então a matriz

$$
A=\left(\begin{array}{cccc}
B\left(e_{1}, e_{1}\right) & B\left(e_{1}, e_{2}\right) & \cdots & B\left(e_{1}, e_{n}\right) \\
B\left(e_{2}, e_{1}\right) & B\left(e_{2}, e_{2}\right) & \cdots & B\left(e_{2}, e_{n}\right) \\
\vdots & \vdots & \ddots & \vdots \\
B\left(e_{n}, e_{1}\right) & B\left(e_{n}, e_{2}\right) & \cdots & B\left(e_{n}, e_{n}\right)
\end{array}\right),
$$

chamada de matriz de $B$ relativa à base $\left\{e_{1}, e_{2}, \ldots, e_{n}\right\}$ satisfaz $B(u, v)=u^{T} A v, \forall u, v \in V . E$ caso $\left\{f_{1}, f_{2}, \ldots, f_{n}\right\}$ seja outra base de $V$ com matriz relativa $C$ à forma bilinear $B$, então

$$
C=P A P^{T},
$$

em que P é a matriz mudança de base.

Definição 2.3. Uma álgebra A é uma álgebra graduada, se existe uma coleção de subespaços vetoriais $A_{n}$ (chamada de graduação de A), tais que $A_{n} \cdot A_{m} \subseteq A_{n+m} e$

$$
A=\bigoplus_{n=0}^{\infty} A_{n}
$$

Definição 2.4. Sejam A e B duas álgebras graduadas com graduações $\left\{A_{n}\right\}_{n=0}^{\infty}$ e $\left\{B_{n}\right\}_{n=0}^{\infty}$ respectivamente. Um homomorfismo de álgebras graduadas $f: A \longrightarrow B$ é um homomorfismo de álgebras tal que

$$
f\left(A_{n}\right) \subseteq B_{n}
$$

\section{Exemplo 2.5.}

A álgebra de polinômios em $n$ variáveis $k\left[t_{1}, t_{2}, \ldots, t_{n}\right]$ é uma álgebra graduada, em que $A_{m}$ são os polinômios de grau $m$.

Definição 2.6. Uma álgebra A é uma álgebra filtrada se existe uma coleção de subespaços vetoriais $\left\{A_{n}\right\}_{n=0}^{\infty}$ (chamada de filtração de $A$ ), tais que
a. $A_{0} \subseteq A_{1} \subseteq A_{2} \subseteq \cdots$.
b. $A_{n} \cdot A_{m} \subseteq A_{n+m}$.
c. $A=\bigcup_{n=0}^{\infty} A_{n}$.

Observação 2.7. Se A é uma álgebra associativa filtrada cuja filtração é $\left\{A_{n}\right\}_{n=0}^{\infty}$

$$
A_{0} \subseteq A_{1} \subseteq A_{2} \subseteq \cdots,
$$

então:

1. Observemos que esta filtração induz uma álgebra graduada, que denotaremos por gr $(A)$ ou as vezes por simplicidade $\bar{A}$

$$
\operatorname{gr}(A)=\bigoplus_{n=0}^{\infty} \bar{A}_{n}
$$

onde

$$
\bar{A}_{n}:=A_{n} / A_{n-1}
$$

$e$

$$
A_{-1}=\{0\} .
$$

Esta graduação é chamada de graduação associada à filtração de A ou quando a filtração já está estabelecida é simplesmente chamada de graduação associada de A. 
2. Se B é uma subálgebra de A, então B também é uma álgebra filtrada, cuja filtração induzida é $\left\{B_{n}=A_{n} \cap B\right\}_{n=0}^{\infty}$

$$
B_{0} \subseteq B_{1} \subseteq B_{2} \subseteq \cdots,
$$

portanto, do item anterior temos a álgebra graduada gr $(B)$ e a inclusão $i: B \hookrightarrow A$, também induz um morfismo de álgebras graduadas

$$
\operatorname{gr}(i): \begin{array}{cc}
\operatorname{gr}(B) & \longrightarrow \operatorname{gr}(A) \\
v_{n}+B_{n-1} & \longmapsto \operatorname{gr}(i)\left(v_{n}+B_{n-1}\right):=v_{n}+A_{n-1},
\end{array}
$$

o qual é injetor, pois se $v_{n}+B_{n-1}, w_{n}+B_{n-1} \in B_{n} / B_{n-1}$ são tais que

$$
\operatorname{gr}(i)\left(v_{n}+B_{n-1}\right)=\operatorname{gr}(i)\left(w_{n}+B_{n-1}\right)
$$

então $v_{n}+A_{n-1}=w_{n}+A_{n-1}$. Isto implica que $v_{n}-w_{n} \in A_{n-1}$, assim $v_{n}-w_{n} \in A_{n-1} \cap B$ e, como $A_{n-1} \cap B=B_{n-1}$, concluimos que

$$
v_{n}+B_{n-1}=w_{n}+B_{n-1} .
$$

Portanto, podemos identificar gr (B) como a subálgebra de gr $(A)$ cujos monômios $v_{n}$ estão em $B_{n}$.

\section{1 Álgebras de Lie}

Agora definiremos os conceitos de álgebras de Lie, subálgebras de Lie e daremos alguns exemplos que serão importantes ao longo do trabalho.

Definição 2.8. Uma álgebra de Lie é um espaço vetorial $\mathfrak{g}$ munido de um produto

$$
[,]: \mathfrak{g} \times \mathfrak{g} \longrightarrow \mathfrak{g}
$$

chamado de colchete de Lie com as seguintes propriedades:

a. Ébilinear.

b. É Antisimétrico (equivalentemente, $[x, x]=0$ para todo $x \in \mathfrak{g}$ ).

c. Satisfaz a Identidade de Jacobi, i.e., para todo $x, y, z \in \mathfrak{g}$

$$
[x,[y, z]]+[y,[z, x]]+[z,[x, y]]=0 .
$$

Definição 2.9. Uma álgebra de Lie g é dita abeliana se

$$
[x, y]=0, \forall x, y \in \mathfrak{g} .
$$

\section{Exemplo 2.10.}

a. Para uma álgebra associativa $A$, denotemos por $A^{-}$a álgebra de Lie associada a $A$ com colchete de Lie dado pelo comutador, i.e.,

$$
[x, y]:=x y-y x, \forall x, y \in A .
$$

b. Sejam $V$ um espaço vetorial e $\operatorname{End}(V)$ o conjunto de todas as transformações lineares $V \longrightarrow V$ com estrutura de álgebra associativa. Denotemos a álgebra $\operatorname{End}(V)$ vista como álgebra de Lie por $\mathfrak{g l}(V)$, i.e.,

$$
\mathfrak{g l}(V):=\operatorname{End}(V)^{-}
$$

a qual é chamada de Álgebra Linear Geral. 
Observemos que se $\operatorname{dim}_{k}(V)=n$, então podemos identificar $\operatorname{End}(V)$ com o espaço vetorial $M_{n}(k)$ das matrices quadradas $n \times n \mathrm{e}$

$$
\mathfrak{g l}_{n}:=\mathfrak{g l}(n, k):=\mathfrak{g l}_{n}(k):=\mathfrak{g l}(V)=M_{n}(k)^{-},
$$

conhecida como Álgebra de Lie linear.

Definição 2.11. Uma subálgebra de uma álgebra de Lie gé um subespaço vetorial $\mathfrak{h}$ de $\mathfrak{g}$ tal que

$$
[x, y] \in \mathfrak{h}, \forall x, y \in \mathfrak{h} .
$$

\section{Exemplo 2.12.}

a. Álgebra Linear Especial

$$
\mathfrak{s l}(n, k):=\left\{X \in \mathfrak{g l}_{n}(k): \operatorname{tr}(X)=0\right\},
$$

onde $\operatorname{tr}(X)$ denota o traço da matriz $X$. Também é denotada por $\mathfrak{s l}(n, k):=\mathfrak{s l}_{n}(k):=\mathfrak{s l}_{n}:=\mathfrak{s l}(n)$.

b. Álgebra Simplética: Sejam $V$ um espaço vetorial $\operatorname{com}_{\operatorname{dim}}(V)=2 n$ e $\left\{v_{1}, v_{2}, \ldots, v_{2 n}\right\}$ uma base. Definamos como na observação (2.2) a forma bilinear $B$, anti-simétrica e não-degenerada sobre $V$ dada pela matriz

$$
J=\left(\begin{array}{cc}
0 & I_{n} \\
-I_{n} & 0
\end{array}\right)
$$

onde $I_{n}$ é a matriz identidade $n \times n$. Portanto a álgebra simplética é definida como

$$
\mathfrak{s p}(V):=\{T \in \operatorname{End}(V): B(T(u), v)=-B(u, T(v))\} .
$$

Em termos de matrizes temos que

$$
\mathfrak{s p}(V)=\left\{X \in \mathfrak{g l}_{2 n}(k): X J+J X^{T}=0\right\} .
$$

É comum ver na literátura as notações $\mathfrak{s p}(V):=\mathfrak{s p}(2 n, k):=\mathfrak{s p}_{2 n}(k):=\mathfrak{s p}_{2 n}:=\mathfrak{s p}(2 n)$.

Definição 2.13. Sejam $\left(\mathfrak{g},[,]_{1}\right) e\left(\mathfrak{h},[,]_{2}\right)$ duas álgebras de Lie. Dizemos que uma transformação linear $f: \mathfrak{g} \longrightarrow \mathfrak{h}$ é um homomorfismo de álgebras de Lie se satisfaz

$$
f\left([x, y]_{1}\right)=[f(x), f(y)]_{2}, \forall x, y \in \mathfrak{g} .
$$

\section{2 Álgebra Envolvente Universal}

Nesta seção introduziremos a álgebra tensorial de um módulo, a qual permitirá definir as álgebras simétrica e exterior de um módulo e a álgebra envolvente universal de uma álgebra de Lie. Encerraremos esta seção com o necessário para enunciar o Teorema de Poincaré-Birkhoff-Witt. Para mais detalhes sobre a álgebra tensorial, simétrica e exterior ver [Bourbaki (1989)] nas páginas 484-522 e sobre a álgebra envolvente universal e o Teorema de Poincaré-Birkhoff-Witt ver [Humphreys (1978)] nas páginas $90-94$.

\subsection{1 Álgebras Tensorial, Simétrica e Exterior}

Sejam $R$ um anel comutativo, $M$ um $R$-módulo e consideremos os seguintes $R$-módulos:

$$
\begin{aligned}
& T^{0} M=R, \\
& T^{1} M=M, \\
& T^{2} M=M \otimes_{R} M,
\end{aligned}
$$




$$
T^{n} M=\underbrace{M \otimes_{R} M \otimes_{R} \cdots \otimes_{R} M}_{n \text { vezes }},
$$

e o $R$-módulo

$$
T(M)=\bigoplus_{n=0}^{\infty} T^{n} M,
$$

no qual podemos introduzir um produto associativo, definido sobre os geradores homogêneos de $T(M)$, da seguinte forma

$$
\underbrace{\left(v_{1} \otimes v_{2} \otimes \cdots \otimes v_{n}\right)}_{\in T^{n} M} \underbrace{\left(w_{1} \otimes w_{2} \otimes \cdots \otimes w_{m}\right)}_{\in T^{m} M}=\underbrace{v_{1} \otimes v_{2} \otimes \cdots \otimes v_{n} \otimes w_{1} \otimes w_{2} \otimes \cdots \otimes w_{m}}_{\in T^{n+m} M},
$$

pois todo elemento de $T(M)$ é uma soma finita $\sum z_{n}$, com $z_{n} \in T^{n} M$ e como $z_{n}$ é uma combinação linear de monômios, podemos estender por $R$-bilinearidade o produto a todo o $R$-módulo $T(M)$. Desta forma, $T(M)$ é uma álgebra associativa graduada, pois

$$
T^{n} M \cdot T^{m} M=T^{n+m} M, \forall n, m .
$$

Esta álgebra é chamada de álgebra tensorial de $M$.

Proposição 2.14 (Propriedade universal da álgebra tensorial).

Sejam M um R-módulo e A uma R-álgebra associativa com unidade 1. Para qualquer homomorfismo de $R$-módulos $\phi: M \longrightarrow A$, existe um único homomorfismo de $R$-álgebras $\Phi: T(M) \longrightarrow A$ tal que, $\Phi(1)=1 e \Phi \circ i=\phi$, onde $i: M \longrightarrow T(M)$ é a inclusão, i.e. o seguinte diagrama comuta

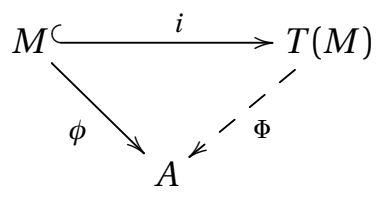

Demonstração: Basta considerar

$$
\Phi\left(v_{1} \otimes v_{2} \otimes \cdots \otimes v_{n}\right):=\phi\left(v_{1}\right) \phi\left(v_{2}\right) \ldots \phi\left(v_{n}\right) .
$$

Proposição 2.15. Sejam $M$ um $R$-módulo livre e $\left\{e_{1}, e_{2}, \ldots, e_{n}\right\}$ uma base de $M$, então:

1. Para qualquer $m \geq 1, T^{m} M$ é um $R$-módulo livre com posto $n^{m}$ e os tensores da forma

$$
e_{i_{1}} \otimes e_{i_{2}} \otimes \cdots e_{i_{m}}
$$

formam uma base para $T^{m} M$.

2. A álgebra tensorial $T(M)$ é isomorfa à álgebra de polinômios $R\left\langle x_{1}, x_{2}, \ldots, x_{n}\right\rangle$ nas variáveis não comutativas $x_{1}, x_{2}, \ldots, x_{n}$ com coeficientes em $R$.

Definição 2.16. Sejam $M$ um R-módulo, $T(M)$ sua álgebra tensorial e $C(M)$ o ideal bilateral de $T(M)$ gerado por todos os elementos da forma

$$
x \otimes y-y \otimes x, \forall x, y \in M
$$

A álgebra quociente

$$
S(M):=T(M) / C(M)
$$


é dita álgebra simétrica de $M$. Denotemos por $\sigma$ a projeção canônica de R-álgebras

$$
\begin{aligned}
\sigma: T(M) & \longrightarrow S(M) \\
x & \longmapsto \sigma(x):=\bar{x} .
\end{aligned}
$$

\section{Observação 2.17.}

1. Para cada $n \geq 1$, seja $S^{n} M:=\sigma\left(T^{n} M\right)$ a imagem de $T^{n} M$ em $S(M)$. Portanto, $S(M)$ é uma álgebra graduada com graduação

$$
S(M)=\bigoplus_{n=0}^{\infty} S^{n} M
$$

2. $S(M)$ é uma R-álgebra comutativa.

Proposição 2.18. Sejam $M$ um $R$-módulo livre e $\left\{e_{1}, e_{2}, \ldots, e_{n}\right\}$ uma base de $M$, então $S(M)$ é isomorfa $a R\left[x_{1}, x_{2}, \ldots, x_{n}\right]$ (álgebra de polinômios com coeficientes em $R$ ) como uma $R$-álgebra graduada.

Definição 2.19. Sejam $M$ um R-módulo, $T(M)$ sua álgebra tensorial e $A(M)$ o ideal bilateral de $T(M)$ gerado por todos os elementos da forma

$$
x \otimes x, \forall x \in M .
$$

Aálgebra quociente

$$
\bigwedge M:=T(M) / A(M)
$$

é chamada de álgebra exterior de $M$.

O produto em $\bigwedge M$ é denotado pelo símbolo $\wedge$, i.e., para $m_{1}, m_{2}, \ldots, m_{n} \in M$ denotamos por

$$
m_{1} \wedge m_{2} \wedge \cdots \wedge m_{n}
$$

a classe de

$$
m_{1} \otimes m_{2} \otimes \cdots \otimes m_{n}
$$

em $\bigwedge(M)$.

Similar à álgebra simétrica, a álgebra exterior de $M$ tem uma graduação natural

$$
\bigwedge M=\bigoplus_{n=o}^{\infty} \bigwedge^{n} M
$$

em que $\bigwedge^{n} M$ é o quociente de $T^{n} M$ em $\bigwedge M$, isto é,

$$
\bigwedge^{n} M=T^{n} M / A_{n}(M)
$$

com

$$
A_{n}(M):=\left\langle v_{1} \otimes v_{2} \otimes \cdots \otimes v_{n} \in T^{n} M: \text { existem } i \neq j \text { tais que } v_{i}=v_{j}\right\rangle \subset A(M) .
$$

$\bigwedge^{n} M$ é chamada de $n$-ésima potência exterior de $M$.

Proposição 2.20 (Propriedade universal da $n$-ésima potência exterior de $M$ ).

Seja $n \in \mathbb{N}$. Se existir um $R$-módulo $N$ e uma aplicação $n$-linear alternada $f: M^{n} \longrightarrow N$ (isto é, $f\left(x_{1}, x_{2}, \ldots, x, \ldots, x, \ldots, x_{n}\right)=0$ ), então existe um único homomorfismo de $R$-módulos $g: \bigwedge^{n} M \longrightarrow N$, tal que $g \circ \wedge_{n}=f$, ou seja, o seguinte diagrama comuta

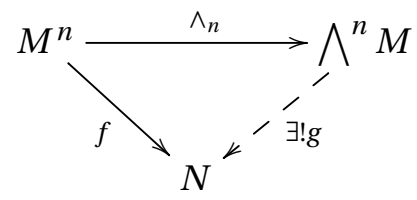


em que, $\wedge_{n}$ é a aplicação n-linear e anti-simétrica

$$
\wedge_{n}: \begin{array}{cl}
M^{n} & \longrightarrow \bigwedge^{n} M \\
\left(m_{1}, m_{2}, \ldots, m_{n}\right) & \longmapsto \wedge_{n}\left(m_{1}, m_{2}, \ldots, m_{n}\right):=m_{1} \wedge m_{2} \wedge \ldots \wedge m_{n} .
\end{array}
$$

Proposição 2.21. Seja $M$ um R-módulo livre, de posto $n$ e com base $\left\{e_{1}, e_{2}, \ldots, e_{n}\right\}$, então:

1. $\bigwedge M$ é isomorfa a $R\left\langle x_{1}, x_{2}, \ldots, x_{n}\right\rangle /$ I como $R$-álgebra, em que I é o ideal bilateral gerado pelo conjunto

$$
\left\{x_{i}^{2}, x_{i} x_{j}+x_{j} x_{i}: i, j=1,2, \ldots, n\right\} .
$$

Portanto,

$$
\bigwedge M \cong\left\langle e_{1}, e_{2}, \ldots, e_{n}: e_{i} \wedge e_{i}=0, e_{i} \wedge e_{j}=-e_{j} \wedge e_{i}\right\rangle .
$$

2. Para cada $k \geq 1, \bigwedge^{k} M$ é um $R$-módulo livre com base

$$
\left\{e_{i_{1}} \wedge e_{i_{2}} \wedge \ldots \wedge e_{i_{k}}: 1 \leq i_{1}<i_{2}<\cdots<i_{k} \leq n\right\}
$$

Em particular, seu posto é( $\left.\begin{array}{l}n \\ k\end{array}\right)$.

3. $\bigwedge M$ é um R-módulo livre finitamente gerado de posto $2^{n}$.

\subsection{2 Álgebra Envolvente Universal}

Nesta seção seguiremos [Humphreys (1978)], páginas 90-94.

Definição 2.22. Seja $\mathfrak{g}$ uma álgebra de Lie sobre $k$. Uma álgebra envolvente universal de $\mathfrak{g}$ é um par $(U, i)$, em que $U$ é uma $k$-álgebra associativa com 1 e $i: \mathfrak{g} \longrightarrow U^{-}$um homomorfismo de álgebras de Lie, tais que $(U, i)$ satisfaz a seguinte propriedade universal:

Para qualquer $k$-álgebra associativa A com 1 e qualquer homomorfismo de álgebras de Lie

$$
\phi: \mathfrak{g} \longrightarrow A^{-}
$$

i.e., $\phi([x, y])=\phi(x) \phi(y)-\phi(y) \phi(x)$ para todo $x, y \in \mathfrak{g}$, existe um único $\Psi: U \longrightarrow A$ homomorfismo de álgebras associativas, tal que

$$
\Psi\left(1_{U}\right)=1_{A} \text { e } \Psi \circ i=\phi,
$$

i.e. o seguinte diagrama comuta

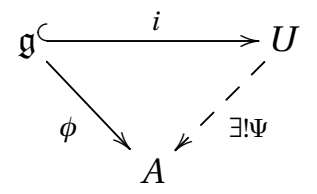

Proposição 2.23 (Unicidade).

Sejam $\left(U_{1}, i_{1}\right)$ e $\left(U_{2}, i_{2}\right)$ duas álgebras envolventes universais de uma álgebra de Lie $\mathfrak{g}$. Então, existe um único isomorfismo $j: U_{1} \longrightarrow U_{2}$ de álgebras associativas tal que $i_{2}=j i_{1}$.

Demonstração: Segue-se da propriedade universal da álgebra envolvente universal.

\section{Proposição 2.24 (Existência).}

Seja $\mathfrak{g}$ uma $k$-álgebra de Lie, então existe uma única (a menos de isomorfismo) álgebra envolvente universal de $\mathfrak{g}$.

Demonstração: Consideremos a álgebra tensorial $T(\mathfrak{g})$ de $\mathfrak{g}$ e o ideal bilateral $J$ de $T(\mathfrak{g})$ gerado por todos os elementos da forma

$$
x \otimes y-y \otimes x-[x, y], \forall x, y \in \mathfrak{g} .
$$


Agora, definamos a álgebra associativa $U(\mathfrak{g}):=T(\mathfrak{g}) / J$ e a aplicação

$$
\begin{aligned}
i: \mathfrak{g} & \longrightarrow U(\mathfrak{g})^{-} \\
x & \longrightarrow i(x):=x+J,
\end{aligned}
$$

a qual claramente é $k$-linear, além disso é um homomorfismo de álgebras de Lie, pois

$$
i([x, y])=[x, y]+J=x \otimes y-y \otimes x+J, \forall x, y \in \mathfrak{g} .
$$

Para provar que vale a propriedade universal, consideremos uma $k$-álgebra associativa $A$ com 1 e um homorfismo de álgebras de Lie $\phi: \mathfrak{g} \longrightarrow A^{-}$. Pela propriedade universal da álgebra tensorial, existe um único homomorfismo de $k$-álgebras $\Phi: T(\mathfrak{g}) \longrightarrow A$, tal que $\Phi \circ j=\phi$, onde $j: \mathfrak{g} \longrightarrow T(\mathfrak{g})$ é a inclusão.

Observemos que $J \subset \operatorname{ker} \Phi$, pois como $\Phi$ é homomorfismo de álgebras

$$
\begin{aligned}
\Phi(x \otimes y-y \otimes x-[x, y]) & =\Phi(x) \Phi(y)-\Phi(y) \Phi(x)-\Phi([x, y]) \\
& =\phi(x) \phi(y)-\phi(y) \phi(x)-\phi([x, y]) \\
& =\phi(x \otimes y-y \otimes x-[x, y])
\end{aligned}
$$

e como $\phi$ é um homomorfismo de álgebras de Lie temos

$$
\Phi(x \otimes y-y \otimes x-[x, y])=0 .
$$

Agora, do fato que $J \subset \operatorname{ker} \Phi$, segue que existe um homomorfismo de $k$-álgebras $\Psi: U(\mathfrak{g}) \longrightarrow A$, tal que $\Psi(x+J)=\Phi(x), \forall x \in U(\mathfrak{g})$. Além disso, temos $\Psi(1)=1 \mathrm{e} \Psi i(x)=\phi(x), \forall x \in \mathfrak{g}$.

Para provar a unicidade (na propriedade universal da álgebra envolvente universal), consideremos outro homomorfismo de $k$-álgebras $\Psi^{\prime}: U(\mathfrak{g}) \longrightarrow A$, tal que $\Psi^{\prime}(1)=1$ e $\Psi^{\prime} i(x)=\phi(x), \forall x \in \mathfrak{g}$. Seja $w_{1} \otimes w_{2} \otimes \cdots \otimes w_{n}+J \in U(\mathfrak{g})$ arbitrário, logo

$$
\begin{aligned}
\Psi^{\prime}\left(w_{1} \otimes w_{2} \otimes \cdots \otimes w_{n}+J\right) & =\Psi^{\prime}\left(\left(w_{1}+J\right)\left(w_{2}+J\right) \cdots\left(w_{n}+J\right)\right) \\
& =\Psi^{\prime}\left(w_{1}+J\right) \Psi^{\prime}\left(w_{2}+J\right) \cdots \Psi^{\prime}\left(w_{n}+J\right) \\
& =\phi\left(w_{1}\right) \phi\left(w_{2}\right) \cdots \phi\left(w_{n}\right) \\
& =\Phi\left(w_{1}\right) \Phi\left(w_{2}\right) \cdots \Phi\left(w_{n}\right) \\
& =\Psi\left(w_{1} \otimes w_{2} \otimes \cdots \otimes w_{n}+J\right) .
\end{aligned}
$$

\section{Exemplo 2.25.}

Seja $\mathfrak{g}$ uma álgebra de Lie abeliana, então $J$ seria o ideal bilateral formado por todos os elementos da forma

$$
x \otimes y-y \otimes x, \forall x, y \in \mathfrak{g} .
$$

Portanto $U(\mathfrak{g}) \cong S(\mathfrak{g})$ como $k$-álgebras.

\subsubsection{Teorema de Poincaré-Birkhoff-Witt}

Em [Grivel (2004)] e [Shepler e Witherspoon (2014)], podem ser encontrados detalhes sobre a historia do Teorema de Poincaré-Birkhoff-Witt.

Notação 2.26. Para facilitar a leitura, usaremos as seguintes notações:

$$
\begin{aligned}
T & =T(\mathfrak{g}) \\
S & =S(\mathfrak{g}) \\
U & =U(\mathfrak{g}) \\
T^{n} & =T^{n} \mathfrak{g}
\end{aligned}
$$




$$
S^{n}=S^{n} \mathfrak{g}
$$

Consideremos uma filtração sobre $T$ e sobre $U$ dada por:

$$
\begin{aligned}
T_{m} & :=T^{0} \oplus T^{1} \oplus T^{2} \oplus \cdots \oplus T^{m} \\
U_{m} & :=\pi\left(T_{m}\right) \\
U_{-1} & :=\{0\}
\end{aligned}
$$

onde $\pi: T(\mathfrak{g}) \longrightarrow U(\mathfrak{g})$ é a projeção canônica. Logo

$$
\begin{array}{r}
U_{m} U_{p} \subset U_{m+p} \\
U_{m} \subset U_{m+1} .
\end{array}
$$

Também consideremos o $k$-espaço vetorial

$$
G^{m}:=U_{m} / U_{m-1} .
$$

A multiplicação em $U$ define uma aplicação $k$-bilinear

$$
\begin{aligned}
G^{m} \times G^{p} & \longrightarrow G^{m+p} \\
(\bar{u}, \bar{v}) & \longmapsto \overline{u v}
\end{aligned}
$$

Claramente, isso pode se estender para uma aplicação bilinear

$$
\operatorname{gr}(U) \times \operatorname{gr}(U) \longrightarrow \operatorname{gr}(U),
$$

em que

$$
\operatorname{gr}(U)=\bigoplus_{n=0}^{\infty} G^{m},
$$

a qual é a álgebra graduada associativa com 1 associada à álgebra $U=U(\mathfrak{g})$.

Agora reparemos que a restrição de $\pi$ a $T^{m}$ é uma transformação $k$-linear

$$
\left.\pi\right|_{T^{m}}: T^{m} \longrightarrow \pi\left(T^{m}\right) \subset \pi\left(T_{m}\right)=U_{m}
$$

logo

$$
\begin{aligned}
\phi_{m}: T^{m} & \longrightarrow G^{m} \\
w & \longrightarrow \phi_{m}(w):=\pi(w)+U_{m-1}
\end{aligned}
$$

é uma transformação $k$-linear sobrejetora. Portanto, estendendo por $k$-linearidade, temos o homomorfismo de $k$-álgebras $\phi: T \longrightarrow \operatorname{gr}(U)$ sobrejetor, $\operatorname{com} \phi(1)=1 \mathrm{e}$

$$
\phi\left(v_{1} \otimes v_{2} \otimes \cdots \otimes v_{m}\right)=\phi_{m}\left(v_{1} \otimes v_{2} \otimes \cdots \otimes v_{m}\right),
$$

i.e., $\left.\phi\right|_{T^{m}}=\phi_{m}$ e portanto $\phi$ é sobrejetora.

Lema 2.27. $\phi: T \longrightarrow \operatorname{gr}(U)$ é um homomorfismo de álgebras. Além disso, $\phi(C(\mathfrak{g}))=0$ e assim $\phi$ induz um homomorfismo de álgebras $\omega: S=T / C(\mathfrak{g}) \longrightarrow \operatorname{gr}(U)$.

Demonstração: Basta provar que $\phi(x \otimes y-y \otimes x)=0, \forall x, y \in \mathfrak{g}$. De fato, note que $x \otimes y-y \otimes x \in T^{2} \subset T_{2}$, $\log \pi(x \otimes y-y \otimes x) \in U_{2}$, mas

$$
(x \otimes y-y \otimes x)+J=[x, y]+J \text { em } U,
$$

logo em $U$

$$
\pi(x \otimes y-y \otimes x)=\pi([x, y]) \in U_{1}
$$


assim

$$
\phi(x \otimes y-y \otimes x)=\phi_{2}(x \otimes y-y \otimes x)=\pi(x \otimes y-y \otimes x)+U_{1}=\pi([x, y])+U_{1}=0 .
$$

Portanto, temos o homomorfismo de $k$-álgebras sobrejetor

$$
\begin{array}{ccc}
\omega: \quad \begin{array}{l}
S \\
v+C(\mathfrak{g})
\end{array} & \longrightarrow \omega(v+C(\mathfrak{g})):=\phi(v) .
\end{array}
$$

Além disso, notemos que

$$
\begin{array}{ccc}
\left.\omega\right|_{S^{m}}: \quad \begin{array}{c}
S^{m} \\
v+C(\mathfrak{g})
\end{array} & \longrightarrow G^{m} \\
& \longrightarrow(v+C(\mathfrak{g})):=\phi(v)=\phi_{m}(v)=\pi(v)+U_{m-1},
\end{array}
$$

também é sobrejetor e $k$-linear.

O seguinte teorema é o resultado principal desta seção sobre $U(\mathfrak{g})$, conhecido como Teorema de Poincaré-Birkhoff-Witt (ou Teorema PBW).

Teorema 2.28 (Teorema de Poincaré-Birkhoff-Witt (PBW)).

O homomorfismo $\omega: S=T / C(\mathfrak{g}) \longrightarrow \operatorname{gr}(U)$ é um isomorfismo de álgebras.

\subsection{Geometria Algébrica}

Como dito anteriormente, durante toda a tese fixaremos $k$ como um corpo algébricamente fechado com característica zero, por exemplo, o corpo dos números complexos $k=\mathbb{C}$.

Nesta seção apresentaremos os conceitos e propriedades de variedades algébricas, ideal associado a uma variedade, componentes irredutíveis, dimensão de uma variedade e de um anel. Para esta seção, todos os anéis e as álgebras são comutativos.

Para começar fixemos o espaço onde queremos trabalhar.

Definição 2.29. Definamos por $\mathbb{A}_{k}^{n}=k^{n}$ (ou simplesmente $\mathbb{A}^{n}$ ou $k^{n}$ ) o espaço afim. Aquí esqueceremos a estrutura de $k^{n}$ como espaço vetorial.

Definição 2.30. Seja $S \subset k\left[x_{1}, x_{2}, \ldots, x_{n}\right]$. Definamos o conjunto de zeros de $S$ como

$$
V(S):=\left\{\left(a_{1}, a_{2}, \ldots, a_{n}\right) \in \mathbb{A}^{n}: f\left(a_{1}, a_{2}, \ldots, a_{n}\right)=0, \forall f \in S\right\} .
$$

Este conjunto é chamado de variedade algébrica afim associada ao conjunto $S$ (ou simplesmente variedade algébrica).

Dizemos que $X \subset \mathbb{A}^{n}$, é um conjunto algébrico (ou variedade algébrica) se $X=V(S)$ para algum $S \subset k\left[x_{1}, x_{2}, \ldots, x_{n}\right]$.

Observação 2.31. Sejam $S \subset k\left[x_{1}, x_{2}, \ldots, x_{n}\right]$ e $I=\langle S\rangle$ o ideal de $k\left[x_{1}, x_{2}, \ldots, x_{n}\right]$ gerado por $S$. Não é dificil ver que

$$
V(I)=V(S)
$$

Teorema 2.32 (Teorema da base de Hilbert).

Sejam $k$ um corpo e I um ideal de $k\left[x_{1}, x_{2}, \ldots, x_{n}\right]$, então I é finitamente gerado, ou seja, existe $m \geq 0$ e $f_{1}, f_{2}, \ldots, f_{m} \in I$ tais que

$$
I=\left\langle f_{1}, f_{2}, \ldots, f_{m}\right\rangle .
$$

Demonstração: Ver [Atiyah e Macdonald (1969)] na página 81.

Observação 2.33. Seja $S \subset k\left[x_{1}, x_{2}, \ldots, x_{n}\right]$, da observação anterior e o Teorema da base de Hilbert tem-se

$$
V(S)=V(I)=V\left(\left\langle f_{1}, f_{2}, \ldots, f_{m}\right\rangle\right)=V\left(f_{1}, f_{2}, \ldots, f_{m}\right)
$$

para alguns $f_{1}, f_{2}, \ldots, f_{m} \in I=\langle S\rangle$. 
Definição 2.34. Seja I um ideal de um anel R. Definimos o radical de I como

$$
\sqrt{I}:=\left\{x \in R: x^{n} \in I, \text { para algum } n \in \mathbb{N}\right\} .
$$

Dizemos, que I é um ideal radical se $I=\sqrt{I}$.

\section{Teorema 2.35 (Teorema dos zeros de Hilbert).}

Sejam $k$ um corpo algébricamente fechado e I um ideal de $k\left[x_{1}, x_{2}, \ldots, x_{n}\right]$, então

$$
\sqrt{I}:=\left\{f \in k\left[x_{1}, x_{2}, \ldots, x_{n}\right]: f(x)=0, \forall x \in V(I)\right\} .
$$

Demonstração: Ver o teorema 25 de [Matsumura (1970)] nas páginas 93 - 94 ou o teorema 14 de [Zariski e Samuel (1975)] na página 164.

Definição 2.36. Seja $X \subset \mathbb{A}_{k}^{n}$, definimos o ideal associado a $X$ como

$$
I(X):=\left\{f \in k\left[x_{1}, x_{2}, \ldots, x_{n}\right]: f(x)=0, \forall x \in X\right\} .
$$

\section{Observação 2.37.}

1. I(X) é um ideal radical de $k\left[x_{1}, x_{2}, \ldots, x_{n}\right]$.

2. Temos as correspondências biunívocas

$$
\begin{aligned}
& V: \quad\left\{\text { Ideais radicais de } k\left[x_{1}, x_{2}, \ldots, x_{n}\right]\right\} \quad \longrightarrow \quad\left\{\text { Variedades Algébricas em } \mathbb{A}^{n}\right\} \\
& I=\left\langle f_{1}, f_{2}, \ldots, f_{n}\right\rangle \quad \longmapsto V(I),
\end{aligned}
$$

$I: \quad\left\{\right.$ Variedades Algébricas em $\left.\mathbb{A}^{n}\right\} \longrightarrow$ IIdeais radicais de $\left.k\left[x_{1}, x_{2}, \ldots, x_{n}\right]\right\}$

$$
X \quad \longmapsto I(X) \text {. }
$$

Uma é a inversa da outra e tais correspondências invertem a inclusão.

Ver corolários 1 e 2 de [Zariski e Samuel (1975)] na página 167.

Definição 2.38. Uma variedade algébrica $X$ de $\mathbb{A}_{k}^{n}$ é dita irredutível se não existem $X_{1}, X_{2}$ variedades algébricas não vazias tais que

$$
X=X_{1} \cup X_{2} \text {. }
$$

Proposição 2.39. Seja $X$ uma variedade algébrica de $\mathbb{A}_{k}^{n}$, então

$$
X \text { é irredutível } \Longleftrightarrow I(X) \text { é um ideal primo de } k\left[x_{1}, x_{2}, \ldots, x_{n}\right] \text {. }
$$

Demonstração: Ver o teorema 12 de [Zariski e Samuel (1975)] na página 162.

\section{Proposição 2.40.}

$$
\begin{gathered}
\boldsymbol{a}=\left(a_{1}, a_{2}, \ldots, a_{n}\right) \in \mathbb{A}_{k}^{n} \Longrightarrow \boldsymbol{m}_{\boldsymbol{a}}=I(\{a\}) \text { é um ideal maximal de } k\left[x_{1}, x_{2}, \ldots, x_{n}\right] . \\
V(\boldsymbol{m})=\{\boldsymbol{a}\}, \text { para algum } \boldsymbol{a} \in \mathbb{A}_{k}^{n} \Longleftarrow \boldsymbol{m} \text { é um ideal maximal de } k\left[x_{1}, x_{2}, \ldots, x_{n}\right] .
\end{gathered}
$$

Demonstração: Ver corolário 2 de [Matsumura (1970)] na página 91. 
Observação 2.41. Temos as seguintes correspondências biunívocas induzidas por $V$ e I:

$$
\begin{aligned}
& \{\text { Conjuntos algébricos }\} \underset{V}{\gtrless}\left\{\text { Ideais radicais de } k\left[x_{1}, x_{2}, \ldots, x_{n}\right]\right\} \\
& \{\text { Conjuntos algébricos irredutíveis }\} \underset{V}{\stackrel{I}{\rightleftarrows}}\left\{\text { Ideais primos de } k\left[x_{1}, x_{2}, \ldots, x_{n}\right]\right\} \\
& \mathbb{A}_{k}^{n}=\left\{\text { Pontos de } \mathbb{A}_{k}^{n}\right\} \underset{I}{\stackrel{V}{\rightleftarrows}}\left\{\text { Ideais maximais de } k\left[x_{1}, x_{2}, \ldots, x_{n}\right]\right\}
\end{aligned}
$$

Definição 2.42. Seja $X \subset \mathbb{A}_{k}^{n}$ um conjunto algébrico. Definimos o anel de coordenadas de $X$ como

$$
A(X):=k\left[x_{1}, x_{2}, \ldots, x_{n}\right] / I(X) .
$$

\section{Observação 2.43.}

1. Todo anel de coodenadas de $A(X)$ é uma $k$-álgebra finitamente gerada sem elementos nilpotentes ( $a \in R$ é nilpotente em $R$ se existe $n \in \mathbb{N}$ tal que $a^{n}=0$ ).

2. Toda $k$-álgebra finitamente gerada sem elementos nilpotentes é o anel de coordenadas de algum conjunto algébrico.

Definição 2.44. Uma $k$-álgebra associativa, comutativa e finitamente gerada é chamada de $k$-álgebra afim. Uma $k$-álgebra afim é chamada de reduzida se não tem elementos nilpotentes.

Observação 2.45. Seja $X \subset \mathbb{A}_{k}^{n}$ um conjunto algébrico. A projeção natural

$$
\pi: k\left[x_{1}, x_{2}, \ldots, x_{n}\right] \longrightarrow A(X)=k\left[x_{1}, x_{2}, \ldots, x_{n}\right] / I(X),
$$

induz a correspondência biunívoca

$$
\left.\left\{\text { Ideais de } k\left[x_{1}, x_{2}, \ldots, x_{n}\right] \text { que contem } I(X)\right\} \longleftrightarrow{ }^{1-1} \longrightarrow \text { Ideais de } A(X)\right\}
$$

esta correspondência preserva inclusões, ideais primos, ideais radicais, ideais maximais e portanto, temos as seguintes correspondências biunívocas:

$$
\{\text { Conjuntos algébricos contidos em } X\} \underset{I}{\rightleftarrows}\{\text { Ideais radicais de } A(X)\}
$$

$\{$ Conjuntos algébricos irredutíveis contidos em $X\} \underset{V}{\stackrel{I}{\rightleftarrows}}\{$ Ideais primos de $A(X)\}$

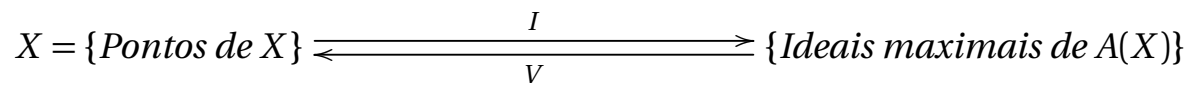

Definição 2.46. A topologia de Zariski é a topologia em $\mathbb{A}_{k}^{n}$ cujos fechados são os conjuntos algébricos afins.

\section{Proposição 2.47.}

1. $\mathbb{A}_{k}^{n}$ com a topologia de Zariski é um espaço topológico Noetheriano, ou seja, se $Y_{1} \supset Y_{2} \supset Y_{3} \supset \cdots e ́$ uma cadeia de fechados, então existe $n$ tal que $Y_{i}=Y_{n}, \forall i \geq n$.

2. Todo conjunto algébrico $X \subset \mathbb{A}_{k}^{n}$ se escreve de maneira única como

$$
X=X_{1} \cup X_{2} \cup \cdots \cup X_{n},
$$


onde cada $X_{i}$ é uma variedade algébrica irredutivel e $X_{i} \not \subset X_{j}, \forall i \neq j$.

Cada $X_{i}$ é chamada de componente irredutivel de X.

Demonstração: Ver o teorema 13 de [Zariski e Samuel (1975)] na página 162.

Observação 2.48. Uma componente irredutível é uma subvariedade irredutível e maximal.

Definição 2.49. Seja X um conjunto algébrico. Para cada cadeia estritamente crescente

$$
X_{0} \subsetneq X_{1} \subsetneq X_{2} \subsetneq \cdots \subsetneq X_{d}
$$

de subconjuntos fechados e irredutíveis de X, o número d é dito comprimento da cadeia e o supremo dos comprimentos dessas cadeias estritamente crescentes de $X$ é chamada de dimensão de $X$ e será denotado por $\operatorname{dim} X$.

Similarmente definiremos a dimensão de um anel.

Definição 2.50. Seja R um anel. Consideremos as cadeias estritamente crescentes de ideais primos de $R$

$$
\boldsymbol{p}_{0} \subsetneq \boldsymbol{p}_{1} \subsetneq \boldsymbol{p}_{2} \subsetneq \cdots \subsetneq \boldsymbol{p}_{r}
$$

A dimensão de Krull de $R$ é o supremo dos comprimentos dessas cadeias e será denotado por $\operatorname{dim}(R)$.

Exemplo 2.51. $\operatorname{dim}\left(k\left[x_{1}, x_{2}, \ldots, x_{n}\right]\right)=n$.

Teorema 2.52. A dimensão de um conjunto algébrico X é igual à dimensão de Krull do anel de coordenadas de $X$, i.e.,

$$
\operatorname{dim} X=\operatorname{dim}(A(X)) .
$$

Definição 2.53. Uma variedade é dita equidimensional se todas suas componentes irredutíveis tem a mesma dimensão.

Exemplo 2.54. Consideremos a variedade algébrica $V(x y, x z) \subset \mathbb{A}_{k}^{3}$. Claramente,

$$
V(x y, x z)=V((x)(y, z))=V(x) \cup V(y, z) .
$$

Agora, observemos que como os ideais $(x) e(y, z)$ são primos, então $V(x)$ e $V(y, z)$ são irredutíveis. Além disso, $\operatorname{dim}(A(V(x)))=\operatorname{dim}(k[y, z])=2 e \operatorname{dim}(A(V(y, z)))=\operatorname{dim}(k[x])=1$.

Geométricamente, notemos que $V(x)$ é o plano y z enquanto $V(y, z)$ é o eixo $x$.

\section{4 Álgebra Homológica}

Nesta seção apresentaremos os conceitos de complexos de módulos, complexos de Koszul e suas homologias, para assim definir interseção completa. Para esta seção fixaremos $R$ um anel associativo.

Definição 2.55. Um complexo $X_{\bullet}$ está dado por sequências $\left(X_{n}\right)_{n \in \mathbb{Z}} e\left(d_{n}^{X}\right)_{n \in \mathbb{Z}}$, em que cada $X_{n}$ é um $R$-módulo e cada $d_{n}^{X}: X_{n} \longrightarrow X_{n-1}$ (diferencial) é um homomorfismo de $R$-módulos, com $d_{n}^{X} d_{n+1}^{X}=0$ para todo $n \in \mathbb{Z}$. Um complexo será representado pela seguinte sequência

$$
X_{\bullet}:=\cdots \longrightarrow X_{n+1} \stackrel{d_{n+1}^{X}}{\longrightarrow} X_{n} \stackrel{d_{n}^{X}}{\longrightarrow} X_{n-1} \longrightarrow \cdots
$$

Definição 2.56. Seja $X$. um complexo. Para cada $n \in \mathbb{Z}$ definimos sua $n$-ésima homologia como

$$
H_{n}\left(X^{\bullet}\right)=\operatorname{ker}\left(d_{n}^{X}\right) / \operatorname{Im}\left(d_{n+1}^{X}\right) .
$$




\subsubsection{Complexos de Koszul}

Comecemos por dar uma ideia básica de complexo de Koszul. Sejam $R$ um anel, $x \in R$ não nulo, $M$ um $R$-módulo e consideremos o homomorfismo de $R$-módulos

$$
\begin{aligned}
x \cdot: M & \longrightarrow M \\
m & \longmapsto x m .
\end{aligned}
$$

Por definição

$$
\operatorname{ker}(x \cdot)=\{m \in M: x m=0\}=: \operatorname{Ann}_{M}(x) .
$$

Além disso,

$$
\operatorname{Im}(x \cdot)=x M e \operatorname{Coker}(x \cdot)=M / x M .
$$

E se considerarmos o complexo

$$
K_{\bullet}(x, M):=\cdots \longrightarrow 0 \longrightarrow \underbrace{M}_{1} \stackrel{x \cdot}{\longrightarrow} \underbrace{M}_{0} \longrightarrow 0 \longrightarrow \cdots
$$

temos que

$$
\begin{gathered}
H_{1}\left(K_{\bullet}(x, M)\right)=\operatorname{ker}(x \cdot)=A n n_{M}(x), \\
H_{0}\left(K_{\bullet}(x, M)\right)=\operatorname{Coker}(x \cdot)=M / x M .
\end{gathered}
$$

Agora introduziremos o conceito de complexo de Koszul, para maiores detalhes ver [Bourbaki (1980)] pag. 147, [Bruns e Herzog (1993)] pag. 39-46 e [Matsumura (1970)] pag. 132- 135.

Sejam $R$ um anel comutativo, $M$ um $R$-módulo e $f: M \longrightarrow R$ uma aplicação $R$-linear. A aplicação

$$
\begin{array}{cl}
M^{n} & \longrightarrow \bigwedge^{n-1} M \\
\left(x_{1}, x_{2}, \ldots, x_{n}\right) & \longmapsto \\
\sum_{i=1}^{n}(-1)^{i+1} f\left(x_{i}\right) x_{1} \wedge x_{2} \wedge \cdots \wedge \widehat{x}_{i} \wedge \cdots \wedge x_{n}
\end{array}
$$

é $n$-linear e alternada, onde $\widehat{x}_{i}$ indica que estamos removendo $x_{i}$ do produto exterior. Pela propriedade universal da $n$-ésima potência exterior (proposição (2.20)) existe um único $d_{n}^{f}: \bigwedge^{n} M \longrightarrow \bigwedge^{n-1} M$ homomorfismo de $R$-módulos, tal que

$$
\begin{array}{ll}
d_{n}^{f}: & \bigwedge^{n} M \\
x_{1} \wedge x_{2} \wedge \ldots \wedge x_{n} & \longrightarrow \bigwedge^{n-1} M \\
\sum_{i=1}^{n}(-1)^{i+1} f\left(x_{i}\right) x_{1} \wedge x_{2} \wedge \cdots \wedge \widehat{x}_{i} \wedge \cdots \wedge x_{n} .
\end{array}
$$

Proposição 2.57. Sejam $R$ umanel comutativo, $M$ um $R$-módulo e $f: M \longrightarrow R$ uma aplicação $R$-linear. A cadeia

$$
K_{\bullet}:=\cdots \stackrel{d_{n+2}^{f}}{\longrightarrow} \bigwedge^{n+1} M \stackrel{d_{n+1}^{f}}{\longrightarrow} \bigwedge^{n} M \stackrel{d_{n}^{f}}{\longrightarrow} \bigwedge^{n-1} M \stackrel{d_{n-1}^{f}}{\longrightarrow} \cdots \stackrel{d_{3}^{f}}{\longrightarrow} \bigwedge^{2} M \stackrel{d_{2}^{f}}{\longrightarrow} M \stackrel{f}{\longrightarrow} R \longrightarrow 0
$$

é um complexo de $R$-módulos.

Demonstração:

$$
\begin{gathered}
f d_{2}^{f}\left(x_{1} \wedge x_{2}\right)=f\left(f\left(x_{1}\right) x_{2}-f\left(x_{2}\right) x_{1}\right)=f\left(x_{1}\right) f\left(x_{2}\right)-f\left(x_{2}\right) f\left(x_{1}\right)=0 \\
d_{n}^{f} d_{n+1}^{f}\left(x_{1} \wedge x_{2} \wedge \cdots \wedge x_{n+1}\right)=d_{n}^{f}\left(\sum_{i=1}^{n+1}(-1)^{i+1} f\left(x_{i}\right) x_{1} \wedge x_{2} \wedge \cdots \wedge \widehat{x}_{i} \wedge \cdots \wedge x_{n+1}\right) \\
=\sum_{i=1}^{n+1}(-1)^{i+1} f\left(x_{i}\right) d_{n}^{f}\left(x_{1} \wedge x_{2} \wedge \cdots \wedge \widehat{x}_{i} \wedge \cdots \wedge x_{n+1}\right) \\
=0
\end{gathered}
$$


Definição 2.58. O complexo acima é chamado de complexo de Koszul de $f$ e denotado por $K_{\bullet}(f)$ (ou simplesmente $K_{.}$) e sua homologia é chamada de homologia de Koszul de $f$ e denotada por $H_{n}(f)$.

Agora se $N$ é um $R$-módulo, então $K_{\bullet}(f, N)$ é o complexo $K_{\bullet}(f) \otimes_{R} N$ chamado de complexo de Koszul de $f$ com coeficientes em $N$. Seus diferenciais são denotados por $d_{n}^{f, N}$, sua homologia por $H_{n}(f, N) e$ será chamada de homologia de Koszul de f com coeficientes em $N$.

Observação 2.59. Seja $M$ um $R$-módulo livre de posto finito e com base $e_{1}, e_{2}, \ldots, e_{n}$. Então, a aplicação $R$-linear $f$ está únicamente determinada pelos valores $x_{i}=f\left(e_{i}\right) \in R$ com $i=1,2, \ldots, n$. Reciprocamente, dada uma sequência $\mathbf{x}=\left\{x_{1}, x_{2}, \ldots, x_{n}\right\}$ em $R$, existe uma aplicação $R$-linear $f$ sobre $M \operatorname{com} f\left(e_{i}\right)=x_{i}$, portanto denotemos

$$
K_{\bullet}(\mathbf{x}):=K_{\bullet}(f) .
$$

Definição 2.60. Para cada sequência $\mathbf{x}=\left\{x_{1}, x_{2}, \ldots, x_{n}\right\}$ num anel comutativo $R$, o complexo $K_{\bullet}(\mathbf{x}) e ́$ chamado de complexo de Koszul de $\mathbf{x}$, sua a homologia de homologia de Koszul de $\mathbf{x}$ e denotada por $H_{n}(\mathbf{x})$.

Agora, se $N$ é um R-módulo, então $K_{\mathbf{\bullet}}(\mathbf{x}, N)$ é o complexo $K_{\mathbf{\bullet}}(\mathbf{x}) \otimes_{R} N$ chamado de complexo de Koszul dex com coeficientes em $N$. Seus diferenciais são denotados por $d_{n}^{\mathbf{x}, N}$, sua homologia por $H_{n}(\mathbf{x}, N)$ e será chamada de homologia de Koszul de x com coeficientes em $N$.

Exemplo 2.61. Se considerarmos $x \neq 0$ um elemento num anel $R$ e a aplicação

$$
\begin{aligned}
f: R & \longrightarrow R \\
r & \longmapsto x r
\end{aligned}
$$

então, para cada $R$-módulo $M$ temos o complexo inicial $K_{\bullet}(x, M):=K_{\bullet}(f, M)$.

Definição 2.62. Sejam $\mathbf{x}=\left\{x_{1}, x_{2}, \ldots, x_{n}\right\}$ uma sequência de elementos num anel $R$ e $M$ um $R$-módulo. Diremos que a sequência é interseção completa para $M$ se

$$
H_{n}(\mathbf{x}, M)=0, \forall n>0 .
$$

Exemplo 2.63. Voltando ao primeiro complexo, temos que a sequência $\mathbf{x}=\{x\}$ é interseção completa sobre o anel $R$ se, e somente se, $x$ não é um divisor de zero sobre $R$.

Com alguns dos resultados da próxima seção, vamos ter uma ideia muito mais clara sobre o que significa que uma sequência seja interseção completa. Para maiores detalhes sobre interseção completa, ver [Bourbaki (1980)] pag. 157-161.

\section{5 Álgebra Comutativa}

Nesta última seção apresentaremos o conceito de sequência regular, algumas propriedades úteis e a relação com equidimensionalidade e interseção completa.

Definição 2.64. Uma sequência $g_{1}, g_{2}, \ldots, g_{t}$ em um anel $R$ é dita regular se a imagem da classe de $g_{i}$ não é divisor de zero e nem invertível em $R /\left(g_{1}, g_{2}, \ldots, g_{i-1}\right)$ para qualquer $i=1,2, \ldots, t$.

Exemplo 2.65. A sequência $x, y(x-1), z(x-1)$ em $k[x, y, z]$ é uma sequência regular, mas a sequência $y(x-1), z(x-1), x$ não é regular em $k[x, y, z]$, pois $\overline{z(x-1)}$ é um divisor de zero em $k[x, y, z] /\langle y(x-1)\rangle$. Para ver isto, basta multiplicar por $\bar{y} \neq 0$ em $k[x, y, z] /\langle y(x-1)\rangle$.

Deste exemplo, podemos concluir que permutações e subsequências de sequências regulares, não necessariamente são regulares.

Proposição 2.66. Sejam $R$ um anel Noetheriano e $g_{1}, g_{2}, \ldots, g_{t}$ uma sequência regular em $R$. Se $R$ é um anel graduado e cada $g_{i}$ é homogêneo de grau positivo, então qualquer permutação de $g_{1}, g_{2}, \ldots, g_{t} e ́$ também regular em $R$. 
Demonstração: Ver teorema 28 em [Matsumura (1970)] página 102 ou [Matsumura (1989)] página 127.

Corolário 2.67. Sejam $R$ um anel Noetheriano e $g_{1}, g_{2}, \ldots, g_{t}$ uma sequência regular em $R$. Se $R$ é um anel graduado e cada $g_{i}$ é homogêneo de grau positivo, então qualquer subsequência de $g_{1}, g_{2}, \ldots, g_{t} e ́$ também regular em $R$.

Demonstração: Segue da proposição (2.66) e da definição de sequência regular.

As seguintes proposições relacionam os conceitos de equidimensionalidade, interseạ̃o completa e sequências regulares.

Proposição 2.68. Seja $g_{1}, g_{2}, \ldots, g_{t}$ uma sequência regular de um anel $R$ e seja $\boldsymbol{e}_{1}, \boldsymbol{e}_{2}, \ldots, \boldsymbol{e}_{t}$ a base canônica do módulo livre $R^{t}$, então a sequência $g_{1}, g_{2}, \ldots, g_{t}$ é interseção completa para $R$, obtendo assim, a sequência exata

$$
\cdots \longrightarrow \bigwedge^{2}\left(R^{t}\right) \stackrel{\partial}{\longrightarrow} R^{t} \stackrel{\eta}{\longrightarrow} R \stackrel{\pi}{\longrightarrow} R /\left(g_{1}, g_{2}, \ldots, g_{t}\right) \longrightarrow 0
$$

chamada de resolução de Koszul, em que $\pi$ é a projeção, $\bigwedge^{i}$ é a i-ésima potência exterior,

$$
\begin{gathered}
\partial\left(\boldsymbol{e}_{i} \wedge \boldsymbol{e}_{j}\right)=g_{i} \boldsymbol{e}_{j}-g_{j} \boldsymbol{e}_{i}, \\
\eta\left(f_{1}, f_{2}, \ldots, f_{t}\right)=\sum_{i=1}^{t} f_{i} g_{i} .
\end{gathered}
$$

Demonstração: Ver proposição 5 de [Bourbaki (1980)] página 157.

Proposição 2.69. Sejam $R$ uma álgebra afim com dimensão de Krull $n$ e $g_{1}, g_{2}, \ldots, g_{t}$ uma sequência em $R \operatorname{com} 0 \leq t \leq n$.

1. Se $R$ é graduada e $g_{1}, g_{2}, \ldots, g_{t}$ homogêneos, então $g_{1}, g_{2}, \ldots, g_{t}$ é uma sequência regular em $R$ se, e somente se, a sequência $g_{1}, g_{2}, \ldots, g_{t}$ é uma interseção completa em $R$.

2. Se $R$ é Cohen-Macaulay ${ }^{1}$, então a sequência $g_{1}, g_{2}, \ldots, g_{t}$ é interseção completa para $R$ se, e somente se, a variedade $V\left(g_{1}, g_{2}, \ldots, g_{t}\right)$ é equidimensional de dimensão $n-t$.

Demonstração: Proposition 2.1 em [Futorny e Ovsienko (2005)].

Proposição 2.70. Sejam $R=k\left[X_{1}, X_{2}, \ldots, X_{n}\right]$ a álgebra de polinômios $e G_{1}, G_{2}, \ldots, G_{t} \in R$. A sequência

$$
X_{1}, X_{2}, \ldots, X_{r}, G_{1}, G_{2}, \ldots, G_{t},
$$

é interseção completa para $R$ se, e somente se, a sequência $g_{1}, g_{2}, \ldots, g_{t}$ é também interseção completa para $k\left[X_{r+1}, X_{r+2}, \ldots, X_{n}\right]$, em que

$$
g_{i}\left(X_{r+1}, X_{r+2}, \ldots, X_{n}\right)=G_{i}\left(0,0, \ldots, 0, X_{r+1}, X_{r+2}, \ldots, X_{n}\right), \forall i=1,2, \ldots, t .
$$

Demonstração: Lema 2.2 de [Futorny e Ovsienko (2005)].

\footnotetext{
${ }^{1}$ Para detalhes sobre anéis Cohen-Macaulay ver [Bruns e Herzog (1993)] página 57 ou em [Matsumura (1989)] página 134. Neste trabalho, só vamos usar o fato que o anel de polinômios é Cohen-Macaulay (teorema 17.7 em [Matsumura (1989)]).
} 


\section{Capítulo 3}

\section{Teorema de Ovsienko e sua versão fraca}

Neste capítulo apresentaremos a subálgebra de Gelfand-Tsetlin $\Gamma$ para $\mathfrak{g l}_{n}$, algumas famílias de geradores de $\Gamma$ e a variedade de Gelfand-Tsetlin como uma variedade algébrica associada a $\Gamma$. Com isto podemos enunciar o Teorema de Ovsienko, o qual fala sobre a equidimensionalidade da variedade de Gelfand-Tsetlin e sua dimensão. Os resultados principais neste capítulo são:

\section{Versão fraca do Teorema de Ovsienko:}

A variedade

$$
V_{n}:=V\left(\sigma_{n 2}, \sigma_{n 3}, \sigma_{n 4}, \ldots, \sigma_{n n}\right) \subset \mathbb{A}_{k}^{\frac{(n+2)(n-1)}{2}},
$$

é equidimensional de dimensão

$$
\operatorname{dim}\left(V_{n}\right)=\frac{(n+2)(n-1)}{2}-(n-1)=\frac{n(n-1)}{2}
$$

em que

$$
\sigma_{n j}=\sum_{n>t_{1}>t_{2}>\cdots>t_{j-1} \geq 1} X_{n t_{1}} X_{t_{1} t_{2}} \cdots X_{t_{j-2} t_{j-1}} X_{t_{j-1} n} ; j=2,3, \ldots, n .
$$

\section{Corolário da versão fraca do Teorema de Ovsienko:}

Todas as componentes regulares da variedade de Gelfand-Tsetlin são isomorfas. Em particular, são isomorfas à componente irredutível

$$
V_{\leq}:=V\left(\left\{X_{i j}: 1 \leq i \leq j \leq n\right\}\right) \subset \mathbb{A}_{k}^{\frac{(n+2)(n-1)}{2}} .
$$

Antes de demonstrar a versão fraca do Teorema de Ovsienko, veremos a prova para os casos particulares $n=2,3,4,5$, com a finalidade de fixar a ideia da demonstração para o caso em que $n$ é arbitrário.

\subsection{Teorema de Ovsienko}

\subsubsection{Subálgebra de Gelfand-Tsetlin para $\mathfrak{g l}_{n}$}

Fixemos $n \in \mathbb{N}$, para cada $i \in\{1,2, \ldots, n\}$ denotemos por $Z_{i}:=Z\left(U\left(\mathfrak{g l}_{i}\right)\right)$ o centro de $U\left(\mathfrak{g l}_{i}\right)$.

Consideremos a álgebra $U\left(\mathfrak{g l}_{n}\right)$ e a base canônica $\left\{E_{i j} \mid 1 \leq i, j \leq n\right\}$ de $\mathfrak{g l}_{n}$. Claramente, para cada $i \in\{1,2, \ldots, n-1\}$ tem-se a identificação de $\mathfrak{g l}_{i}$ como uma subálgebra de $\mathfrak{g l}_{i+1} \mathrm{e} \mathrm{gl}_{n}$, gerada pelo conjunto $\left\{E_{j k} \mid 1 \leq j, k \leq i\right\}$ e portanto, temos a cadeia de subálgebras de Lie de $\mathfrak{g l}_{n}$

$$
\mathfrak{g l}_{1} \subset \mathfrak{g l}_{2} \subset \cdots \subset \mathfrak{g l}_{n-1} \subset \mathfrak{g l}_{n},
$$


a qual induz uma cadeia de subálgebras associativas de $U\left(\mathfrak{g l}_{n}\right)$

$$
U\left(\mathfrak{g l}_{1}\right) \subset U\left(\mathfrak{g l}_{2}\right) \subset \cdots \subset U\left(\mathfrak{g l}_{n-1}\right) \subset U\left(\mathfrak{g l}_{n}\right) .
$$

Definição 3.1. A subálgebra de $U\left(\mathfrak{g l}_{n}\right)$ gerada por $\left\{Z_{1}, Z_{2}, \ldots, Z_{n}\right\}$ é chamada de subálgebra de GelfandTsetlin de $U\left(\mathfrak{g l}_{n}\right)$ e será denotada por $\Gamma$.

Proposição 3.2. Para qualquer $i \in\{1,2, \ldots, n\}$, o centro $Z_{i}$ é uma álgebra de polinômios nas $i$ variáveis $\left\{\gamma_{i j}: j=1,2, \ldots, i\right\}$, com

$$
\gamma_{i j}=\sum_{t_{1}, t_{2}, \ldots, t_{j} \in\{1,2, \ldots, i\}} E_{t_{1} t_{2}} E_{t_{2} t_{3}} \cdots E_{t_{j-1} t_{j}} E_{t_{j} t_{1}} .
$$

Além disso, a subálgebra $\Gamma$ é uma álgebra de polinômios nas $\frac{n(n+1)}{2}$ variáveis $\left\{\gamma_{i j}: 1 \leq j \leq i \leq n\right\}$.

Demonstração: Ver [Želobenko (1973)] página 169.

Observação 3.3. Consideremos a matriz

$$
E:=\left(\begin{array}{cccc}
E_{11} & E_{12} & \cdots & E_{1 n} \\
E_{21} & E_{22} & \cdots & E_{2 n} \\
\vdots & \vdots & \ddots & \vdots \\
E_{n 1} & E_{n 2} & \cdots & E_{n n}
\end{array}\right) \in M_{n}\left(U\left(\mathfrak{g l}_{n}\right)\right)
$$

e para cada $i \in\{1,2, \ldots, n\}$ a $i$-ésima submatriz principal

$$
E_{i}:=\left(\begin{array}{cccc}
E_{11} & E_{12} & \cdots & E_{1 i} \\
E_{21} & E_{22} & \cdots & E_{2 i} \\
\vdots & \vdots & \ddots & \vdots \\
E_{i 1} & E_{i 2} & \cdots & E_{i i}
\end{array}\right) \in M_{i}\left(U\left(\mathfrak{g l}_{i}\right)\right) .
$$

Observemos que para $1 \leq j \leq i \leq n$

$$
\gamma_{i j}=\operatorname{tr}\left(E_{i}^{j}\right)=\sum_{t_{1}=1}^{i} \sum_{t_{2}=1}^{i} \cdots \sum_{t_{j}=1}^{i} E_{t_{1} t_{2}} E_{t_{2} t_{3}} \cdots E_{t_{j-1} t_{j}} E_{t_{j} t_{1}} .
$$

Para visualizar um pouco a dificuldade da combinatória dos geradores, vejamos que os primeiros 9 geradores de $\Gamma$ são:

$$
\begin{aligned}
\gamma_{11}= & E_{11}, \\
\gamma_{21}= & E_{11}+E_{22}, \\
\gamma_{22}= & E_{11}^{2}+E_{12} E_{21}+E_{21} E_{12}+E_{22}^{2}, \\
\gamma_{31}= & E_{11}+E_{22}+E_{33}, \\
\gamma_{32}= & E_{11}^{2}+E_{12} E_{21}+E_{13} E_{31}+E_{21} E_{12}+E_{22}^{2}+E_{23} E_{32}+E_{31} E_{13}+E_{32} E_{23}+E_{33}^{2}, \\
\gamma_{33}= & E_{11}^{3}+E_{11} E_{12} E_{21}+E_{11} E_{13} E_{31}+E_{12} E_{21} E_{11}+E_{12} E_{22} E_{21}+E_{12} E_{23} E_{31}+E_{13} E_{31} E_{11}+ \\
& +E_{13} E_{32} E_{21}+E_{13} E_{33} E_{31}+E_{21} E_{11} E_{12}+E_{21} E_{12} E_{22}+E_{21} E_{13} E_{32}+E_{22} E_{21} E_{12}+E_{22}^{3}+ \\
& +E_{22} E_{23} E_{32}+E_{23} E_{31} E_{12}+E_{23} E_{32} E_{22}+E_{23} E_{33} E_{32}+E_{31} E_{11} E_{13}+E_{31} E_{12} E_{23}+E_{31} E_{13} E_{33}+ \\
& +E_{32} E_{21} E_{13}+E_{32} E_{22} E_{23}+E_{32} E_{23} E_{33}+E_{33} E_{31} E_{13}+E_{33} E_{32} E_{23}+E_{33}^{3}, \\
\gamma_{41}= & E_{11}+E_{22}+E_{33}+E_{44}, \\
\gamma_{42}= & E_{11}^{2}+E_{12} E_{21}+E_{13} E_{31}+E_{14} E_{41}+E_{21} E_{12}+E_{22}^{2}+E_{23} E_{32}+E_{24} E_{42}+E_{31} E_{13}+E_{32} E_{23}+ \\
& +E_{33}^{2}+E_{34} E_{43}+E_{41} E_{14}+E_{42} E_{24}+E_{43} E_{34}+E_{44}^{2},
\end{aligned}
$$




$$
\begin{aligned}
\gamma_{43}= & E_{11}^{3}+E_{11} E_{12} E_{21}+E_{11} E_{13} E_{31}+E_{11} E_{14} E_{41}+E_{12} E_{21} E_{11}+E_{12} E_{22} E_{21}+E_{12} E_{23} E_{31}+E_{12} E_{24} E_{41}+ \\
& +E_{13} E_{31} E_{11}+E_{13} E_{32} E_{21}+E_{13} E_{33} E_{31}+E_{13} E_{34} E_{41}+E_{14} E_{41} E_{11}+E_{14} E_{42} E_{21}+E_{14} E_{43} E_{31}+ \\
& +E_{14} E_{44} E_{41}+E_{21} E_{11} E_{12}+E_{21} E_{12} E_{22}+E_{21} E_{13} E_{32}+E_{21} E_{14} E_{42}+E_{22} E_{21} E_{12}+E_{22}^{3}+E_{22} E_{23} E_{32}+ \\
& +E_{22} E_{24} E_{42}+E_{23} E_{31} E_{12}+E_{23} E_{32} E_{22}+E_{23} E_{33} E_{32}+E_{23} E_{34} E_{42}+E_{24} E_{41} E_{12}+E_{24} E_{42} E_{22}+ \\
& +E_{24} E_{43} E_{32}+E_{24} E_{44} E_{42}+E_{31} E_{11} E_{13}+E_{31} E_{12} E_{23}+E_{31} E_{13} E_{33}+E_{31} E_{14} E_{43}+E_{32} E_{21} E_{13}+ \\
& +E_{32} E_{22} E_{23}+E_{32} E_{23} E_{33}+E_{32} E_{24} E_{43}+E_{33} E_{31} E_{13}+E_{33} E_{32} E_{23}+E_{33}^{3}+E_{33} E_{34} E_{43}+E_{34} E_{41} E_{13}+ \\
& +E_{34} E_{42} E_{23}+E_{34} E_{43} E_{33}+E_{34} E_{44} E_{43}+E_{41} E_{11} E_{14}+E_{41} E_{12} E_{24}+E_{41} E_{13} E_{34}+E_{41} E_{14} E_{44}+ \\
& +E_{42} E_{21} E_{14}+E_{42} E_{22} E_{24}+E_{42} E_{23} E_{34}+E_{42} E_{24} E_{44}+E_{43} E_{31} E_{14}+E_{43} E_{32} E_{24}+E_{43} E_{33} E_{34}+ \\
& +E_{43} E_{34} E_{44}+E_{44} E_{41} E_{14}+E_{44} E_{42} E_{24}+E_{44} E_{43} E_{34}+E_{44}^{3} .
\end{aligned}
$$

\subsubsection{Outros geradores da subálgebra de Gelfand-Tsetlin $\Gamma$}

Como vimos, a combinatória dos geradores é bastante complicada para $n$ suficientemente grande, vejamos outros geradores que vão ser muito úteis. Para mais detalhes ver [Gelfand et al. (1995)] ou [Molev (2007)] páginas 246-250.

Sejam $R$ um anel arbitrário com $1 \in R$ e $A=\left(a_{i j}\right)_{i, j=1}^{n} \in M_{n}(R)$. Denote por $A^{i j}$ a matriz obtida de $A$ tirando a $i$-ésima linha e a $j$-ésima coluna, por $r_{i}^{j}$ a matriz linha $1 \times n-1$ obtida da $i$-ésima linha de $A$ tirando o elemento $a_{i j}$ e por $c_{j}^{i}$ a matriz coluna $n-1 \times 1$ obtida da $j$-ésima coluna de $A$ tirando o elemento $a_{i j}$.

Definição 3.4. Sejam $A=\left(a_{i j}\right)_{i, j=1}^{n} \in M_{n}(R)$ uma matriz $n \times n$ sobre um anel arbitrário $R$ com $1 \in R e$ $i, j \in\{1,2, \ldots, n\}$ tais que, a matriz $A^{i j}$ é invertível. $O$ i $j$-ésimo quasideterminante de $A$ é definido pela fórmula

$$
|A|_{i j}:=a_{i j}-r_{i}^{j}\left(A^{i j}\right)^{-1} c_{j}^{i}
$$

Exemplo 3.5. Considere a matriz

$$
A=\left(\begin{array}{ll}
a_{11} & a_{12} \\
a_{21} & a_{22}
\end{array}\right) .
$$

Então, os 4 quasideterminantes de A são:

$$
\begin{aligned}
& |A|_{11}=a_{11}-r_{1}^{1}\left(A^{11}\right)^{-1} c_{1}^{1}=a_{11}-a_{12} a_{22}^{-1} a_{21}, \\
& |A|_{12}=a_{12}-r_{1}^{2}\left(A^{12}\right)^{-1} c_{2}^{1}=a_{12}-a_{11} a_{21}^{-1} a_{22}, \\
& |A|_{21}=a_{21}-r_{2}^{1}\left(A^{21}\right)^{-1} c_{1}^{2}=a_{21}-a_{22} a_{12}^{-1} a_{11}, \\
& |A|_{22}=a_{22}-r_{2}^{2}\left(A^{22}\right)^{-1} c_{2}^{2}=a_{22}-a_{21} a_{11}^{-1} a_{12} .
\end{aligned}
$$

Para mais detalhes sobre quasideterminante ver [Molev (2007)] páginas 23-27.

Agora, sejam $q$ uma variável formal e $I$ a matriz identidade $n \times n$.

Definição 3.6. Sejam $A=\left(a_{i j}\right)_{i, j=1}^{n} \in M_{n}(R)$ uma matriz $n \times n$ sobre um anel arbitrário $R$ com $1 \in R e$ $i \in\{1,2, \ldots, n\}$ fixo. As funções simétricas elementares não comutativas $\Lambda_{k}^{(i)}$ associadas à matriz A são definidas como os coeficientes da expansão do seguinte quasideterminante

$$
1+\sum_{k \geq 1} \Lambda_{k}^{(i)} q^{k}=|I+q A|_{i i}
$$

Outras famílias de funções simétricas não comutativas de A, chamadas respectivamente completas $S_{k}^{(i)}$, séries de potências de primeiro tipo $\Psi_{k}^{(i)}$ e séries de potências de segundo tipo $\Phi_{k}^{(i)}$ são definidas respectivamente como: 


$$
\begin{gathered}
1+\sum_{k \geq 1} S_{k}^{(i)} q^{k}=|I-q A|_{i i}^{-1}, \\
\sum_{k \geq 1} \Psi_{k}^{(i)} q^{k-1}=|I-q A|_{i i} \frac{d}{d q}|I-q A|_{i i}^{-1}, \\
\sum_{k \geq 1} \Phi_{k}^{(i)} q^{k-1}=-\frac{d}{d q} \log |I-q A|_{i i},
\end{gathered}
$$

em que, a derivada sobre q e o logaritmo são consideradas como operações formais sobre as séries de potências em q com coeficientes no anel R. Em particular, o logaritmo está bem definido sobre as séries quando começam com 1.

Continuando com a matriz $A$, considere o grafo orientado completo $\mathscr{A}$ com $n$ vértices $\{1,2, \ldots, n\}$, e a flecha de $i$ para $j$ será indexada por $a_{i j}$. Cada caminho dirigido no grafo, do vértice $i$ para o vértice $j$ define um monômio da forma

$$
a_{i r_{1}} a_{r_{1} r_{2}} a_{r_{2} r_{3}} \cdots a_{r_{k-2} r_{k-1}} a_{r_{k-1} j}
$$

o qual é obtido considerando o produto de indexações das flechas consecutivas do caminho. O inteiro positivo $k$ é o comprimento do caminho. Um caminho simples, é um caminho tal que $r_{s} \neq i, j$ para todo $s \in\{1,2, \ldots, k-1\}$.

Existe a seguinte descrição das funções simétricas não comutativas da definição (3.6), em termos do grafo $\mathscr{A}$.

\section{Proposição 3.7.}

1. $(-1)^{k-1} \Lambda_{k}^{(i)}$ é a soma de todos os monômios indexando caminhos simples de i para $i$ em $\mathscr{A}$ de comprimento $k$.

2. $S_{k}^{(i)}$ é a soma de todos os monômios indexando caminhos de i para $i$ em $\mathscr{A}$ de comprimento $k$.

3. $\Psi_{k}^{(i)}$ é a soma de todos os monômios indexando caminhos de i para $i$ em $\mathscr{A}$ de comprimento $k$, em que o coeficiente de cada monômio é o comprimento do primeiro retorno ao vértice $i$.

4. $\Phi_{k}^{(i)}$ é a soma de todos os monômios indexando caminhos de i para i em $\mathscr{A}$ de comprimento $k$, em que o coeficiente de cada monômio é o quociente de $k$ pelo número de retornos ao vértice $i$.

Demonstração: Ver [Gelfand et al. (1995)] ou [Molev (2007)] proposição 7.3.1 (página 247).

Agora considere em $\mathfrak{g l}_{n}$, as matrizes $E_{m} \in M_{m}\left(U\left(\mathfrak{g l}_{m}\right)\right)$ para cada $1 \leq m \leq n$ como na observação (3.3) e as funções simétricas não comutativas $\Lambda_{k}^{(m)}, S_{k}^{(m)}, \Psi_{k}^{(m)}$ e $\Phi_{k}^{(m)}$ associadas à matriz $E_{m}+(-m+1) I_{m}$. Pela proposição (3.7), temos expressões explícitas para essas funções. Considere para cada $k \geq 1$

$$
\begin{gathered}
\Lambda_{k}:=\sum_{i_{1}+i_{2}+\cdots+i_{n}=k} \Lambda_{i_{1}}^{(1)} \Lambda_{i_{2}}^{(2)} \cdots \Lambda_{i_{n}}^{(n)}, \\
S_{k}:=\sum_{i_{1}+i_{2}+\cdots+i_{n}=k} S_{i_{1}}^{(1)} S_{i_{2}}^{(2)} \cdots S_{i_{n}}^{(n)}, \\
\Psi_{n k}:=\sum_{i=1}^{n} \Psi_{k}^{(i)}, \\
\Phi_{k}:=\sum_{i=1}^{n} \Phi_{k}^{(i)},
\end{gathered}
$$

em que os índices $i_{j}$ são inteiros não negativos e $\Lambda_{0}^{(j)}=S_{0}^{(j)}=1$. 
Teorema 3.8. Todos os elementos $\Lambda_{k}, S_{k}, \Psi_{k} e \Phi_{k}$ com $k \geq 1$ pertencem ao centro $Z\left(U\left(\mathfrak{g l}_{n}\right)\right)$ da álgebra envolvente universal $U\left(\mathfrak{g l}_{n}\right)$. Além disso, $\Psi_{n k}=\Phi_{k}$ para todo $k$.

Demonstração: Ver [Gelfand et al. (1995)] ou [Molev (2007)] teorema 7.3.2 (página 248) .

Corolário 3.9. Cada família $\left\{\Lambda_{k}\right\}_{k=1}^{n},\left\{S_{k}\right\}_{k=1}^{n} e\left\{\Psi_{n k}\right\}_{k=1}^{n} \operatorname{gera} Z\left(U\left(\mathfrak{g l}_{n}\right)\right)$.

Demonstração: Ver [Gelfand et al. (1995)] ou [Molev (2007)] corolário 7.3.3 (página 250).

Exemplo 3.10.

$$
\begin{gathered}
\Psi_{n 1}=\sum_{i=1}^{n}\left(E_{i i}-i+1\right) \\
\Psi_{n 2}=\sum_{i=1}^{n}\left(E_{i i}-i+1\right)^{2}+2 \sum_{1 \leq t<i \leq n} E_{i t} E_{t i}
\end{gathered}
$$

Corolário 3.11. A subálgebra de Gelfand-Tsetlin $\Gamma$ é gerada por cada família de elementos $\left\{\Lambda_{k}^{(i)}\right\},\left\{S_{k}^{(i)}\right\}$ $e\left\{\Psi_{k}^{(i)}\right\} \operatorname{com} 1 \leq k \leq i \leq n$.

Demonstração: Ver [Gelfand et al. (1995)] ou [Molev (2007)] corolário 7.3.5 (página 250).

\subsubsection{Variedade de Gelfand-Tsetlin para $\mathfrak{g l}_{n}$}

Considere a filtração em $U\left(\mathfrak{g l}_{n}\right)$ vista em (2.2.3). Pelo Teorema de Poincaré-Birkhoff-Witt (2.28)

$$
\operatorname{gr}\left(U\left(\mathfrak{g l}_{n}\right)\right) \cong S\left(\mathfrak{g l}_{n}\right) .
$$

Agora considere gr (Г), a qual pela observação (2.7) é uma subálgebra de $\operatorname{gr}\left(U\left(\mathfrak{g l}_{n}\right)\right)$ e como consequência da proposição (2.18), os geradores $\bar{\gamma}_{i j}$ são polinômios nas variáveis comutativas $\bar{E}_{i j}$

$$
\bar{\gamma}_{i j}=\sum_{t_{1}, t_{2}, \ldots, t_{j} \in\{1,2, \ldots, i\}} \bar{E}_{t_{1} t_{2}} \bar{E}_{t_{2} t_{3}} \cdots \bar{E}_{t_{j-1} t_{j}} \bar{E}_{t_{j} t_{1}} .
$$

Similarmente com os geradores $\bar{\Psi}_{i j}$ do corolário (3.9).

Exemplo 3.12.

$$
\begin{gathered}
\bar{\Psi}_{n 1}=\sum_{i=1}^{n} \bar{E}_{i i} \\
\bar{\Psi}_{n 2}=\sum_{i=1}^{n} \bar{E}_{i i}^{2}+2 \sum_{1 \leq t<i \leq n} \bar{E}_{i t} \bar{E}_{t i}
\end{gathered}
$$

Para facilitar a notação, da proposição (2.18) podemos visualizar $\bar{E}_{i j}$ como variáveis $X_{i j}$. Logo

$$
\bar{\Psi}_{i j}, \bar{\gamma}_{i j} \in k\left[X_{i j}\right]_{i, j=1,2, \ldots, n}
$$

são polinômios nas variáveis comutativas $X_{i j}$.

Definição 3.13. Chamaremos de variedade de Gelfand-Tsetlin para $\mathfrak{g l}_{n}$ a variedade algébrica

$$
V\left(\left\{\bar{\gamma}_{i j}: i=1,2, \ldots, n ; j=1,2, \ldots, i\right\}\right) \subset \mathbb{A}_{k}^{n^{2}}
$$


Da graduação de $U\left(\mathfrak{g l}_{n}\right)$ e corolário (3.9), temos que a variedade de Gelfand-Tsetlin é igual a

$$
V\left(\left\{\bar{\Psi}_{i j}: i=1,2, \ldots, n ; j=1,2, \ldots, i\right\}\right) \subset \mathbb{A}_{k}^{n^{2}},
$$

em que,

$$
\begin{aligned}
& \bar{\Psi}_{i 1}=X_{i i}, \forall i=1,2, \ldots, n, \\
& \bar{\Psi}_{i j}=\sum_{t_{1}, t_{2}, \ldots, t_{j-1} \in\{1,2, \ldots, i-1\}} X_{i t_{1}} X_{t_{1} t_{2}} \cdots X_{t_{j-2} t_{j-1}} X_{t_{j-1}}, \forall i=1,2, \ldots, n ; j=1,2, \ldots, i
\end{aligned}
$$

com $t_{r} \neq t_{s}$ para todo $r \neq s$.

Exemplo 3.14. Alguns desses polinômios $\bar{\Psi}_{i j}$ são:

Ciclos de comprimento 1 :

$$
\bar{\Psi}_{i 1}=X_{i i}, \forall i=1,2, \ldots, n
$$

Ciclos de comprimento 2:

$$
\begin{aligned}
& \bar{\Psi}_{22}=X_{21} X_{12}, \\
& \bar{\Psi}_{32}=X_{31} X_{13}+X_{32} X_{23}, \\
& \bar{\Psi}_{42}=X_{41} X_{14}+X_{42} X_{24}+X_{43} X_{34}, \\
& \quad \vdots \\
& \bar{\Psi}_{n 2}=X_{n 1} X_{1 n}+X_{n 2} X_{2 n}+X_{n 3} X_{3 n}+\cdots+X_{n n-1} X_{n-1 n} .
\end{aligned}
$$

Ciclos de comprimento 3:

$$
\begin{aligned}
\bar{\Psi}_{33} & =X_{31} X_{12} X_{23}+X_{32} X_{21} X_{13} \\
\bar{\Psi}_{43} & =X_{41} X_{12} X_{24}+X_{41} X_{13} X_{34}+X_{42} X_{21} X_{14}+X_{42} X_{23} X_{34}+X_{43} X_{31} X_{14}+X_{43} X_{32} X_{24}, \\
\bar{\Psi}_{53} & =X_{51} X_{12} X_{25}+X_{51} X_{13} X_{35}+X_{51} X_{14} X_{45}+X_{52} X_{21} X_{15}+X_{52} X_{23} X_{35}+X_{52} X_{24} X_{45}+ \\
& +X_{53} X_{31} X_{15}+X_{53} X_{32} X_{25}+X_{53} X_{34} X_{45}+X_{54} X_{41} X_{15}+X_{54} X_{42} X_{25}+X_{54} X_{43} X_{35}
\end{aligned}
$$

Teorema 3.15 (Teorema de Ovsienko).

A variedade de Gelfand-Tsetlin é equidimensional com dimensão

$$
n^{2}-\frac{n(n+1)}{2}=\frac{n(n-1)}{2}
$$

Demonstração: Ver [Ovsienko (2003)].

\subsection{Versão fraca do Teorema de Ovsienko}

Antes de enunciar e provar a versão fraca do Teorema de Ovsienko vamos precisar introduzir algumas notações e do seguinte lema:

Lema 3.16. A variedade de Gelfand-Tsetlin é equidimensional de dimensão

$$
n^{2}-\frac{n(n+1)}{2}=\frac{n(n-1)}{2}
$$

se, e somente se, a variedade

$$
V_{*}:=V\left(\left\{\bar{\Psi}_{i j}: i=2,3, \ldots, n ; j=2,3, \ldots, i\right\}\right) \subset \mathbb{A}_{k}^{n^{2}-n}
$$


é equidimensional de dimensão

$$
\operatorname{dim}\left(V_{*}\right)=n^{2}-n-\left(\frac{n(n+1)}{2}-n\right)=\frac{n(n-1)}{2} .
$$

Demonstração: Isto segue do fato que $\bar{\Psi}_{i 1}=X_{i i}=0, \forall i=1,2, \ldots, n$.

Notação 3.17. Para facilitar a escrita deste capítulo, vamos considerar a seguinte notação:

1. Para um polinômio $P \in k\left[x_{1}, x_{2}, \ldots, x_{n}\right]$ e um conjunto de variáveis $\boldsymbol{X}=\left\{x_{i_{1}}, x_{i_{2}}, \ldots, x_{i_{r}}\right\}$ denotemos

$$
P^{X}:=\left.P\right|_{x_{i_{1}}=x_{i_{2}}=\cdots=x_{i_{r}}=0} .
$$

Ou seja, $P^{X}$ é o polinômio obtido de P fazendo a substituição $x_{i_{1}}=x_{i_{2}}=\cdots=x_{i_{r}}=0$.

2. Para $n \in \mathbb{N}$, definimos o conjunto de variáveis

$$
I_{n}:=\left\{X_{i j}: n \geq i>j \geq 1\right\} \cup\left\{X_{i n}: i=1,2, \ldots, n-1\right\} .
$$

3. Para $t \in \mathbb{N}$ com $t \leq n$, denotemos

$$
I_{n}^{(t)}:=\left\{X_{i j} \in I_{n}: i, j \neq t\right\} .
$$

Agora, podemos apresentar o resultado principal deste capítulo.

Teorema 3.18 (Versão fraca do Teorema de Ovsienko).

A variedade

$$
V_{n}:=V\left(\sigma_{n 2}, \sigma_{n 3}, \sigma_{n 4}, \ldots, \sigma_{n n}\right) \subset \mathbb{A}_{k}^{n^{2}-n-\frac{(n-2)(n-1)}{2}}=\mathbb{A}_{k}^{\frac{(n+2)(n-1)}{2}}
$$

é equidimensional com dimensão

$$
\operatorname{dim}\left(V_{n}\right)=\frac{(n+2)(n-1)}{2}-(n-1)=\frac{n(n-1)}{2},
$$

com

$$
\sigma_{n j}=\sum_{n>t_{1}>t_{2}>\cdots>t_{j-1} \geq 1} X_{n t_{1}} X_{t_{1} t_{2}} \cdots X_{t_{j-2} t_{j-1}} X_{t_{j-1} n} ; j=2,3, \ldots, n .
$$

Observação 3.19. Vale a pena notar que:

1. Se considerarmos a matriz

$$
X=\left(X_{i j}\right)_{i, j=1}^{n},
$$

o conjunto $I_{n}$ está formado pelas entradas da parte triangular estritamente inferior, junto com a última coluna da matriz X e $I_{n}^{(t)}$ está formado pelos elementos de $I_{n}$ tirando a $t$-ésima linha e a $t$-ésima coluna. Intuitivamente, podem ser visualizados como matriz, por exemplo

$$
I_{4}=\left(\begin{array}{llll} 
& & & X_{14} \\
X_{21} & & & X_{24} \\
X_{31} & X_{32} & & X_{34} \\
X_{41} & X_{42} & X_{43} &
\end{array}\right) \quad e \quad I_{5}^{(1)}=\left(\begin{array}{lllll} 
& & & X_{25} \\
X_{32} & & & X_{35} \\
X_{42} & X_{43} & & X_{45} \\
X_{52} & X_{53} & X_{54} &
\end{array}\right) .
$$

2. $\sigma_{n j} \in k\left[I_{n}\right], \quad \forall j=2,3, \ldots, n$. 
A motivação da versão fraca é que acreditamos que ela implica o Teorema de Ovsienko, por exemplo, $V_{2}$ é exatamente a variedade de Gelfand-Tsetlin para $\mathfrak{g l}_{2}$ e a variedade de Gelfand-Tsetlin para $\mathfrak{g l}_{3}$ é igual à união de $V_{3}$ e mais uma variedade isomorfa a $V_{3}$. Mas, o nome de Versão fraca se deve ao fato que $V_{n}$ é uma subvariedade da variedade de Gelfand-Tsetlin para $\mathfrak{g l}_{n}$.

\subsubsection{Casos particulares da Versão fraca do Teorema de Ovsienko}

A prova da versão fraca será por indução sobre $n$. Começaremos provando, os casos particulares $n=2,3,4,5$. O objetivo disso é que $n=2,3$ são casos muitos simples, mas $n=4,5$ são mais interessantes, pois nesses casos se apresenta a técnica que usaremos na demonstração da versão fraca do Teorema de Ovsienko para $n$ arbitrário.

n=2: $V_{2}=V\left(\sigma_{22}\right)=V\left(X_{21} X_{12}\right)=V\left(X_{21}\right) \cup V\left(X_{12}\right) \subset \mathbb{A}_{k}^{2}$ é equidimensional com

$$
\operatorname{dim}\left(V\left(X_{21}\right)\right)=1=\operatorname{dim}\left(V\left(X_{12}\right)\right) .
$$

$\mathbf{n}=3: \quad V_{3}:=V\left(\sigma_{32}, \sigma_{33}\right) \subset \mathbb{A}_{k}^{3^{2}-3-1}=\mathbb{A}_{k}^{5}$

$$
\begin{aligned}
& \sigma_{32}=X_{31} X_{13}+X_{32} X_{23}, \\
& \sigma_{33}=X_{32} X_{21} X_{13} .
\end{aligned}
$$

Vejamos que esta variedade é equidimensional $\operatorname{com} \operatorname{dim}\left(V_{3}\right)=5-2=3$. Como

$$
\sigma_{33}=X_{32} X_{21} X_{13}
$$

então

$$
V_{3}=V\left(\sigma_{32}, X_{32}\right) \cup V\left(\sigma_{32}, X_{21}\right) \cup V\left(\sigma_{32}, X_{13}\right) .
$$

Para $V\left(\sigma_{32}, X_{13}\right) \subset \mathbb{A}_{k}^{5}$, pelas proposições (2.69) e (2.70) basta provar que a variedade

$$
V_{3}^{(1)}:=V\left(\sigma_{32}^{\mathbf{X}}\right) \subset \mathbb{A}_{k}^{4} ; \text { onde } \mathbf{X}=\left\{X_{13}\right\}
$$

é equidimensional com $\operatorname{dim}\left(V_{3}^{(1)}\right)=4-1=3$. O qual é claro, pois

$$
V_{3}^{(1)}=V\left(\sigma_{32}^{\mathbf{x}}\right)=V\left(X_{32} X_{23}\right)=V\left(X_{32}\right) \cup V\left(X_{23}\right) \subset \mathbb{A}_{k}^{4}
$$

é equidimensional com $\operatorname{dim}\left(V\left(X_{32}\right)\right)=3=\operatorname{dim}\left(V\left(X_{23}\right)\right)$.

Para $V\left(\sigma_{32}, X_{21}\right) \subset \mathbb{A}_{k}^{5}$, segue da proposição (2.69) e do corolário (2.67) que basta provar que

$$
V_{3}^{(1)}:=V\left(\sigma_{32}, X_{21}, X_{23}, X_{32}\right) \subset \mathbb{A}_{k}^{5}
$$

é equidimensional com $\operatorname{dim}\left(V_{3}^{(1)}\right)=5-4=1$. Para isto, pelas proposições (2.69) e (2.70) é equivalente provar que a variedade

$$
V_{3}^{(2)}:=V\left(\sigma_{32}^{\mathbf{X}}\right) \subset \mathbb{A}_{k}^{2}, \quad \operatorname{com} \mathbf{X}=\left\{X_{21}, X_{23}, X_{32}\right\}
$$

é equidimensional com $\operatorname{dim}\left(V_{3}^{(2)}\right)=2-1=1$. O qual é claro, pois

$$
V_{3}^{(2)}=V\left(\sigma_{32}^{\mathbf{X}}\right)=V\left(X_{31} X_{13}\right)=V\left(X_{31}\right) \cup V\left(X_{13}\right) \subset \mathbb{A}_{k}^{2}
$$

é equidimensional com $\operatorname{dim}\left(V\left(X_{31}\right)\right)=1=\operatorname{dim}\left(V\left(X_{13}\right)\right)$.

Para $V\left(\sigma_{32}, X_{32}\right) \subset \mathbb{A}_{k}^{5}$, pelas proposições (2.69) e (2.70) basta provar que a variedade

$$
V_{3}^{(1)}:=V\left(\sigma_{32}^{\mathbf{X}}\right) \subset \mathbb{A}_{k}^{4} ; \text { onde } \mathbf{X}=\left\{X_{32}\right\}
$$


é equidimensional com $\operatorname{dim}\left(V_{3}^{(1)}\right)=4-1=3$. O qual segue do fato que

$$
V_{3}^{(1)}=V\left(\sigma_{32}^{\mathbf{X}}\right)=V\left(X_{31} X_{13}\right)=V\left(X_{31}\right) \cup V\left(X_{13}\right) \subset \mathbb{A}_{k}^{4}
$$

é equidimensional com $\operatorname{dim}\left(V\left(X_{31}\right)\right)=3=\operatorname{dim}\left(V\left(X_{13}\right)\right)$.

Finalmente concluimos que a variedade $V_{3}$ é equidimensional com

$$
\operatorname{dim}\left(V_{3}\right)=3 .
$$

$\mathbf{n}=4: V_{4}:=V\left(\sigma_{42}, \sigma_{43}, \sigma_{44}\right) \subset \mathbb{A}_{k}^{4^{2}-4-3}=\mathbb{A}_{k}^{9}$

$$
\begin{aligned}
& \sigma_{42}=X_{41} X_{14}+X_{42} X_{24}+X_{43} X_{34} \\
& \sigma_{43}=X_{42} X_{21} X_{14}+X_{43} X_{31} X_{14}+X_{43} X_{32} X_{24}, \\
& \sigma_{44}=X_{43} X_{32} X_{21} X_{14} .
\end{aligned}
$$

Vejamos que esta variedade é equidimensional $\operatorname{de} \operatorname{dim}\left(V_{4}\right)=9-3=6$. Como

$$
\sigma_{44}=X_{43} X_{32} X_{21} X_{14}
$$

então

$$
V_{4}=V\left(\sigma_{42}, \sigma_{43}, X_{43}\right) \cup \bigcup_{t=2}^{3} V\left(\sigma_{42}, \sigma_{43}, X_{t t-1}\right) \cup V\left(\sigma_{42}, \sigma_{43}, X_{14}\right) .
$$

Para $V\left(\sigma_{42}, \sigma_{43}, X_{14}\right) \subset \mathbb{A}_{k}^{9}$, segue da proposição (2.69) e do corolário (2.67) que basta provar que

$$
V_{4}^{(1)}:=V\left(\sigma_{42}, \sigma_{43}, X_{14}, X_{21}, X_{31}, X_{41}\right) \subset \mathbb{A}_{k}^{9}
$$

é equidimensional com $\operatorname{dim}\left(V_{4}^{(1)}\right)=9-6=3$. Para isso, pelas proposições (2.69) e (2.70) é equivalente provar que ${ }^{1}$

$$
V_{4}^{(2)}:=V\left(\sigma_{42}^{\mathbf{X}}, \sigma_{43}^{\mathbf{X}}\right) \subset \mathbb{A}_{k}^{5}, \quad \mathbf{X}=\left\{X_{14}, X_{21}, X_{31}, X_{41}\right\}
$$

é equidimensional com $\operatorname{dim}\left(V_{4}^{(2)}\right)=3$. Mas,

$$
\begin{aligned}
& \sigma_{42}^{\mathbf{X}}=X_{42} X_{24}+X_{43} X_{34}, \\
& \sigma_{43}^{\mathbf{X}}=X_{43} X_{32} X_{24} .
\end{aligned}
$$

Agora, tomemos o isomorfismo de $k$-álgebras

$$
\begin{aligned}
\varphi: k\left[I_{3}\right] & \longrightarrow k\left[I_{4}^{(1)}\right] \\
X_{i j} & \longmapsto \varphi\left(X_{i j}\right):=X_{i+1, j+1},
\end{aligned}
$$

isto é,

$$
\left(\begin{array}{lll} 
& & X_{13} \\
X_{21} & & X_{23} \\
X_{31} & X_{32} &
\end{array}\right) \longmapsto\left(\begin{array}{lll} 
& & X_{24} \\
X_{32} & & X_{34} \\
X_{42} & X_{43} &
\end{array}\right)
$$

temos que

$$
\begin{aligned}
& \varphi\left(\sigma_{32}\right)=\varphi\left(X_{31} X_{13}+X_{32} X_{23}\right)=X_{42} X_{24}+X_{43} X_{34} \\
& \varphi\left(\sigma_{33}\right)=\varphi\left(X_{32} X_{21} X_{13}\right)=X_{43} X_{32} X_{24} .
\end{aligned}
$$

${ }^{1}$ Observemos que se visualizarmos $I_{4}$ como matriz, então $\mathbf{X}$ está formado pela primeira linha e a primeira coluna de $I_{4}$. 
Portanto $V_{4}^{(2)} \cong V_{3}$, que é equidimensional com dimensão

$$
\operatorname{dim}\left(V_{4}^{(2)}\right)=\operatorname{dim}\left(V_{3}\right)=3 .
$$

Para $V\left(\sigma_{42}, \sigma_{43}, X_{t t-1}\right) \subset \mathbb{A}_{k}^{9} \operatorname{com} t \in\{2,3\}$, segue da proposição (2.69) e do corolário (2.67) que é suficiente provar que

$$
\begin{aligned}
& V_{4}^{(1)}:=V\left(\sigma_{42}, \sigma_{43}, X_{21}, X_{24}, X_{32}, X_{42}\right) \subset \mathbb{A}_{k}^{9} \quad(\text { se } t=2), \\
& V_{4}^{(1)}:=V\left(\sigma_{42}, \sigma_{43}, X_{32}, X_{31}, X_{34}, X_{43}\right) \subset \mathbb{A}_{k}^{9} \quad(\text { se } t=3)
\end{aligned}
$$

é equidimensional com $\operatorname{dim}\left(V_{4}^{(1)}\right)=9-6=3$. Para isso, pelas proposições (2.69) e (2.70) é equivalente provar que

$$
V_{4}^{(2)}:=V\left(\sigma_{42}^{\mathbf{X}}, \sigma_{43}^{\mathbf{X}}\right) \subset \mathbb{A}_{k}^{5}
$$

é equidimensional com $\operatorname{dim}\left(V_{4}^{(2)}\right)=5-2=3$, em que ${ }^{2}$

$$
\begin{aligned}
& \mathbf{X}=\left\{X_{21}, X_{24}, X_{32}, X_{42}\right\} \quad(\text { se } t=2), \\
& \mathbf{X}=\left\{X_{32}, X_{31}, X_{34}, X_{43}\right\} \quad(\text { se } t=3) .
\end{aligned}
$$

Observemos que

\begin{tabular}{l||l|l|} 
& $\mathrm{t}=2$ & $\mathrm{t}=3$ \\
\hline \hline$\sigma_{42}^{\mathbf{X}}$ & $X_{41} X_{14}+X_{43} X_{34}$ & $X_{41} X_{14}+X_{42} X_{24}$ \\
$\sigma_{43}^{\mathbf{X}}$ & $X_{43} X_{31} X_{14}$ & $X_{42} X_{21} X_{14}$ \\
\hline
\end{tabular}

e se considerarmos o isomorfismo de $k$-álgebras

$$
\begin{aligned}
\varphi: k\left[I_{3}\right] & \longrightarrow k\left[I_{4}^{(t)}\right] \\
X_{i j} & \longrightarrow \varphi\left(X_{i j}\right):=\left\{\begin{array}{cc}
X_{i j} ; & \text { se } i, j<t \\
X_{i+1, j} ; & \text { se } j<t \leq i \\
X_{i, j+1} ; & \text { se } i<t \leq j \\
X_{i+1, j+1} ; & \text { se } t \leq i, j
\end{array},\right.
\end{aligned}
$$

isto é,

$$
\begin{aligned}
&\left(\begin{array}{lll} 
& & X_{13} \\
X_{21} & & X_{23} \\
X_{31} & X_{32} &
\end{array}\right)\left.\longmapsto\left(\begin{array}{lll} 
& & X_{14} \\
X_{31} & & X_{34} \\
X_{41} & X_{43} &
\end{array}\right) \quad \text { (se } t=2\right), \\
&\left(\begin{array}{lll}
X_{21} & & X_{23} \\
X_{31} & X_{32} &
\end{array}\right) \longmapsto\left(\begin{array}{lll} 
& & X_{14} \\
X_{21} & & X_{24} \\
X_{41} & X_{42} &
\end{array}\right) \quad \text { (se } t=3 \text { ), }
\end{aligned}
$$

temos que

\begin{tabular}{l||l|l|} 
& $\mathrm{t}=2$ & $\mathrm{t}=3$ \\
\hline \hline$\varphi\left(\sigma_{32}\right)$ & $\varphi\left(X_{31} X_{13}+X_{32} X_{23}\right)=X_{41} X_{14}+X_{43} X_{34}$ & $\varphi\left(X_{31} X_{13}+X_{32} X_{23}\right)=X_{41} X_{14}+X_{42} X_{24}$ \\
$\varphi\left(\sigma_{33}\right)$ & $\varphi\left(X_{32} X_{21} X_{13}\right)=X_{43} X_{31} X_{14}$ & $\varphi\left(X_{32} X_{21} X_{13}\right)=X_{42} X_{21} X_{14}$ \\
\hline
\end{tabular}

Assim,

$$
\begin{aligned}
& \varphi\left(\sigma_{32}\right)=\sigma_{42}^{\mathbf{X}}, \\
& \varphi\left(\sigma_{33}\right)=\sigma_{43}^{\mathbf{X}} .
\end{aligned}
$$

${ }^{2}$ Observemos que se visualizarmos $I_{4}$ como matriz, então $\mathbf{X}$ está formado pela $t$-ésima linha e a $t$-ésima coluna de $I_{4}$. 
Portanto $V_{4}^{(2)} \cong V_{3}$, a qual é equidimensional com dimensão $\operatorname{dim}\left(V_{4}^{(2)}\right)=\operatorname{dim}\left(V_{3}\right)=3$.

Para $V\left(\sigma_{42}, \sigma_{43}, X_{43}\right) \subset \mathbb{A}_{k}^{9}$, segue da proposição (2.69) e do corolário (2.67) que é suficiente provar que ${ }^{3}$

$$
V_{4}^{(1)}:=V\left(\sigma_{42}, \sigma_{43}, X_{43}, X_{31}, X_{32}, X_{34}\right) \subset \mathbb{A}_{k}^{9}
$$

é equidimensional com $\operatorname{dim}\left(V_{4}^{(1)}\right)=9-6=3$. Para isso, pelas proposições (2.69) e (2.70) é equivalente provar que ${ }^{4}$

$$
V_{4}^{(2)}:=V\left(\sigma_{42}^{\mathbf{X}}, \sigma_{43}^{\mathbf{X}}\right) \subset \mathbb{A}_{k}^{9-4}=\mathbb{A}_{k}^{5} \operatorname{com} \mathbf{X}=\left\{X_{43}, X_{31}, X_{32}, X_{34}\right\}
$$

é equidimensional com $\operatorname{dim}\left(V_{4}^{(2)}\right)=5-2=3$. Observemos que

$$
\begin{aligned}
& \sigma_{42}^{\mathbf{X}}=X_{41} X_{14}+X_{42} X_{24}, \\
& \sigma_{43}^{\mathbf{X}}=X_{42} X_{21} X_{14} .
\end{aligned}
$$

Agora, consideremos o isomomorfismo de $k$-álgebras

$$
\begin{aligned}
\varphi: k\left[I_{3}\right] & \longrightarrow k\left[I_{4}^{(3)}\right] \\
X_{i j} & \longrightarrow \varphi\left(X_{i j}\right):=\left\{\begin{array}{cc}
X_{i j} ; & \text { se } i, j \neq 3 \\
X_{4 j} ; & \text { se } i=3 \\
X_{i 4} ; & \text { se } j=3
\end{array},\right.
\end{aligned}
$$

isto é,

$$
\left(\begin{array}{lll} 
& & X_{13} \\
X_{21} & & X_{23} \\
X_{31} & X_{32} &
\end{array}\right) \longmapsto\left(\begin{array}{lll} 
& & X_{14} \\
X_{21} & & X_{24} \\
X_{41} & X_{42} &
\end{array}\right) .
$$

Logo

$$
\begin{aligned}
& \varphi\left(\sigma_{32}\right)=\varphi\left(X_{31} X_{13}+X_{32} X_{23}\right)=X_{41} X_{14}+X_{42} X_{24}=\sigma_{42}^{\mathbf{X}}, \\
& \varphi\left(\sigma_{33}\right)=\varphi\left(X_{32} X_{21} X_{13}\right)=X_{42} X_{21} X_{14}=\sigma_{42}^{\mathbf{X}} .
\end{aligned}
$$

Portanto $V_{4}^{(2)} \cong V_{3}$, a qual é equidimensional $\operatorname{com} \operatorname{dim}\left(V_{4}^{(2)}\right)=\operatorname{dim}\left(V_{3}\right)=3$.

Finalmente concluimos que a variedade $V_{4}$ é equidimensional com

$$
\operatorname{dim}\left(V_{4}\right)=6 .
$$

$\mathbf{n}=\mathbf{5 :} \quad V_{5}:=V\left(\sigma_{52}, \sigma_{53}, \sigma_{54}, \sigma_{55}\right) \subset \mathbb{A}_{k}^{5^{2}-5-6}=\mathbb{A}_{k}^{14}$

$$
\begin{aligned}
& \sigma_{52}=X_{51} X_{15}+X_{52} X_{25}+X_{53} X_{35}+X_{54} X_{45}, \\
& \sigma_{53}=X_{52} X_{21} X_{15}+X_{53} X_{31} X_{15}+X_{53} X_{32} X_{25}+X_{54} X_{41} X_{15}+X_{54} X_{42} X_{25}+X_{54} X_{43} X_{35}, \\
& \sigma_{54}=X_{53} X_{32} X_{21} X_{15}+X_{54} X_{42} X_{21} X_{15}+X_{54} X_{43} X_{31} X_{15}+X_{54} X_{43} X_{32} X_{25}, \\
& \sigma_{55}=X_{54} X_{43} X_{32} X_{21} X_{15} .
\end{aligned}
$$

Provemos que esta variedade é equidimensional $\operatorname{de} \operatorname{dim}\left(V_{5}\right)=14-4=10$. Como

$$
\sigma_{55}=X_{54} X_{43} X_{32} X_{21} X_{15}
$$

\footnotetext{
${ }^{3}$ Observemos que aqui não podemos usar a mesma técnica dos dois casos anteriores, porque se acrescentarmos a quarta coluna ou a quarta linha estaríamos eliminando todas as equações, i.e., $\sigma_{42}^{\mathbf{X}}=\sigma_{43}^{\mathbf{X}}=0$ e portanto a sequência $\sigma_{42}^{\mathbf{X}}, \sigma_{43}^{\mathbf{X}}$ não seria uma sequência regular. Neste caso, só acrescentaremos a terceira linha de $I_{4}$.

${ }^{4}$ Observemos que se visualizarmos $I_{4}$ como matriz, então X está formado pela terceira linha e a terceira coluna de $I_{4}$.
} 
então,

$$
V_{5}=V\left(\sigma_{52}, \sigma_{53}, \sigma_{54}, X_{54}\right) \cup \bigcup_{t=2}^{4} V\left(\sigma_{52}, \sigma_{53}, \sigma_{54}, X_{t t-1}\right) \cup V\left(\sigma_{52}, \sigma_{53}, \sigma_{54}, X_{15}\right)
$$

Para $V\left(\sigma_{52}, \sigma_{53}, \sigma_{54}, X_{15}\right) \subset \mathbb{A}_{k}^{14}$, segue da proposição (2.69) e do corolário (2.67) que é suficiente provar que a variedade

$$
V_{5}^{(1)}:=V\left(\sigma_{52}, \sigma_{53}, \sigma_{54}, X_{15}, X_{21}, X_{31}, X_{41}, X_{51}\right) \subset \mathbb{A}_{k}^{14}
$$

é equidimensional com $\operatorname{dim}\left(V_{5}^{(1)}\right)=14-8=6$. Para isso, pelas proposições (2.69) e (2.70) é equivalente provar que

$$
V_{5}^{(2)}:=V\left(\sigma_{52}^{\mathbf{X}}, \sigma_{53}^{\mathbf{X}}, \sigma_{54}^{\mathbf{X}}\right) \subset \mathbb{A}_{k}^{9}, \quad \mathbf{X}=\left\{X_{15}, X_{21}, X_{31}, X_{41}, X_{51}\right\}
$$

é equidimensional com $\operatorname{dim}\left(V_{5}^{(2)}\right)=9-3=6$. Observemos que

$$
\begin{aligned}
& \sigma_{52}^{\mathbf{X}}=X_{52} X_{25}+X_{53} X_{35}+X_{54} X_{45}, \\
& \sigma_{53}^{\mathbf{X}}=X_{53} X_{32} X_{25}+X_{54} X_{42} X_{25}+X_{54} X_{43} X_{35}, \\
& \sigma_{54}^{\mathbf{X}}=X_{54} X_{43} X_{32} X_{25} .
\end{aligned}
$$

Agora, considerando o isomorfismo de $k$-álgebras

$$
\begin{aligned}
\varphi: k\left[I_{4}\right] & \longrightarrow k\left[I_{5}^{(1)}\right] \\
X_{i j} & \longrightarrow \varphi\left(X_{i j}\right):=X_{i+1, j+1}
\end{aligned},
$$

isto é,

$$
\left(\begin{array}{lllll} 
& & & X_{14} \\
X_{21} & & & X_{24} \\
X_{31} & X_{32} & & X_{34} \\
X_{41} & X_{42} & X_{43} &
\end{array}\right) \longmapsto\left(\begin{array}{lllll} 
& & & X_{25} \\
X_{32} & & & X_{35} \\
X_{42} & X_{43} & & X_{45} \\
X_{52} & X_{53} & X_{54} &
\end{array}\right),
$$

temos que

$$
\begin{aligned}
& \varphi\left(\sigma_{42}\right)=\varphi\left(X_{41} X_{14}+X_{42} X_{24}+X_{43} X_{34}\right)=X_{52} X_{25}+X_{53} X_{35}+X_{54} X_{45}=\sigma_{52}^{\mathbf{X}}, \\
& \varphi\left(\sigma_{43}\right)=\varphi\left(X_{42} X_{21} X_{14}+X_{43} X_{31} X_{14}+X_{43} X_{32} X_{24}\right)=X_{53} X_{32} X_{25}+X_{54} X_{42} X_{25}+X_{54} X_{43} X_{35}=\sigma_{53} \mathbf{X} \\
& \varphi\left(\sigma_{44}\right)=\varphi\left(X_{43} X_{32} X_{21} X_{14}\right)=X_{54} X_{43} X_{32} X_{25}=\sigma_{54}^{\mathbf{X}} .
\end{aligned}
$$

Portanto $V_{5}^{(2)} \cong V_{4}$, a qual é equidimensional com dimensão $\operatorname{dim}\left(V_{5}^{(2)}\right)=\operatorname{dim}\left(V_{4}\right)=6$.

Para $V\left(\sigma_{52}, \sigma_{53}, \sigma_{54}, X_{t t-1}\right) \subset \mathbb{A}_{k}^{14} \operatorname{com} t \in\{2,3,4\}$, segue da proposição (2.69) e do corolário (2.67) que é suficiente provar que ${ }^{5}$

$$
\begin{aligned}
& V_{5}^{(1)}:=V\left(\sigma_{52}, \sigma_{53}, \sigma_{54}, X_{21}, X_{25}, X_{32}, X_{42}, X_{52}\right) \subset \mathbb{A}_{k}^{14}, \quad \text { se } t=2 \\
& V_{5}^{(1)}:=V\left(\sigma_{52}, \sigma_{53}, \sigma_{54}, X_{32}, X_{31}, X_{35}, X_{43}, X_{53}\right) \subset \mathbb{A}_{k}^{14}, \quad \text { se } t=3 \\
& V_{5}^{(1)}:=V\left(\sigma_{52}, \sigma_{53}, \sigma_{54}, X_{43}, X_{41}, X_{42}, X_{45}, X_{54}\right) \subset \mathbb{A}_{k}^{14}, \quad \text { se } t=4
\end{aligned}
$$

é equidimensional com $\operatorname{dim}\left(V_{5}^{(1)}\right)=14-8=6$. Para isso, pelas proposições (2.69) e (2.70) é equivalente mostrar que

$$
V_{5}^{(2)}:=V\left(\sigma_{52}^{\mathbf{X}}, \sigma_{53}^{\mathbf{X}}, \sigma_{54}^{\mathbf{X}}\right) \subset \mathbb{A}_{k}^{9}
$$

\footnotetext{
${ }^{5}$ Aqui estamos acrescentando a $t$-ésima linha e a $t$-ésima coluna da matriz $I_{5}$.
} 
é equidimensional com $\operatorname{dim}\left(V_{5}^{(2)}\right)=9-3=6$, em que

$$
\begin{array}{ll}
\mathbf{X}=\left(X_{21}, X_{25}, X_{32}, X_{42}, X_{52}\right), & \text { se } t=2 \\
\mathbf{X}=\left(X_{32}, X_{31}, X_{35}, X_{43}, X_{53}\right), & \text { se } t=3 \\
\mathbf{X}=\left(X_{43}, X_{41}, X_{42}, X_{45}, X_{54}\right), & \text { se } t=4 .
\end{array}
$$

Agora, observemos que

\begin{tabular}{c||l|l|} 
& \multicolumn{1}{|c|}{$t=2$} & \multicolumn{1}{|c|}{$t=3$} \\
\hline \hline$\sigma_{52}^{\mathbf{X}}$ & $X_{51} X_{15}+X_{53} X_{35}+X_{54} X_{45}$ & $X_{51} X_{15}+X_{52} X_{25}+X_{54} X_{45}$ \\
$\sigma_{53}^{\mathbf{X}}$ & $X_{53} X_{31} X_{15}+X_{54} X_{41} X_{15}+X_{54} X_{43} X_{35}$ & $X_{52} X_{21} X_{15}+X_{54} X_{41} X_{15}+X_{54} X_{42} X_{25}$ \\
$\sigma_{54}^{\mathbf{X}}$ & $X_{54} X_{43} X_{31} X_{15}$ & $X_{54} X_{42} X_{21} X_{15}$ \\
\hline
\end{tabular}

\begin{tabular}{l||l|} 
& \multicolumn{1}{||}{$t=4$} \\
\hline \hline$\sigma_{52}^{\mathbf{X}}$ & $X_{51} X_{15}+X_{52} X_{25}+X_{53} X_{35}$ \\
$\sigma_{53}^{\mathbf{X}}$ & $X_{52} X_{21} X_{15}+X_{53} X_{31} X_{15}+X_{53} X_{32} X_{25}$ \\
$\sigma_{54}^{\mathbf{X}}$ & $X_{53} X_{32} X_{21} X_{15}$ \\
\hline
\end{tabular}

Agora, consideremos o isomorfismo de $k$-álgebras ${ }^{6}$

$$
\begin{array}{r}
\varphi: k\left[I_{4}\right] \quad \longrightarrow \quad k\left[I_{5}^{(t)}\right] \\
X_{i j} \quad \longrightarrow \varphi\left(X_{i j}\right):=\left\{\begin{array}{cc}
X_{i j} ; & \text { se } i, j<t \\
X_{i+1, j} ; & \text { se } j<t \leq i \\
X_{i, j+1} ; & \text { se } i<t \leq j \\
X_{i+1, j+1} ; & \text { se } t \leq i, j
\end{array},\right.
\end{array}
$$

isto é,

$$
\begin{aligned}
& \left(\begin{array}{llll} 
& & & X_{14} \\
X_{21} & & & X_{24} \\
X_{31} & X_{32} & & X_{34} \\
X_{41} & X_{42} & X_{43} &
\end{array}\right) \longmapsto\left(\begin{array}{llll} 
& & & X_{15} \\
X_{31} & & & X_{35} \\
X_{41} & X_{43} & & X_{45} \\
X_{51} & X_{53} & X_{54} &
\end{array}\right) \text {, se } t=2 \\
& \left(\begin{array}{lllll} 
& & & X_{14} \\
X_{21} & & & X_{24} \\
X_{31} & X_{32} & & X_{34} \\
X_{41} & X_{42} & X_{43} &
\end{array}\right) \longmapsto\left(\begin{array}{llll} 
& \multicolumn{3}{c}{X_{15}} \\
X_{21} & & & X_{25} \\
X_{41} & X_{42} & & X_{45} \\
X_{51} & X_{52} & X_{54} &
\end{array}\right) \text {, se } t=3 \\
& \left(\begin{array}{llll} 
& & & X_{14} \\
X_{21} & & & X_{24} \\
X_{31} & X_{32} & & X_{34} \\
X_{41} & X_{42} & X_{43} &
\end{array}\right) \longmapsto\left(\begin{array}{llll} 
& & & X_{15} \\
X_{21} & & & X_{25} \\
X_{31} & X_{32} & & X_{35} \\
X_{51} & X_{52} & X_{53} &
\end{array}\right) \text {, se } t=4
\end{aligned}
$$

segue que

\begin{tabular}{c||l|l|} 
& \multicolumn{1}{|c|}{$t=2$} & \multicolumn{1}{|c|}{$t=3$} \\
\hline \hline$\varphi\left(\sigma_{42}\right)$ & $X_{51} X_{15}+X_{53} X_{35}+X_{54} X_{45}$ & $X_{51} X_{15}+X_{52} X_{25}+X_{54} X_{45}$ \\
$\varphi\left(\sigma_{43}\right)$ & $X_{53} X_{31} X_{15}+X_{54} X_{41} X_{15}+X_{54} X_{43} X_{35}$ & $X_{52} X_{21} X_{15}+X_{54} X_{41} X_{15}+X_{54} X_{42} X_{25}$ \\
$\varphi\left(\sigma_{44}\right)$ & $X_{54} X_{43} X_{31} X_{15}$ & $X_{54} X_{42} X_{21} X_{15}$ \\
\hline
\end{tabular}

\footnotetext{
${ }^{6}$ Aqui estamos associando a matriz $I_{4}$ com a matriz $I_{5}^{(t)}$, i.e., com a matriz $I_{5}$ tirando a $t$-ésima linha e a $t$-ésima coluna.
} 


\begin{tabular}{c||c|} 
& \multicolumn{1}{||}{$t=4$} \\
\hline \hline$\varphi\left(\sigma_{42}\right)$ & $X_{51} X_{15}+X_{52} X_{25}+X_{53} X_{35}$ \\
$\varphi\left(\sigma_{43}\right)$ & $X_{52} X_{21} X_{15}+X_{53} X_{31} X_{15}+X_{53} X_{32} X_{25}$ \\
$\varphi\left(\sigma_{44}\right)$ & $X_{53} X_{32} X_{21} X_{15}$ \\
\hline
\end{tabular}

Assim

$$
\begin{aligned}
& \varphi\left(\sigma_{42}\right)=\sigma_{52}^{\mathbf{X}}, \\
& \varphi\left(\sigma_{43}\right)=\sigma_{53}^{\mathbf{X}}, \\
& \varphi\left(\sigma_{44}\right)=\sigma_{54}^{\mathbf{X}} .
\end{aligned}
$$

Portanto $V_{5}^{(2)} \cong V_{4}$, a qual é equidimensional com dimensão $\operatorname{dim}\left(V_{5}^{(2)}\right)=\operatorname{dim}\left(V_{4}\right)=6$.

Para $V\left(\sigma_{52}, \sigma_{53}, \sigma_{54}, X_{54}\right) \subset \mathbb{A}_{k}^{14}$, segue da proposição (2.69) e do corolário (2.67) que basta provar que $^{7}$

$$
V_{5}^{(1)}:=V\left(\sigma_{52}, \sigma_{53}, \sigma_{54}, X_{54}, X_{41}, X_{42}, X_{43}, X_{45}\right) \subset \mathbb{A}_{k}^{14}
$$

é equidimensional com $\operatorname{dim}\left(V_{5}^{(1)}\right)=14-8=6$. Para isso, pelas proposições (2.69) e (2.70) é equivalente mostrar que

$$
V_{5}^{(2)}:=V\left(\sigma_{52}^{\mathbf{X}}, \sigma_{53}^{\mathbf{X}}, \sigma_{54}^{\mathbf{X}}\right) \subset \mathbb{A}_{k}^{14-5}=\mathbb{A}_{k}^{9} \quad \text { com } \quad \mathbf{X}=\left\{X_{54}, X_{41}, X_{42}, X_{43}, X_{45}\right\}
$$

é equidimensional com $\operatorname{dim}\left(V_{5}^{(2)}\right)=9-3=6$. Observemos que

$$
\begin{aligned}
& \sigma_{52}^{\mathbf{x}}=X_{51} X_{15}+X_{52} X_{25}+X_{53} X_{35}, \\
& \sigma_{53}^{\mathbf{x}}=X_{52} X_{21} X_{15}+X_{53} X_{31} X_{15}+X_{53} X_{32} X_{25}, \\
& \sigma_{54}^{\mathbf{X}}=X_{53} X_{32} X_{21} X_{15} .
\end{aligned}
$$

Agora, consideremos o isomomorfismo de $k$-álgebras ${ }^{8}$

$$
\begin{aligned}
\varphi: k\left[I_{4}\right] & \longrightarrow k\left[I_{5}^{(4)}\right] \\
X_{i j} & \longrightarrow \varphi\left(X_{i j}\right):=\left\{\begin{array}{cc}
X_{i j} ; & \text { se } i, j \neq 4 \\
X_{5 j} ; & \text { se } i=4 \\
X_{i 5} ; & \text { se } j=4
\end{array},\right.
\end{aligned}
$$

isto é,

$$
\left(\begin{array}{lllll} 
& & & X_{14} \\
X_{21} & & & X_{24} \\
X_{31} & X_{32} & & X_{34} \\
X_{41} & X_{42} & X_{43} &
\end{array}\right) \longmapsto\left(\begin{array}{lllll} 
& & & X_{15} \\
X_{21} & & & X_{25} \\
X_{31} & X_{32} & & X_{35} \\
X_{51} & X_{52} & X_{53} &
\end{array}\right) .
$$

Assim

$$
\begin{aligned}
& \varphi\left(\sigma_{42}\right)=\varphi\left(X_{41} X_{14}+X_{42} X_{24}+X_{43} X_{34}\right)=X_{51} X_{15}+X_{52} X_{25}+X_{53} X_{35}=\sigma_{52}^{\mathbf{x}}, \\
& \varphi\left(\sigma_{43}\right)=\varphi\left(X_{42} X_{21} X_{14}+X_{43} X_{31} X_{14}+X_{43} X_{32} X_{24}\right)=X_{52} X_{21} X_{15}+X_{53} X_{31} X_{15}+X_{53} X_{32} X_{25}=\sigma_{53}^{\mathbf{x}}, \\
& \varphi\left(\sigma_{44}\right)=\varphi\left(X_{43} X_{32} X_{21} X_{14}\right)=X_{53} X_{32} X_{21} X_{15}=\sigma_{54}^{\mathbf{X}} .
\end{aligned}
$$

Portanto $V_{5}^{(2)} \cong V_{4}$, a qual é equidimensional $\operatorname{com} \operatorname{dim}\left(V_{5}^{(2)}\right)=\operatorname{dim}\left(V_{4}\right)=6$.

\footnotetext{
${ }^{7}$ Observemos que aqui não podemos usar a mesma técnica dos dois casos anteriores, porque se acrescentarmos a quinta coluna ou a quinta linha estaríamos eliminando todas as equações, i.e., $\sigma_{52}^{\mathbf{X}}=\sigma_{53}^{\mathbf{X}}=\sigma_{54}^{\mathbf{X}}=0$ e portanto a sequência $\sigma_{52}^{\mathbf{X}}, \sigma_{53}^{\mathbf{X}}, \sigma_{54}^{\mathbf{X}}$ não seria uma sequência regular. Neste caso, só acrescentamos a quarta linha de $I_{5}$.

${ }^{8}$ Aqui estamos só associando $I_{4} \operatorname{com} I_{5}^{(4)}$, i.e., tirando a quarta coluna e a quarta linha da matriz $I_{5}$.
} 
Finalmente, dos casos anteriores concluimos que a variedade $V_{5}$ é equidimensional com

$$
\operatorname{dim}\left(V_{5}\right)=10
$$

\subsubsection{Demonstração da versão fraca do Teorema de Ovsienko}

Demonstração: Provemos por indução sobre $n$. Claramente, como visto anteriormente

$$
V_{2}=V\left(\sigma_{22}\right)=V\left(X_{21} X_{12}\right)=V\left(X_{21}\right) \cup V\left(X_{12}\right) \subset \mathbb{A}_{k}^{2}
$$

é equidimensional com

$$
\operatorname{dim}\left(V\left(X_{21}\right)\right)=1=\operatorname{dim}\left(V\left(X_{12}\right)\right) .
$$

Suponhamos que

$$
V_{n-1}=V\left(\sigma_{n-1,2}, \sigma_{n-1,3}, \ldots, \sigma_{n-1, n-1}\right) \subset \mathbb{A}_{k}^{\frac{(n+1)(n-2)}{2}}
$$

é equidimensional com

$$
\operatorname{dim}\left(V_{n-1}\right)=\frac{(n-1)(n-2)}{2}
$$

onde

$$
\sigma_{n-1, j}=\sum_{n-1>t_{1}>t_{2}>\cdots>t_{j-1} \geq 1} X_{n-1, t_{1}} X_{t_{1} t_{2}} \cdots X_{t_{j-2} t_{j-1}} X_{t_{j-1}, n-1} ; j=2,3, \ldots, n-1 .
$$

Mostremos que

$$
V_{n}=V\left(\sigma_{n 2}, \sigma_{n 3}, \sigma_{n 4}, \ldots, \sigma_{n n}\right) \subset \mathbb{A}_{k}^{\frac{(n+2)(n-1)}{2}}
$$

é equidimensional com

$$
\operatorname{dim}\left(V_{n}\right)=\frac{(n+2)(n-1)}{2}-(n-1)=\frac{n(n-1)}{2}
$$

onde

$$
\sigma_{n j}=\sum_{n>t_{1}>t_{2}>\cdots>t_{j-1} \geq 1} X_{n t_{1}} X_{t_{1} t_{2}} \cdots X_{t_{j-2} t_{j-1}} X_{t_{j-1} n} ; j=2,3, \ldots, n .
$$

De fato, como

$$
\sigma_{n n}=X_{n, n-1} X_{n-1, n-2} \cdots X_{32} X_{21} X_{1 n}
$$

então

$$
V_{n}=V\left(\sigma_{n 2}, \sigma_{n 3}, \ldots, \sigma_{n n-1}, X_{n n-1}\right) \cup \bigcup_{t=2}^{n-1} V\left(\sigma_{n 2}, \sigma_{n 3}, \ldots, \sigma_{n n-1}, X_{t t-1}\right) \cup V\left(\sigma_{n 2}, \sigma_{n 3}, \ldots, \sigma_{n n-1}, X_{1 n}\right) .
$$

Para $V\left(\sigma_{n 2}, \sigma_{n 3}, \ldots, \sigma_{n n-1}, X_{1 n}\right) \subset \mathbb{A}_{k}^{\frac{(n+2)(n-1)}{2}}$, como consequência da proposição (2.69) e do corolário (2.67), é suficiente provar que ${ }^{9}$

$$
V_{n}^{(1)}:=V\left(\sigma_{n 2}, \sigma_{n 3}, \ldots, \sigma_{n n-1}, X_{1 n}, X_{21}, X_{31}, X_{41}, \ldots, X_{n 1}\right) \subset \mathbb{A}_{k}^{\frac{(n+2)(n-1)}{2}}
$$

é equidimensional com

$$
\operatorname{dim}\left(V_{n}^{(1)}\right)=\frac{(n+2)(n-1)}{2}-(2 n-2)=\frac{(n-2)(n-1)}{2} .
$$

Para isso, pelas proposições (2.69) e (2.70), é equivalente provar que a variedade

$$
V_{n}^{(2)}:=V\left(\sigma_{n 2}^{\mathbf{X}}, \sigma_{n 3}^{\mathbf{X}}, \ldots, \sigma_{n n-1}^{\mathbf{X}}\right) \subset \mathbb{A}_{k}^{\frac{(n+2)(n-1)}{2}-n}=\mathbb{A}_{k}^{\frac{(n-2)(n+1)}{2}}
$$

\footnotetext{
${ }^{9}$ Observemos que as variáveis $X_{i 1} \operatorname{com} i=2,3, \ldots, n$ não aparecem nos polinômios $\sigma_{n j}^{\mathrm{x}}$ para qualquer $j=2,3, \ldots, n$.
} 
é equidimensional com

$$
\operatorname{dim}\left(V_{n}^{(2)}\right)=\frac{(n-2)(n+1)}{2}-(n-2)=\frac{(n-2)(n-1)}{2},
$$

onde

$$
\mathbf{X}=\left\{X_{1 n}, X_{21}, X_{31}, X_{41}, \ldots, X_{n 1}\right\}
$$

Observemos que ${ }^{10}$

$$
\sigma_{n j}^{\mathbf{X}}=\sum_{n>t_{1}>t_{2}>\cdots>t_{j-1}>1} X_{n t_{1}} X_{t_{1} t_{2}} \cdots X_{t_{j-2} t_{j-1}} X_{t_{j-1} n} \in k\left[I_{n}^{(1)}\right] ; j=2,3, \ldots, n-1
$$

pois $t_{s} \neq 1, \forall s=1,2, \ldots, j-1$ e se considerarmos o isomorfismo de $k$-álgebras

$$
\begin{aligned}
\varphi: k\left[I_{n-1}\right] & \longrightarrow k\left[I_{n}^{(1)}\right] \\
X_{i j} & \longmapsto \varphi\left(X_{i j}\right):=X_{i+1, j+1}
\end{aligned},
$$

temos que para qualquer $j=2,3, \ldots, n-1$

$$
\begin{aligned}
\varphi\left(\sigma_{n-1, j}\right) & =\varphi\left(\sum_{n-1>t_{1}>t_{2}>\cdots>t_{j-1} \geq 1} X_{n-1, t_{1}} X_{t_{1} t_{2}} \cdots X_{t_{j-2} t_{j-1}} X_{t_{j-1}, n-1}\right) \\
& =\sum_{n-1>t_{1}>t_{2}>\cdots>t_{j-1} \geq 1} X_{n, t_{1}+1} X_{t_{1}+1, t_{2}+1} \cdots X_{t_{j-2}+1, t_{j-1}+1} X_{t_{j-1}+1, n} \\
& =\sum_{n>t_{1}>t_{2}>\cdots>t_{j-1}>1} X_{n t_{1}} X_{t_{1}, t_{2}} \cdots X_{t_{j-2}, t_{j-1}} X_{t_{j-1}, n} \\
& =\sigma_{n j}^{\mathbf{X}} .
\end{aligned}
$$

Portanto $V_{n}^{(2)} \cong V_{n-1}$, que por hipótese indutiva é equidimensional com dimensão

$$
\operatorname{dim}\left(V_{n}^{(2)}\right)=\operatorname{dim}\left(V_{n-1}\right)=\frac{(n-1)(n-2)}{2} .
$$

Para $V\left(\sigma_{n 2}, \sigma_{n 3}, \ldots, \sigma_{n n-1}, X_{t-1}\right) \subset \mathbb{A}_{k}^{\frac{(n+2)(n-1)}{2}} \operatorname{com} t \in\{2,3, \cdots, n-1\}$, segue da proposição (2.69) e do corolário (2.67), que é suficiente provar que a variedade ${ }^{11}$

$$
V_{n}^{(1)}:=V\left(\sigma_{n 2}, \sigma_{n 3}, \ldots, \sigma_{n n-1}, X_{t t-1}, X_{t 1}, X_{t 2}, \ldots, X_{t, t-2}, X_{t n}, X_{t+1, t}, X_{t+2, t}, \ldots, X_{n t}\right) \subset \mathbb{A}_{k}^{\frac{(n+2)(n-1)}{2}}
$$

é equidimensional com

$$
\operatorname{dim}\left(V_{n}^{(1)}\right)=\frac{(n+2)(n-1)}{2}-(2 n-2)=\frac{(n-2)(n-1)}{2} .
$$

Para isso, pelas proposições (2.69) e (2.70), é equivalente mostrar que

$$
V_{n}^{(2)}:=V\left(\sigma_{n 2}^{\mathbf{X}}, \sigma_{n 3}^{\mathbf{X}}, \ldots, \sigma_{n n-1}^{\mathbf{X}}\right) \subset \mathbb{A}_{k}^{\frac{(n+2)(n-1)}{2}-n}=\mathbb{A}_{k}^{\frac{(n-2)(n+1)}{2}}
$$

é equidimensional com

$$
\operatorname{dim}\left(V_{n}^{(2)}\right)=\frac{(n-2)(n+1)}{2}-(n-2)=\frac{(n-2)(n-1)}{2},
$$

\footnotetext{
${ }^{10} \sigma_{n j}^{\mathbf{x}} \neq 0, \forall j=2,3, \ldots, n-1$.

${ }^{11}$ Note que, estamos acrescentando a $t$-ésima linha e a $t$-ésima coluna da matriz $I_{n}$.
} 
onde

$$
\mathbf{X}=\left\{X_{t-1}, X_{t 1}, X_{t 2}, \ldots, X_{t, t-2}, X_{t n}, X_{t+1, t}, X_{t+2, t}, \ldots, X_{n t}\right\}
$$

Observemos que ${ }^{12}$

$$
\sigma_{n j}^{\mathbf{X}}=\sum_{\substack{n>t_{1}>t_{2}>\cdots>t_{j-1} \geq 1 \\ t_{s} \neq t}} X_{n t_{1}} X_{t_{1} t_{2}} \cdots X_{t_{j-2} t_{j-1}} X_{t_{j-1} n} ; j=2,3, \ldots, n-1
$$

pois $t_{s} \neq t, \forall s=1,2, \ldots, j-1$ e se considerarmos o isomorfismo de $k$-álgebras ${ }^{13}$

$$
\begin{aligned}
\varphi: k\left[I_{n-1}\right] & \longrightarrow k\left[I_{n}^{(t)}\right] \\
X_{i j} & \longrightarrow \varphi\left(X_{i j}\right):=\left\{\begin{array}{cc}
X_{i j} ; & \text { se } i, j<t \\
X_{i+1, j} ; & \text { se } j<t \leq i \\
X_{i, j+1} ; & \text { se } i<t \leq j \\
X_{i+1, j+1} ; & \text { se } t \leq i, j
\end{array}\right.
\end{aligned}
$$

temos que para qualquer $j=2,3, \ldots, n-1$

$$
\begin{aligned}
\varphi\left(\sigma_{n-1, j}\right) & =\varphi\left(\sum_{n-1>t_{1}>t_{2}>\cdots>t_{j-1} \geq 1} X_{n-1, t_{1}} X_{t_{1} t_{2}} \cdots X_{t_{j-2} t_{j-1}} X_{t_{j-1}, n-1}\right) \\
& =\sum_{\substack{n>t_{1}>t_{2}>\cdots>t_{j-1} \geq 1 \\
t_{s} \neq t}} X_{n t_{1}} X_{t_{1}, t_{2}} \cdots X_{t_{j-2}, t_{j-1}} X_{t_{j-1}, n} \\
& =\sigma_{n j}^{\mathbf{X}} .
\end{aligned}
$$

Portanto $V_{n}^{(2)} \cong V_{n-1}$, que por hipótese indutiva é equidimensional com dimensão

$$
\operatorname{dim}\left(V_{n}^{(2)}\right)=\operatorname{dim}\left(V_{n-1}\right)=\frac{(n-1)(n-2)}{2} .
$$

Para $V\left(\sigma_{n 2}, \sigma_{n 3}, \ldots, \sigma_{n n-1}, X_{n n-1}\right) \subset \mathbb{A}_{k}^{\frac{(n+2)(n-1)}{2}}$, como consequência da proposição (2.69) e do corolário (2.67), é suficiente provar que ${ }^{14}$

$$
V_{n}^{(1)}:=V\left(\sigma_{n 2}, \sigma_{n 3}, \ldots, \sigma_{n n-1}, X_{n n-1}, X_{n-1,1}, X_{n-1,2}, \ldots, X_{n-1, n-2}, X_{n-1, n}\right) \subset \mathbb{A}_{k}^{\frac{(n+2)(n-1)}{2}}
$$

é equidimensional com

$$
\operatorname{dim}\left(V_{n}^{(1)}\right)=\frac{(n+2)(n-1)}{2}-(2 n-2)=\frac{(n-1)(n-2)}{2} .
$$

Pelas proposições (2.69) e (2.70), é equivalente a mostrar que

$$
V_{n}^{(2)}:=V\left(\sigma_{n 2}^{\mathbf{X}}, \sigma_{n 3}^{\mathbf{X}}, \ldots, \sigma_{n n-1}^{\mathbf{X}}\right) \subset \mathbb{A}_{k}^{\frac{(n+2)(n-1)}{2}-n}=\mathbb{A}_{k}^{\frac{(n-2)(n+1)}{2}},
$$

${ }^{12} \sigma_{n j}^{\mathbf{X}} \neq 0$, pois se $j \leq t$, então $\sigma_{n j}^{\mathbf{X}}$ tem o monômio

$$
X_{n, j-1} X_{j-1, j-2} X_{j-2, j-3} \cdots X_{32} X_{21} X_{1 n}
$$

e se $j>t$, então $\sigma_{n j}^{\mathrm{X}}$ tem o monômio

$$
X_{n, n-1} X_{n-1, n-2} \cdots X_{n-(j-t+1), n-(j-t)} X_{n-(j-t), t-1} X_{t-1, t-2} X_{t-2, t-3} \cdots X_{32} X_{21} X_{1 n}
$$

${ }^{13}$ Aqui estamos usando a correspondência natural de tirar da matriz $I_{n}$ a $t$-ésima linha e a $t$-ésima coluna.

${ }^{14}$ Aqui não podemos usar a mesma técnica dos dois casos anteriores, porque se acrescentarmos a $n$-ésima coluna ou a $n$-ésima linha estaríamos eliminando todas as equações, i.e., $\sigma_{n j}^{\mathbf{x}}=0$ para qualquer $j=2,3, \ldots, n-1$ e portanto a sequência $\sigma_{n 2}^{\mathrm{X}}, \sigma_{n 3}^{\mathrm{X}}, \sigma_{n 4}^{\mathrm{X}}, \ldots, \sigma_{n, n-1}^{\mathrm{X}}$ não seria uma sequência regular. Neste caso, só acrescentamos a linha $n-1$ de $I_{n}$. 
onde $\mathbf{X}=\left\{X_{n, n-1}, X_{n-1,1}, X_{n-1,2}, \ldots, X_{n-1, n-2}, X_{n-1, n}\right\}$ é equidimensional com

$$
\operatorname{dim}\left(V_{n}^{(2)}\right)=\frac{(n-2)(n+1)}{2}-(n-2)=\frac{(n-2)(n-1)}{2} .
$$

Não é difícil notar que para $j=2,3, \ldots, n-1$

$$
\sigma_{n j}^{\mathbf{X}}=\sum_{n-1>t_{1}>t_{2}>\cdots>t_{j-1} \geq 1} X_{n t_{1}} X_{t_{1} t_{2}} \cdots X_{t_{j-2} t_{j-1}} X_{t_{j-1} n}
$$

Agora, consideremos o isomomorfismo de $k$-álgebras ${ }^{15}$

$$
\begin{aligned}
\varphi: k\left[I_{n-1}\right] & \longrightarrow k\left[I_{n}^{(n-1)}\right] \\
X_{i j} & \longrightarrow \varphi\left(X_{i j}\right):=\left\{\begin{array}{cc}
X_{i j} ; & \text { se } i, j \neq n-1 \\
X_{n j} ; & \text { se } i=n-1 \\
X_{i n} ; & \text { se } j=n-1
\end{array} .\right.
\end{aligned}
$$

Assim, para qualquer $j=2,3, \ldots, n-1$

$$
\begin{aligned}
\varphi\left(\sigma_{n-1, j}\right) & =\varphi\left(\sum_{n-1>t_{1}>t_{2}>\cdots>t_{j-1} \geq 1} X_{n-1, t_{1}} X_{t_{1} t_{2}} \cdots X_{t_{j-2} t_{j-1}} X_{t_{j-1}, n-1}\right) \\
& =\sum_{n-1>t_{1}>t_{2}>\cdots>t_{j-1} \geq 1} X_{n t_{1}} X_{t_{1}, t_{2}} \cdots X_{t_{j-2}, t_{j-1}} X_{t_{j-1}, n} \\
& =\sigma_{n j}^{\mathbf{X}} .
\end{aligned}
$$

Portanto $V_{n}^{(2)} \cong V_{n-1}$. Assim, da hipótese indutiva temos que a variedade $V_{n}^{(2)}$ é equidimensional com

$$
\operatorname{dim}\left(V_{n}^{(2)}\right)=\operatorname{dim}\left(V_{n-1}\right)=\frac{(n-1)(n-2)}{2} .
$$

Finalmente, dos três casos concluimos que a variedade $V_{n}$ é equidimensional com

$$
\operatorname{dim}\left(V_{n}\right)=\frac{n(n-1)}{2}
$$

\subsubsection{Teorema de Kostant-Wallach}

Definição 3.20. Diremos que a variedade algébrica $V\left(f_{1}, f_{2}, \ldots, f_{t}\right) \subset \mathbb{A}_{k}^{n}$ é uma variedade regular, se os polinômios $f_{1}, f_{2}, \ldots, f_{t} \in k\left[X_{1}, X_{2}, \ldots, X_{n}\right]$ são todos variáveis, ou seja, para qualquer $i$

$$
f_{i}\left(X_{1}, X_{2}, \ldots, X_{n}\right)=X_{j} \text {, paraalgum } j \text {. }
$$

Sem perda de generalidade assumiremos que

$$
f_{i} \neq f_{j}, \forall i \neq j .
$$

Exemplo 3.21. Claramente as seguintes variedades são regulares:

$$
\begin{gathered}
V(X) \subset \mathbb{A}_{k}^{2}, \\
V\left(X_{s_{1}}, X_{s_{2}}, \ldots, X_{s_{t}}\right) \subset \mathbb{A}_{k}^{n}, \text { onde } n \geq t .
\end{gathered}
$$

\footnotetext{
${ }^{15}$ Aqui estamos só transladando a $(n-1)$-ésima coluna e a $(n-1)$-ésima linha da matriz $I_{n-1}$ para a $n$-ésima coluna e a $n$-ésima linha da matriz $I_{n}^{(n-1)}$, já que em $\sigma_{n j}^{\mathbf{X}}$ não aparece nenhuma variável do tipo $X_{n-1, i}$ ou $X_{i, n-1}$.
} 
Observação 3.22. Sejam $V\left(X_{i_{1}}, X_{i_{2}}, \ldots, X_{i_{r}}\right)$ e $V\left(X_{j_{1}}, X_{j_{2}}, \ldots, X_{j_{r}}\right)$ duas subvariedades regulares de uma variedade $V \in \mathbb{A}^{n}$, então existe uma permutação $\sigma \in S_{n}$ tal que

$$
X_{\sigma\left(i_{t}\right)}=X_{t}, \forall t=1,2, \ldots, r .
$$

Portanto, temos o isomorfismo de k-álgebras

$$
\begin{aligned}
\varphi_{\sigma}: k\left[X_{1}, X_{2}, \ldots, X_{n}\right] & \longrightarrow k\left[X_{1}, X_{2}, \ldots, X_{n}\right] \\
X_{i} & \longmapsto \varphi_{\sigma}\left(X_{i}\right):=X_{\sigma(i)}
\end{aligned},
$$

o qual induz o isomorfismo de variedades

$$
\begin{aligned}
f: V\left(X_{i_{1}}, X_{i_{2}}, \ldots, X_{i_{r}}\right) & \longrightarrow V\left(X_{j_{1}}, X_{j_{2}}, \ldots, X_{j_{r}}\right) \\
\left(a_{1}, a_{2}, \ldots, a_{n}\right) & \longrightarrow f\left(a_{1}, a_{2}, \ldots, a_{n}\right):=\left(a_{\sigma(1)}, a_{\sigma(2)}, \ldots, a_{\sigma(n)}\right) .
\end{aligned}
$$

Observemos que $\varphi_{\sigma}\left(I\left(V\left(X_{j_{1}}, X_{j_{2}}, \ldots, X_{j_{r}}\right)\right)\right)=I\left(V\left(X_{i_{1}}, X_{i_{2}}, \ldots, X_{i_{r}}\right)\right)$.

“؟” Sejam $p \in \varphi_{\sigma}\left(I\left(V\left(X_{j_{1}}, X_{j_{2}}, \ldots, X_{j_{r}}\right)\right)\right)$ e $a=\left(a_{1}, a_{2}, \ldots, a_{n}\right) \in V\left(X_{i_{1}}, X_{i_{2}}, \ldots, X_{i_{r}}\right)$, segue-se que existe $q \in I\left(V\left(X_{j_{1}}, X_{j_{2}}, \ldots, X_{j_{r}}\right)\right)$ tal que $\varphi_{\sigma}(q)=p$, e como $f(a) \in V\left(X_{j_{1}}, X_{j_{2}}, \ldots, X_{j_{r}}\right)$, então

$$
p(a)=\varphi_{\sigma}(q)(a)=q\left(X_{\sigma(1)}, X_{\sigma(2)}, \ldots, X_{\sigma(n)}\right)(a)=q\left(a_{\sigma(1)}, a_{\sigma(2)}, \ldots, a_{\sigma(n)}\right)=q(f(a))=0 .
$$

“Р” Seja $p \in I\left(V\left(X_{i_{1}}, X_{i_{2}}, \ldots, X_{i_{r}}\right)\right)$, vejamos que $q=\varphi_{\sigma}^{-1}(p) \in I\left(V\left(X_{j_{1}}, X_{j_{2}}, \ldots, X_{j_{r}}\right)\right)$, para isto consideremos $a \in V\left(X_{j_{1}}, X_{j_{2}}, \ldots, X_{j_{r}}\right)$, logo $f^{-1}(a) \in V\left(X_{i_{1}}, X_{i_{2}}, \ldots, X_{i_{r}}\right)$ e portanto

$$
\begin{gathered}
q(a)=\varphi^{-1}(p)(a)=p\left(X_{\sigma^{-1}(1)}, X_{\sigma^{-1}(2)}, \ldots, X_{\sigma^{-1}(n)}\right)(a)=p\left(a_{\sigma^{-1}(1)}, a_{\sigma^{-1}(2)}, \ldots, a_{\sigma^{-1}(n)}\right) \\
q(a)=p\left(f^{-1}(a)\right)=0 .
\end{gathered}
$$

Agora, como $\varphi_{\sigma}\left(I\left(V\left(X_{j_{1}}, X_{j_{2}}, \ldots, X_{j_{r}}\right)\right)\right)=I\left(V\left(X_{i_{1}}, X_{i_{2}}, \ldots, X_{i_{r}}\right)\right)$, então $I\left(V\left(X_{j_{1}}, X_{j_{2}}, \ldots, X_{j_{r}}\right)\right)$ é um ideal primo minimal se, e somente se, $I\left(V\left(X_{i_{1}}, X_{i_{2}}, \ldots, X_{i_{r}}\right)\right)$ é um ideal primo minimal. Equivalentemente, $V\left(X_{j_{1}}, X_{j_{2}}, \ldots, X_{j_{r}}\right)$ é uma componente irredutível se, e somente se, $V\left(X_{i_{1}}, X_{i_{2}}, \ldots, X_{i_{r}}\right)$ é uma componente irredutível.

O Teorema de Kostant-Wallach em [Kostant e Wallach (2006a)] e [Kostant e Wallach (2006b)] afirma que todas as componentes regulares são equidimensionais com dimensão $\frac{n(n-1)}{2}$. Mas, como consequência da versão fraca temos o seguinte corolário:

Corolário 3.23. Todas as componentes regulares da variedade de Gelfand-Tsetlin são isomorfas. Em particular, são isomorfas à componente irredutível

$$
V_{\leq}:=V\left(\left\{X_{i j}: 1 \leq i \leq j \leq n\right\}\right) .
$$

\section{Demonstração:}

Consideremos uma componente regular da variedade de Gelfand-Tsetlin da forma

$$
V\left(f_{1}, f_{2}, \ldots, f_{d(n)}\right) \subset \mathbb{A}_{k}^{n^{2}}
$$

vejamos que

$$
d(n)=\frac{n(n+1)}{2} .
$$

Claramente para qualquer $i \in\{1,2, \ldots, n\}$, existe $t \in\{1,2, \ldots, d(n)\}$ tal que

$$
f_{t}=X_{i i} \text {, }
$$


caso contrário existiria $A \in V\left(f_{1}, f_{2}, \ldots, f_{d(n)}\right) \subset \mathbb{A}_{k}^{n^{2}}$ tal que

$$
\bar{\gamma}_{i 1}(A)=A_{i i} \neq 0 \text {, }
$$

por exemplo a matriz elementar ${ }^{16} E_{i i}$.

Também temos que, para quaisquer $i, j \in\{1,2, \ldots, n\}$ com $i \neq j$, existe $t \in\{1,2, \ldots, d(n)\}$ tal que

$$
f_{t}=X_{i j} \text { ou } f_{t}=X_{j i}
$$

caso contrário, se $i>j$ existiria $A \in V\left(f_{1}, f_{2}, \ldots, f_{d(n)}\right) \subset \mathbb{A}_{k}^{n^{2}}$ tal que

$$
\bar{\gamma}_{i 2}(A)=\sum_{t=1}^{i-1} A_{i t} A_{t i}=\sum_{t=1}^{j-1} A_{i t} A_{t i}+\underbrace{A_{i j} A_{j i}}_{\neq 0}+\sum_{t=j+1}^{i-1} A_{i t} A_{t i} \neq 0,
$$

por exemplo $A=E_{i j}+E_{j i}$. De maneira similar, se $i<j$ então

$$
d(n) \geq \frac{n(n+1)}{2}
$$

Agora reparemos que se $d(n)>\frac{n(n+1)}{2}$, então existem $i, j \in\{1,2, \ldots, N\} \operatorname{com} i>j$ tais que

$$
X_{i j}, X_{j i} \in\left\{f_{1}, f_{2}, \ldots, f_{d(N)}\right\} .
$$

Consideremos um conjunto $S \subset\left\{X_{i j}: i, j=1,2, \ldots, N\right\}$ com as seguintes condições:

1. $S$ tem $d(n)$ elementos.

2. $\left\{X_{i j}: 1 \leq i \leq j \leq n\right\} \subset S$.

Claramente $V(S)$ é uma subvariedade da variedade de Gelfand-Tsetlin e da observação anterior (3.22)

$$
V\left(f_{1}, f_{2}, \ldots, f_{d(n)}\right) \cong V(S) .
$$

Notemos que $V(S)$ é irredutível, mas, não é uma componente, já que não é uma subvariedade maximal

$$
V(S) \subsetneq V_{\leq}
$$

o qual contradiz a hipótese de que $V\left(f_{1}, f_{2}, \ldots, f_{d(n)}\right)$ seja uma componente regular.

Assim temos que se $V\left(f_{1}, f_{2}, \ldots, f_{d(n)}\right)$ é uma componente regular, então

$$
d(n)=\frac{n(n+1)}{2}
$$

e portanto,

$$
\operatorname{dim}\left(V\left(f_{1}, f_{2}, \ldots, f_{d(n)}\right)\right)=n^{2}-\frac{n(n+1)}{2}=\frac{n(n-1)}{2} .
$$

Além disso, da observação (3.22)

$$
V\left(f_{1}, f_{2}, \ldots, f_{d(n)}\right) \cong V_{\leq}
$$

\footnotetext{
${ }^{16}$ Aqui estamos visualizando os elementos no espaço afim $\mathbb{A}_{k}^{n^{2}}$ como matrizes quadradas $n \times n$ e, por matriz elementar $E_{i j}$ entenderemos a matriz com 1 na entrada $(i, j)$ e zero no resto.
} 


\section{Capítulo 4}

\section{Equidimensionalidade das fibras da aplicação de Kostant-Wallach e da aplicação parcial de Kostant-Wallach}

Começaremos este capítulo definindo PBW álgebras e álgebras filtradas especiais, além de mostrar alguns resultados sobre estas álgebras. Seguiremos com a aplicação de Kostant-Wallach

$$
\varphi: M_{n}(\mathbb{C}) \longrightarrow \mathbb{C}^{\frac{n(n+1)}{2}},
$$

a aplicação de $k$-parcial de Kostant-Wallach

$$
\varphi_{k}: M_{n}(\mathbb{C}) \longrightarrow \mathbb{C}^{n-k+1} \times \cdots \times \mathbb{C}^{n-1} \times \mathbb{C}^{n}
$$

e a relação das fibras em zero $\varphi^{-1}(0)$ e $\varphi_{k}^{-1}(0)$ com a variedade de Gelfand-Tsetlin. Encerraremos este capítulo provando o seguinte teorema:

\section{Teorema:}

Para todo $k=1,2, \ldots, n$ e todo $\beta \in \mathbb{C}^{n-k+1} \times \cdots \times \mathbb{C}^{n-1} \times \mathbb{C}^{n}$ a variedade

$$
\widetilde{V}_{\beta}^{k}=V\left(\left\{\overline{\gamma_{i j}}-\beta_{i j}: n-k+1 \leq i \leq n \text { e } 1 \leq j \leq i\right\}\right) \subset \mathbb{C}^{n^{2}}
$$

é equidimensional com dimensão

$$
\operatorname{dim}\left(\widetilde{V}_{\beta}^{k}\right)=n^{2}-n k+\frac{k(k-1)}{2} .
$$

O qual, claramente é uma extensão do Teorema de Ovsienko. Com este teorema obtemos como corolários a equidimensionalidade das fibras $\varphi^{-1}(\alpha)$ e $\varphi_{k}^{-1}(\alpha)$.

Este teorema e seus corolários, são os resultados principais deste capítulo.

\subsection{PBW álgebras e álgebras filtradas especiais}

Definição 4.1. Seja U uma álgebra associativa filtrada sobre $k$, isto é, possui uma filtração crescente $\left\{U_{i}\right\}_{i \geq 0}$, em que

$$
\begin{gathered}
U_{0}=k, \\
U_{i} U_{j} \subseteq U_{i+j}
\end{gathered}
$$


$e$

$$
U=\bigcup_{i=0}^{\infty} U_{i}
$$

Para $u \in U_{i} / U_{i-1}$ denotemos o grau de $u$ por $\operatorname{deg}(u)=i$ e por convenção consideraremos $U_{-1}=\{0\}$.

Da observação (2.7), a álgebra graduada associada a $U$ é

$$
\bar{U}=\operatorname{gr}(U)=\bigoplus_{i=0}^{\infty} U_{i} / U_{i-1}
$$

Para $u \in U$ denote por $\bar{u}$ sua imagem em $\bar{U}$.

Uma álgebra U é chamada de PBW álgebra, se qualquer elemento de U pode ser escrito de forma única como uma combinação linear de monômios ordenados em alguns geradores fixados de $U$. Se $U$ é uma PBW álgebra tal que gr $(U)$ é uma álgebra de polinômios, então $U$ será chamada de filtrada especial ou PBW álgebra especial.

Observação 4.2. Note que pelo Teorema de Poincaré-Birkhoff-Witt (2.28), a álgebra envolvente universal de qualquer álgebra de Lie de dimensão finita é uma álgebra filtrada especial.

Consideremos a seguinte aplicação

$$
(,)_{U}: U^{t} \times U^{t} \longrightarrow U
$$

tal que, para $\mathbf{u}=\left(u_{1}, u_{2}, \ldots, u_{t}\right)$ e $\mathbf{v}=\left(v_{1}, v_{2}, \ldots, v_{t}\right)$ em $U^{t}$ está dada por

$$
(\mathbf{u}, \mathbf{v})_{U}=\sum_{i=1}^{t} u_{i} v_{i}
$$

Lema 4.3. Sejam $U$ uma álgebra filtrada especial, $g_{1}, g_{2}, \ldots, g_{t} \in U$ elementos mutuamente comutativos tais que, $\bar{g}_{1}, \bar{g}_{2}, \ldots, \bar{g}_{t}$ é uma sequência regular em gr $(U) e I \subseteq U$ o ideal à esquerda de $U$ gerado por $g_{1}, g_{2}, \ldots, g_{t}$. Então, qualquer $f \in I$ pode ser escrito na forma

$$
f=\sum_{j=1}^{t} f_{j} g_{j},
$$

para alguns $f_{j} \in U, j=1,2, \ldots, t$, tais que

$$
\operatorname{deg}(f)=\max _{1 \leq j \leq t} \operatorname{deg}\left(f_{j} g_{j}\right) .
$$

Demonstração: Seja $f \in I$ e consideremos $f=f_{1} g_{1}+f_{2} g_{2}+\cdots+f_{t} g_{t}$ com o menor possível

$$
d=\max _{1 \leq i \leq t} \operatorname{deg}\left(f_{i} g_{i}\right)
$$

Podemos assumir que $d=\operatorname{deg}\left(f_{i} g_{i}\right)$ se, e somente se, $i=1,2, \ldots, r$ para algum $r \leq t$. Consideremos

$$
\begin{aligned}
& \mathbf{f}=\left(f_{1}, f_{2}, \ldots, f_{t}\right), \\
& \mathbf{g}=\left(g_{1}, g_{2}, \ldots, g_{t}\right)
\end{aligned}
$$

e para um vetor $\mathbf{s}=\left(s_{1}, s_{2}, \ldots, s_{t}\right) \in U^{t}$ denotemos $\overline{\mathbf{s}}=\left(\bar{s}_{1}, \bar{s}_{2}, \ldots, \bar{s}_{r}, 0,0, \ldots, 0\right) \in \bar{U}^{t}$.

Se $d>\operatorname{deg}(f)$, do fato que $(\mathbf{f}, \mathbf{g})_{U}=f$ segue que $(\overline{\mathbf{f}}, \overline{\mathbf{g}})_{\bar{U}}=0$ e da resolução de Koszul (prop. (2.68)) temos $\eta\left(\bar{f}_{1}, \bar{f}_{2}, \ldots, \bar{f}_{r}\right)=0$, isto implica que

$$
\left(\bar{f}_{1}, \bar{f}_{2}, \ldots, \bar{f}_{r}\right) \in \operatorname{ker}(\eta) .
$$

Dado que $U$ é uma álgebra filtrada especial, então pelo corolário (2.67) as sequências $\bar{g}_{1}, \bar{g}_{2}, \ldots, \bar{g}_{r}$ são 
também regulares em $\bar{U}=\operatorname{gr}(U)$. Novamente, da resolução de Koszul (prop. (2.68)) temos

$$
\left(\bar{f}_{1}, \bar{f}_{2}, \ldots, \bar{f}_{r}\right) \in \partial\left(\bigwedge^{2} \bar{U}^{r}\right)
$$

portanto, existem $g_{i j} \in U$ com $1 \leq i<j \leq r$ tais que

$$
\overline{\mathbf{f}}=\sum_{1 \leq i<j \leq r} \bar{g}_{i j}\left(\bar{g}_{i} \mathbf{e}_{j}-\bar{g}_{j} \mathbf{e}_{i}\right)
$$

em que $\mathbf{e}_{1}, \mathbf{e}_{2}, \ldots, \mathbf{e}_{r}$ é a base canônica de $\bar{U}^{r}$.

Agora, consideremos

$$
\mathbf{k}=\sum_{1 \leq i<j \leq r} g_{i j}\left(g_{i} \mathbf{e}_{j}-g_{j} \mathbf{e}_{i}\right) \in U^{t},
$$

$\operatorname{logo} \overline{\mathbf{f}}=\overline{\mathbf{k}}$, assim para $\mathbf{h}=\left(h_{1}, h_{2}, \ldots, h_{t}\right)=\mathbf{f}-\mathbf{k}$ tem-se que

$$
\operatorname{deg}\left(h_{i}\right) \leq \operatorname{deg}\left(f_{i}\right) \text { para cada } i=1,2, \ldots, t
$$

$\mathrm{e}$

$$
\operatorname{deg}\left(h_{i}\right)<\operatorname{deg}\left(f_{i}\right) \text { para cada } i=1,2, \ldots, r .
$$

Note que $\left(g_{i} \mathbf{e}_{j}-g_{j} \mathbf{e}_{i}, \mathbf{g}\right)_{U}=g_{i} g_{j}-g_{j} g_{i}=0, \log 0$

$$
(\mathbf{k}, \mathbf{g})_{U}=0 .
$$

Então

$$
\sum_{i=1}^{t} h_{i} g_{i}=(\mathbf{h}, \mathbf{g})_{U}=(\mathbf{f}-\mathbf{k}, \mathbf{g})_{U}=(\mathbf{f}, \mathbf{g})_{U}=f
$$

Dado que

$$
\max _{1 \leq i \leq t} \operatorname{deg}\left(h_{i} g_{i}\right)<\max _{1 \leq i \leq t} \operatorname{deg}\left(f_{i} g_{i}\right)
$$

temos que isso contradiz a minimalidade de $d$, e portanto obtemos que

$$
d=\operatorname{deg}(f) .
$$

Observação 4.4. Antes da seguinte proposição, note-se que se U é uma álgebra filtrada especial, a graduação sobre $\bar{U}=\operatorname{gr}(U)$ em geral não coincide com a graduação standard da álgebra de polinômios. Em particular, se $d_{1}, d_{2}, \ldots, d_{n}$ são inteiros positivos então $\Lambda=\bar{\Lambda}=\mathbb{K}\left[X_{1}, X_{2}, \ldots, X_{n}\right]$, munida com uma graduação dada por deg $\left(X_{i}\right)=d_{i}$, é uma álgebra filtrada especial com sua correspondente filtração. Quando todos os $d_{i}=1$, claramente obtemos a graduação standard sobre $\Lambda$.

Proposição 4.5. Sejam $\Lambda=k\left[X_{1}, X_{2}, \ldots, X_{n}\right]$ e $g_{1}, g_{2}, \ldots, g_{t} \in \Lambda$ tais que a sequência $\bar{g}_{1}, \bar{g}_{2}, \ldots, \bar{g}_{t}$ é regular em $\Lambda=\operatorname{gr}(\Lambda)$. Então, a sequência $g_{1}, g_{2}, \ldots, g_{t}$ é também regular em $\Lambda$.

Demonstração: Suponha que a sequência $g_{1}, g_{2}, \ldots, g_{t}$ não é regular em $\Lambda$, da definição de sequência regular (2.64), existe $i \in\{1,2, \ldots, t\}$ tal que $g_{i}$ é um divisor de zero ou um elemento inversível em $\Lambda /\left(g_{1}, g_{2}, \ldots, g_{i-1}\right)$.

Se $g_{i}$ é um divisor de zero em $\Lambda /\left(g_{1}, g_{2}, \ldots, g_{i-1}\right)$, então existe $f \in \Lambda$ tal que $f \notin\left(g_{1}, g_{2}, \ldots, g_{i-1}\right)$ e $f g_{i} \in\left(g_{1}, g_{2}, \ldots, g_{i-1}\right)$, isto implica que

$$
f g_{i}=\sum_{j=1}^{i-1} f_{j} g_{j}, \text { para alguns } f_{j} \in \Lambda .
$$


Consideremos $f$ com grau o menor possível, pelo lema (4.3)

$$
\operatorname{deg}\left(f g_{i}\right)=\max _{1 \leq j \leq i-1} \operatorname{deg}\left(f_{j} g_{j}\right)
$$

assim $\bar{f}_{\bar{g}}$ é zero em $\Lambda /\left(\bar{g}_{1}, \bar{g}_{2}, \ldots, \bar{g}_{i-1}\right)$. Como a sequência $\bar{g}_{1}, \bar{g}_{2}, \ldots, \bar{g}_{t}$ é regular, então da definição de sequência regular (2.64), tem-se que $\bar{g}_{1}, \bar{g}_{2}, \ldots, \bar{g}_{i-1}$ é também uma sequência regular. Portanto,

$$
\bar{f}=0 \text { em } \Lambda /\left(\bar{g}_{1}, \bar{g}_{2}, \ldots, \bar{g}_{i-1}\right),
$$

disso

$$
\bar{f}=\sum_{j=1}^{i-1} \bar{h}_{j} \bar{g}_{j} \text {, para alguns } h_{j} \in \Lambda \text {. }
$$

Como consequência

$$
f^{\prime}=f-\sum_{j=1}^{i-1} h_{j} g_{j}
$$

tem um grau menor que $f$. Por outro lado, como $f \notin\left(g_{1}, g_{2}, \ldots, g_{i-1}\right)$ então $f^{\prime} \notin\left(g_{1}, g_{2}, \ldots, g_{i-1}\right)$ e além disso $f^{\prime} g_{i} \in\left(g_{1}, g_{2}, \ldots, g_{i-1}\right)$. Isso contradiz a minimalidade de $f$ e portanto $g_{i}$ não é um divisor de zero em $\Lambda /\left(g_{1}, g_{2}, \ldots, g_{i-1}\right)$. O caso quando a imagem de $g_{i}$ em $\Lambda /\left(g_{1}, g_{2}, \ldots, g_{i-1}\right)$ seja inversível é análogo.

\subsection{Aplicação de Kostant-Wallach e aplicação parcial de Kostant-Wallach}

Primeiro fixaremos a notação. Seja $n \in \mathbb{Z}_{>0}$. Para cada matriz

$$
X=\left(\begin{array}{cccc}
X_{11} & X_{12} & \cdots & X_{1 n} \\
X_{21} & X_{22} & \cdots & X_{2 n} \\
\vdots & \vdots & \ddots & \vdots \\
X_{n 1} & X_{n 2} & \cdots & X_{n n}
\end{array}\right) \in M_{n}(\mathbb{C}) .
$$

Denotemos:

1. A $i$-ésima submatriz principal de $X \operatorname{com} i=1,2, \ldots, n$ por

$$
X_{i}:=\left(\begin{array}{cccc}
X_{11} & X_{12} & \cdots & X_{1 i} \\
X_{21} & X_{22} & \cdots & X_{2 i} \\
\vdots & \vdots & \ddots & \vdots \\
X_{i 1} & X_{i 2} & \cdots & X_{i i}
\end{array}\right) \in M_{i}(\mathbb{C})
$$

2. O coeficiente de $t^{i-j}$ do polinômio carácteristico de $X_{i} \operatorname{com} i=1,2, \ldots, n$ e $j=1,2, \ldots, i$ por

$$
\chi_{i j}(X)
$$

i.e., o polinômio característico da $i$-ésima submatriz principal $X_{i}$ é

$$
t^{i}+\chi_{i 1}(X) t^{i-1}+\chi_{i 2}(X) t^{i-2}+\cdots+\chi_{i i-2}(X) t^{2}+\chi_{i i-1}(X) t^{1}+\chi_{i i}(X) t^{0} .
$$

3. E por $\chi_{i}(X)$ o vetor

$$
\chi_{i}(X):=\left(\chi_{i 1}(X), \chi_{i 2}(X), \ldots, \chi_{i i}(X)\right) \in \mathbb{C}^{i}
$$




\section{Definição 4.6.}

1. A aplicação definida como

$$
\begin{aligned}
\varphi: M_{n}(\mathbb{C}) & \longrightarrow \mathbb{C}^{\frac{n(n+1)}{2}} \\
X & \longmapsto \varphi(X):=\left(\chi_{1}(X), \chi_{2}(X), \ldots, \chi_{n}(X)\right)
\end{aligned}
$$

é conhecida como aplicação de Kostant-Wallach.

2. Para cada $k=1,2, \ldots, n$ definiremos a aplicação $k$-parcial de Kostant-Wallach pelo morfismo

$$
\varphi_{k}:=\pi_{k} \circ \varphi
$$

em que $\pi_{k}: \mathbb{C} \times \mathbb{C}^{2} \times \cdots \mathbb{C}^{n} \longrightarrow \mathbb{C}^{n-k+1} \times \cdots \times \mathbb{C}^{n-1} \times \mathbb{C}^{n}$ é a projeção canônica sobre os últimos $k$ vetores. Ou seja,

$$
\begin{aligned}
\varphi_{k}: M_{n}(\mathbb{C}) & \longrightarrow \mathbb{C}^{n-k+1} \times \cdots \times \mathbb{C}^{n-1} \times \mathbb{C}^{n} \\
X & \longmapsto \varphi_{k}(X):=\left(\chi_{n-k+1}(X), \ldots, \chi_{n-1}(X), \chi_{n}(X)\right) .
\end{aligned}
$$

Observação 4.7. Claramente $\varphi_{n}=\varphi$.

\section{Definição 4.8.}

1. Para cada $\alpha \in \mathbb{C}^{\frac{n(n+1)}{2}}$, a fibra em $\alpha$ de $\varphi$ é dada por

$$
\varphi^{-1}(\alpha):=\left\{X \in M_{n}(\mathbb{C}) / \varphi(X)=\alpha\right\} \subset \mathbb{C}^{n^{2}},
$$

a qual claramente é uma variedade algébrica em $\mathbb{C}^{n^{2}}$.

2. Similarmente, para $\alpha \in \mathbb{C}^{n-k+1} \times \cdots \times \mathbb{C}^{n-1} \times \mathbb{C}^{n}$ a fibra em $\alpha$ de $\varphi_{k}$ é dada por

$$
\varphi_{k}^{-1}(\alpha):=\left\{X \in M_{n}(\mathbb{C}) / \varphi_{k}(X)=\alpha\right\} \subset \mathbb{C}^{n^{2}},
$$

a qual claramente também é uma variedade algébrica em $\mathbb{C}^{n^{2}}$.

Proposição 4.9 (Teorema de Colarusso-Evens, 2015).

A fibra em $\alpha \in \mathbb{C}^{n-1} \times \mathbb{C}^{n}$ da aplicação 2-parcial de Kostant-Wallach $\varphi_{2}$ é equidimensional com dimensão

$$
\operatorname{dim}\left(\varphi_{2}^{-1}(\alpha)\right)=n^{2}-2 n+1 .
$$

Demonstração: Ver [Colarusso e Evens (2015)].

\subsection{Aplicação de Kostant-Wallach vs Variedades de Gelfand-Tseltin}

Podemos visualizar as fibras em zero da aplicação de Kostant-Wallach e das aplicações parciais de Kostant-Wallach como variedades de Gelfand-Tsetlin da seguinte forma:

Definição 4.10. Para $k=1,2, \ldots, n$ e $\beta \in \mathbb{C}^{n-k+1} \times \cdots \times \mathbb{C}^{n-1} \times \mathbb{C}^{n}$, definiremos a variedade $k$-parcial de Gelfand-Tsetlin em $\beta$ como a variedade algébrica

$$
\widetilde{V}_{\beta}^{k}=V\left(\left\{\bar{\gamma}_{i j}-\beta_{i j}: n-k+1 \leq i \leq n \text { e } 1 \leq j \leq i\right\}\right) \subset \mathbb{C}^{n^{2}} .
$$

Observação 4.11. Claramente

$$
\widetilde{V}_{0}^{n}=V\left(\left\{\bar{\gamma}_{i j}: 1 \leq j \leq i \leq n\right\}\right)
$$

é a variedade de Gelfand-Tsetlin para $\mathfrak{g l}_{n}$. 
Proposição 4.12. Para cada $i=1,2, \ldots, n$

$$
V\left(\chi_{i 1}, \chi_{i 2}, \ldots, \chi_{i i}\right)=V\left(\bar{\gamma}_{i 1}, \bar{\gamma}_{i 2}, \ldots, \bar{\gamma}_{i i}\right) .
$$

Demonstração: Ver [Želobenko (1973)] página 170 ou [Ovsienko (2003)].

A relação entre a aplicação de Kostant-Wallach e a variedade de Gelfand-Tseltin está determinada pelo seguinte corolário.

Corolário 4.13. Para todo $k=1,2, \ldots, n$, a variedade $k$-parcial de Gelfand-Tsetlin em zero coincide com a fibra em zero da aplicação $k$-parcial de Kostant-Wallach, i.e.,

$$
\widetilde{V}_{0}^{k}=\varphi_{k}^{-1}(0) \text {. }
$$

Demonstração: Dado que

$$
\varphi_{k}^{-1}(0)=\left\{X \in M_{n}(\mathbb{C}) / \varphi_{k}(X)=0\right\}
$$

$\mathrm{e}$

$$
\begin{aligned}
\varphi_{k}: M_{n}(\mathbb{C}) & \longrightarrow \mathbb{C}^{n-k+1} \times \cdots \times \mathbb{C}^{n-1} \times \mathbb{C}^{n} \\
X & \longrightarrow \varphi(X):=\left(\chi_{n-k+1}(X), \ldots, \chi_{n-1}(X), \chi_{n}(X)\right),
\end{aligned}
$$

então

$$
\begin{aligned}
\varphi_{k}^{-1}(0) & =V\left(\left\{\chi_{i j}: n-k+1 \leq i \leq n \text { e } 1 \leq j \leq i\right\}\right) \\
& =\bigcap_{i=n-k+1}^{n} V\left(\chi_{i 1}, \chi_{i 2}, \ldots, \chi_{i i}\right) \\
& =\bigcap_{i=n-k+1}^{n} V\left(\bar{\gamma}_{i 1}, \bar{\gamma}_{i 2}, \ldots, \bar{\gamma}_{i i}\right) \\
& =V\left(\left\{\overline{\gamma_{i j}}: n-k+1 \leq i \leq n \text { e } 1 \leq j \leq i\right\}\right) \\
& =\widetilde{V}_{0}^{k} .
\end{aligned}
$$

Observação 4.14. Segue do corolário anterior, que a variedade de Gelfand-Tsetlin coincide com a fibra em zero da aplicação de Kostant-Wallach $\varphi^{-1}(0)$. Como consequência desse fato, a variedade de GelfandTsetlin para $\mathfrak{g l}_{n}(\mathbb{C})$ é exatamente o conjunto das matrizes quadradas $n \times n$ fortemente nilpotentes (em que, uma matriz $X \in M_{n}(\mathbb{C})$ é dita fortemente nilpotente, se todas suas $i$-ésimas submatrizes principais $X_{i}$ são nilpotentes).

\subsection{Uma generalização do Teorema de Colarusso-Evens}

Nesta seção vamos provar uma generalização do Teorema de Colarusso-Evens.

Teorema 4.15. Para todo $k=1,2, \ldots, n$ e todo $\beta \in \mathbb{C}^{n-k+1} \times \cdots \times \mathbb{C}^{n-1} \times \mathbb{C}^{n}$, a variedade $k$-parcial de Gelfand-Tsetlin $\widetilde{V}_{\beta}^{k}$ é equidimensional com dimensão

$$
\operatorname{dim}\left(\widetilde{V}_{\beta}^{k}\right)=n^{2}-n k+\frac{k(k-1)}{2} .
$$

Em particular, para todo $\alpha \in \mathbb{C}^{\frac{n(n+1)}{2}}$ a variedade $n$-parcial de Gelfand-Tsetlin

$$
\widetilde{V}_{\alpha}^{n}=\left\{x \in \mathbb{C}^{n^{2}}: \bar{\gamma}_{i j}(x)=\alpha_{i j}, \forall i=1,2, \ldots, n ; \forall j=1,2, \ldots, i\right\}
$$

é equidimensional com dimensão

$$
\operatorname{dim}\left(\widetilde{V}_{\alpha}^{n}\right)=\frac{n(n-1)}{2}
$$


Demonstração: Consideremos a sequência em $\operatorname{gr}\left(U\left(\mathfrak{g l}_{n}(\mathbb{C})\right)\right.$

$$
\left\{\sigma_{i j}:=\overline{\gamma_{i j}}-\beta_{i j}: 1 \leq j \leq i, n-k+1 \leq i \leq n\right\} .
$$

Notemos que para $1 \leq j \leq i$ e $n-k+1 \leq i \leq n$ tem-se

$$
\overline{\sigma_{i j}}=\overline{\overline{\gamma_{i j}}-\beta_{i j}}=\overline{\overline{\gamma_{i j}}}=\overline{\gamma_{i j}}
$$

$\operatorname{emgr}\left(U\left(\mathfrak{g l}_{n}(\mathbb{C})\right)=\operatorname{gr}\left(\operatorname{gr}\left(U\left(\mathfrak{g l}_{n}(\mathbb{C})\right)\right)\right.\right.$. Assim, a sequência

$$
\left\{\overline{\sigma_{i j}}=\overline{\gamma_{i j}}: 1 \leq j \leq i, n-k+1 \leq i \leq n\right\}
$$

é uma subsequência de

$$
\left\{\overline{\gamma_{i j}}: 1 \leq j \leq i \leq n\right\},
$$

a qual, pelo Teorema de Ovsienko (3.15) e pela proposição (2.69), é regular em gr $\left(U\left(\mathfrak{g l}_{n}(\mathbb{C})\right)\right)$. Logo, do corolário (2.67), tem-se que

$$
\left\{\overline{\sigma_{i j}}=\overline{\gamma_{i j}}: 1 \leq j \leq i, n-k+1 \leq i \leq n\right\}
$$

é uma sequência regular em $\operatorname{gr}\left(U\left(\mathfrak{g l}_{n}(\mathbb{C})\right)=\operatorname{gr}\left(\operatorname{gr}\left(U\left(\mathfrak{g l}_{n}(\mathbb{C})\right)\right)\right.\right.$.

Como consequência da proposição (4.5), temos que

$$
\left\{\sigma_{i j}:=\overline{\gamma_{i j}}-\beta_{i j}: 1 \leq j \leq i, n-k+1 \leq i \leq n\right\}
$$

é uma sequência regular em $\operatorname{gr}\left(U\left(\mathfrak{g l}_{n}(\mathbb{C})\right)\right)$. Portanto, das proposições (2.68) e (2.69),

$$
\widetilde{V}_{\beta}^{k}=V\left(\left\{\overline{\gamma_{i j}}-\beta_{i j}: 1 \leq j \leq i, n-k+1 \leq i \leq n\right\}\right) \subset \mathbb{C}^{n^{2}}
$$

é uma variedade equidimensional com dimensão

$$
\operatorname{dim}\left(\widetilde{V}_{\beta}^{k}\right)=n^{2}-n-(n-1)-(n-2)-\cdots-(n-k+1)=n^{2}-n k+\frac{k(k-1)}{2} .
$$

Agora podemos concluir a equidimensionalidade das fibras da aplicação de Kostant-Wallach e de suas aplicações parciais.

Corolário 4.16. Para cada $k=1,2, \ldots, n$ e cada $\beta \in \mathbb{C}^{n-k+1} \times \cdots \times \mathbb{C}^{n-1} \times \mathbb{C}^{n}$, a fibra $\varphi_{k}^{-1}(\beta)$ da aplicação $k$-parcial de Kostant-Wallach $\varphi_{k}$ é equidimensional com dimensão

$$
\operatorname{dim}\left(\varphi_{k}^{-1}(\beta)\right)=n^{2}-n k+\frac{k(k-1)}{2} .
$$

Demonstração: Note-se que os polinômios $\chi_{i j}$ são todos homogêneos em $\Lambda=\mathbb{C}\left[X_{i j}: 1 \leq i, j \leq n\right]$ e portanto podemos fazer o mesmo raciocinio feito na prova do teorema anterior (4.15).

Consideremos a sequência

$$
\left\{\chi_{i j}-\beta_{i j}: n-k+1 \leq i \leq n \text { e } 1 \leq j \leq i\right\}
$$

e a graduação $\bar{\Lambda}=\Lambda$ de $\Lambda$ dada pelo grau do polinômio (i.e., $\operatorname{deg}\left(X_{i}^{t}\right)=t$ ).

Para $n-k+1 \leq i \leq n$ e $1 \leq j \leq i$ tem-se

$$
\overline{\chi_{i j}-\beta_{i j}}=\overline{\chi_{i j}}=\chi_{i j} .
$$

Segue do corolário (4.13), que

$$
\widetilde{V}_{0}^{k}=\varphi_{k}^{-1}(0)=V\left(\left\{\chi_{i j}: n-k+1 \leq i \leq n \text { e } 1 \leq j \leq i\right\}\right),
$$


a qual pelo teorema (4.15), é equidimensional com dimensão

$$
n^{2}-n k+\frac{k(k-1)}{2}
$$

Logo da proposição (2.69) a sequência

$$
\left\{\overline{\chi_{i j}-\beta_{i j}}=\chi_{i j}: n-k+1 \leq i \leq n \text { e } 1 \leq j \leq i\right\}
$$

é regular em $\bar{\Lambda}$ e da proposição (4.5) podemos concluir que a sequência

$$
\left\{\chi_{i j}-\beta_{i j}: n-k+1 \leq i \leq n \text { e } 1 \leq j \leq i\right\}
$$

é regular em $\Lambda$. Finalmente das proposições (2.68) e (2.69), a variedade

$$
\varphi_{k}^{-1}(\beta)=V\left(\left\{\chi_{i j}-\beta_{i j}: n-k+1 \leq i \leq n \text { e } 1 \leq j \leq i\right\}\right)
$$

é equidimensional com dimensão

$$
\operatorname{dim}\left(\varphi_{k}^{-1}(\beta)\right)=n^{2}-n k+\frac{k(k-1)}{2} .
$$

Corolário 4.17. Para cada $\alpha \in \mathbb{C}^{\frac{n(n+1)}{2}}$, a fibra $\varphi^{-1}(\alpha)$ da aplicação de Kostant-Wallach $\varphi$ é equidimensional com dimensão

$$
\operatorname{dim}\left(\varphi^{-1}(\alpha)\right)=\frac{n(n-1)}{2} .
$$

Demonstração: Segue do fato que $\varphi_{n}=\varphi$. 


\section{Capítulo 5}

\section{Versão fraca $\mathfrak{G}_{1}$ para Yangians $Y_{p}\left(\mathfrak{g l}_{3}\right)$}

Começaremos este capítulo com uma seção onde definiremos Yangians restritos $Y_{p}\left(\mathfrak{g l}_{n}\right)$ de nível $p$ para $\mathfrak{g l}_{n}$, sua subálgebra de Gelfand-Tsetlin $\Gamma$ e sua variedade de Gelfand-Tsetlin $\mathfrak{G}$. Dada a dificuldade na combinatória dos polinômios que determinam $\mathfrak{G}$, na segunda seção apresentamos uns polinômios um pouco mais acessíveis, os quais também determinam a variedade de Gelfand-Tsetlin $\mathfrak{G}$ para $Y_{p}\left(\mathfrak{g l}_{3}\right)$ e aproveitamos tais polinômios para apresentar a decomposição em componentes irredutíves de $\mathfrak{G}$ para Yangians $Y_{1}\left(\mathfrak{g l}_{3}\right)$ e $Y_{2}\left(\mathfrak{g l}_{3}\right)$. Esta decomposição permitirá concluir nesses casos a equidimensionalidade de $\mathfrak{G}$. Na terceira seção, apresentamos uma decomposição de $\mathfrak{G}$ para $Y_{p}\left(\mathfrak{g l}_{n}\right)$ da forma

$$
\mathfrak{G}=\mathfrak{G}_{1} \cup \mathfrak{G}_{2} \cup \cdots \cup \mathfrak{G}_{p+1}
$$

Essa decomposição não é em componentes irredutíveis, pois na mesma seção mostraremos uma decomposição da subvariedade $\mathfrak{G}_{1}$ de $\mathfrak{G}$ para $Y_{p}\left(\mathfrak{g l}_{n}\right)$ na forma

$$
\begin{gathered}
\mathfrak{G}_{1}=\mathbb{W}_{1} \cup \mathbb{W}_{2} \text {, se } p=1,2 \\
\mathfrak{G}_{1}=\mathbb{W}^{p} \cup \mathbb{W}_{1} \cup \mathbb{W}_{2} \text {, se } p \geq 3 .
\end{gathered}
$$

Quando $n=3$, a variedade $\mathfrak{G}_{1}$ será chamada de versão fraca da variedade de Gelfand-Tsetlin $\mathfrak{G}$ para $Y_{p}\left(\mathfrak{g l}_{3}\right)$. Encerramos este capítulo provando a equidimensionalidade de $\mathbb{W}^{p}, \mathbb{W}_{1}$ e $\mathbb{W}_{2}$ e obtendo portanto o resultado principal deste capítulo.

Teorema: Para todo $p$, a variedade $\mathfrak{G}_{1}$ para $Y_{p}\left(\mathfrak{g l}_{3}\right)$ é equidimensional com dimensão

$$
\operatorname{dim} \mathfrak{G}_{1}=3 p .
$$

\subsection{Yangians restritos $Y_{p}\left(\mathfrak{g l}_{n}\right)$ de nível $p$ para $\mathfrak{g l}_{n}$}

Agora vamos introduzir o objeto que queremos estudar, já que tudo o apresentado nos capítulos 3 e 4 foi encontrado, na procura de técnicas e estratégias para nosso objetivo principal, que é Yangians.

Sejam $p$ e $n$ inteiros positivos. O Yangian ${ }^{1} Y_{p}\left(\mathfrak{g l}_{n}\right)$ de nível $p$ para a álgebra de Lie $\mathfrak{g l}_{n}$, pode ser definido como a álgebra associativa com geradores $t_{i j}^{(1)}, t_{i j}^{(2)}, \ldots, t_{i j}^{(p)}, i, j=1,2, \ldots, n$ e com as relações

$$
\left[T_{i j}(u), T_{k l}(v)\right]=\frac{1}{u-v}\left(T_{k j}(u) T_{i l}(v)-T_{k j}(v) T_{i l}(u)\right),
$$

onde $u, v$ são variáveis formais $\mathrm{e}$

$$
T_{i j}(u)=\delta_{i j} u^{p}+\sum_{k=1}^{p} t_{i j}^{(k)} u^{p-k} \in Y_{p}\left(\mathfrak{g l}_{n}\right)[u] .
$$

${ }^{1}$ Escrevemos "O Yangian", porque $Y_{p}\left(\mathfrak{g l}_{n}\right)$ mais do que uma álgebra é um grupo quântico. 
Essas relações são equivalentes à equação

$$
\left[t_{i j}^{(r)}, t_{k l}^{(s)}\right]=\sum_{a=1}^{\min \{r, s\}}\left(t_{k j}^{(a-1)} t_{i l}^{(r+s-a)}-t_{k j}^{(r+s-a)} t_{i l}^{(a-1)}\right),
$$

onde $t_{i j}^{(0)}=\delta_{i j}$ e $t_{i j}^{(r)}=0$ para $r \geq p+1$.

Para mais detalhes sobre Yangians ver [Cherednik (1989)] e [Drinfel'd (1985)].

\section{Observação 5.1.}

1. O Yangian $Y_{1}\left(\mathfrak{g l}_{n}\right)$ de nível 1 coincide com a álgebra envolvente universal $U\left(\mathfrak{g l}_{n}\right)$.

2. Além disso,

$$
\operatorname{deg}\left(t_{i j}^{(k)}\right)=k, \text { para } i, j=1,2, \ldots, n \text { e } k=1,2, \ldots, p
$$

define uma filtração natural sobre $Y_{p}\left(\mathfrak{g l}_{n}\right)$. Denotaremos a correspondente álgebra graduada por $\bar{Y}_{p}\left(\mathfrak{g l}_{n}\right)$ ou por gr $\left(Y_{p}\left(\mathfrak{g l}_{n}\right)\right)$.

A seguinte proposição é a versão análoga do Teorema de Poincaré-Birkhoff-Witt para $Y_{p}\left(\mathfrak{g l}_{n}\right)$, o qual será muito importante para nossa abordagem, pois ele nos permite usar a linguagem e resultados de álgebra comutativa da mesma forma que foi usada no capítulo 3 , na demonstração da versão fraca do Teorema de Ovsienko (3.18).

\section{Proposição 5.2 (PBW para Yangians).}

A álgebra associativa graduada $\bar{Y}_{p}\left(\mathfrak{g l}_{n}\right)=\operatorname{gr}\left(Y_{p}\left(\mathfrak{g l}_{n}\right)\right)$ é uma álgebra de polinômios nas variáveis $\bar{t}_{i j}^{(k)}$, com $i, j=1,2, \ldots$, n e $k=1,2, \ldots, p$.

Demonstração: Ver [Cherednik (1989)] ou teorema 2.1 em [Molev (1997)].

Considere a matriz $T(u)=\left(T_{i j}(u)\right)_{i, j=1}^{n}$ e o elemento em $Y_{p}\left(\mathfrak{g l}_{n}\right)[u]$, chamado de determinante quântico para $Y_{p}\left(\mathfrak{g l}_{n}\right)$, definido por

$$
\text { qdet } T(u)=\sum_{\sigma \in S_{n}} \operatorname{sgn}(\sigma) T_{1 \sigma(1)}(u) T_{2 \sigma(2)}(u-1) \cdots T_{n \sigma(n)}(u-n+1) .
$$

Proposição 5.3 (Cherednik (1989)). Sejam $p$ e $n$ inteiros positivos. O centro $Z\left(Y_{p}\left(\mathfrak{g l}_{n}\right)\right)$ de $Y_{p}\left(\mathfrak{g l}_{n}\right)$ é gerado pelos coeficientes $d_{n i}$, das potências $u^{n p-i}$ do determinante quântico qdet $T(u)$, o qual é um polinômio mônico em u e de grau $n p$, i.e.,

$$
Z\left(Y_{p}\left(\mathfrak{g l}_{n}\right)\right)=\left\langle d_{n 1}, d_{n 2}, \ldots, d_{n n p}\right\rangle,
$$

em que

$$
\text { qdet } T(u)=u^{n p}+d_{n 1} u^{n p-1}+d_{n 2} u^{n p-2}+\cdots+d_{n n p-1} u+d_{n n p}, \quad d_{n i} \in Y_{p}\left(\mathfrak{g l}_{n}\right) .
$$

Demonstração: Ver teorema (1) em [Cherednik (1989)] ou Corolário (4.1) em [Molev (1997)].

Agora, considere a cadeia de subálgebras

$$
Y_{p}\left(\mathfrak{g l}_{1}\right) \subset Y_{p}\left(\mathfrak{g l}_{2}\right) \subset \cdots \subset Y_{p}\left(\mathfrak{g l}_{n-1}\right) \subset Y_{p}\left(\mathfrak{g l}_{n}\right) .
$$

A subálgebra de Gelfand-Tsetlin $\Gamma$ para Yangians $Y_{p}\left(\mathfrak{g l}_{n}\right)$ é a subálgebra de $Y_{p}\left(\mathfrak{g l}_{n}\right)$ gerada pelos centros $Z\left(Y_{p}\left(\mathfrak{g l}_{i}\right)\right)$ de cada subálgebra $Y_{p}\left(\mathfrak{g l}_{i}\right)(i=1,2, \ldots, n)$. Pela proposição (5.3), os coeficientes do determinante quântico

$$
\left\{d_{i j}\right\}_{\substack{i=1,2, \ldots, n \\ j=1,2, \ldots, i p}}
$$

geram a subálgebra $\Gamma$. 
Observação 5.4. Para

$$
F=\sum_{i} f_{i} u^{i} \in Y_{p}\left(\mathfrak{g l}_{n}\right)[u]
$$

denote

$$
\bar{F}:=\sum_{i} \bar{f}_{i} u^{i} \in \bar{Y}_{p}\left(\mathfrak{g l}_{n}\right)[u] .
$$

Também, denote

$$
\begin{aligned}
X_{i j}^{(k)} & :=\bar{t}_{i j}^{(k)}, \\
X_{i j}(u) & :=\bar{T}_{i j}(u),
\end{aligned}
$$

$e$

$$
X(u):=\left(X_{i j}(u)\right)_{i, j=1}^{n}
$$

Portanto, como

$$
\overline{T_{i j}(u-\alpha)}=X_{i j}(u), \quad \forall \alpha \in k,
$$

podemos facilmente ver que

$$
\text { gr qdet } T(u)=\operatorname{det} X(u) \text {. }
$$

Pelo PBW para Yangians (prop. (5.2)), cada $\bar{d}_{i j}$ é um polinômio nas variáveis comutativas $\bar{t}_{i j}^{(k)}$ com $i, j=1,2, \ldots, n$ e $k=1,2, \ldots, p$.

Definição 5.5. A variedade de Gelfand-Tsetlin para $Y_{p}\left(\mathfrak{g l}_{n}\right)$ é a variedade algébrica

$$
\mathfrak{G}:=V\left(\left\{\overline{d_{i j}}\right\}_{\substack{i=1,2, \ldots, n \\ j=1,2, \ldots, i p}}\right) \subset k^{n^{2} p} .
$$

Observação 5.6. Segue do corolário (4.13), que a variedade de Gelfand-Tsetlin $\mathfrak{G}$ para $Y_{1}\left(\mathfrak{g l}_{n}\right)$ coincide com a variedade de Gelfand-Tsetlin para $\mathfrak{g l}_{n}$.

\subsubsection{Alguns polinômios da variedade de Gelfand-Tsetlin}

Dado que

$$
\begin{aligned}
& \operatorname{grqdet} T(u)=\operatorname{det} X(u)=\sum_{\sigma \in S_{n}} \operatorname{sgn}(\sigma) X_{1 \sigma(1)}(u) X_{2 \sigma(2)}(u) \cdots X_{n \sigma(n)}(u) \\
& \qquad \operatorname{det} X(u)=u^{n p}+\bar{d}_{n 1} u^{n p-1}+\bar{d}_{n 2} u^{n p-2}+\cdots+\bar{d}_{n n p-1} u+\bar{d}_{n n p}, \quad \bar{d}_{n i} \in \bar{Y}_{p}\left(\mathfrak{g l}_{n}\right)[u]
\end{aligned}
$$

e da observação (5.4)

$$
X_{i j}(u)=\delta_{i j} u^{p}+\sum_{k=1}^{p} X_{i j}^{(k)} u^{p-k} \in \bar{Y}_{p}\left(\mathfrak{g l}_{n}\right)[u]
$$

$\operatorname{com} X_{i j}^{(0)}=\delta_{i j}$ e $X_{i j}^{(r)}=0$ para $r \geq p+1$, então

$$
\bar{d}_{n i}=\sum_{\sigma \in S_{n}} \sum_{t_{1}+t_{2}+\cdots+t_{n}=i} \operatorname{sgn}(\sigma) X_{1 \sigma(1)}^{\left(t_{1}\right)} X_{2 \sigma(2)}^{\left(t_{2}\right)} \cdots X_{n \sigma(n)}^{\left(t_{n}\right)} .
$$

Para dar alguns exemplos:

- O primeiro polinômio é:

$$
\begin{aligned}
\bar{d}_{n 1} & =\sum_{\sigma \in S_{n}} \sum_{t_{1}+t_{2}+\cdots+t_{n}=1} \operatorname{sgn}(\sigma) X_{1 \sigma(1)}^{\left(t_{1}\right)} X_{2 \sigma(2)}^{\left(t_{2}\right)} \cdots X_{n \sigma(n)}^{\left(t_{n}\right)} \\
& =X_{11}^{(1)}+X_{22}^{(1)}+\cdots+X_{n n}^{(1)} .
\end{aligned}
$$


- O segundo polinômio é:

$$
\begin{aligned}
\bar{d}_{n 2} & =\sum_{\sigma \in S_{n}} \sum_{t_{1}+t_{2}+\cdots+t_{n}=2} \operatorname{sgn}(\sigma) X_{1 \sigma(1)}^{\left(t_{1}\right)} X_{2 \sigma(2)}^{\left(t_{2}\right)} \cdots X_{n \sigma(n)}^{\left(t_{n}\right)} \\
& =\sum_{i=1}^{n} X_{i i}^{(2)}+\sum_{i=1}^{n-1} \sum_{j=i+1}^{n} X_{i i}^{(1)} X_{j j}^{(1)}-\sum_{i=1}^{n-1} \sum_{j=i+1}^{n} X_{i j}^{(1)} X_{j i}^{(1)} \\
& =\sum_{i=1}^{n} X_{i i}^{(2)}+\sum_{i=1}^{n-1} \sum_{j=i+1}^{n}\left(X_{i i}^{(1)} X_{j j}^{(1)}-X_{i j}^{(1)} X_{j i}^{(1)}\right) .
\end{aligned}
$$

- O terceiro polinômio é:

$$
\begin{aligned}
\bar{d}_{n 3}= & \sum_{\sigma \in S_{n}} \sum_{t_{1}+t_{2}+\cdots+t_{n}=3} \operatorname{sgn}(\sigma) X_{1 \sigma(1)}^{\left(t_{1}\right)} X_{2 \sigma(2)}^{\left(t_{2}\right)} \cdots X_{n \sigma(n)}^{\left(t_{n}\right)} \\
= & \sum_{i=1}^{n} X_{i i}^{(3)}+\sum_{i=1}^{n-1} \sum_{j=i+1}^{n}\left(X_{i i}^{(2)} X_{j j}^{(1)}+X_{i i}^{(1)} X_{j j}^{(2)}\right)-\sum_{i=1}^{n-1} \sum_{j=i+1}^{n}\left(X_{i j}^{(2)} X_{j i}^{(1)}+X_{i j}^{(1)} X_{j i}^{(2)}\right)+ \\
& +\sum_{\substack{A \subset\{1,2, \ldots, n\} \\
|A|=3 \\
\sigma \in S_{3}}} \operatorname{sgn}(\sigma) X_{a_{1} a_{\sigma(1)}^{(1)}} X_{a_{2} a_{\sigma(2)}}^{(1)} X_{a_{3} a_{\sigma(3)}}^{(1)} \\
= & \sum_{i=1}^{n} X_{i i}^{(3)}+\sum_{i=1}^{n-1} \sum_{j=i+1}^{n}\left(X_{i i}^{(2)} X_{j j}^{(1)}+X_{i i}^{(1)} X_{j j}^{(2)}-X_{i j}^{(2)} X_{j i}^{(1)}-X_{i j}^{(1)} X_{j i}^{(2)}\right)+ \\
& +\sum_{\substack{A \subset\{1,2, \ldots, n\} \\
|A|,=3 \\
\sigma \in S_{3}}} \operatorname{sgn}(\sigma) X_{a_{1} a_{\sigma(1)}}^{(1)} X_{a_{2} a_{\sigma(2)}}^{(1)} X_{a_{3} a_{\sigma(3)},}^{(1)},
\end{aligned}
$$

onde $A=\left\{a_{1}, a_{2}, a_{3}\right\} \operatorname{com} a_{1}<a_{2}<a_{3}$.

Observação 5.7. Vale a pena ressaltar, que temos a seguinte expressão ${ }^{2}$ para os polinômios $\bar{d}_{n i}$. Para qualquer $i \in\{1,2, \ldots, n p\}$ consideremos os diagramas de Young para $i$

$$
\lambda=\left(\lambda_{1}, \lambda_{2}, \ldots, \lambda_{r}\right)
$$

com $r \leq n$ e $\lambda_{1} \leq p$, ou seja, $\lambda_{1}, \lambda_{2}, \ldots, \lambda_{r} \in \mathbb{Z}_{>0}$ tais que

$$
\begin{gathered}
p \geq \lambda_{1} \geq \lambda_{2} \geq \ldots \geq \lambda_{r}>0, \\
\lambda_{1}+\lambda_{2}+\ldots+\lambda_{r}=i,
\end{gathered}
$$$$
1 \leq r \leq n \text {. }
$$

Denotemos o conjunto de todos os diagramas de Young $\lambda=\left(\lambda_{1}, \lambda_{2}, \ldots, \lambda_{r}\right)$ de $i$ com comprimento $r e$ $\lambda_{1} \leq p$ por

$$
\Omega_{r}(i)
$$

Assim podemos ver que

$$
\bar{d}_{n i}=\sum_{j=1}^{n} \sum_{\substack{A \subset\{1,2, \ldots, n\} \\|A|=j \\ \sigma \in S_{j}}} \sum_{\substack{\lambda \in \Omega_{j}(i) \\ \sigma^{\prime} \in S_{j}}} \operatorname{sgn}(\sigma) X_{a_{1} a_{\sigma(1)}}^{\left(\lambda_{\sigma^{\prime}(1)}\right)} X_{a_{2} a_{\sigma(2)}}^{\left(\lambda_{\sigma^{\prime}(2)}\right)} \cdots X_{a_{j} a_{\sigma(j)} a^{\prime}}^{\left(\lambda_{\sigma^{\prime}(j)}\right)}
$$

onde $A=\left\{a_{1}, a_{2}, \ldots, a_{j}\right\}$ com $a_{1}<a_{2}<\cdots<a_{j}, \lambda=\left(\lambda_{1}, \lambda_{2}, \ldots, \lambda_{r}\right)$ e $S_{j}$ as permutações de $\{1,2, \ldots, j\}$.

\footnotetext{
${ }^{2}$ Neste trabalho não iremos usar esta expressão dos polinômios $\bar{d}_{n i}$.
} 


\subsection{Variedade de Gelfand-Tsetlin para Yangians restritos $Y_{p}\left(\mathfrak{g l}_{3}\right)$}

Notemos que a variedade de Gelfand-Tsetlin $\mathfrak{G}$ para $Y_{p}\left(\mathfrak{g l}_{n}\right)$ tem polinômios da forma

$$
\begin{aligned}
\bar{d}_{11} & =X_{11}^{(1)}, \\
\bar{d}_{21} & =X_{11}^{(1)}+X_{22}^{(1)}, \\
\bar{d}_{31} & =X_{11}^{(1)}+X_{22}^{(1)}+X_{33}^{(1)}, \\
& \vdots \\
\bar{d}_{n 1} & =X_{11}^{(1)}+X_{22}^{(1)}+\cdots+X_{n n}^{(1)},
\end{aligned}
$$

mas, claramente

$$
V\left(\bar{d}_{11}, \bar{d}_{21}, \ldots, \bar{d}_{n 1}\right)=V\left(X_{11}^{(1)}, X_{22}^{(1)}, \ldots, X_{n n}^{(1)}\right) .
$$

Portanto, gostaríamos de melhorar os polinômios que determinam a variedade de Gelfand-Tsetlin. Com este objetivo, vamos entender como é a expressão dos polinômios $\bar{d}_{i j}$ e para isso estudaremos os polinômios até a subálgebra $Y_{p}\left(\mathfrak{g l}_{3}\right)$, pois como vamos ver, a combinatória apartir deste caso não é nada simples.

Lema 5.8. Os polinômios que determinam a variedade de Gelfand-Tsetlin $\mathfrak{G}$ para $Y_{p}\left(\mathfrak{g l}_{3}\right)$ são:

$$
\begin{aligned}
& \bar{d}_{1 i}=X_{11}^{(i)}, \quad i=1,2, \ldots, p \\
& \bar{d}_{21}=X_{11}^{(1)}+X_{22}^{(1)} \\
& \bar{d}_{2 i}=X_{11}^{(i)}+X_{22}^{(i)}+\sum_{t=1}^{i-1} X_{11}^{(t)} X_{22}^{(i-t)}-\sum_{t=1}^{i-1} X_{12}^{(t)} X_{21}^{(i-t)}, \quad i=2,3, \ldots, p \\
& \bar{d}_{2 p+i}=\sum_{t=i}^{p} X_{11}^{(t)} X_{22}^{(p+i-t)}-\sum_{t=i}^{p} X_{12}^{(t)} X_{21}^{(p+i-t)}, \quad i=1,2,3, \ldots, p \\
& \bar{d}_{31}=X_{11}^{(1)}+X_{22}^{(1)}+X_{33}^{(1)} \\
& \bar{d}_{32}=X_{11}^{(2)}+X_{22}^{(2)}+X_{33}^{(2)}+X_{11}^{(1)} X_{22}^{(1)}+X_{11}^{(1)} X_{33}^{(1)}+X_{22}^{(1)} X_{33}^{(1)}-X_{23}^{(1)} X_{32}^{(1)}-X_{12}^{(1)} X_{21}^{(1)}-X_{13}^{(1)} X_{31}^{(1)} \\
& \bar{d}_{3 i}=X_{33}^{(i)}+X_{22}^{(i)}+X_{11}^{(i)}+\sum_{s=1}^{i-1}\left(X_{22}^{(s)} X_{33}^{(i-s)}-X_{23}^{(s)} X_{32}^{(i-s)}\right)+ \\
& +\sum_{s=1}^{i-1}\left(X_{11}^{(s)} X_{33}^{(i-s)}-X_{13}^{(s)} X_{31}^{(i-s)}\right)+\sum_{s=1}^{i-1}\left(X_{11}^{(s)} X_{22}^{(i-s)}-X_{12}^{(s)} X_{21}^{(i-s)}\right)+ \\
& +\sum_{r=1}^{i-2} \sum_{s=1}^{i-r-1}\left\{X_{11}^{(r)} X_{22}^{(s)} X_{33}^{(i-r-s)}-X_{11}^{(r)} X_{23}^{(s)} X_{32}^{(i-r-s)}-X_{12}^{(r)} X_{21}^{(s)} X_{33}^{(i-r-s)}-\right. \\
& \left.-X_{13}^{(r)} X_{22}^{(s)} X_{31}^{(i-r-s)}+X_{12}^{(r)} X_{23}^{(s)} X_{31}^{(i-r-s)}+X_{13}^{(r)} X_{21}^{(s)} X_{32}^{(i-r-s)}\right\}, i=3,4, \ldots, p \\
& \bar{d}_{3 p+1}=\sum_{s=1}^{p}\left(X_{22}^{(s)} X_{33}^{(p+1-s)}-X_{23}^{(s)} X_{32}^{(p+1-s)}\right)+\sum_{s=1}^{p}\left(X_{11}^{(s)} X_{33}^{(p+1-s)}-X_{13}^{(s)} X_{31}^{(p+1-s)}\right)+ \\
& +\sum_{s=1}^{p}\left(X_{11}^{(s)} X_{22}^{(p+1-s)}-X_{12}^{(s)} X_{21}^{(p+1-s)}\right)+ \\
& +\sum_{r=1}^{p-1} \sum_{s=1}^{p-r}\left\{X_{11}^{(r)} X_{22}^{(s)} X_{33}^{(p+1-r-s)}-X_{11}^{(r)} X_{23}^{(s)} X_{32}^{(p+1-r-s)}-X_{12}^{(r)} X_{21}^{(s)} X_{33}^{(p+1-r-s)}-\right. \\
& \left.-X_{13}^{(r)} X_{22}^{(s)} X_{31}^{(p+1-r-s)}+X_{12}^{(r)} X_{23}^{(s)} X_{31}^{(p+1-r-s)}+X_{13}^{(r)} X_{21}^{(s)} X_{32}^{(p+1-r-s)}\right\}
\end{aligned}
$$




$$
\begin{aligned}
\bar{d}_{3 p+i}= & \sum_{s=i}^{p} X_{22}^{(s)} X_{33}^{(p+i-s)}-\sum_{s=i}^{p} X_{23}^{(s)} X_{32}^{(p+i-s)}+\sum_{s=i}^{p} X_{11}^{(s)} X_{33}^{(p+i-s)}- \\
& -\sum_{s=i}^{p} X_{13}^{(s)} X_{31}^{(p+i-s)}+\sum_{s=i}^{p} X_{11}^{(s)} X_{22}^{(p+i-s)}-\sum_{s=i}^{p} X_{12}^{(s)} X_{21}^{(p+i-s)}+ \\
& +\sum_{r=1}^{i-1} \sum_{s=i-r}^{p}\left\{X_{11}^{(r)} X_{22}^{(s)} X_{33}^{(p+i-r-s)}-X_{11}^{(r)} X_{23}^{(s)} X_{32}^{(p+i-r-s)}-X_{12}^{(r)} X_{21}^{(s)} X_{33}^{(p+i-r-s)}-\right. \\
& \left.-X_{13}^{(r)} X_{22}^{(s)} X_{31}^{(p+i-r-s)}+X_{12}^{(r)} X_{23}^{(s)} X_{31}^{(p+i-r-s)}+X_{13}^{(r)} X_{21}^{(s)} X_{32}^{(p+i-r-s)}\right\}+ \\
& +\sum_{r=i}^{p} \sum_{s=1}^{p+i-r-1}\left\{X_{11}^{(r)} X_{22}^{(s)} X_{33}^{(p+i-r-s)}-X_{11}^{(r)} X_{23}^{(s)} X_{32}^{(p+i-r-s)}-X_{12}^{(r)} X_{21}^{(s)} X_{33}^{(p+i-r-s)}-\right. \\
& \left.-X_{13}^{(r)} X_{22}^{(s)} X_{31}^{(p+i-r-s)}+X_{12}^{(r)} X_{23}^{(s)} X_{31}^{(p+i-r-s)}+X_{13}^{(r)} X_{21}^{(s)} X_{32}^{(p+i-r-s)}\right\}, i=2,3, \ldots, p \\
& \sum_{r=i}^{p} \sum_{s=p+i-r}^{p}\left\{X_{11}^{(r)} X_{22}^{(s)} X_{33}^{(2 p+i-r-s)}-X_{11}^{(r)} X_{23}^{(s)} X_{32}^{(2 p+i-r-s)}-X_{12}^{(r)} X_{21}^{(s)} X_{33}^{(2 p+i-r-s)}-\right. \\
& \left.-X_{13}^{(r)} X_{22}^{(s)} X_{31}^{(2 p+i-r-s)}+X_{12}^{(r)} X_{23}^{(s)} X_{31}^{(2 p+i-r-s)}+X_{13}^{(r)} X_{21}^{(s)} X_{32}^{(2 p+i-r-s)}\right\}, i=1,2, \ldots, p
\end{aligned}
$$

Demonstração: Claramente

$$
\bar{d}_{1 i}=X_{11}^{(i)}, \quad i=1,2, \ldots, p
$$

e

$$
\begin{aligned}
\bar{d}_{21} & =X_{11}^{(1)}+X_{22}^{(1)} \\
\bar{d}_{2 i} & =X_{11}^{(i)}+X_{22}^{(i)}+\sum_{\substack{t_{1}+t_{2}=i \\
t_{1}, t_{2} \neq 0}} X_{11}^{\left(t_{1}\right)} X_{22}^{\left(t_{2}\right)}-\sum_{\substack{t_{1}+t_{2}=i \\
t_{1}, t_{2} \neq 0}} X_{12}^{\left(t_{1}\right)} X_{21}^{\left(t_{2}\right)}, \quad i=2,3, \ldots, p \\
& =X_{11}^{(i)}+X_{22}^{(i)}+\sum_{t=1}^{i-1} X_{11}^{(t)} X_{22}^{(i-t)}-\sum_{t=1}^{i-1} X_{12}^{(t)} X_{21}^{(i-t)}, \quad i=2,3, \ldots, p .
\end{aligned}
$$

Para $i=1,2,3, \ldots, p$

$$
\begin{aligned}
\bar{d}_{2 p+i} & =\sum_{\substack{t_{1}+t_{2}=p+i \\
i \leq t_{1}, t_{2} \leq p}} X_{11}^{\left(t_{1}\right)} X_{22}^{\left(t_{2}\right)}-\sum_{\substack{t_{1}+t_{2}=p+i \\
i \leq t_{1}, t_{2} \leq p}} X_{12}^{\left(t_{1}\right)} X_{21}^{\left(t_{2}\right)} \text { (pois } X_{i j}^{(r)}=0, \forall r>p \text { ) } \\
& =\sum_{t=i}^{p} X_{11}^{(t)} X_{22}^{(p+i-t)}-\sum_{t=i}^{p} X_{12}^{(t)} X_{21}^{(p+i-t)} .
\end{aligned}
$$

Dado que, para $i=1,2, \ldots, p$

$$
\begin{aligned}
\bar{d}_{3 i}= & \sum_{\sigma \in S_{3}} \sum_{t_{1}+t_{2}+t_{3}=i} \operatorname{sgn}(\sigma) X_{1 \sigma(1)}^{\left(t_{1}\right)} X_{2 \sigma(2)}^{\left(t_{2}\right)} X_{3 \sigma(3)}^{\left(t_{3}\right)} \\
= & \sum_{t_{1}+t_{2}+t_{3}=i}\left\{X_{11}^{\left(t_{1}\right)} X_{22}^{\left(t_{2}\right)} X_{33}^{\left(t_{3}\right)}-X_{11}^{\left(t_{1}\right)} X_{23}^{\left(t_{2}\right)} X_{32}^{\left(t_{3}\right)}-X_{12}^{\left(t_{1}\right)} X_{21}^{\left(t_{2}\right)} X_{33}^{\left(t_{3}\right)}-X_{13}^{\left(t_{1}\right)} X_{22}^{\left(t_{2}\right)} X_{31}^{\left(t_{3}\right)}+\right. \\
& \left.+X_{12}^{\left(t_{1}\right)} X_{23}^{\left(t_{2}\right)} X_{31}^{\left(t_{3}\right)}+X_{13}^{\left(t_{1}\right)} X_{21}^{\left(t_{2}\right)} X_{32}^{\left(t_{3}\right)}\right\}
\end{aligned}
$$


então, pela proposição ${ }^{3}$ (A.2),

$$
\begin{aligned}
\bar{d}_{31}= & X_{11}^{(1)}+X_{22}^{(1)}+X_{33}^{(1)} \\
\bar{d}_{32}= & X_{11}^{(2)}+X_{22}^{(2)}+X_{33}^{(2)}+X_{11}^{(1)} X_{22}^{(1)}+X_{11}^{(1)} X_{33}^{(1)}+X_{22}^{(1)} X_{33}^{(1)}-X_{23}^{(1)} X_{32}^{(1)}-X_{12}^{(1)} X_{21}^{(1)}-X_{13}^{(1)} X_{31}^{(1)} \\
\bar{d}_{3 i}= & X_{33}^{(i)}+X_{22}^{(i)}+X_{11}^{(i)}+\sum_{s=1}^{i-1}\left(X_{22}^{(s)} X_{33}^{(i-s)}-X_{23}^{(s)} X_{32}^{(i-s)}\right)+ \\
& +\sum_{s=1}^{i-1}\left(X_{11}^{(s)} X_{33}^{(i-s)}-X_{13}^{(s)} X_{31}^{(i-s)}\right)+\sum_{s=1}^{i-1}\left(X_{11}^{(s)} X_{22}^{(i-s)}-X_{12}^{(s)} X_{21}^{(i-s)}\right)+ \\
& +\sum_{r=1}^{i-2} \sum_{s=1}^{i-r-1}\left\{X_{11}^{(r)} X_{22}^{(s)} X_{33}^{(i-r-s)}-X_{11}^{(r)} X_{23}^{(s)} X_{32}^{(i-r-s)}-X_{12}^{(r)} X_{21}^{(s)} X_{33}^{(i-r-s)}-\right. \\
& \left.-X_{13}^{(r)} X_{22}^{(s)} X_{31}^{(i-r-s)}+X_{12}^{(r)} X_{23}^{(s)} X_{31}^{(i-r-s)}+X_{13}^{(r)} X_{21}^{(s)} X_{32}^{(i-r-s)}\right\}, i=3,4, \ldots, p .
\end{aligned}
$$

Similarmente, dado que, para $i=1,2, \ldots, p$

$$
\begin{aligned}
\bar{d}_{3 p+i}= & \left.\sum_{\substack{\sigma \in S_{3} \\
t_{1}+t_{2}+t_{3}=p+i \\
0 \leq t_{1}, t_{2}, t_{3} \leq p}} \operatorname{sgn}(\sigma) X_{1 \sigma(1)}^{\left(t_{1}\right)} X_{2 \sigma(2)}^{\left(t_{2}\right)} X_{3 \sigma(3)}^{\left(t_{3}\right)} \text { (pois } X_{i j}^{(r)}=0, \forall r>p\right) \\
= & \sum_{\substack{t_{1}+t_{2}+t_{3}=p+i \\
0 \leq t_{1}, t_{2}, t_{3} \leq p}}\left\{X_{11}^{\left(t_{1}\right)} X_{22}^{\left(t_{2}\right)} X_{33}^{\left(t_{3}\right)}-X_{11}^{\left(t_{1}\right)} X_{23}^{\left(t_{2}\right)} X_{32}^{\left(t_{3}\right)}-X_{12}^{\left(t_{1}\right)} X_{21}^{\left(t_{2}\right)} X_{33}^{\left(t_{3}\right)}-\right. \\
& \left.-X_{13}^{\left(t_{1}\right)} X_{22}^{\left(t_{2}\right)} X_{31}^{\left(t_{3}\right)}+X_{12}^{\left(t_{1}\right)} X_{23}^{\left(t_{2}\right)} X_{31}^{\left(t_{3}\right)}+X_{13}^{\left(t_{1}\right)} X_{21}^{\left(t_{2}\right)} X_{32}^{\left(t_{3}\right)}\right\},
\end{aligned}
$$

então, da proposição (A.3), segue que

$$
\begin{aligned}
\bar{d}_{3 p+1}= & \sum_{t_{1}+t_{2}+t_{3}=p+1}\left\{X_{11}^{\left(t_{1}\right)} X_{22}^{\left(t_{2}\right)} X_{33}^{\left(t_{3}\right)}-X_{11}^{\left(t_{1}\right)} X_{23}^{\left(t_{2}\right)} X_{32}^{\left(t_{3}\right)}-X_{12}^{\left(t_{1}\right)} X_{21}^{\left(t_{2}\right)} X_{33}^{\left(t_{3}\right)}-\right. \\
& \left.-X_{13}^{\left(t_{1}\right)} X_{22}^{\left(t_{2}\right)} X_{31}^{\left(t_{3}\right)}+X_{12}^{\left(t_{1}\right)} X_{23}^{\left(t_{2}\right)} X_{31}^{\left(t_{3}\right)}+X_{13}^{\left(t_{1}\right)} X_{21}^{\left(t_{2}\right)} X_{32}^{\left(t_{3}\right)}\right\} \\
= & X_{11}^{(0)} \sum_{s=1}^{p}\left(X_{22}^{(s)} X_{33}^{(p+1-s)}-X_{23}^{(s)} X_{32}^{(p+1-s)}\right)+X_{22}^{(0)} \sum_{s=1}^{p}\left(X_{11}^{(s)} X_{33}^{(p+1-s)}-X_{13}^{(s)} X_{31}^{(p+1-s)}\right)+ \\
& +X_{33}^{(0)} \sum_{s=1}^{p}\left(X_{11}^{(s)} X_{22}^{(p+1-s)}-X_{12}^{(s)} X_{21}^{(p+1-s)}\right)+ \\
& +\sum_{r=1}^{p-1} \sum_{s=1}^{p-r}\left\{X_{11}^{(r)} X_{22}^{(s)} X_{33}^{(p+1-r-s)}-X_{11}^{(r)} X_{23}^{(s)} X_{32}^{(p+1-r-s)}-X_{12}^{(r)} X_{21}^{(s)} X_{33}^{(p+1-r-s)}-\right. \\
& \left.-X_{13}^{(r)} X_{22}^{(s)} X_{31}^{(p+1-r-s)}+X_{12}^{(r)} X_{23}^{(s)} X_{31}^{(p+1-r-s)}+X_{13}^{(r)} X_{21}^{(s)} X_{32}^{(p+1-r-s)}\right\} \\
= & \sum_{s=1}^{p}\left(X_{22}^{(s)} X_{33}^{(p+1-s)}-X_{23}^{(s)} X_{32}^{(p+1-s)}\right)+\sum_{s=1}^{p}\left(X_{11}^{(s)} X_{33}^{(p+1-s)}-X_{13}^{(s)} X_{31}^{(p+1-s)}\right)+ \\
& +\sum_{s=1}^{p}\left(X_{11}^{(s)} X_{22}^{(p+1-s)}-X_{12}^{(s)} X_{21}^{(p+1-s)}\right)+ \\
& +\sum_{r=1}^{p-1} \sum_{s=1}^{p-r}\left\{X_{11}^{(r)} X_{22}^{(s)} X_{33}^{(p+1-r-s)}-X_{11}^{(r)} X_{23}^{(s)} X_{32}^{(p+1-r-s)}-X_{12}^{(r)} X_{21}^{(s)} X_{33}^{(p+1-r-s)}-\right.
\end{aligned}
$$

${ }^{3}$ Quando aparecer uma referência na forma (A.-), significa que esta referência está no apêndice (A). 


$$
\begin{aligned}
& \left.-X_{13}^{(r)} X_{22}^{(s)} X_{31}^{(p+1-r-s)}+X_{12}^{(r)} X_{23}^{(s)} X_{31}^{(p+1-r-s)}+X_{13}^{(r)} X_{21}^{(s)} X_{32}^{(p+1-r-s)}\right\} \\
\bar{d}_{3 p+i}= & \sum_{s=i}^{p} X_{22}^{(s)} X_{33}^{(p+i-s)}-\sum_{s=i}^{p} X_{23}^{(s)} X_{32}^{(p+i-s)}+\sum_{s=i}^{p} X_{11}^{(s)} X_{33}^{(p+i-s)}- \\
& -\sum_{s=i}^{p} X_{13}^{(s)} X_{31}^{(p+i-s)}+\sum_{s=i}^{p} X_{11}^{(s)} X_{22}^{(p+i-s)}-\sum_{s=i}^{p} X_{12}^{(s)} X_{21}^{(p+i-s)}+ \\
& +\sum_{r=1}^{i-1} \sum_{s=i-r}^{p}\left\{X_{11}^{(r)} X_{22}^{(s)} X_{33}^{(p+i-r-s)}-X_{11}^{(r)} X_{23}^{(s)} X_{32}^{(p+i-r-s)}-X_{12}^{(r)} X_{21}^{(s)} X_{33}^{(p+i-r-s)}-\right. \\
& \left.-X_{13}^{(r)} X_{22}^{(s)} X_{31}^{(p+i-r-s)}+X_{12}^{(r)} X_{23}^{(s)} X_{31}^{(p+i-r-s)}+X_{13}^{(r)} X_{21}^{(s)} X_{32}^{(p+i-r-s)}\right\}+ \\
& +\sum_{r=i}^{p} \sum_{s=1}^{p+i-r-1}\left\{X_{11}^{(r)} X_{22}^{(s)} X_{33}^{(p+i-r-s)}-X_{11}^{(r)} X_{23}^{(s)} X_{32}^{(p+i-r-s)}-X_{12}^{(r)} X_{21}^{(s)} X_{33}^{(p+i-r-s)}-\right. \\
& \left.-X_{13}^{(r)} X_{22}^{(s)} X_{31}^{(p+i-r-s)}+X_{12}^{(r)} X_{23}^{(s)} X_{31}^{(p+i-r-s)}+X_{13}^{(r)} X_{21}^{(s)} X_{32}^{(p+i-r-s)}\right\}, i=2,3, \ldots, p .
\end{aligned}
$$

Finalmente, pela proposição (A.4), para cada $i=1,2, \ldots, p$

$$
\begin{aligned}
\bar{d}_{32 p+i}= & \left.\sum_{\sigma \in S_{3}} \sum_{\substack{t_{1}+t_{2}+t_{3}=2 p+i \\
0 \leq t_{1}, t_{2}, t_{3} \leq p}} \operatorname{sgn}(\sigma) X_{1 \sigma(1)}^{\left(t_{1}\right)} X_{2 \sigma(2)}^{\left(t_{2}\right)} X_{3 \sigma(3)}^{\left(t_{3}\right)} \text { (pois } X_{i j}^{(r)}=0, \forall r>p\right) \\
= & \sum_{\substack{t_{1}+t_{2}+t_{3}=2 p+i \\
0 \leq t_{1}, t_{2}, t_{3} \leq p}}\left\{X_{11}^{\left(t_{1}\right)} X_{22}^{\left(t_{2}\right)} X_{33}^{\left(t_{3}\right)}-X_{11}^{\left(t_{1}\right)} X_{23}^{\left(t_{2}\right)} X_{32}^{\left(t_{3}\right)}-X_{12}^{\left(t_{1}\right)} X_{21}^{\left(t_{2}\right)} X_{33}^{\left(t_{3}\right)}-\right. \\
& \left.-X_{13}^{\left(t_{1}\right)} X_{22}^{\left(t_{2}\right)} X_{31}^{\left(t_{3}\right)}+X_{12}^{\left(t_{1}\right)} X_{23}^{\left(t_{2}\right)} X_{31}^{\left(t_{3}\right)}+X_{13}^{\left(t_{1}\right)} X_{21}^{\left(t_{2}\right)} X_{32}^{\left(t_{3}\right)}\right\} \\
= & \sum_{r=i}^{p} \sum_{s=p+i-r}^{p}\left\{X_{11}^{(r)} X_{22}^{(s)} X_{33}^{(2 p+i-r-s)}-X_{11}^{(r)} X_{23}^{(s)} X_{32}^{(2 p+i-r-s)}-X_{12}^{(r)} X_{21}^{(s)} X_{33}^{(2 p+i-r-s)}-\right. \\
& \left.-X_{13}^{(r)} X_{22}^{(s)} X_{31}^{(2 p+i-r-s)}+X_{12}^{(r)} X_{23}^{(s)} X_{31}^{(2 p+i-r-s)}+X_{13}^{(r)} X_{21}^{(s)} X_{32}^{(2 p+i-r-s)}\right\} .
\end{aligned}
$$

Como consequência deste lema, vamos poder apresentar a variedade de Gelfand-Tsetlin até a subálgebra $Y_{p}\left(\mathfrak{g l}_{3}\right)$ de uma forma um pouco mais acessível.

\section{Proposição 5.9.}

$$
V\left(\left\{\bar{d}_{i j}\right\} \begin{array}{c}
i=1,2,3 \\
j=1,2, \ldots, i p
\end{array}\right)=V\left(\left\{p_{i j}\right\}_{\substack{i=1,2,3 \\
j=1,2, \ldots, i p}}\right) \subset k^{9 p}
$$

em que

$$
\begin{aligned}
p_{1 i} & =X_{11}^{(i)}, \quad i=1,2, \ldots, p \\
p_{21} & =X_{22}^{(1)} \\
p_{2 i} & =X_{22}^{(i)}-\sum_{t=1}^{i-1} X_{12}^{(t)} X_{21}^{(i-t)}, \quad i=2,3, \ldots, p \\
p_{2 p+i} & =\sum_{t=i}^{p} X_{12}^{(t)} X_{21}^{(p+i-t)}, \quad i=1,2, \ldots, p \\
p_{22 p} & =X_{12}^{(p)} X_{21}^{(p)} \\
p_{31} & =X_{33}^{(1)}
\end{aligned}
$$




$$
\begin{aligned}
p_{32}= & X_{33}^{(2)}-X_{23}^{(1)} X_{32}^{(1)}-X_{13}^{(1)} X_{31}^{(1)} \\
p_{33}= & X_{33}^{(3)}-X_{23}^{(1)} X_{32}^{(2)}-X_{23}^{(2)} X_{32}^{(1)}-X_{13}^{(1)} X_{31}^{(2)}-X_{13}^{(2)} X_{31}^{(1)}+X_{12}^{(1)} X_{23}^{(1)} X_{31}^{(1)}+X_{13}^{(1)} X_{21}^{(1)} X_{32}^{(1)} \\
p_{3 i}= & -\sum_{r=1}^{i-2} \sum_{s=2}^{i-r-1} X_{13}^{(r)} X_{22}^{(s)} X_{31}^{(i-r-s)}+X_{33}^{(i)}-\sum_{s=1}^{i-1}\left(X_{13}^{(s)} X_{31}^{(i-s)}+X_{23}^{(s)} X_{32}^{(i-s)}\right)+ \\
& +\sum_{r=1}^{i-2} \sum_{s=1}^{i-r-1}\left\{X_{12}^{(r)} X_{23}^{(s)} X_{31}^{(i-r-s)}+X_{13}^{(r)} X_{21}^{(s)} X_{32}^{(i-r-s)}\right\}, i=4,5, \ldots, p \\
p_{3 p+1}= & \sum_{r=1}^{p-1} \sum_{s=1}^{p-r}\left\{X_{12}^{(r)} X_{23}^{(s)} X_{31}^{(p+1-r-s)}+X_{13}^{(r)} X_{21}^{(s)} X_{32}^{(p+1-r-s)}\right\}- \\
& -\sum_{s=1}^{p}\left(X_{13}^{(s)} X_{31}^{(p+1-s)}+X_{23}^{(s)} X_{32}^{(p+1-s)}\right)-\sum_{r=1}^{p-1} \sum_{s=2}^{p-r} X_{13}^{(r)} X_{22}^{(s)} X_{31}^{(p+1-r-s)} \\
p_{3 p+i}= & -\sum_{s=i}^{p} X_{23}^{(s)} X_{32}^{(p+i-s)}-\sum_{s=i}^{p} X_{13}^{(s)} X_{31}^{(p+i-s)}+ \\
& +\sum_{r=1}^{i-1} \sum_{s=i-r}^{p}\left\{-X_{13}^{(r)} X_{22}^{(s)} X_{31}^{(p+i-r-s)}+X_{12}^{(r)} X_{23}^{(s)} X_{31}^{(p+i-r-s)}+X_{13}^{(r)} X_{21}^{(s)} X_{32}^{(p+i-r-s)}\right\}+ \\
& +\sum_{r=i}^{p} \sum_{s=1}^{p+i-r-1}\left\{-X_{13}^{(r)} X_{22}^{(s)} X_{31}^{(p+i-r-s)}+X_{12}^{(r)} X_{23}^{(s)} X_{31}^{(p+i-r-s)}+X_{13}^{(r)} X_{21}^{(s)} X_{32}^{(p+i-r-s)}\right\}, \\
& i=2,3, \ldots, p \\
p_{32 p+i}= & \sum_{r=i}^{p} \sum_{s=p+i-r}^{p}\left\{-X_{13}^{(r)} X_{22}^{(s)} X_{31}^{(2 p+i-r-s)}+X_{12}^{(r)} X_{23}^{(s)} X_{31}^{(2 p+i-r-s)}+X_{13}^{(r)} X_{21}^{(s)} X_{32}^{(2 p+i-r-s)}\right\}, i=1,2, \ldots, p .
\end{aligned}
$$

Demonstração: Claramente

$$
\begin{aligned}
& \bar{d}_{1 i}=X_{11}^{(i)}=p_{1 i}, \quad i=1,2, \ldots, p . \\
& \bar{d}_{21}=X_{11}^{(1)}+X_{22}^{(1)}=p_{11}+p_{21} .
\end{aligned}
$$

Para $i=2,3, \ldots, p$

$$
\begin{aligned}
\bar{d}_{2 i} & =X_{11}^{(i)}+X_{22}^{(i)}+\sum_{t=1}^{i-1} X_{11}^{(t)} X_{22}^{(i-t)}-\sum_{t=1}^{i-1} X_{12}^{(t)} X_{21}^{(i-t)} \\
& =X_{11}^{(i)}+\sum_{t=1}^{i-1} X_{11}^{(t)} X_{22}^{(i-t)}+X_{22}^{(i)}-\sum_{t=1}^{i-1} X_{12}^{(t)} X_{21}^{(i-t)} \\
& =p_{1 i}+\sum_{t=1}^{i-1} p_{1 t} X_{22}^{(i-t)}+p_{2 i} .
\end{aligned}
$$

Similarmente

$$
\begin{aligned}
\bar{d}_{2 p+i} & =\sum_{t=i}^{p} X_{11}^{(t)} X_{22}^{(p+i-t)}-\sum_{t=i}^{p} X_{12}^{(t)} X_{21}^{(p+i-t)}=\sum_{t=i}^{p} p_{1 t} X_{22}^{(p+i-t)}-p_{2 p+i}, \quad i=1,2, \ldots, p \\
\bar{d}_{22 p} & =X_{11}^{(p)} X_{22}^{(p)}-X_{12}^{(p)} X_{21}^{(p)}=p_{1 p} X_{22}^{(p)}-p_{22 p} .
\end{aligned}
$$

\section{Continuando com o mesmo raciocinio}

$$
\begin{aligned}
& \bar{d}_{31}=X_{11}^{(1)}+X_{22}^{(1)}+X_{33}^{(1)}=p_{11}+p_{21}+p_{31} \\
& \bar{d}_{32}=X_{11}^{(2)}+X_{22}^{(2)}+X_{33}^{(2)}+X_{11}^{(1)} X_{22}^{(1)}+X_{11}^{(1)} X_{33}^{(1)}+X_{22}^{(1)} X_{33}^{(1)}-X_{23}^{(1)} X_{32}^{(1)}-X_{12}^{(1)} X_{21}^{(1)}-X_{13}^{(1)} X_{31}^{(1)}
\end{aligned}
$$




$$
\begin{aligned}
& =X_{11}^{(2)}+X_{22}^{(2)}-X_{12}^{(1)} X_{21}^{(1)}+X_{11}^{(1)} X_{22}^{(1)}+X_{11}^{(1)} X_{33}^{(1)}+X_{22}^{(1)} X_{33}^{(1)}+X_{33}^{(2)}-X_{23}^{(1)} X_{32}^{(1)}-X_{13}^{(1)} X_{31}^{(1)} \\
& =p_{12}+p_{22}+p_{11} X_{22}^{(1)}+p_{11} X_{33}^{(1)}+p_{21} X_{33}^{(1)}+p_{32} .
\end{aligned}
$$

Para cada $i=3,4, \ldots, p$

$$
\begin{aligned}
& \bar{d}_{3 i}=X_{33}^{(i)}+X_{22}^{(i)}+X_{11}^{(i)}+\sum_{s=1}^{i-1}\left(X_{22}^{(s)} X_{33}^{(i-s)}-X_{23}^{(s)} X_{32}^{(i-s)}\right)+ \\
& +\sum_{s=1}^{i-1}\left(X_{11}^{(s)} X_{33}^{(i-s)}-X_{13}^{(s)} X_{31}^{(i-s)}\right)+\sum_{s=1}^{i-1}\left(X_{11}^{(s)} X_{22}^{(i-s)}-X_{12}^{(s)} X_{21}^{(i-s)}\right)+ \\
& +\sum_{r=1}^{i-2} \sum_{s=1}^{i-r-1}\left\{X_{11}^{(r)} X_{22}^{(s)} X_{33}^{(i-r-s)}-X_{11}^{(r)} X_{23}^{(s)} X_{32}^{(i-r-s)}-X_{12}^{(r)} X_{21}^{(s)} X_{33}^{(i-r-s)}-\right. \\
& \left.-X_{13}^{(r)} X_{22}^{(s)} X_{31}^{(i-r-s)}+X_{12}^{(r)} X_{23}^{(s)} X_{31}^{(i-r-s)}+X_{13}^{(r)} X_{21}^{(s)} X_{32}^{(i-r-s)}\right\} \\
& =X_{33}^{(i)}+X_{22}^{(i)}+X_{11}^{(i)}+\sum_{s=1}^{i-1} X_{22}^{(s)} X_{33}^{(i-s)}-\sum_{s=1}^{i-1} X_{23}^{(s)} X_{32}^{(i-s)}+ \\
& +\sum_{s=1}^{i-1}\left(X_{11}^{(s)} X_{33}^{(i-s)}+X_{11}^{(s)} X_{22}^{(i-s)}\right)-\sum_{s=1}^{i-1} X_{13}^{(s)} X_{31}^{(i-s)}-\sum_{s=1}^{i-1} X_{12}^{(s)} X_{21}^{(i-s)}+ \\
& +\sum_{r=1}^{i-2} \sum_{s=1}^{i-r-1}\left\{X_{11}^{(r)} X_{22}^{(s)} X_{33}^{(i-r-s)}-X_{11}^{(r)} X_{23}^{(s)} X_{32}^{(i-r-s)}\right\}-\sum_{r=1}^{i-2} \sum_{s=1}^{i-r-1} X_{12}^{(r)} X_{21}^{(s)} X_{33}^{(i-r-s)}+ \\
& +\sum_{r=1}^{i-2} \sum_{s=1}^{i-r-1}\left\{-X_{13}^{(r)} X_{22}^{(s)} X_{31}^{(i-r-s)}+X_{12}^{(r)} X_{23}^{(s)} X_{31}^{(i-r-s)}+X_{13}^{(r)} X_{21}^{(s)} X_{32}^{(i-r-s)}\right\} \\
& =X_{11}^{(i)}+\sum_{s=1}^{i-1}\left(X_{11}^{(s)} X_{33}^{(i-s)}+X_{11}^{(s)} X_{22}^{(i-s)}\right)+\sum_{r=1}^{i-2} \sum_{s=1}^{i-r-1}\left\{X_{11}^{(r)} X_{22}^{(s)} X_{33}^{(i-r-s)}-X_{11}^{(r)} X_{23}^{(s)} X_{32}^{(i-r-s)}\right\}+ \\
& +X_{22}^{(i)}-\sum_{s=1}^{i-1} X_{12}^{(s)} X_{21}^{(i-s)}+\sum_{s=1}^{i-1} X_{22}^{(s)} X_{33}^{(i-s)}-\sum_{r=1}^{i-2} \sum_{s=1}^{i-r-1} X_{12}^{(r)} X_{21}^{(s)} X_{33}^{(i-r-s)}+X_{33}^{(i)}-\sum_{s=1}^{i-1} X_{13}^{(s)} X_{31}^{(i-s)}- \\
& -\sum_{s=1}^{i-1} X_{23}^{(s)} X_{32}^{(i-s)}+\sum_{r=1}^{i-2} \sum_{s=1}^{i-r-1}\left\{-X_{13}^{(r)} X_{22}^{(s)} X_{31}^{(i-r-s)}+X_{12}^{(r)} X_{23}^{(s)} X_{31}^{(i-r-s)}+X_{13}^{(r)} X_{21}^{(s)} X_{32}^{(i-r-s)}\right\} \\
& =p_{1 i}+\sum_{s=1}^{i-1} p_{1 s}\left(X_{33}^{(i-s)}+X_{22}^{(i-s)}\right)+\sum_{r=1}^{i-2} \sum_{s=1}^{i-r-1} p_{1 r}\left(X_{22}^{(s)} X_{33}^{(i-r-s)}-X_{23}^{(s)} X_{32}^{(i-r-s)}\right)+ \\
& +p_{2 i}+\sum_{s=1}^{i-1} X_{22}^{(s)} X_{33}^{(i-s)}-\sum_{r=1}^{i-2} \sum_{s=1}^{i-r-1} X_{12}^{(r)} X_{21}^{(s)} X_{33}^{(i-r-s)}+p_{3 i} \\
& =p_{1 i}+\sum_{s=1}^{i-1} p_{1 s}\left(X_{33}^{(i-s)}+X_{22}^{(i-s)}\right)+\sum_{r=1}^{i-2} \sum_{s=1}^{i-r-1} p_{1 r}\left(X_{22}^{(s)} X_{33}^{(i-r-s)}-X_{23}^{(s)} X_{32}^{(i-r-s)}\right)+ \\
& +p_{2 i}+\sum_{s=1}^{i-1} X_{22}^{(s)} X_{33}^{(i-s)}-\sum_{\substack{r+s+t=i \\
r, s, t \neq 0}} X_{12}^{(r)} X_{21}^{(s)} X_{33}^{(t)}+p_{3 i} \text { (proposição (A.1)) } \\
& =p_{1 i}+\sum_{s=1}^{i-1} p_{1 s}\left(X_{33}^{(i-s)}+X_{22}^{(i-s)}\right)+\sum_{r=1}^{i-2} \sum_{s=1}^{i-r-1} p_{1 r}\left(X_{22}^{(s)} X_{33}^{(i-r-s)}-X_{23}^{(s)} X_{32}^{(i-r-s)}\right)+ \\
& +p_{2 i}+\sum_{r=1}^{i-1} X_{22}^{(i-r)} X_{33}^{(r)}-\sum_{\substack{r+s+t=i \\
r, s, t \neq 0}} X_{33}^{(r)} X_{12}^{(s)} X_{21}^{(t)}+p_{3 i} \text { (por comutatividade) }
\end{aligned}
$$




$$
\begin{aligned}
= & p_{1 i}+\sum_{s=1}^{i-1} p_{1 s}\left(X_{33}^{(i-s)}+X_{22}^{(i-s)}\right)+\sum_{r=1}^{i-2} \sum_{s=1}^{i-r-1} p_{1 r}\left(X_{22}^{(s)} X_{33}^{(i-r-s)}-X_{23}^{(s)} X_{32}^{(i-r-s)}\right)+ \\
& +p_{2 i}+X_{22}^{(1)} X_{33}^{(i-1)}+\sum_{r=1}^{i-2} X_{22}^{(i-r)} X_{33}^{(r)}-\sum_{r=1}^{i-2} \sum_{s=1}^{i-r-1} X_{33}^{(r)} X_{12}^{(s)} X_{21}^{(i-r-s)}+p_{3 i} \text { (proposição (A.1)) } \\
= & p_{1 i}+\sum_{s=1}^{i-1} p_{1 s}\left(X_{33}^{(i-s)}+X_{22}^{(i-s)}\right)+\sum_{r=1}^{i-2} \sum_{s=1}^{i-r-1} p_{1 r}\left(X_{22}^{(s)} X_{33}^{(i-r-s)}-X_{23}^{(s)} X_{32}^{(i-r-s)}\right)+ \\
& +p_{2 i}+p_{21} X_{33}^{(i-1)}+\sum_{r=1}^{i-2}\left(X_{22}^{(i-r)}-\sum_{s=1}^{i-r-1} X_{12}^{(s)} X_{21}^{(i-r-s)}\right) X_{33}^{(r)}+p_{3 i} \\
= & p_{1 i}+\sum_{s=1}^{i-1} p_{1 s}\left(X_{33}^{(i-s)}+X_{22}^{(i-s)}\right)+\sum_{r=1}^{i-2} \sum_{s=1}^{i-r-1} p_{1 r}\left(X_{22}^{(s)} X_{33}^{(i-r-s)}-X_{23}^{(s)} X_{32}^{(i-r-s)}\right)+ \\
& +p_{2 i}+p_{21} X_{33}^{(i-1)}+\sum_{r=1}^{i-2} p_{2 i-r} X_{33}^{(r)}+p_{3 i} .
\end{aligned}
$$

\section{Análogamente}

$$
\begin{aligned}
\bar{d}_{3 p+1}= & \sum_{s=1}^{p}\left(X_{22}^{(s)} X_{33}^{(p+1-s)}-X_{23}^{(s)} X_{32}^{(p+1-s)}\right)+\sum_{s=1}^{p}\left(X_{11}^{(s)} X_{33}^{(p+1-s)}-X_{13}^{(s)} X_{31}^{(p+1-s)}\right)+ \\
& +\sum_{s=1}^{p}\left(X_{11}^{(s)} X_{22}^{(p+1-s)}-X_{12}^{(s)} X_{21}^{(p+1-s)}\right)+ \\
& +\sum_{r=1}^{p-1} \sum_{s=1}^{p-r}\left\{X_{11}^{(r)} X_{22}^{(s)} X_{33}^{(p+1-r-s)}-X_{11}^{(r)} X_{23}^{(s)} X_{32}^{(p+1-r-s)}-X_{12}^{(r)} X_{21}^{(s)} X_{33}^{(p+1-r-s)}-\right. \\
& \left.-X_{13}^{(r)} X_{22}^{(s)} X_{31}^{(p+1-r-s)}+X_{12}^{(r)} X_{23}^{(s)} X_{31}^{(p+1-r-s)}+X_{13}^{(r)} X_{21}^{(s)} X_{32}^{(p+1-r-s)}\right\} \\
= & \sum_{s=1}^{p} X_{22}^{(s)} X_{33}^{(p+1-s)}+\sum_{s=1}^{p} X_{11}^{(s)} X_{33}^{(p+1-s)}-\sum_{s=1}^{p}\left(X_{23}^{(s)} X_{32}^{(p+1-s)}+X_{13}^{(s)} X_{31}^{(p+1-s)}\right)+ \\
& +\sum_{s=1}^{p} X_{11}^{(s)} X_{22}^{(p+1-s)}-\sum_{s=1}^{p} X_{12}^{(s)} X_{21}^{(p+1-s)}+ \\
& +\sum_{r=1}^{p-1} \sum_{s=1}^{p-r}\left(X_{11}^{(r)} X_{22}^{(s)} X_{33}^{(p+1-r-s)}-X_{11}^{(r)} X_{23}^{(s)} X_{32}^{(p+1-r-s)}\right)-\sum_{r=1}^{p-1} \sum_{s=1}^{p-r} X_{12}^{(r)} X_{21}^{(s)} X_{33}^{(p+1-r-s)}+ \\
& +\sum_{r=1}^{p-1} \sum_{s=1}^{p-r}\left(-X_{13}^{(r)} X_{22}^{(s)} X_{31}^{(p+1-r-s)}+X_{12}^{(r)} X_{23}^{(s)} X_{31}^{(p+1-r-s)}+X_{13}^{(r)} X_{21}^{(s)} X_{32}^{(p+1-r-s)}\right) \\
= & \sum_{s=1}^{p} X_{11}^{(s)} X_{33}^{(p+1-s)}+\sum_{s=1}^{p} X_{11}^{(s)} X_{22}^{(p+1-s)}+\sum_{r=1}^{p-1} \sum_{s=1}^{p-r}\left(X_{11}^{(r)} X_{22}^{(s)} X_{33}^{(p+1-r-s)}-X_{11}^{(r)} X_{23}^{(s)} X_{32}^{(p+1-r-s)}\right)+ \\
& +\sum_{s=1}^{p} X_{22}^{(s)} X_{33}^{(p+1-s)}-\sum_{r=1}^{p-1} \sum_{s=1}^{p-r} X_{12}^{(r)} X_{21}^{(s)} X_{33}^{(p+1-r-s)}-\sum_{s=1}^{p} X_{12}^{(s)} X_{21}^{(p+1-s)}+ \\
& -\sum_{s=1}^{p}\left(X_{23}^{(s)} X_{32}^{(p+1-s)}+X_{13}^{(s)} X_{31}^{(p+1-s)}\right)+ \\
& +\sum_{3=1}^{p-1} \sum_{s=1}^{p-r}\left(-X_{13}^{(r)} X_{22}^{(s)} X_{31}^{(p+1-r-s)}+X_{12}^{(r)} X_{23}^{(s)} X_{31}^{(p+1-r-s)}+X_{13}^{(r)} X_{21}^{(s)} X_{32}^{(p+1-r-s)}\right) \\
& \left.X_{22}^{(p+1-s)}\right)+\sum_{r=1}^{p-1} \sum_{s=1}^{p-r} p_{1 r}\left(X_{22}^{(s)} X_{33}^{(p+1-r-s)}-X_{23}^{(s)} X_{32}^{(p+1-r-s)}\right)+ \\
& \\
& \\
&
\end{aligned}
$$




$$
\begin{aligned}
& +\sum_{s=1}^{p} X_{22}^{(s)} X_{33}^{(p+1-s)}-\sum_{r=1}^{p-1} \sum_{s=1}^{p-r} X_{12}^{(r)} X_{21}^{(s)} X_{33}^{(p+1-r-s)}-p_{2 p+1}+p_{3 p+1} \\
& =\sum_{s=1}^{p} p_{1 s}\left(X_{33}^{(p+1-s)}+X_{22}^{(p+1-s)}\right)+\sum_{r=1}^{p-1} \sum_{s=1}^{p-r} p_{1 r}\left(X_{22}^{(s)} X_{33}^{(p+1-r-s)}-X_{23}^{(s)} X_{32}^{(p+1-r-s)}\right)+ \\
& +\sum_{s=1}^{p} X_{22}^{(s)} X_{33}^{(p+1-s)}-\sum_{\substack{r+s+t=p+1 \\
1 \leq r, s, t \leq p}} X_{12}^{(r)} X_{21}^{(s)} X_{33}^{(t)}-p_{2 p+1}+p_{3 p+1} \quad \text { (proposição (A.1)) } \\
& =\sum_{s=1}^{p} p_{1 s}\left(X_{33}^{(p+1-s)}+X_{22}^{(p+1-s)}\right)+\sum_{r=1}^{p-1} \sum_{s=1}^{p-r} p_{1 r}\left(X_{22}^{(s)} X_{33}^{(p+1-r-s)}-X_{23}^{(s)} X_{32}^{(p+1-r-s)}\right)+ \\
& +\sum_{r=1}^{p} X_{22}^{(p+1-r)} X_{33}^{(r)}-\sum_{\substack{r+s+t=p+1 \\
1 \leq r, s, t \leq p}} X_{33}^{(r)} X_{12}^{(s)} X_{21}^{(t)}-p_{2 p+1}+p_{3 p+1} \quad \text { (por comutatividade) } \\
& =\sum_{s=1}^{p} p_{1 s}\left(X_{33}^{(p+1-s)}+X_{22}^{(p+1-s)}\right)+\sum_{r=1}^{p-1} \sum_{s=1}^{p-r} p_{1 r}\left(X_{22}^{(s)} X_{33}^{(p+1-r-s)}-X_{23}^{(s)} X_{32}^{(p+1-r-s)}\right)+ \\
& +X_{22}^{(1)} X_{33}^{(p)}+\sum_{r=1}^{p-1} X_{22}^{(p+1-r)} X_{33}^{(r)}-\sum_{r=1}^{p-1} \sum_{s=1}^{p-r} X_{33}^{(r)} X_{12}^{(s)} X_{21}^{(p+1-r-s)}-p_{2 p+1}+p_{3 p+1} \quad \text { (proposição (A.1)) } \\
& =\sum_{s=1}^{p} p_{1 s}\left(X_{33}^{(p+1-s)}+X_{22}^{(p+1-s)}\right)+\sum_{r=1}^{p-1} \sum_{s=1}^{p-r} p_{1 r}\left(X_{22}^{(s)} X_{33}^{(p+1-r-s)}-X_{23}^{(s)} X_{32}^{(p+1-r-s)}\right)+ \\
& +p_{21} X_{33}^{(p)}+\sum_{r=1}^{p-1} X_{33}^{(r)}\left(X_{22}^{(p+1-r)}-\sum_{s=1}^{p-r} X_{12}^{(s)} X_{21}^{(p+1-r-s)}\right)-p_{2 p+1}+p_{3 p+1} \\
& =\sum_{s=1}^{p} p_{1 s}\left(X_{33}^{(p+1-s)}+X_{22}^{(p+1-s)}\right)+\sum_{r=1}^{p-1} \sum_{s=1}^{p-r} p_{1 r}\left(X_{22}^{(s)} X_{33}^{(p+1-r-s)}-X_{23}^{(s)} X_{32}^{(p+1-r-s)}\right)+ \\
& +p_{21} X_{33}^{(p)}+\sum_{r=1}^{p-1} X_{33}^{(r)} p_{2 p+1-r}-p_{2 p+1}+p_{3 p+1} \text {. }
\end{aligned}
$$

Para $i=2,3, \ldots, p$

$$
\begin{aligned}
\bar{d}_{3 p+i}= & \sum_{s=i}^{p} X_{22}^{(s)} X_{33}^{(p+i-s)}-\sum_{s=i}^{p} X_{23}^{(s)} X_{32}^{(p+i-s)}+\sum_{s=i}^{p} X_{11}^{(s)} X_{33}^{(p+i-s)}- \\
& -\sum_{s=i}^{p} X_{13}^{(s)} X_{31}^{(p+i-s)}+\sum_{s=i}^{p} X_{11}^{(s)} X_{22}^{(p+i-s)}-\sum_{s=i}^{p} X_{12}^{(s)} X_{21}^{(p+i-s)}+ \\
& +\sum_{r=1}^{i-1} \sum_{s=i-r}^{p}\left\{X_{11}^{(r)} X_{22}^{(s)} X_{33}^{(p+i-r-s)}-X_{11}^{(r)} X_{23}^{(s)} X_{32}^{(p+i-r-s)}-X_{12}^{(r)} X_{21}^{(s)} X_{33}^{(p+i-r-s)}-\right. \\
& \left.-X_{13}^{(r)} X_{22}^{(s)} X_{31}^{(p+i-r-s)}+X_{12}^{(r)} X_{23}^{(s)} X_{31}^{(p+i-r-s)}+X_{13}^{(r)} X_{21}^{(s)} X_{32}^{(p+i-r-s)}\right\}+ \\
& +\sum_{r=i}^{p} \sum_{s=1}^{p+i-r-1}\left\{X_{11}^{(r)} X_{22}^{(s)} X_{33}^{(p+i-r-s)}-X_{11}^{(r)} X_{23}^{(s)} X_{32}^{(p+i-r-s)}-X_{12}^{(r)} X_{21}^{(s)} X_{33}^{(p+i-r-s)}-\right. \\
& \left.-X_{13}^{(r)} X_{22}^{(s)} X_{31}^{(p+i-r-s)}+X_{12}^{(r)} X_{23}^{(s)} X_{31}^{(p+i-r-s)}+X_{13}^{(r)} X_{21}^{(s)} X_{32}^{(p+i-r-s)}\right\} \\
= & \sum_{s=i}^{p} X_{22}^{(s)} X_{33}^{(p+i-s)}-\sum_{s=i}^{p} X_{23}^{(s)} X_{32}^{(p+i-s)}+\sum_{s=i}^{p} X_{11}^{(s)} X_{33}^{(p+i-s)}-
\end{aligned}
$$




$$
\begin{aligned}
& -\sum_{s=i}^{p} X_{13}^{(s)} X_{31}^{(p+i-s)}+\sum_{s=i}^{p} X_{11}^{(s)} X_{22}^{(p+i-s)}-\sum_{s=i}^{p} X_{12}^{(s)} X_{21}^{(p+i-s)}+ \\
& +\sum_{r=1}^{i-1} \sum_{s=i-r}^{p}\left(X_{11}^{(r)} X_{22}^{(s)} X_{33}^{(p+i-r-s)}-X_{11}^{(r)} X_{23}^{(s)} X_{32}^{(p+i-r-s)}\right)-\sum_{r=1}^{i-1} \sum_{s=i-r}^{p} X_{12}^{(r)} X_{21}^{(s)} X_{33}^{(p+i-r-s)}+ \\
& \sum_{r=1}^{i-1} \sum_{s=i-r}^{p}\left(-X_{13}^{(r)} X_{22}^{(s)} X_{31}^{(p+i-r-s)}+X_{12}^{(r)} X_{23}^{(s)} X_{31}^{(p+i-r-s)}+X_{13}^{(r)} X_{21}^{(s)} X_{32}^{(p+i-r-s)}\right)+ \\
& +\sum_{r=i}^{p} \sum_{s=1}^{p+i-r-1}\left(X_{11}^{(r)} X_{22}^{(s)} X_{33}^{(p+i-r-s)}-X_{11}^{(r)} X_{23}^{(s)} X_{32}^{(p+i-r-s)}\right)-\sum_{r=i}^{p} \sum_{s=1}^{p+i-r-1} X_{12}^{(r)} X_{21}^{(s)} X_{33}^{(p+i-r-s)}+ \\
& +\sum_{r=i}^{p} \sum_{s=1}^{p+i-r-1}\left(-X_{13}^{(r)} X_{22}^{(s)} X_{31}^{(p+i-r-s)}+X_{12}^{(r)} X_{23}^{(s)} X_{31}^{(p+i-r-s)}+X_{13}^{(r)} X_{21}^{(s)} X_{32}^{(p+i-r-s)}\right) \\
& =\sum_{s=i}^{p} X_{11}^{(s)} X_{22}^{(p+i-s)}+\sum_{s=i}^{p} X_{11}^{(s)} X_{33}^{(p+i-s)}+\sum_{r=1}^{i-1} \sum_{s=i-r}^{p}\left(X_{11}^{(r)} X_{22}^{(s)} X_{33}^{(p+i-r-s)}-X_{11}^{(r)} X_{23}^{(s)} X_{32}^{(p+i-r-s)}\right)+ \\
& +\sum_{r=i}^{p} \sum_{s=1}^{p+i-r-1}\left(X_{11}^{(r)} X_{22}^{(s)} X_{33}^{(p+i-r-s)}-X_{11}^{(r)} X_{23}^{(s)} X_{32}^{(p+i-r-s)}\right)-\sum_{s=i}^{p} X_{12}^{(s)} X_{21}^{(p+i-s)}+ \\
& +\sum_{s=i}^{p} X_{22}^{(s)} X_{33}^{(p+i-s)}-\sum_{r=1}^{i-1} \sum_{s=i-r}^{p} X_{12}^{(r)} X_{21}^{(s)} X_{33}^{(p+i-r-s)}-\sum_{r=i}^{p} \sum_{s=1}^{p+i-r-1} X_{12}^{(r)} X_{21}^{(s)} X_{33}^{(p+i-r-s)}+ \\
& -\sum_{s=i}^{p} X_{13}^{(s)} X_{31}^{(p+i-s)}-\sum_{s=i}^{p} X_{23}^{(s)} X_{32}^{(p+i-s)}+ \\
& +\sum_{r=1}^{i-1} \sum_{s=i-r}^{p}\left(-X_{13}^{(r)} X_{22}^{(s)} X_{31}^{(p+i-r-s)}+X_{12}^{(r)} X_{23}^{(s)} X_{31}^{(p+i-r-s)}+X_{13}^{(r)} X_{21}^{(s)} X_{32}^{(p+i-r-s)}\right)+ \\
& +\sum_{r=i}^{p} \sum_{s=1}^{p+i-r-1}\left(-X_{13}^{(r)} X_{22}^{(s)} X_{31}^{(p+i-r-s)}+X_{12}^{(r)} X_{23}^{(s)} X_{31}^{(p+i-r-s)}+X_{13}^{(r)} X_{21}^{(s)} X_{32}^{(p+i-r-s)}\right) \\
& =\sum_{s=i}^{p} p_{1 s} X_{22}^{(p+i-s)}+\sum_{s=i}^{p} p_{1 s} X_{33}^{(p+i-s)}+\sum_{r=1}^{i-1} \sum_{s=i-r}^{p}\left(p_{1 r} X_{22}^{(s)} X_{33}^{(p+i-r-s)}-p_{1 r} X_{23}^{(s)} X_{32}^{(p+i-r-s)}\right)+ \\
& +\sum_{r=i}^{p} \sum_{s=1}^{p+i-r-1}\left(p_{1 r} X_{22}^{(s)} X_{33}^{(p+i-r-s)}-p_{1 r} X_{23}^{(s)} X_{32}^{(p+i-r-s)}\right)-p_{2 i}+ \\
& +\sum_{s=i}^{p} X_{22}^{(s)} X_{33}^{(p+i-s)}-\sum_{r=1}^{i-1} \sum_{s=i-r}^{p} X_{12}^{(r)} X_{21}^{(s)} X_{33}^{(p+i-r-s)}-\sum_{r=i}^{p} \sum_{s=1}^{p+i-r-1} X_{12}^{(r)} X_{21}^{(s)} X_{33}^{(p+i-r-s)}+p_{3 p+i} \\
& =\sum_{s=i}^{p} p_{1 s}\left(X_{22}^{(p+i-s)}+X_{33}^{(p+i-s)}\right)+\sum_{r=1}^{i-1} \sum_{s=i-r}^{p} p_{1 r}\left(X_{22}^{(s)} X_{33}^{(p+i-r-s)}-X_{23}^{(s)} X_{32}^{(p+i-r-s)}\right)+ \\
& +\sum_{r=i}^{p} \sum_{s=1}^{p+i-r-1} p_{1 r}\left(X_{22}^{(s)} X_{33}^{(p+i-r-s)}-X_{23}^{(s)} X_{32}^{(p+i-r-s)}\right)-p_{2 i}+ \\
& +\sum_{s=i}^{p} X_{22}^{(s)} X_{33}^{(p+i-s)}-\sum_{r=1}^{i-1} \sum_{s=i-r}^{p} X_{12}^{(r)} X_{21}^{(s)} X_{33}^{(p+i-r-s)}-\sum_{r=i}^{p} \sum_{s=1}^{p+i-r-1} X_{12}^{(r)} X_{21}^{(s)} X_{33}^{(p+i-r-s)}+p_{3 p+i} \\
& =\sum_{s=i}^{p} p_{1 s}\left(X_{22}^{(p+i-s)}+X_{33}^{(p+i-s)}\right)+\sum_{r=1}^{i-1} \sum_{s=i-r}^{p} p_{1 r}\left(X_{22}^{(s)} X_{33}^{(p+i-r-s)}-X_{23}^{(s)} X_{32}^{(p+i-r-s)}\right)+ \\
& +\sum_{r=i}^{p} \sum_{s=1}^{p+i-r-1} p_{1 r}\left(X_{22}^{(s)} X_{33}^{(p+i-r-s)}-X_{23}^{(s)} X_{32}^{(p+i-r-s)}\right)-p_{2 i}+
\end{aligned}
$$




$$
\begin{aligned}
& +\sum_{s=i}^{p} X_{22}^{(s)} X_{33}^{(p+i-s)}-\sum_{\substack{r+s+t=p+i \\
1 \leq r, s, t \leq p}} X_{12}^{(r)} X_{21}^{(s)} X_{33}^{(t)}+p_{3 p+i} \quad \text { (proposição (A.1)) } \\
& =\sum_{s=i}^{p} p_{1 s}\left(X_{22}^{(p+i-s)}+X_{33}^{(p+i-s)}\right)+\sum_{r=1}^{i-1} \sum_{s=i-r}^{p} p_{1 r}\left(X_{22}^{(s)} X_{33}^{(p+i-r-s)}-X_{23}^{(s)} X_{32}^{(p+i-r-s)}\right)+ \\
& +\sum_{r=i}^{p} \sum_{s=1}^{p+i-r-1} p_{1 r}\left(X_{22}^{(s)} X_{33}^{(p+i-r-s)}-X_{23}^{(s)} X_{32}^{(p+i-r-s)}\right)-p_{2 i}+ \\
& +\sum_{s=i}^{p} X_{22}^{(s)} X_{33}^{(p+i-s)}-\sum_{\substack{r+s+t=p+i \\
1 \leq r, s, t \leq p}} X_{33}^{(r)} X_{12}^{(s)} X_{21}^{(t)}+p_{3 p+i} \quad \text { (por comutatividade) } \\
& =\sum_{s=i}^{p} p_{1 s}\left(X_{22}^{(p+i-s)}+X_{33}^{(p+i-s)}\right)+\sum_{r=1}^{i-1} \sum_{s=i-r}^{p} p_{1 r}\left(X_{22}^{(s)} X_{33}^{(p+i-r-s)}-X_{23}^{(s)} X_{32}^{(p+i-r-s)}\right)+ \\
& +\sum_{r=i}^{p} \sum_{s=1}^{p+i-r-1} p_{1 r}\left(X_{22}^{(s)} X_{33}^{(p+i-r-s)}-X_{23}^{(s)} X_{32}^{(p+i-r-s)}\right)-p_{2 i}+\sum_{r=i}^{p} X_{22}^{(p+i-r)} X_{33}^{(r)}- \\
& -\sum_{r=1}^{i-1} \sum_{s=i-r}^{p} X_{33}^{(r)} X_{12}^{(s)} X_{21}^{(p+i-r-s)}-\sum_{r=i}^{p} \sum_{s=1}^{p+i-r-1} X_{33}^{(r)} X_{12}^{(s)} X_{21}^{(p+i-r-s)}+p_{3 p+i} \quad \text { (proposição (A.1)) } \\
& =\sum_{s=i}^{p} p_{1 s}\left(X_{22}^{(p+i-s)}+X_{33}^{(p+i-s)}\right)+\sum_{r=1}^{i-1} \sum_{s=i-r}^{p} p_{1 r}\left(X_{22}^{(s)} X_{33}^{(p+i-r-s)}-X_{23}^{(s)} X_{32}^{(p+i-r-s)}\right)+ \\
& +\sum_{r=i}^{p} \sum_{s=1}^{p+i-r-1} p_{1 r}\left(X_{22}^{(s)} X_{33}^{(p+i-r-s)}-X_{23}^{(s)} X_{32}^{(p+i-r-s)}\right)-p_{2 i}+\sum_{r=i}^{p} X_{22}^{(p+i-r)} X_{33}^{(r)}- \\
& -\sum_{r=i}^{p} \sum_{s=1}^{p+i-r-1} X_{33}^{(r)} X_{12}^{(s)} X_{21}^{(p+i-r-s)}-\sum_{r=1}^{i-1} \sum_{s=i-r}^{p} X_{33}^{(r)} X_{12}^{(s)} X_{21}^{(p+i-r-s)}+p_{3 p+i} \\
& =\sum_{s=i}^{p} p_{1 s}\left(X_{22}^{(p+i-s)}+X_{33}^{(p+i-s)}\right)+\sum_{r=1}^{i-1} \sum_{s=i-r}^{p} p_{1 r}\left(X_{22}^{(s)} X_{33}^{(p+i-r-s)}-X_{23}^{(s)} X_{32}^{(p+i-r-s)}\right)+ \\
& +\sum_{r=i}^{p} \sum_{s=1}^{p+i-r-1} p_{1 r}\left(X_{22}^{(s)} X_{33}^{(p+i-r-s)}-X_{23}^{(s)} X_{32}^{(p+i-r-s)}\right)-p_{2 i}+ \\
& +\sum_{r=i}^{p} X_{33}^{(r)}\left(X_{22}^{(p+i-r)}-\sum_{s=1}^{p+i-r-1} X_{12}^{(s)} X_{21}^{(p+i-r-s)}\right)-\sum_{r=1}^{i-1} \sum_{s=i-r}^{p} X_{33}^{(r)} X_{12}^{(s)} X_{21}^{(p+i-r-s)}+p_{3 p+i} \\
& =\sum_{s=i}^{p} p_{1 s}\left(X_{22}^{(p+i-s)}+X_{33}^{(p+i-s)}\right)+\sum_{r=1}^{i-1} \sum_{s=i-r}^{p} p_{1 r}\left(X_{22}^{(s)} X_{33}^{(p+i-r-s)}-X_{23}^{(s)} X_{32}^{(p+i-r-s)}\right)+ \\
& +\sum_{r=i}^{p} \sum_{s=1}^{p+i-r-1} p_{1 r}\left(X_{22}^{(s)} X_{33}^{(p+i-r-s)}-X_{23}^{(s)} X_{32}^{(p+i-r-s)}\right)-p_{2 i}+ \\
& +\sum_{r=i}^{p} X_{33}^{(r)} p_{2 p+i-r}-\sum_{r=1}^{i-1} X_{33}^{(r)} p_{2 p+i-r}+p_{3 p+i}
\end{aligned}
$$

Finalmente, para cada $i=1,2, \ldots, p$

$$
\begin{aligned}
\bar{d}_{32 p+i}= & \sum_{r=i}^{p} \sum_{s=p+i-r}^{p}\left\{X_{11}^{(r)} X_{22}^{(s)} X_{33}^{(2 p+i-r-s)}-X_{11}^{(r)} X_{23}^{(s)} X_{32}^{(2 p+i-r-s)}-X_{12}^{(r)} X_{21}^{(s)} X_{33}^{(2 p+i-r-s)}-\right. \\
& \left.-X_{13}^{(r)} X_{22}^{(s)} X_{31}^{(2 p+i-r-s)}+X_{12}^{(r)} X_{23}^{(s)} X_{31}^{(2 p+i-r-s)}+X_{13}^{(r)} X_{21}^{(s)} X_{32}^{(2 p+i-r-s)}\right\}
\end{aligned}
$$




$$
\begin{aligned}
= & \sum_{r=i}^{p} \sum_{s=p+i-r}^{p}\left(X_{11}^{(r)} X_{22}^{(s)} X_{33}^{(2 p+i-r-s)}-X_{11}^{(r)} X_{23}^{(s)} X_{32}^{(2 p+i-r-s)}\right)-\sum_{r=i}^{p} \sum_{s=p+i-r}^{p} X_{12}^{(r)} X_{21}^{(s)} X_{33}^{(2 p+i-r-s)}+ \\
& +\sum_{r=i}^{p} \sum_{s=p+i-r}^{p}\left(-X_{13}^{(r)} X_{22}^{(s)} X_{31}^{(2 p+i-r-s)}+X_{12}^{(r)} X_{23}^{(s)} X_{31}^{(2 p+i-r-s)}+X_{13}^{(r)} X_{21}^{(s)} X_{32}^{(2 p+i-r-s)}\right) \\
= & \sum_{r=i}^{p} \sum_{s=p+i-r}^{p}\left(p_{1 r} X_{22}^{(s)} X_{33}^{(2 p+i-r-s)}-p_{1 r} X_{23}^{(s)} X_{32}^{(2 p+i-r-s)}\right)-\sum_{r=i}^{p} \sum_{s=p+i-r}^{p} X_{12}^{(r)} X_{21}^{(s)} X_{33}^{(2 p+i-r-s)}+p_{32 p+i} \\
= & \sum_{r=i}^{p} \sum_{s=p+i-r}^{p} p_{1 r}\left(X_{22}^{(s)} X_{33}^{(2 p+i-r-s)}-X_{23}^{(s)} X_{32}^{(2 p+i-r-s)}\right)-\sum_{r=i}^{p} \sum_{\substack{s=p+i-r \\
r}}^{p} X_{12}^{(r)} X_{21}^{(s)} X_{33}^{(2 p+i-r-s)}+p_{32 p+i} \\
= & \sum_{r=i}^{p} \sum_{s=p+i-r}^{p} p_{1 r}\left(X_{22}^{(s)} X_{33}^{(2 p+i-r-s)}-X_{23}^{(s)} X_{32}^{(2 p+i-r-s)}\right)-\sum_{\substack{r+s+t=2 p+i \\
1 \leq r, s, t \leq p}} X_{12}^{(r)} X_{21}^{(s)} X_{33}^{(t)}+p_{32 p+i}
\end{aligned}
$$

(proposição (A.1))

$=\sum_{r=i}^{p} \sum_{s=p+i-r}^{p} p_{1 r}\left(X_{22}^{(s)} X_{33}^{(2 p+i-r-s)}-X_{23}^{(s)} X_{32}^{(2 p+i-r-s)}\right)-\sum_{\substack{r+s+t=2 p+i \\ 1 \leq r, s, t \leq p}} X_{33}^{(r)} X_{12}^{(s)} X_{21}^{(t)}+p_{32 p+i}$

(por comutatividade)

$=\sum_{r=i}^{p} \sum_{s=p+i-r}^{p} p_{1 r}\left(X_{22}^{(s)} X_{33}^{(2 p+i-r-s)}-X_{23}^{(s)} X_{32}^{(2 p+i-r-s)}\right)-\sum_{r=i}^{p} \sum_{s=p+i-r}^{p} X_{33}^{(r)} X_{12}^{(s)} X_{21}^{(2 p+i-r-s)}+p_{32 p+i}$ (proposição (A.1))

$=\sum_{r=i}^{p} \sum_{s=p+i-r}^{p} p_{1 r}\left(X_{22}^{(s)} X_{33}^{(2 p+i-r-s)}-X_{23}^{(s)} X_{32}^{(2 p+i-r-s)}\right)-\sum_{r=i}^{p} X_{33}^{(r)} p_{2 p+i-r}+p_{32 p+i}$.

Corolário 5.10. A variedade de Gelfand-Tsetlin $\mathfrak{G}$ para $Y_{p}\left(\mathfrak{g l}_{n}\right)$ pode ser descrita na forma:

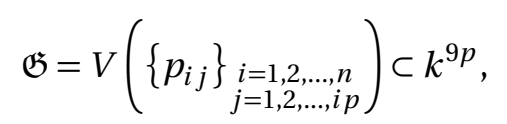

em que

$$
p_{i j}=\bar{d}_{i j}, \text { para } 4 \leq i \leq n \text { e } 1 \leq j \leq i p .
$$

Demonstração: Segue da definição (5.5) e da proposição (5.9).

\subsubsection{Equidimensionalidade para $Y_{1}\left(\mathfrak{g l}_{3}\right)$ e $Y_{2}\left(\mathfrak{g l}_{3}\right)$}

Como já dito, do corolário (4.13) segue que a variedade de Gelfand-Tsetlin $\mathfrak{G}$ para $Y_{1}\left(\mathfrak{g l}_{n}\right)$ coincide com a variedade de Gelfand-Tsetlin para $\mathfrak{g l}_{n}$. Assim, pelo Teorema de Ovsienko (3.15) temos que esta variedade é equidimensional de dimensão

$$
\operatorname{dim} \mathfrak{G}=\frac{n(n-1)}{2} .
$$

O Teorema de Ovsienko (3.15) afirma que todas as componentes irredutíveis têm a mesma dimensão, mas não diz nada sobre como é sua decomposição em componentes irredutíveis. Com o intuito de apresentar a dificuldade desse problema, temos o seguinte resultado: 
Proposição 5.11. A decomposição em componentes irredutíveis da variedade de Gelfand-Tsetlin $\mathfrak{G}$ para $Y_{1}\left(\mathfrak{g l}_{3}\right) \dot{e}$

$$
\begin{aligned}
\mathfrak{G}= & V\left(X_{11}^{(1)}, X_{22}^{(1)}, X_{12}^{(1)}, X_{33}^{(1)}, X_{23}^{(1)}, X_{13}^{(1)}\right) \cup V\left(X_{11}^{(1)}, X_{22}^{(1)}, X_{12}^{(1)}, X_{33}^{(1)}, X_{32}^{(1)}, X_{13}^{(1)}\right) \cup \\
& \cup V\left(X_{11}^{(1)}, X_{22}^{(1)}, X_{12}^{(1)}, X_{33}^{(1)}, X_{13}^{(1)} X_{31}^{(1)}+X_{23}^{(1)} X_{32}^{(1)}, X_{21}^{(1)}\right) \cup \\
& \cup V\left(X_{11}^{(1)}, X_{22}^{(1)}, X_{12}^{(1)}, X_{33}^{(1)}, X_{31}^{(1)}, X_{32}^{(1)}\right) \cup V\left(X_{11}^{(1)}, X_{22}^{(1)}, X_{21}^{(1)}, X_{33}^{(1)}, X_{13}^{(1)}, X_{23}^{(1)}\right) \cup \\
& \cup V\left(X_{11}^{(1)}, X_{22}^{(1)}, X_{21}^{(1)}, X_{33}^{(1)}, X_{31}^{(1)}, X_{23}^{(1)}\right) \cup V\left(X_{11}^{(1)}, X_{22}^{(1)}, X_{21}^{(1)}, X_{33}^{(1)}, X_{32}^{(1)}, X_{31}^{(1)}\right) .
\end{aligned}
$$

Portanto, $\mathfrak{G}$ para $Y_{1}\left(\mathfrak{g l}_{3}\right)$ é equidimensional com

$$
\operatorname{dim} \mathfrak{G}=3 .
$$

Demonstração: Segue da proposição (5.9) ou do corolário (5.10) que

$$
\mathfrak{G}=V\left(p_{11}, p_{21}, p_{22}, p_{31}, p_{32}, p_{33}\right) \subset k^{9},
$$

em que

$$
\begin{aligned}
& p_{11}=X_{11}^{(1)}, \\
& p_{21}=X_{22}^{(1)}, \\
& p_{22}=X_{12}^{(1)} X_{21}^{(1)}, \\
& p_{31}=X_{33}^{(1)}, \\
& p_{32}=X_{13}^{(1)} X_{31}^{(1)}+X_{23}^{(1)} X_{32}^{(1)}, \\
& p_{33}=-X_{13}^{(1)} \underbrace{X_{22}^{(1)}}_{=p_{21}} X_{31}^{(1)}+X_{12}^{(1)} X_{23}^{(1)} X_{31}^{(1)}+X_{13}^{(1)} X_{21}^{(1)} X_{32}^{(1)},
\end{aligned}
$$

logo

$$
\begin{aligned}
& \mathfrak{G}=V\left(X_{11}^{(1)}, X_{22}^{(1)}, X_{12}^{(1)} X_{21}^{(1)}, X_{33}^{(1)}, X_{13}^{(1)} X_{31}^{(1)}+X_{23}^{(1)} X_{32}^{(1)}, X_{12}^{(1)} X_{23}^{(1)} X_{31}^{(1)}+X_{13}^{(1)} X_{21}^{(1)} X_{32}^{(1)}\right) \\
& =V\left(X_{11}^{(1)}, X_{22}^{(1)}, X_{12}^{(1)}, X_{33}^{(1)}, X_{13}^{(1)} X_{31}^{(1)}+X_{23}^{(1)} X_{32}^{(1)}, X_{13}^{(1)} X_{21}^{(1)} X_{32}^{(1)}\right) \cup \\
& \cup V\left(X_{11}^{(1)}, X_{22}^{(1)}, X_{21}^{(1)}, X_{33}^{(1)}, X_{13}^{(1)} X_{31}^{(1)}+X_{23}^{(1)} X_{32}^{(1)}, X_{12}^{(1)} X_{23}^{(1)} X_{31}^{(1)}\right) \\
& =V\left(X_{11}^{(1)}, X_{22}^{(1)}, X_{12}^{(1)}, X_{33}^{(1)}, X_{23}^{(1)} X_{32}^{(1)}, X_{13}^{(1)}\right) \cup V\left(X_{11}^{(1)}, X_{22}^{(1)}, X_{12}^{(1)}, X_{33}^{(1)}, X_{13}^{(1)} X_{31}^{(1)}+X_{23}^{(1)} X_{32}^{(1)}, X_{21}^{(1)}\right) \cup \\
& \cup V\left(X_{11}^{(1)}, X_{22}^{(1)}, X_{12}^{(1)}, X_{33}^{(1)}, X_{13}^{(1)} X_{31}^{(1)}, X_{32}^{(1)}\right) \cup V\left(X_{11}^{(1)}, X_{22}^{(1)}, X_{21}^{(1)}, X_{33}^{(1)}, X_{13}^{(1)} X_{31}^{(1)}+X_{23}^{(1)} X_{32}^{(1)}, X_{12}^{(1)}\right) \cup \\
& \cup V\left(X_{11}^{(1)}, X_{22}^{(1)}, X_{21}^{(1)}, X_{33}^{(1)}, X_{13}^{(1)} X_{31}^{(1)}, X_{23}^{(1)}\right) \cup V\left(X_{11}^{(1)}, X_{22}^{(1)}, X_{21}^{(1)}, X_{33}^{(1)}, X_{23}^{(1)} X_{32}^{(1)}, X_{31}^{(1)}\right) \\
& =V\left(X_{11}^{(1)}, X_{22}^{(1)}, X_{12}^{(1)}, X_{33}^{(1)}, X_{23}^{(1)} X_{32}^{(1)}, X_{13}^{(1)}\right) \cup V\left(X_{11}^{(1)}, X_{22}^{(1)}, X_{12}^{(1)}, X_{33}^{(1)}, X_{13}^{(1)} X_{31}^{(1)}+X_{23}^{(1)} X_{32}^{(1)}, X_{21}^{(1)}\right) \cup \\
& \cup V\left(X_{11}^{(1)}, X_{22}^{(1)}, X_{12}^{(1)}, X_{33}^{(1)}, X_{13}^{(1)} X_{31}^{(1)}, X_{32}^{(1)}\right) \cup V\left(X_{11}^{(1)}, X_{22}^{(1)}, X_{21}^{(1)}, X_{33}^{(1)}, X_{13}^{(1)} X_{31}^{(1)}, X_{23}^{(1)}\right) \cup \\
& \cup V\left(X_{11}^{(1)}, X_{22}^{(1)}, X_{21}^{(1)}, X_{33}^{(1)}, X_{23}^{(1)} X_{32}^{(1)}, X_{31}^{(1)}\right) \\
& =V\left(X_{11}^{(1)}, X_{22}^{(1)}, X_{12}^{(1)}, X_{33}^{(1)}, X_{23}^{(1)}, X_{13}^{(1)}\right) \cup V\left(X_{11}^{(1)}, X_{22}^{(1)}, X_{12}^{(1)}, X_{33}^{(1)}, X_{32}^{(1)}, X_{13}^{(1)}\right) \cup \\
& \cup V\left(X_{11}^{(1)}, X_{22}^{(1)}, X_{12}^{(1)}, X_{33}^{(1)}, X_{13}^{(1)} X_{31}^{(1)}+X_{23}^{(1)} X_{32}^{(1)}, X_{21}^{(1)}\right) \cup \\
& \cup V\left(X_{11}^{(1)}, X_{22}^{(1)}, X_{12}^{(1)}, X_{33}^{(1)}, X_{13}^{(1)}, X_{32}^{(1)}\right) \cup V\left(X_{11}^{(1)}, X_{22}^{(1)}, X_{12}^{(1)}, X_{33}^{(1)}, X_{31}^{(1)}, X_{32}^{(1)}\right) \cup \\
& \cup V\left(X_{11}^{(1)}, X_{22}^{(1)}, X_{21}^{(1)}, X_{33}^{(1)}, X_{13}^{(1)}, X_{23}^{(1)}\right) \cup V\left(X_{11}^{(1)}, X_{22}^{(1)}, X_{21}^{(1)}, X_{33}^{(1)}, X_{31}^{(1)}, X_{23}^{(1)}\right) \cup \\
& \cup V\left(X_{11}^{(1)}, X_{22}^{(1)}, X_{21}^{(1)}, X_{33}^{(1)}, X_{23}^{(1)}, X_{31}^{(1)}\right) \cup V\left(X_{11}^{(1)}, X_{22}^{(1)}, X_{21}^{(1)}, X_{33}^{(1)}, X_{32}^{(1)}, X_{31}^{(1)}\right)
\end{aligned}
$$




$$
\begin{aligned}
= & V\left(X_{11}^{(1)}, X_{22}^{(1)}, X_{12}^{(1)}, X_{33}^{(1)}, X_{23}^{(1)}, X_{13}^{(1)}\right) \cup V\left(X_{11}^{(1)}, X_{22}^{(1)}, X_{12}^{(1)}, X_{33}^{(1)}, X_{32}^{(1)}, X_{13}^{(1)}\right) \cup \\
& \cup V\left(X_{11}^{(1)}, X_{22}^{(1)}, X_{12}^{(1)}, X_{33}^{(1)}, X_{13}^{(1)} X_{31}^{(1)}+X_{23}^{(1)} X_{32}^{(1)}, X_{21}^{(1)}\right) \cup \\
& \cup V\left(X_{11}^{(1)}, X_{22}^{(1)}, X_{12}^{(1)}, X_{33}^{(1)}, X_{31}^{(1)}, X_{32}^{(1)}\right) \cup V\left(X_{11}^{(1)}, X_{22}^{(1)}, X_{21}^{(1)}, X_{33}^{(1)}, X_{13}^{(1)}, X_{23}^{(1)}\right) \cup \\
& \cup V\left(X_{11}^{(1)}, X_{22}^{(1)}, X_{21}^{(1)}, X_{33}^{(1)}, X_{31}^{(1)}, X_{23}^{(1)}\right) \cup V\left(X_{11}^{(1)}, X_{22}^{(1)}, X_{21}^{(1)}, X_{33}^{(1)}, X_{32}^{(1)}, X_{31}^{(1)}\right) .
\end{aligned}
$$

Portanto, $\mathfrak{G}$ para $Y_{1}\left(\mathfrak{g l}_{3}\right)$ tem 7 componentes irredutíveis e cada componente tem dimensão 3.

Com o mesmo intuito, de apresentar a dificuldade da equidimensionalidade de $\mathfrak{G}$, a seguinte proposição garante a equidimensionalidade da variedade de Gelfand-Tsetlin $\mathfrak{G}$ para $Y_{2}\left(\mathfrak{g l}_{3}\right)$, apresentando sua decomposição em componentes irredutíveis. Todos os detalhes da decomposição estão no apêndice (B).

Proposição 5.12. A variedade de Gelfand-Tsetlin $\mathfrak{G}$ para $Y_{2}\left(\mathfrak{g l}_{3}\right)$ é equidimensional com

$$
\operatorname{dim} \mathfrak{G}=6 .
$$

Demonstração. A proposição (5.9) ou corolário (5.10), garantem que

$$
\mathfrak{G}=V\left(\left\{p_{i j}\right\} \underset{\substack{i=1,2,3 \\ j=1,2, \ldots, 2 i}}{ }\right) \subset k^{18}
$$

em que

$$
\begin{aligned}
p_{11}= & X_{11}^{(1)} \\
p_{12}= & X_{11}^{(2)} \\
p_{21}= & X_{22}^{(1)} \\
p_{22}= & X_{22}^{(2)}-X_{12}^{(1)} X_{21}^{(1)} \\
p_{23}= & X_{12}^{(1)} X_{21}^{(2)}+X_{12}^{(2)} X_{21}^{(1)} \\
p_{24}= & X_{12}^{(2)} X_{21}^{(2)} \\
p_{31}= & X_{33}^{(1)} \\
p_{32}= & X_{33}^{(2)}-X_{23}^{(1)} X_{32}^{(1)}-X_{13}^{(1)} X_{31}^{(1)} \\
p_{33}= & X_{12}^{(1)} X_{23}^{(1)} X_{31}^{(1)}+X_{13}^{(1)} X_{21}^{(1)} X_{32}^{(1)}-X_{13}^{(1)} X_{31}^{(2)}-X_{23}^{(1)} X_{32}^{(2)}-X_{13}^{(2)} X_{31}^{(1)}-X_{23}^{(2)} X_{32}^{(1)}-X_{13}^{(1)} \underbrace{(1)}_{22} X_{31}^{(1)} \\
p_{34}= & -X_{23}^{(2)} X_{32}^{(2)}-X_{13}^{(2)} X_{31}^{(2)}-X_{13}^{(1)} \underbrace{(1)}_{=p_{21}} X_{31}^{(2)}+X_{12}^{(1)} X_{23}^{(1)} X_{31}^{(2)}+X_{13}^{(1)} X_{21}^{(1)} X_{32}^{(2)}-X_{13}^{(1)} X_{22}^{(2)} X_{31}^{(1)}+ \\
& +X_{12}^{(1)} X_{23}^{(2)} X_{31}^{(1)}+X_{13}^{(1)} X_{21}^{(2)} X_{32}^{(1)}-X_{13}^{(2)} \underbrace{X_{22}^{(1)}}_{=p_{21}} X_{31}^{(1)}+X_{12}^{(2)} X_{23}^{(1)} X_{31}^{(1)}+X_{13}^{(2)} X_{21}^{(1)} X_{32}^{(1)} \\
p_{35}= & -X_{13}^{(1)} X_{22}^{(2)} X_{31}^{(2)}+X_{12}^{(1)} X_{23}^{(2)} X_{31}^{(2)}+X_{13}^{(1)} X_{21}^{(2)} X_{32}^{(2)}-X_{13}^{(2)} \underbrace{(1)}_{=p_{21}} X_{31}^{(2)}+X_{12}^{(2)} X_{23}^{(1)} X_{31}^{(2)}+ \\
& +X_{13}^{(2)} X_{21}^{(1)} X_{32}^{(2)}-X_{13}^{(2)} X_{22}^{(2)} X_{31}^{(1)}+X_{12}^{(2)} X_{23}^{(2)} X_{31}^{(1)}+X_{13}^{(2)} X_{21}^{(2)} X_{32}^{(1)} \\
p_{36}= & -X_{13}^{(2)} X_{22}^{(2)} X_{31}^{(2)}+X_{12}^{(2)} X_{23}^{(2)} X_{31}^{(2)}+X_{13}^{(2)} X_{21}^{(2)} X_{32}^{(2)} .
\end{aligned}
$$

Pelo apêndice (B) temos que a decomposição em componentes irredutíveis é 


$$
\mathfrak{G}=\bigcup_{i=1}^{22} C_{i}
$$

em que

$$
\begin{aligned}
& C_{1}=V\left(X_{11}^{(1)}, X_{11}^{(2)}, X_{22}^{(1)}, X_{22}^{(2)}, X_{12}^{(1)}, X_{12}^{(2)}, X_{33}^{(1)}, X_{33}^{(2)}, X_{23}^{(1)}, X_{23}^{(2)}, X_{13}^{(1)}, X_{13}^{(2)}\right), \\
& C_{2}=V\left(X_{11}^{(1)}, X_{11}^{(2)}, X_{22}^{(1)}, X_{22}^{(2)}, X_{12}^{(1)}, X_{12}^{(2)}, X_{33}^{(1)}, X_{33}^{(2)}-X_{23}^{(1)} X_{32}^{(1)}, X_{32}^{(2)}, X_{23}^{(2)}, X_{13}^{(1)}, X_{13}^{(2)}\right) \text {, } \\
& C_{3}=V\left(X_{11}^{(1)}, X_{11}^{(2)}, X_{22}^{(1)}, X_{22}^{(2)}, X_{12}^{(1)}, X_{12}^{(2)}, X_{33}^{(1)}, X_{33}^{(2)}, X_{32}^{(1)}, X_{32}^{(2)}, X_{13}^{(1)}, X_{13}^{(2)}\right) \text {, } \\
& C_{4}=V\left(X_{11}^{(1)}, X_{11}^{(2)}, X_{22}^{(1)}, X_{22}^{(2)}, X_{12}^{(1)}, X_{12}^{(2)}, X_{33}^{(1)}, X_{33}^{(2)}-X_{23}^{(1)} X_{32}^{(1)}-X_{13}^{(1)} X_{31}^{(1)}\right. \text {, } \\
& \left.-X_{23}^{(2)} X_{32}^{(1)}+X_{13}^{(1)} X_{21}^{(1)} X_{32}^{(1)}-X_{13}^{(1)} X_{31}^{(2)}, X_{32}^{(2)}, X_{21}^{(2)}, X_{13}^{(2)}\right), \\
& C_{5}=V\left(X_{11}^{(1)}, X_{11}^{(2)}, X_{22}^{(1)}, X_{22}^{(2)}, X_{12}^{(1)}, X_{12}^{(2)}, X_{33}^{(1)}, X_{33}^{(2)}-X_{23}^{(1)} X_{32}^{(1)}-X_{13}^{(1)} X_{31}^{(1)}\right. \text {, } \\
& \left.-X_{23}^{(1)} X_{32}^{(2)}-X_{13}^{(1)} X_{31}^{(2)},-X_{23}^{(2)}+X_{13}^{(1)} X_{21}^{(1)}, X_{21}^{(2)}, X_{13}^{(2)}\right), \\
& C_{6}=V\left(X_{11}^{(1)}, X_{11}^{(2)}, X_{22}^{(1)}, X_{22}^{(2)}, X_{12}^{(1)}, X_{12}^{(2)}, X_{33}^{(1)}, X_{33}^{(2)}-X_{13}^{(1)} X_{31}^{(1)}, X_{31}^{(2)}, X_{32}^{(1)}, X_{32}^{(2)}, X_{13}^{(2)}\right) \text {, } \\
& C_{7}=V\left(X_{11}^{(1)}, X_{11}^{(2)}, X_{22}^{(1)}, X_{22}^{(2)}, X_{12}^{(1)}, X_{12}^{(2)}, X_{33}^{(1)}, X_{33}^{(2)}-X_{23}^{(1)} X_{32}^{(1)}-X_{13}^{(1)} X_{31}^{(1)}\right. \text {, } \\
& \left.X_{23}^{(1)} X_{32}^{(2)}+X_{23}^{(2)} X_{32}^{(1)}+X_{13}^{(1)} X_{31}^{(2)}+X_{13}^{(2)} X_{31}^{(1)}, X_{23}^{(2)} X_{32}^{(2)}+X_{13}^{(2)} X_{31}^{(2)}, X_{21}^{(1)}, X_{21}^{(2)}\right) \\
& C_{8}=V\left(X_{11}^{(1)}, X_{11}^{(2)}, X_{22}^{(1)}, X_{22}^{(2)}, X_{12}^{(1)}, X_{12}^{(2)}, X_{33}^{(1)}, X_{33}^{(2)}-X_{23}^{(1)} X_{32}^{(1)}-X_{13}^{(1)} X_{31}^{(1)}\right. \text {, } \\
& \left.X_{23}^{(2)} X_{32}^{(1)}+X_{13}^{(2)} X_{31}^{(1)}, X_{21}^{(1)} X_{32}^{(1)}-X_{31}^{(2)}, X_{32}^{(2)}, X_{21}^{(2)}\right) \text {, } \\
& C_{9}=V\left(X_{11}^{(1)}, X_{11}^{(2)}, X_{22}^{(1)}, X_{22}^{(2)}, X_{12}^{(1)}, X_{12}^{(2)}, X_{33}^{(1)}, X_{33}^{(2)}, X_{31}^{(1)}, X_{31}^{(2)}, X_{32}^{(1)}, X_{32}^{(2)}\right) \text {, } \\
& C_{10}=V\left(X_{11}^{(1)}, X_{11}^{(2)}, X_{22}^{(1)}, X_{22}^{(2)}-X_{12}^{(1)} X_{21}^{(1)}, X_{21}^{(2)}, X_{12}^{(2)}, X_{33}^{(1)}, X_{33}^{(2)}-X_{13}^{(1)} X_{31}^{(1)}, X_{23}^{(1)}, X_{23}^{(2)}-X_{13}^{(1)} X_{21}^{(1)}, X_{31}^{(2)}, X_{13}^{(2)}\right), \\
& C_{11}=V\left(X_{11}^{(1)}, X_{11}^{(2)}, X_{22}^{(1)}, X_{22}^{(2)}-X_{12}^{(1)} X_{21}^{(1)}, X_{21}^{(2)}, X_{12}^{(2)}, X_{33}^{(1)}, X_{33}^{(2)}-X_{23}^{(1)} X_{32}^{(1)}-X_{13}^{(1)} X_{31}^{(1)}\right. \text {, } \\
& \left.X_{32}^{(2)}-X_{12}^{(1)} X_{31}^{(1)}, X_{23}^{(2)}-X_{13}^{(1)} X_{21}^{(1)}, X_{31}^{(2)}, X_{13}^{(2)}\right) \text {, } \\
& C_{12}=V\left(X_{11}^{(1)}, X_{11}^{(2)}, X_{22}^{(1)}, X_{22}^{(2)}-X_{12}^{(1)} X_{21}^{(1)}, X_{21}^{(2)}, X_{12}^{(2)}, X_{33}^{(1)}, X_{33}^{(2)}-X_{13}^{(1)} X_{31}^{(1)}, X_{32}^{(1)}, X_{32}^{(2)}-X_{12}^{(1)} X_{31}^{(1)}, X_{31}^{(2)}, X_{13}^{(2)}\right), \\
& C_{13}=V\left(X_{11}^{(1)}, X_{11}^{(2)}, X_{22}^{(1)}, X_{22}^{(2)}-X_{12}^{(1)} X_{21}^{(1)}, X_{21}^{(2)}, X_{12}^{(2)}, X_{33}^{(1)}, X_{33}^{(2)}, X_{13}^{(1)}, X_{23}^{(1)}, X_{23}^{(2)}, X_{13}^{(2)}\right) \text {, } \\
& C_{14}=V\left(X_{11}^{(1)}, X_{11}^{(2)}, X_{22}^{(1)}, X_{21}^{(1)}, X_{21}^{(2)}, X_{12}^{(2)}, X_{33}^{(1)}, X_{33}^{(2)}-X_{23}^{(1)} X_{32}^{(1)}-X_{13}^{(1)} X_{31}^{(1)}\right. \text {, } \\
& \left.X_{23}^{(1)} X_{32}^{(2)}-X_{12}^{(1)} X_{23}^{(1)} X_{31}^{(1)}+X_{13}^{(2)} X_{31}^{(1)}, X_{31}^{(2)}, X_{23}^{(2)}, X_{22}^{(2)}\right) \text {, } \\
& C_{15}=V\left(X_{11}^{(1)}, X_{11}^{(2)}, X_{22}^{(1)}, X_{21}^{(1)}, X_{21}^{(2)}, X_{12}^{(2)}, X_{33}^{(1)}, X_{33}^{(2)}-X_{23}^{(1)} X_{32}^{(1)}-X_{13}^{(1)} X_{31}^{(1)}\right. \text {, } \\
& \left.X_{23}^{(1)} X_{32}^{(2)}+X_{13}^{(1)} X_{31}^{(2)}, X_{12}^{(1)} X_{23}^{(1)}-X_{13}^{(2)}, X_{23}^{(2)}, X_{22}^{(2)}\right) \text {, } \\
& C_{16}=V\left(X_{11}^{(1)}, X_{11}^{(2)}, X_{22}^{(1)}, X_{21}^{(1)}, X_{21}^{(2)}, X_{12}^{(2)}, X_{33}^{(1)}, X_{33}^{(2)}-X_{23}^{(1)} X_{32}^{(1)}-X_{13}^{(1)} X_{31}^{(1)}\right. \text {, } \\
& \left.X_{23}^{(2)} X_{32}^{(1)}+X_{13}^{(2)} X_{31}^{(1)}, X_{32}^{(2)}-X_{12}^{(1)} X_{31}^{(1)}, X_{31}^{(2)}, X_{22}^{(2)}\right) \text {, }
\end{aligned}
$$




$$
\begin{aligned}
& C_{17}=V\left(X_{11}^{(1)}, X_{11}^{(2)}, X_{22}^{(1)}, X_{22}^{(2)}-X_{12}^{(1)} X_{21}^{(1)}, X_{21}^{(2)}, X_{12}^{(2)}, X_{33}^{(1)}, X_{33}^{(2)}, X_{31}^{(1)}, X_{32}^{(1)}, X_{32}^{(2)}, X_{31}^{(2)}\right), \\
& C_{18}=V\left(X_{11}^{(1)}, X_{11}^{(2)}, X_{22}^{(1)}, X_{22}^{(2)}, X_{21}^{(1)}, X_{21}^{(2)}, X_{33}^{(1)}, X_{33}^{(2)}, X_{32}^{(1)}, X_{32}^{(2)}, X_{31}^{(1)}, X_{31}^{(2)}\right), \\
& C_{19}=V\left(X_{11}^{(1)}, X_{11}^{(2)}, X_{22}^{(1)}, X_{22}^{(2)}, X_{21}^{(1)}, X_{21}^{(2)}, X_{33}^{(1)}, X_{33}^{(2)}-X_{32}^{(1)} X_{23}^{(1)}, X_{23}^{(2)}, X_{32}^{(2)}, X_{31}^{(1)}, X_{31}^{(2)}\right), \\
& C_{20}=V\left(X_{11}^{(1)}, X_{11}^{(2)}, X_{22}^{(1)}, X_{22}^{(2)}, X_{21}^{(1)}, X_{21}^{(2)}, X_{33}^{(1)}, X_{33}^{(2)}, X_{23}^{(1)}, X_{23}^{(2)}, X_{31}^{(1)}, X_{31}^{(2)}\right), \\
& C_{21}=V\left(X_{11}^{(1)}, X_{11}^{(2)}, X_{22}^{(1)}, X_{22}^{(2)}, X_{21}^{(1)}, X_{21}^{(2)}, X_{33}^{(1)}, X_{33}^{(2)}-X_{31}^{(1)} X_{13}^{(1)}, X_{13}^{(2)}, X_{23}^{(1)}, X_{23}^{(2)}, X_{31}^{(2)}\right), \\
& C_{22}=V\left(X_{11}^{(1)}, X_{11}^{(2)}, X_{22}^{(1)}, X_{22}^{(2)}, X_{21}^{(1)}, X_{21}^{(2)}, X_{33}^{(1)}, X_{33}^{(2)}, X_{13}^{(1)}, X_{13}^{(2)}, X_{23}^{(1)}, X_{23}^{(2)}\right) .
\end{aligned}
$$

Cada componente irredutível de $\mathfrak{G}$ tem dimensão 6.

\subsection{Uma primeira decomposição de $\mathfrak{G}$ para Yangians restritos $Y_{p}\left(\mathfrak{g l}_{n}\right)$}

Nesta seção apresentaremos uma decomposição da variedade de Gelfand-Tsetlin $\mathfrak{G}$ para Yangians $Y_{p}\left(\mathfrak{g l}_{n}\right)$. Mas, vale a pena ressaltar que como veremos nesta seção, tal decomposição não é em componentes irredutíveis.

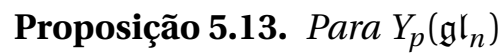

$$
\mathfrak{G}=\bigcup_{s=1}^{p+1} \mathfrak{G}_{s}
$$

em que

$$
\begin{gathered}
\mathfrak{G}_{1}:=V\left(\left\{p_{i j}\right\}_{\substack{i=1,2 \\
j=1,2, \ldots, p}} \cup\left\{X_{12}^{(i)}\right\}_{i=1}^{p} \cup\left\{p_{i j}\right\}_{\substack{i=3,4, \ldots, n \\
j=1,2, \ldots, i p}}\right) \subset k^{n^{2} p}, \\
\mathfrak{G}_{s}:=V\left(\left\{p_{i j}\right\}_{\substack{i=1,2 \\
j=1,2, \ldots, p}} \cup\left\{X_{12}^{(i)}\right\}_{i=s}^{p} \cup\left\{X_{21}^{(i)}\right\}_{i=p-(s-2)}^{p} \cup\left\{p_{i j}\right\}_{\substack{i=3,4, \ldots, n \\
j=1,2, \ldots, i p}}^{p}\right) \subset k^{n^{2} p}, \text { para } 2 \leq s \leq p, \\
\mathfrak{G}_{p+1}:=V\left(\left\{p_{i j}\right\}_{\substack{i=1,2 \\
j=1,2, \ldots, p}} \cup\left\{X_{21}^{(i)}\right\}_{i=1}^{p} \cup\left\{p_{i j}\right\}_{\substack{i=3,4, \ldots, n \\
j=1,2, \ldots, i p}}\right) \subset k^{n^{2} p} .
\end{gathered}
$$

Demonstração. Claramente $\mathfrak{G}_{s} \subseteq \mathfrak{G}$ para cada $s=1,2, \ldots, p+1$, portanto

$$
\mathfrak{G} \supseteq \bigcup_{s=1}^{p+1} \mathfrak{G}_{s}
$$

Agora, para

$$
\mathfrak{G} \subseteq \bigcup_{s=1}^{p+1} \mathfrak{G}_{s},
$$

consideremos $A=\left(a_{i j}^{(t)}\right)_{\substack{i, j=1,2,3 \\ t=1,2, \ldots, p}} \in \mathfrak{G}$ e o polinômio

$$
p_{22 p}=X_{12}^{(p)} X_{21}^{(p)}
$$

pela proposição (5.9) ou corolário (5.10),

$$
p_{22 p}(A)=a_{12}^{(p)} a_{21}^{(p)}=0,
$$

logo

$$
a_{12}^{(p)}=0 \text { ou } a_{21}^{(p)}=0 .
$$


Portanto, se $p=1$ então

$$
\mathfrak{G}=\mathfrak{G}_{1} \cup \mathfrak{G}_{2} .
$$

Novamente, se $p>1$ consideremos também o polinômio

$$
p_{22 p-1}=\sum_{t=p-1}^{p} X_{12}^{(t)} X_{21}^{(2 p-1-t)}=X_{12}^{(p-1)} X_{21}^{(p)}+X_{12}^{(p)} X_{21}^{(p-1)},
$$

como consequência da proposição (5.9) ou do corolário (5.10), temos

$$
p_{22 p-1}(A)=a_{12}^{(p-1)} a_{21}^{(p)}+a_{12}^{(p)} a_{21}^{(p-1)}=0,
$$

assim

$$
a_{12}^{(p)}=a_{12}^{(p-1)}=0 \text { ou } a_{12}^{(p)}=a_{21}^{(p)}=0 \text { ou } a_{21}^{(p)}=a_{21}^{(p-1)}=0 .
$$

Portanto, se $p=2$ então

$$
\mathfrak{G}=\mathfrak{G}_{1} \cup \mathfrak{G}_{2} \cup \mathfrak{G}_{3} .
$$

Agora, para o caso que $p>2$. Dado que $A \in \mathfrak{G}_{1}$ quando $a_{12}^{(t)}=0$ para todo $t=1,2, \ldots, p$ e $A \in \mathfrak{G}_{p+1}$ quando $a_{21}^{(t)}=0$ para todo $t=1,2, \ldots, p$, então podemos assumir que, existe $s \in\{2,3, \ldots, p\}$ tal que

$$
a_{12}^{(t)}=0, \quad \forall t \geq s \quad \text { e } \quad a_{12}^{(s-1)} \neq 0 .
$$

Como consequência do polinômio

$$
p_{p p+s-1}=\sum_{t=s-1}^{p} X_{12}^{(t)} X_{21}^{(p+s-1-t)}=X_{12}^{(s-1)} X_{21}^{(p)}+\sum_{t=s}^{p} X_{12}^{(t)} X_{21}^{(p+s-1-t)}
$$

e da proposição (5.9) ou corolário (5.10), tem-se

$$
p_{p p+s-1}(A)=a_{12}^{(s-1)} a_{21}^{(p)}+\sum_{t=s}^{p} a_{12}^{(t)} a_{21}^{(p+s-1-t)}=0
$$

e assim

$$
a_{21}^{(p)}=0
$$

implica que, se $s=2$ então $A \in \mathfrak{G}_{2}$.

Continuando com o mesmo raciocinio para $3 \leq s \leq p$, se

$$
a_{21}^{(t)}=0, \forall t \geq j \text { para algum } j \in\{p-s+3, p-s+4, \ldots, p\} .
$$

Dado que ${ }^{4} 1 \leq s-p+j-2 \leq p$, então temos o polinômio

$$
\begin{aligned}
p_{p s+j-2} & =p_{p p+s-p+j-2}=\sum_{t=s-p+j-2}^{p} X_{12}^{(t)} X_{21}^{(p+s-p+j-2-t)}=\sum_{t=s-p+j-2}^{p} X_{12}^{(t)} X_{21}^{(s+j-2-t)} \\
& =\sum_{t=s-p+j-2}^{s-2} X_{12}^{(t)} X_{21}^{(s+j-2-t)}+X_{12}^{(s-1)} X_{21}^{(j-1)}+\sum_{t=s}^{p} X_{12}^{(t)} X_{21}^{(s+j-2-t)} \\
& =\sum_{m=j}^{p} X_{12}^{(s+j-2-m)} X_{21}^{(m)}+X_{12}^{(s-1)} X_{21}^{(j-1)}+\sum_{t=s}^{p} X_{12}^{(t)} X_{21}^{(s+j-2-t)} \text { (fazendo } m=s+j-2-t \text { ), }
\end{aligned}
$$

\footnotetext{
${ }^{4}$ Como $p-s+3 \leq j \leq p$ e $3 \leq s \leq p$, segue-se $p+1 \leq s+j-2 \leq p+s-2 \leq 2 p$ e portanto, $1 \leq s-p+j-2 \leq p$.
} 
logo da proposição (5.9) ou corolário (5.10),

$$
p_{p s+j-2}(A)=\underbrace{\sum_{m=j}^{p} a_{12}^{(s+j-2-m)} a_{21}^{(m)}}_{=0}+a_{12}^{(s-1)} a_{21}^{(j-1)}+\underbrace{\sum_{t=s}^{p} a_{12}^{(t)} a_{21}^{(s+j-2-t)}}_{=0}=0,
$$

$\operatorname{disso} a_{21}^{(j-1)}=0$ e portanto

$$
A \in \mathfrak{G}_{s}, 3 \leq s \leq p .
$$

\subsubsection{Uma decomposição para $\mathfrak{G}_{1}$}

Note que, como

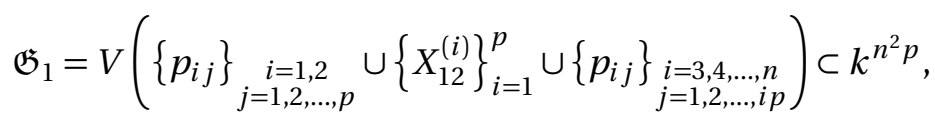

então da proposição (5.9) ou corolário (5.10), pode concluirse que

$$
\mathfrak{G}_{1}=V\left(\mathbf{W} \cup\left\{p_{i j}\right\}_{\substack{i=3,4, \ldots, n \\ j=1,2, \ldots, i p}}\right) \subset k^{n^{2} p}
$$

com

$$
\mathbf{W}:=\bigcup_{i=1}^{p}\left\{X_{11}^{(i)}, X_{12}^{(i)}, X_{22}^{(i)}\right\}
$$

Quando $n=3$, chamaremos $\mathfrak{G}_{1}$ de versão fraca da variedade de Gelfand-Tsetlin $\mathfrak{G}$ para $Y_{p}\left(\mathfrak{g l}_{3}\right)$.

No que segue, iremos procurar uma decomposição (não necessariamente em componentes irredutíveis) para $\mathfrak{G}_{1}$. Para isso precisamos do seguinte lema:

Lema 5.14. Suponha que $p>2$ e $A=\left(a_{i j}^{(t)}\right)_{\substack{i, j=1,2, \ldots, n \\ t=1,2, \ldots, p}} \in \mathfrak{G}_{1}$

1.

(a) $\operatorname{Se} 1 \leq i \leq p-2 e$

$$
a_{13}^{(p)}=a_{13}^{(p-1)}=a_{13}^{(p-2)}=\cdots=a_{13}^{(p-i)}=0,
$$

então

$$
a_{13}^{(p-i-1)}=0 \text { ou } a_{21}^{(p)}=0 \text { ou } a_{32}^{(p)}=0 .
$$

(b) $\operatorname{Se} 1 \leq j \leq i \leq p-2 e$

$$
a_{13}^{(p)}=a_{13}^{(p-1)}=a_{13}^{(p-2)}=\cdots=a_{13}^{(p-i+j)}=a_{21}^{(p)}=a_{21}^{(p-1)}=a_{21}^{(p-2)}=\cdots=a_{21}^{(p-j+1)}=0,
$$

então

$$
a_{13}^{(p-i+j-1)}=0 \text { ou } a_{21}^{(p-j)}=0 \text { ou } a_{32}^{(p)}=0 .
$$

(c) Se $1 \leq i \leq p-2 e$

$$
a_{21}^{(p)}=a_{21}^{(p-1)}=a_{21}^{(p-2)}=\cdots=a_{21}^{(p-i)}=0,
$$

então

$$
a_{13}^{(p)}=0 \text { ou } a_{21}^{(p-i-1)}=0 \text { ou } a_{32}^{(p)}=0 .
$$


2.

(a) Se $1 \leq j \leq i \leq p-2 e$

$$
a_{21}^{(p)}=a_{21}^{(p-1)}=a_{21}^{(p-2)}=\cdots=a_{21}^{(p-i+j)}=a_{32}^{(p)}=a_{32}^{(p-1)}=a_{32}^{(p-2)}=\cdots=a_{32}^{(p-j+1)}=0,
$$

então

$$
a_{13}^{(p)}=0 \text { ou } a_{21}^{(p-i+j-1)}=0 \text { ou } a_{32}^{(p-j)}=0 .
$$

(b) Se $1 \leq i \leq p-2 e$

$$
a_{32}^{(p)}=a_{32}^{(p-1)}=a_{32}^{(p-2)}=\cdots=a_{32}^{(p-i)}=0,
$$

então

$$
a_{13}^{(p)}=0 \text { ou } a_{21}^{(p)}=0 \text { ou } a_{32}^{(p-i-1)}=0 .
$$

3. Se $1 \leq j \leq i \leq p-2 e$

$$
a_{13}^{(p)}=a_{13}^{(p-1)}=a_{13}^{(p-2)}=\cdots=a_{13}^{(p-i+j)}=a_{32}^{(p)}=a_{32}^{(p-1)}=a_{32}^{(p-2)}=\cdots=a_{32}^{(p-j+1)}=0,
$$

então

$$
a_{13}^{(p-i+j-1)}=0 \text { ou } a_{21}^{(p)}=0 \text { ou } a_{32}^{(p-j)}=0 .
$$

Demonstração: Como $1 \leq i \leq p-2$, então $1 \leq p-i-1 \leq p-2$. Podemos considerar o polinômio

$$
p_{33 p-i-1}=\sum_{r=p-i-1}^{p} \sum_{s=2 p-i-1-r}^{p}\left\{-X_{13}^{(r)} X_{22}^{(s)} X_{31}^{(3 p-i-1-r-s)}+X_{12}^{(r)} X_{23}^{(s)} X_{31}^{(3 p-i-1-r-s)}+X_{13}^{(r)} X_{21}^{(s)} X_{32}^{(3 p-i-1-r-s)}\right\},
$$

do fato que $A \in \mathfrak{G}_{1}$ e da proposição (5.9) ou corolário (5.10), temos a equação

$$
\begin{aligned}
p_{33 p-i-1}(A) & =\sum_{r=p-i-1}^{p} \sum_{s=2 p-i-1-r}^{p}\{\underbrace{-a_{13}^{(r)} a_{22}^{(s)} a_{31}^{(3 p-i-1-r-s)}+a_{12}^{(r)} a_{23}^{(s)} a_{31}^{(3 p-i-1-r-s)}}_{=0}+a_{13}^{(r)} a_{21}^{(s)} a_{32}^{(3 p-i-1-r-s)}\} \\
0 & =\sum_{r=p-i-1}^{p} \sum_{s=2 p-i-1-r}^{p} a_{13}^{(r)} a_{21}^{(s)} a_{32}^{(3 p-i-1-r-s)}
\end{aligned}
$$

1.

(a) Se $1 \leq i \leq p-2$ e

$$
a_{13}^{(p)}=a_{13}^{(p-1)}=a_{13}^{(p-2)}=\cdots=a_{13}^{(p-i)}=0,
$$

então

$$
\begin{aligned}
p_{33 p-i-1}(A) & =\sum_{r=p-i-1}^{p} \sum_{s=2 p-i-1-r}^{p} a_{13}^{(r)} a_{21}^{(s)} a_{32}^{(3 p-i-1-r-s)}=0 \\
0 & =a_{13}^{(p-i-1)} a_{21}^{(p)} a_{32}^{(p)}+\underbrace{\sum_{r=p-i}^{p} \sum_{s=2 p-i-1-r}^{p} a_{13}^{(r)} a_{21}^{(s)} a_{32}^{(3 p-i-1-r-s)}}_{=0} \\
0 & =a_{13}^{(p-i-1)} a_{21}^{(p)} a_{32}^{(p)},
\end{aligned}
$$

assim

$$
a_{13}^{(p-i-1)}=0 \text { ou } a_{21}^{(p)}=0 \text { ou } a_{32}^{(p)}=0 .
$$


(b) Se $1 \leq j \leq i \leq p-2 \mathrm{e}$

$$
a_{13}^{(p)}=a_{13}^{(p-1)}=a_{13}^{(p-2)}=\cdots=a_{13}^{(p-i+j)}=a_{21}^{(p)}=a_{21}^{(p-1)}=a_{21}^{(p-2)}=\cdots=a_{21}^{(p-j+1)}=0,
$$

então

$$
\begin{aligned}
p_{33 p-i-1}(A)= & \sum_{r=p-i-1}^{p} \sum_{s=2 p-i-1-r}^{p} a_{13}^{(r)} a_{21}^{(s)} a_{32}^{(3 p-i-1-r-s)}=0 \\
0= & \underbrace{\sum_{r=p-i-1}^{p-i+j-2} \sum_{s=2 p-i-1-r}^{p} a_{13}^{(r)} a_{21}^{(s)} a_{32}^{(3 p-i-1-r-s)}}_{=0}+\sum_{s=p-j}^{p} a_{13}^{(p-i+j-1)} a_{21}^{(s)} a_{32}^{(2 p-j-s)}+ \\
& +\underbrace{\sum_{r=p-i+j}^{p} \sum_{s=2 p-i-1-r}^{p} a_{13}^{(r)} a_{21}^{(s)} a_{32}^{(3 p-i-1-r-s)}}_{=0} \\
0= & \sum_{s=p-j}^{p} a_{13}^{(p-i+j-1)} a_{21}^{(s)} a_{32}^{(2 p-j-s)} \\
0= & a_{13}^{(p-i+j-1)} a_{21}^{(p-j)} a_{32}^{(p)}+\underbrace{\sum_{s=p-j+1}^{p} a_{13}^{(p-i+j-1)} a_{21}^{(s)} a_{32}^{(2 p-j-s)}}_{=0} \\
0= & a_{13}^{(p-i+j-1)} a_{21}^{(p-j)} a_{32}^{(p)},
\end{aligned}
$$

logo

$$
a_{13}^{(p-i+j-1)}=0 \text { ou } a_{21}^{(p-j)}=0 \text { ou } a_{32}^{(p)}=0 .
$$

(c) Se $1 \leq i \leq p-2$ e

$$
a_{21}^{(p)}=a_{21}^{(p-1)}=a_{21}^{(p-2)}=\cdots=a_{21}^{(p-i)}=0,
$$

então

$$
\begin{aligned}
p_{33 p-i-1}(A) & =\sum_{r=p-i-1}^{p} \sum_{s=2 p-i-1-r}^{p} a_{13}^{(r)} a_{21}^{(s)} a_{32}^{(3 p-i-1-r-s)}=0 \\
0 & =\underbrace{\sum_{r=p-i-1}^{p-1} \sum_{s=2 p-i-1-r}^{p} a_{13}^{(r)} a_{21}^{(s)} a_{32}^{(3 p-i-1-r-s)}}_{=0}+\sum_{s=p-i-1}^{p} a_{13}^{(p)} a_{21}^{(s)} a_{32}^{(2 p-i-1-s)} \\
0 & =\sum_{s=p-i-1}^{p} a_{13}^{(p)} a_{21}^{(s)} a_{32}^{(2 p-i-1-s)} \\
0 & =a_{13}^{(p)} a_{21}^{(p-i-1)} a_{32}^{(p)}+\underbrace{\sum_{s=p-i}^{p} a_{13}^{(p)} a_{21}^{(s)} a_{32}^{(2 p-i-1-s)}}_{=0} \\
0 & =a_{13}^{(p)} a_{21}^{(p-i-1)} a_{32}^{(p)}
\end{aligned}
$$

$\log 0$

$$
a_{13}^{(p)}=0 \quad \text { ou } \quad a_{21}^{(p-i-1)}=0 \quad \text { ou } \quad a_{32}^{(p)}=0 .
$$


2.

(a) Se $1 \leq j \leq i \leq p-2$ e

$$
a_{21}^{(p)}=a_{21}^{(p-1)}=a_{21}^{(p-2)}=\cdots=a_{21}^{(p-i+j)}=a_{32}^{(p)}=a_{32}^{(p-1)}=a_{32}^{(p-2)}=\cdots=a_{32}^{(p-j+1)}=0,
$$

então

$$
\begin{aligned}
p_{33 p-i-1}(A)= & \sum_{r=p-i-1}^{p} \sum_{s=2 p-i-1-r}^{p} a_{13}^{(r)} a_{21}^{(s)} a_{32}^{(3 p-i-1-r-s)}=0 \\
0= & \sum_{r=p-i-1}^{p} \sum_{s=2 p-i-1-r}^{p} a_{13}^{(3 p-i-1-r-s)} a_{21}^{(r)} a_{32}^{(s)} \\
0= & \underbrace{\sum_{r=p-i-1}^{p-i+j-2} \sum_{s=2 p-i-1-r}^{p} a_{13}^{(3 p-i-1-r-s)} a_{21}^{(r)} a_{32}^{(s)}}_{=0}+\sum_{s=p-j}^{p} a_{13}^{(2 p-j-s)} a_{21}^{(p-i+j-1)} a_{32}^{(s)} \\
& +\underbrace{\sum_{r=p-i+j}^{p} \sum_{s=2 p-i-1-r}^{p} a_{13}^{(3 p-i-1-r-s)} a_{21}^{(r)} a_{32}^{(s)}}_{=0} \\
0= & \sum_{s=p-j}^{\sum^{p} a_{13}^{(2 p-j-s)} a_{21}^{(p-i+j-1)} a_{32}^{(s)}} \\
0= & a_{13}^{(p)} a_{21}^{(p-i+j-1)} a_{32}^{(p-j)}+\underbrace{\sum_{s=p-j+1}^{p} a_{13}^{(2 p-j-s)} a_{21}^{(p-i+j-1)} a_{32}^{(s)}}_{=0} \\
0= & a_{13}^{(p)} a_{21}^{(p-i+j-1)} a_{32}^{(p-j)},
\end{aligned}
$$

logo

$$
a_{13}^{(p)}=0 \quad \text { ou } a_{21}^{(p-i+j-1)}=0 \text { ou } a_{32}^{(p-j)}=0 .
$$

(b) Se $1 \leq i \leq p-2$ e

$$
a_{32}^{(p)}=a_{32}^{(p-1)}=a_{32}^{(p-2)}=\cdots=a_{32}^{(p-i)}=0,
$$

então

$$
\begin{aligned}
p_{33 p-i-1}(A) & =\sum_{r=p-i-1}^{p} \sum_{s=2 p-i-1-r}^{p} a_{13}^{(r)} a_{21}^{(s)} a_{32}^{(3 p-i-1-r-s)}=0 \\
0 & =\sum_{r=p-i-1}^{p} \sum_{s=2 p-i-1-r}^{p} a_{13}^{(3 p-i-1-r-s)} a_{21}^{(r)} a_{32}^{(s)} \\
0 & =\underbrace{\sum_{r=p-i-1}^{p-1} \sum_{s=2 p-i-1-r}^{p} a_{13}^{(3 p-i-1-r-s)} a_{21}^{(r)} a_{32}^{(s)}}_{=0}+\sum_{s=p-i-1}^{p} a_{13}^{(2 p-i-1-s)} a_{21}^{(p)} a_{32}^{(s)} \\
0 & =\sum_{s=p-i-1}^{\sum_{13}^{p}} a_{13}^{(2 p-i-1-s)} a_{21}^{(p)} a_{32}^{(s)} \\
0 & =a_{13}^{(p)} a_{21}^{(p)} a_{32}^{(p-i-1)}+\underbrace{\sum_{s=p-i}^{p} a_{13}^{(2 p-i-1-s)} a_{21}^{(p)} a_{32}^{(s)}}_{=0}
\end{aligned}
$$




$$
0=a_{13}^{(p)} a_{21}^{(p)} a_{32}^{(p-i-1)}
$$

assim

$$
a_{13}^{(p)}=0 \quad \text { ou } \quad a_{21}^{(p)}=0 \quad \text { ou } \quad a_{32}^{(p-i-1)}=0 .
$$

3. Se $1 \leq j \leq i \leq p-2 \mathrm{e}$

$$
a_{13}^{(p)}=a_{13}^{(p-1)}=a_{13}^{(p-2)}=\cdots=a_{13}^{(p-i+j)}=a_{32}^{(p)}=a_{32}^{(p-1)}=a_{32}^{(p-2)}=\cdots=a_{32}^{(p-j+1)}=0,
$$

então

$$
\begin{aligned}
p_{33 p-i-1}(A)= & \sum_{r=p-i-1}^{p} \sum_{s=2 p-i-1-r}^{p} a_{13}^{(r)} a_{21}^{(s)} a_{32}^{(3 p-i-1-r-s)}=0 \\
0= & \sum_{r=p-i-1}^{p} \sum_{s=2 p-i-1-r}^{p} a_{13}^{(r)} a_{21}^{(3 p-i-1-r-s)} a_{32}^{(s)} \\
0= & \underbrace{\sum_{r=p-i-1}^{p-i+j-2} \sum_{s=2 p-i-1-r}^{p} a_{13}^{(r)} a_{21}^{(3 p-i-1-r-s)} a_{32}^{(s)}}_{=0}+\sum_{s=p-j}^{p} a_{13}^{(p-i+j-1)} a_{21}^{(2 p-j-s)} a_{32}^{(s)}+ \\
& +\underbrace{\sum_{r=p-i+j}^{p} \sum_{s=2 p-i-1-r}^{p} a_{13}^{(r)} a_{21}^{(3 p-i-1-r-s)} a_{32}^{(s)}}_{=0} \\
0= & \sum_{s=p-j}^{p} a_{13}^{(p-i+j-1)} a_{21}^{(2 p-j-s)} a_{32}^{(s)} \\
0= & a_{13}^{(p-i+j-1)} a_{21}^{(p)} a_{32}^{(p-j)}+\underbrace{\sum_{s=p-j+1}^{p} a_{13}^{(p-i+j-1)} a_{21}^{(2 p-j-s)} a_{32}^{(s)}}_{=0} \\
0= & a_{13}^{(p-i+j-1)} a_{21}^{(p)} a_{32}^{(p-j)},
\end{aligned}
$$

logo

$$
a_{13}^{(p-i+j-1)}=0 \quad \text { ou } \quad a_{21}^{(p)}=0 \text { ou } a_{32}^{(p-j)}=0 .
$$

Notação 5.15. Para $Y_{p}\left(\mathfrak{g l}_{n}\right)$ usaremos as seguintes subvariedades de $\mathfrak{G}_{1}$ :

1

$$
\mathbb{W}^{p}:=V\left(\boldsymbol{W} \cup\left\{p_{3 j}\right\}_{j=1}^{3 p-3} \cup\left\{X_{32}^{(p)}, X_{21}^{(p)}, X_{13}^{(p)}\right\} \cup\left\{p_{i j}\right\} \begin{array}{c}
i=4,5, \ldots, n \\
j=1,2, \ldots, i p
\end{array}\right)
$$

2.

$$
\mathbb{W}_{11}:=V\left(\boldsymbol{W} \cup\left\{p_{3 j}\right\}_{j=1}^{2 p} \cup\left\{X_{13}^{(t)}\right\}_{t=1}^{p} \cup\left\{p_{i j}\right\}_{\substack{i=4,5, \ldots, n \\ j=1,2, \ldots, i p}}\right)
$$

3. Para cada $s=2,3, \ldots, p$,

$$
\mathbb{W}_{1 s}:=V\left(\boldsymbol{W} \cup\left\{p_{3 j}\right\}_{j=1}^{2 p} \cup\left\{X_{13}^{(t)}\right\}_{t=s}^{p} \cup\left\{X_{21}^{(t)}\right\}_{t=p-(s-2)}^{p} \cup\left\{p_{i j}\right\}_{\substack{i=4,5, \ldots, n \\ j=1,2, \ldots, i p}}\right) .
$$

4.

$$
\mathbb{W}_{1 p+1}:=V\left(\boldsymbol{W} \cup\left\{p_{3 j}\right\}_{j=1}^{2 p} \cup\left\{X_{21}^{(t)}\right\}_{t=1}^{p} \cup\left\{p_{i j}\right\}_{\substack{i=4,5, \ldots, n \\ j=1,2, \ldots, i p}}\right)
$$


5.

$$
\mathbb{W}_{1}:=\bigcup_{s=1}^{p+1} \mathbb{W}_{1 s}
$$

6. Para cada $s=2,3, \ldots, p$,

$$
\mathbb{W}_{2 s}:=V\left(\boldsymbol{W} \cup\left\{p_{3 j}\right\}_{j=1}^{2 p} \cup\left\{X_{21}^{(t)}\right\}_{t=s}^{p} \cup\left\{X_{32}^{(t)}\right\}_{t=p-(s-2)}^{p} \cup\left\{p_{i j}\right\} \begin{array}{c}
i=4,5, \ldots, n \\
j=1,2, \ldots, i p
\end{array}\right) .
$$

7.

$$
\mathbb{W}_{2 p+1}:=V\left(\boldsymbol{W} \cup\left\{p_{3 j}\right\}_{j=1}^{2 p} \cup\left\{X_{32}^{(t)}\right\}_{t=1}^{p} \cup\left\{p_{i j}\right\} \begin{array}{c}
i=4,5, \ldots, n \\
j=1,2, \ldots, i p
\end{array}\right)
$$

8.

$$
\mathbb{W}_{2}:=\bigcup_{s=2}^{p+1} \mathbb{W}_{2 s}
$$

9. Para cada $s=2,3, \ldots, p$,

$$
\mathbb{W}_{3 s}:=V\left(\boldsymbol{W} \cup\left\{p_{3 j}\right\}_{j=1}^{2 p} \cup\left\{X_{13}^{(t)}\right\}_{t=s}^{p} \cup\left\{X_{32}^{(t)}\right\}_{t=p-(s-2)}^{p} \cup\left\{p_{i j}\right\} \underset{\substack{i=4,5, \ldots, n \\ j=1,2, \ldots, i p}}{j}\right) .
$$

10.

$$
\mathbb{W}_{3}:=\bigcup_{s=2}^{p} \mathbb{W}_{3 s}
$$

Proposição 5.16. Para $Y_{p}\left(\mathfrak{g l}_{n}\right)$

$$
\begin{gathered}
\mathfrak{G}_{1}=\mathbb{W}_{1} \cup \mathbb{W}_{2} \text {, se } p=1 ; \\
\mathfrak{G}_{1}=\mathbb{W}_{1} \cup \mathbb{W}_{2} \cup \mathbb{W}_{3} \text {, se } p=2 ; \\
\mathfrak{G}_{1}=\mathbb{W}^{p} \cup \mathbb{W}_{1} \cup \mathbb{W}_{2} \cup \mathbb{W}_{3} \text {, se } p \geq 3 .
\end{gathered}
$$

Demonstração: Claramente, cada $\mathbb{W}$ 's está contido em $\mathfrak{G}_{1}$, então só temos que provar que

$$
\begin{gathered}
\mathfrak{G}_{1} \subseteq \mathbb{W}_{1} \cup \mathbb{W}_{2} \text {, para } p=1, \\
\mathfrak{G}_{1} \subseteq \mathbb{W}_{1} \cup \mathbb{W}_{2} \cup \mathbb{W}_{3} \text {, para } p=2, \\
\mathfrak{G}_{1} \subseteq \mathbb{W}^{p} \cup \mathbb{W}_{1} \cup \mathbb{W}_{2} \cup \mathbb{W}_{3} \text {, para } p \geq 3 .
\end{gathered}
$$

De fato, consideremos $A=\left(a_{i j}^{(t)}\right)_{\substack{i, j=1,2, \ldots, n \\ t=1,2, \ldots, p}} \in \mathfrak{G}_{1}$. Para esta prova usaremos os polinômios

$$
p_{32 p+i}=\sum_{r=i}^{p} \sum_{s=p+i-r}^{p}\left\{-X_{13}^{(r)} X_{22}^{(s)} X_{31}^{(2 p+i-r-s)}+X_{12}^{(r)} X_{23}^{(s)} X_{31}^{(2 p+i-r-s)}+X_{13}^{(r)} X_{21}^{(s)} X_{32}^{(2 p+i-r-s)}\right\}, \quad i=p-1, p .
$$

Comecemos com o polinômio

$$
p_{33 p}=-X_{13}^{(p)} X_{22}^{(p)} X_{31}^{(p)}+X_{12}^{(p)} X_{23}^{(p)} X_{31}^{(p)}+X_{13}^{(p)} X_{21}^{(p)} X_{32}^{(p)},
$$

com isso e a proposição (5.9) ou corolário (5.10),

$$
p_{33 p}(A)=-\underbrace{a_{13}^{(p)} a_{22}^{(p)} a_{31}^{(p)}+a_{12}^{(p)} a_{23}^{(p)} a_{31}^{(p)}}_{=0}+a_{13}^{(p)} a_{21}^{(p)} a_{32}^{(p)}=0,
$$


logo

$$
a_{13}^{(p)}=0 \quad \text { ou } \quad a_{21}^{(p)}=0 \quad \text { ou } \quad a_{32}^{(p)}=0,
$$

como consequência disso, se $p=1$ então $A \in \mathbb{W}_{1} \cup \mathbb{W}_{2}$. Para $p>1$, consideremos o polinômio

$$
p_{33 p-1}=\sum_{r=p-1}^{p} \sum_{s=2 p-1-r}^{p}\left\{-X_{13}^{(r)} X_{22}^{(s)} X_{31}^{(3 p-1-r-s)}+X_{12}^{(r)} X_{23}^{(s)} X_{31}^{(3 p-1-r-s)}+X_{13}^{(r)} X_{21}^{(s)} X_{32}^{(3 p-1-r-s)}\right\},
$$

segue da proposição (5.9) ou corolário (5.10) que

$$
\begin{aligned}
p_{33 p-1}(A) & =\sum_{r=p-1}^{p} \sum_{s=2 p-1-r}^{p}\{\underbrace{-a_{13}^{(r)} a_{22}^{(s)} a_{31}^{(3 p-1-r-s)}+a_{12}^{(r)} a_{23}^{(s)} a_{31}^{(3 p-1-r-s)}}_{=0}+a_{13}^{(r)} a_{21}^{(s)} a_{32}^{(3 p-1-r-s)}\}=0 \\
& =\sum_{r=p-1}^{p} \sum_{s=2 p-1-r}^{p} a_{13}^{(r)} a_{21}^{(s)} a_{32}^{(3 p-1-r-s)}=0 \\
& =\sum_{s=p}^{p} a_{13}^{(p-1)} a_{21}^{(s)} a_{32}^{(2 p-s)}+\sum_{s=p-1}^{p} a_{13}^{(p)} a_{21}^{(s)} a_{32}^{(2 p-1-s)}=0 \\
& =a_{13}^{(p-1)} a_{21}^{(p)} a_{32}^{(p)}+a_{13}^{(p)} a_{21}^{(p-1)} a_{32}^{(p)}+a_{13}^{(p)} a_{21}^{(p)} a_{32}^{(p-1)}=0,
\end{aligned}
$$

assim

$$
a_{13}^{(p)}=a_{13}^{(p-1)}=0 \text { ou } a_{13}^{(p)}=a_{21}^{(p)}=0 \text { ou } a_{13}^{(p)}=a_{32}^{(p)}=0
$$

ou

$$
a_{21}^{(p)}=a_{21}^{(p-1)}=0 \text { ou } a_{21}^{(p)}=a_{32}^{(p)}=0
$$

ou

$$
a_{32}^{(p)}=a_{32}^{(p-1)}=0,
$$

portanto, no caso que $p=2$ tem-se $A \in \mathbb{W}_{1} \cup \mathbb{W}_{2} \cup \mathbb{W}_{3}$.

Agora, se $p>2$ pelo lema (5.14) temos os casos:

$$
a_{13}^{(p)}=a_{13}^{(p-1)}=a_{13}^{(p-2)}=0 \text { ou } a_{13}^{(p)}=a_{13}^{(p-1)}=a_{21}^{(p)}=0 \text { ou } a_{13}^{(p)}=a_{13}^{(p-1)}=a_{32}^{(p)}=0
$$

ou

$$
a_{13}^{(p)}=a_{21}^{(p)}=a_{21}^{(p-1)}=0 \text { ou } a_{13}^{(p)}=a_{21}^{(p)}=a_{32}^{(p)}=0
$$

ou

$$
a_{13}^{(p)}=a_{32}^{(p)}=a_{32}^{(p-1)}=0
$$

ou

$$
a_{21}^{(p)}=a_{21}^{(p-1)}=a_{21}^{(p-2)}=0 \text { ou } a_{21}^{(p)}=a_{21}^{(p-1)}=a_{32}^{(p)}=0
$$

ou

$$
a_{21}^{(p)}=a_{32}^{(p)}=a_{32}^{(p-1)}=0
$$

ou

$$
a_{32}^{(p)}=a_{32}^{(p-1)}=a_{32}^{(p-2)}=0
$$

Quando

$$
a_{13}^{(p)}=a_{21}^{(p)}=a_{32}^{(p)}=0,
$$

temos que $A \in \mathbb{W}^{p}$ e portanto podemos assumir que 
Similarmente, quando

$$
a_{13}^{(t)}=0, \text { para todo } t=1,2, \ldots, p
$$

ou

$$
a_{21}^{(t)}=0, \text { para todo } t=1,2, \ldots, p
$$

$\mathrm{ou}$

$$
a_{32}^{(t)}=0, \text { para todo } t=1,2, \ldots, p
$$

temos que $A \in \mathbb{W}_{11} \cup \mathbb{W}_{1 p+1} \cup \mathbb{W}_{2 p+1}$ e portanto podemos assumir que

$$
A \notin \mathbb{W}_{11} \cup \mathbb{W}_{1 p+1} \cup \mathbb{W}_{2 p+1} .
$$

Continuando com este raciocinio, suponha que

$$
A \notin \mathbb{W}^{p} \cup \mathbb{W}_{1} \cup \mathbb{W}_{2}
$$

e provemos que

$$
A \in \mathbb{W}_{3} .
$$

Claramente, existe $s \in\{2,3, \ldots, p\}$ tal que

$$
a_{13}^{(t)}=0, \text { para cada } t=s, s+1, \ldots, p \text { e } a_{13}^{(s-1)} \neq 0
$$

pois $A \notin \mathbb{W}_{11}$, segue do lema (5.14) item (1.a) que

$$
a_{21}^{(p)}=0 \text { ou } a_{32}^{(p)}=0 .
$$

Notemos que, se $a_{21}^{(p)}=0$ existe $j \in\{p-(s-3), p-(s-4), \ldots, p\}$ tal que

$$
a_{21}^{(t)}=0, \text { para cada } t=j, j+1, \ldots, p \text { e } a_{21}^{(j-1)} \neq 0
$$

pois $A \notin \mathbb{W}_{1 s}$ e como consequência do lema (5.14) item (1.b), temos

$$
a_{32}^{(p)}=0
$$

o qual contradiz o fato que $A \notin \mathbb{W}^{p}$. Portanto

$$
a_{21}^{(p)} \neq 0 \text { e } a_{32}^{(p)}=0 .
$$

Novamente, pelo lema (5.14) item (3)

$$
a_{32}^{(p-1)}=0
$$

e continuando desta forma temos que

$$
A \in \mathbb{W}_{3 s} \subseteq \mathbb{W}_{3} .
$$

Agora o que vamos fazer, é melhorar a decomposição da proposição (5.16). Com este objetivo, iremos precisar do seguinte lema:

Lema 5.17. Suponha que $p>2$ e $A=\left(a_{i j}^{(t)}\right)_{\substack{i, j=1,2, \ldots, n \\ t=1,2, \ldots, p}} \in \mathfrak{G}_{1}$.

Se $2 \leq s \leq p-1,0 \leq j \leq p-s e$

$$
\begin{gathered}
a_{13}^{(p)}=a_{13}^{(p-1)}=a_{13}^{(p-2)}=\cdots=a_{13}^{(s+1)}=a_{13}^{(s)}=0, \\
a_{32}^{(p)}=a_{32}^{(p-1)}=a_{32}^{(p-2)}=\cdots=a_{32}^{(p-(s-2))}=a_{32}^{(p-(s-2)-1)}=a_{32}^{(p-(s-2)-2)}=\cdots=a_{32}^{(p-(s-2)-j)}=0,
\end{gathered}
$$


então

$$
a_{13}^{(s-1)}=0 \text { ou } a_{21}^{(p)}=0 \text { ou } a_{32}^{(p-(s-2)-j-1)}=0 .
$$

Demonstração: Consideremos os polinômios ${ }^{5}$

$$
\begin{aligned}
p_{32 p-j}= & -\sum_{t=p-j}^{p} X_{23}^{(t)} X_{32}^{(2 p-j-t)}-\sum_{t=p-j}^{p} X_{13}^{(t)} X_{31}^{(2 p-j-t)}+ \\
& +\sum_{r=1}^{p-j-1} \sum_{t=p-j-r}^{p}\left\{-X_{13}^{(r)} X_{22}^{(t)} X_{31}^{(2 p-j-r-t)}+X_{12}^{(r)} X_{23}^{(t)} X_{31}^{(2 p-j-r-t)}+X_{13}^{(r)} X_{21}^{(t)} X_{32}^{(2 p-j-r-t)}\right\}+ \\
& +\sum_{r=p-j}^{p} \sum_{t=1}^{2 p-j-r-1}\left\{-X_{13}^{(r)} X_{22}^{(t)} X_{31}^{(2 p-j-r-t)}+X_{12}^{(r)} X_{23}^{(t)} X_{31}^{(2 p-j-r-t)}+X_{13}^{(r)} X_{21}^{(t)} X_{32}^{(2 p-j-r-t)}\right\},
\end{aligned}
$$

do fato que $A \in \mathfrak{G}_{1}$ temos a equação ${ }^{6}$

$$
\begin{aligned}
p_{32 p-j}(A)= & -\underbrace{\sum_{t=p-j}^{p} a_{23}^{(t)} a_{32}^{(2 p-j-t)}}_{=0}-\underbrace{\sum_{t=p-j}^{p} a_{13}^{(t)} a_{31}^{(2 p-j-t)}}_{=0}+ \\
& +\sum_{r=1}^{p-j-1} \sum_{t=p-j-r}^{p}\{\underbrace{-a_{13}^{(r)} a_{22}^{(t)} a_{31}^{(2 p-j-r-t)}+a_{12}^{(r)} a_{23}^{(t)} a_{31}^{(2 p-j-r-t)}}_{=0}+a_{13}^{(r)} a_{21}^{(t)} a_{32}^{(2 p-j-r-t)}\}+ \\
& +\sum_{r=p-j}^{p} \sum_{t=1}^{2 p-j-r-1}\{\underbrace{-a_{13}^{(r)} a_{22}^{(t)} a_{31}^{(2 p-j-r-t)}+a_{12}^{(r)} a_{23}^{(t)} a_{31}^{(2 p-j-r-t)}}_{=0}+\underbrace{a_{13}^{(r)} a_{21}^{(t)} a_{32}^{(2 p-j-r-t)}}_{=0}\}=0 \\
= & \sum_{r=1}^{p-j-1} \sum_{t=p-j-r}^{p} a_{13}^{(r)} a_{21}^{(t)} a_{32}^{(2 p-j-r-t)}=0 .
\end{aligned}
$$

Se $s=2$

$$
\begin{aligned}
p_{32 p-j}(A) & =\sum_{r=1}^{p-j-1} \sum_{t=p-j-r}^{p} a_{13}^{(r)} a_{21}^{(t)} a_{32}^{(2 p-j-r-t)}=0 \\
& =\sum_{t=p-j-1}^{p} a_{13}^{(1)} a_{21}^{(t)} a_{32}^{(2 p-j-1-t)}+\underbrace{\sum_{r=2}^{p-j-1} \sum_{t=p-j-r}^{p} a_{13}^{(r)} a_{21}^{(t)} a_{32}^{(2 p-j-r-t)}=0}_{=0} \\
& =\sum_{t=p-j-1}^{p} a_{13}^{(1)} a_{21}^{(t)} a_{32}^{(2 p-j-1-t)}=0 .
\end{aligned}
$$

Se $2<s<p-j$

$$
\begin{aligned}
p_{32 p-j}(A)= & \sum_{r=1}^{s-2} \sum_{t=p-j-r}^{p} a_{13}^{(r)} a_{21}^{(t)} a_{32}^{(2 p-j-r-t)}+\sum_{t=p-j-s+1}^{p} a_{13}^{(s-1)} a_{21}^{(t)} a_{32}^{(2 p-j-s+1-t)}+ \\
& +\underbrace{\sum_{r=s}^{p-j-1} \sum_{t=p-j-r}^{p} a_{13}^{(r)} a_{21}^{(t)} a_{32}^{(2 p-j-r-t)}}_{=0}=0
\end{aligned}
$$

${ }^{5}$ Note que $s \leq p-j$.

${ }^{6}$ Aqui usamos o fato que $s \leq p-j$ e $p-(s-2)-j \leq p-j$. 


$$
\begin{aligned}
& =\sum_{r=1}^{s-2} \sum_{t=p-j-r}^{p} a_{13}^{(r)} a_{21}^{(t)} a_{32}^{(2 p-j-r-t)}+\sum_{t=p-j-s+1}^{p} a_{13}^{(s-1)} a_{21}^{(t)} a_{32}^{(2 p-j-s+1-t)}=0 \\
& =\underbrace{\sum_{r=1}^{s-2} \sum_{m=p-j-r}^{p} a_{13}^{(r)} a_{21}^{(2 p-j-r-m)} a_{32}^{(m)}}_{=0}+\sum_{t=p-j-s+1}^{p} a_{13}^{(s-1)} a_{21}^{(t)} a_{32}^{(2 p-j-s+1-t)}=0, \\
& =\sum_{t=p-j-s+1}^{p} a_{13}^{(s-1)} a_{21}^{(t)} a_{32}^{(2 p-j-s+1-t)}=0,
\end{aligned}
$$

onde na passagem da linha três para a linha quatro fizemos $m=2 p-j-r-t$.

Se $s=p-j$

$$
\begin{aligned}
p_{32 p-j}(A) & =\sum_{r=1}^{p-j-1} \sum_{t=p-j-r}^{p} a_{13}^{(r)} a_{21}^{(t)} a_{32}^{(2 p-j-r-t)}=0 \\
& =\sum_{r=1}^{p-j-2} \sum_{t=p-j-r}^{p} a_{13}^{(r)} a_{21}^{(t)} a_{32}^{(2 p-j-r-t)}+\sum_{t=1}^{p} a_{13}^{(p-j-1)} a_{21}^{(t)} a_{32}^{(p+1-t)}=0 \\
& =\underbrace{\sum_{r=1}^{p-j-2} \sum_{m=p-j-r}^{p} a_{13}^{(r)} a_{21}^{(2 p-j-r-m)} a_{32}^{(m)}}_{=0}+\sum_{t=1}^{p} a_{13}^{(p-j-1)} a_{21}^{(t)} a_{32}^{(p+1-t)}=0 \\
& =\sum_{t=1}^{p} a_{13}^{(p-j-1)} a_{21}^{(t)} a_{32}^{(p+1-t)}=0,
\end{aligned}
$$

onde na passagem da linha dois para a linha três fizemos $m=2 p-j-r-t$.

Agora, por todos os casos anteriores temos

$$
\begin{aligned}
p_{32 p-j}(A) & =\sum_{t=p-j-s+1}^{p} a_{13}^{(s-1)} a_{21}^{(t)} a_{32}^{(2 p-j-s+1-t)}=0 \\
& =\sum_{t=p-j-s+1}^{p-1} a_{13}^{(s-1)} a_{21}^{(t)} a_{32}^{(2 p-j-s+1-t)}+a_{13}^{(s-1)} a_{21}^{(p)} a_{32}^{(p-j-s+1)}=0 \\
& =\underbrace{\sum_{m=p-j-s+2}^{p} a_{13}^{(s-1)} a_{21}^{(2 p-j-s+1-m)} a_{32}^{(m)}}_{=0}+a_{13}^{(s-1)} a_{21}^{(p)} a_{32}^{(p-j-s+1)}=0 \\
& =a_{13}^{(s-1)} a_{21}^{(p)} a_{32}^{(p-j-s+1)}=0 .
\end{aligned}
$$

onde na passagem da linha dois para a linha três fizemos $m=2 p-j-s+1-t$.

Proposição 5.18. Para $Y_{p}\left(\mathfrak{g l}_{n}\right) \operatorname{com} p>1$

$$
\mathbb{W}_{3} \subseteq \mathbb{W}^{p} \cup \mathbb{W}_{1} \cup \mathbb{W}_{2}
$$

Demonstração: Suponha $s \in\{2,3, \ldots, p\}$ e $A=\left(a_{i j}^{(t)}\right)_{\substack{i, j=1,2, \ldots, n \\ t=1,2, \ldots, p}} \in \mathbb{W}_{3 s}$, isto implica que

$$
\begin{gathered}
a_{13}^{(p)}=a_{13}^{(p-1)}=\cdots=a_{13}^{(s)}=0, \\
a_{32}^{(p)}=a_{32}^{(p-1)}=\cdots=a_{32}^{(p-(s-2))}=0 .
\end{gathered}
$$


Pelo lema (5.17)

$$
a_{13}^{(s-1)}=0 \text { ou } a_{21}^{(p)}=0 \text { ou } a_{32}^{(p-(s-2)-1)}=0 .
$$

Se $a_{21}^{(p)}=0$, então $A \in \mathbb{W}^{p}$.

Se $a_{13}^{(t)}=0 \forall t=1,2, \ldots, p$, então $A \in \mathbb{W}_{11} \subseteq \mathbb{W}_{1}$.

Se existe $t \in\{2,3, \ldots, s\}$ tal que

$$
a_{13}^{(j)}=0 \forall j=t, t+1, \ldots, p \text { e } a_{13}^{(t-1)} \neq 0,
$$

então do lema (5.17)

$$
A \in \mathbb{W}_{2 p+1} \subseteq \mathbb{W}_{2}
$$

Corolário 5.19. Para $Y_{p}\left(\mathfrak{g l}_{n}\right)$

$$
\begin{gathered}
\mathfrak{G}_{1}=\mathbb{W}_{1} \cup \mathbb{W}_{2} \text {, se } p=1,2 ; \\
\mathfrak{G}_{1}=\mathbb{W}^{p} \cup \mathbb{W}_{1} \cup \mathbb{W}_{2} \text {, se } p \geq 3 .
\end{gathered}
$$

Demonstração: Segue-se das proposições (5.16) e (5.18).

\subsection{Equidimensionalidade da versão fraca $\mathfrak{G}_{1}$ para $Y_{p}\left(\mathfrak{g l}_{3}\right)$}

Nesta seção demonstraremos que a versão fraca $\mathfrak{G}_{1}$ de $\mathfrak{G}$ para $Y_{p}\left(\mathfrak{g l}_{3}\right)$ é equidimensional com

$$
\operatorname{dim} \mathfrak{G}_{1}=3 p
$$

provando a equidimensionalidade de cada subvariedade $\mathbb{W}$ 's da decomposição do corolário (5.19). Con este objetivo o seguinte teorema de Futorny, Molev e Ovsienko será muito útil.

Teorema 5.20. A variedade de Gelfand-Tsetlin $\mathfrak{G}$ para $Y_{p}\left(\mathfrak{g l}_{2}\right)$ é equidimensional com dimensão $p$.

Demonstração: Ver [Futorny et al. (2005)].

Proposição 5.21. Para todo $s=1,2, \ldots, p, p+1 \operatorname{com} p>2$, a subvariedade $\mathbb{W}_{1 s}$ da versão fraca $\mathfrak{G}_{1}$ da variedade de Gelfand-Tsetlin para $Y_{p}\left(\mathfrak{g l}_{3}\right)$ é equidimensional com dimensão $3 p$.

Demonstração: Usando a notação (5.15) as subvariedades $\mathbb{W}_{1 s}$ 's são:

$$
\mathbb{W}_{1 s}=V\left(\mathbf{X}_{s} \cup\left\{p_{3 j}\right\}_{j=1}^{2 p}\right) \subset k^{9 p}, \quad s=1,2, \ldots, p, p+1,
$$

em que

$$
\begin{aligned}
\mathbf{X}_{1} & =\left\{X_{11}^{(i)}\right\}_{i=1}^{p} \cup\left\{X_{12}^{(i)}\right\}_{i=1}^{p} \cup\left\{X_{22}^{(i)}\right\}_{i=1}^{p} \cup\left\{X_{13}^{(i)}\right\}_{i=1}^{p} \\
\mathbf{X}_{s} & =\left\{X_{11}^{(i)}\right\}_{i=1}^{p} \cup\left\{X_{12}^{(i)}\right\}_{i=1}^{p} \cup\left\{X_{22}^{(i)}\right\}_{i=1}^{p} \cup\left\{X_{13}^{(i)}\right\}_{i=s}^{p} \cup\left\{X_{21}^{(i)}\right\}_{i=p-(s-2)}^{p}, s=2,3, \ldots, p \\
\mathbf{X}_{p+1} & =\left\{X_{11}^{(i)}\right\}_{i=1}^{p} \cup\left\{X_{12}^{(i)}\right\}_{i=1}^{p} \cup\left\{X_{22}^{(i)}\right\}_{i=1}^{p} \cup\left\{X_{21}^{(i)}\right\}_{i=1}^{p} .
\end{aligned}
$$

Provaremos que cada $\mathbb{W}_{1 s}$ é equidimensional com

$$
\operatorname{dim}\left(\mathbb{W}_{1 s}\right)=9 p-(\underbrace{4 p}_{=\left|\mathbf{X}_{s}\right|}+2 p)=3 p .
$$


Para isso, pelo corolário (2.67) e a proposição (2.69), é suficiente provar que

$$
V\left(\mathbf{X}_{s} \cup\left\{p_{3 j}\right\}_{j=1}^{2 p} \cup \mathbf{Y}_{s}\right) \subset k^{9 p}
$$

com

$$
\begin{aligned}
\mathbf{Y}_{1} & =\left\{X_{21}^{(i)}\right\}_{i=1}^{p} \cup\left\{X_{31}^{(i)}\right\}_{i=1}^{p} \\
\mathbf{Y}_{s} & =\left\{X_{13}^{(i)}\right\}_{i=1}^{s-1} \cup\left\{X_{21}^{(i)}\right\}_{i=1}^{p-(s-2)-1} \cup\left\{X_{31}^{(i)}\right\}_{i=1}^{p}, s=2,3, \ldots, p \\
\mathbf{Y}_{p+1} & =\left\{X_{13}^{(i)}\right\}_{i=1}^{p} \cup\left\{X_{31}^{(i)}\right\}_{i=1}^{p}
\end{aligned}
$$

é equidimensional com dimensão $9 p-(\underbrace{4 p}_{=\left|\mathbf{X}_{s}\right|}+2 p+\underbrace{2 p}_{=\left|\mathbf{Y}_{s}\right|})=p$. Para cada $s=1,2, \ldots, p, p+1$

$$
V\left(\mathbf{X}_{s} \cup\left\{p_{3 j}\right\}_{j=1,}^{2 p} \cup \mathbf{Y}_{s}\right)=V\left(\mathbf{Z} \cup\left\{p_{3 j}^{\mathbf{Z}}\right\}_{j=1}^{2 p}\right) \subset k^{9 p},
$$

em que, pela proposição (5.9) e a notação (3.17)

$$
\begin{aligned}
& \mathbf{Z}=\mathbf{X}_{s} \cup \mathbf{Y}_{s}=\left\{X_{11}^{(i)}\right\}_{i=1}^{p} \cup\left\{X_{12}^{(i)}\right\}_{i=1}^{p} \cup\left\{X_{22}^{(i)}\right\}_{i=1}^{p} \cup\left\{X_{13}^{(i)}\right\}_{i=1}^{p} \cup\left\{X_{21}^{(i)}\right\}_{i=1}^{p} \cup\left\{X_{31}^{(i)}\right\}_{i=1}^{p}, \\
& p_{31}^{\mathbf{Z}}=X_{33}^{(1)} \text {, } \\
& p_{32}^{\mathbf{Z}}=X_{33}^{(2)}-X_{23}^{(1)} X_{32}^{(1)}-\underbrace{X_{13}^{(1)} X_{31}^{(1)}}_{=0} \\
& =X_{33}^{(2)}-X_{23}^{(1)} X_{32}^{(1)} \text {, } \\
& p_{33}^{\mathbf{Z}}=X_{33}^{(3)}-X_{23}^{(1)} X_{32}^{(2)}-X_{23}^{(2)} X_{32}^{(1)} \underbrace{-X_{13}^{(1)} X_{31}^{(2)}-X_{13}^{(2)} X_{31}^{(1)}+X_{12}^{(1)} X_{23}^{(1)} X_{31}^{(1)}+X_{13}^{(1)} X_{21}^{(1)} X_{32}^{(1)}}_{=0} \\
& =X_{33}^{(3)}-X_{23}^{(1)} X_{32}^{(2)}-X_{23}^{(2)} X_{32}^{(1)} \text {, } \\
& p_{3 i}^{\mathbf{Z}}=-\sum_{r=1}^{i-2} \sum_{s=2}^{i-r-1} \underbrace{X_{13}^{(r)} X_{22}^{(s)} X_{31}^{(i-r-s)}}_{=0}+X_{33}^{(i)}-\sum_{s=1}^{i-1}(\underbrace{X_{13}^{(s)} X_{31}^{(i-s)}}_{=0}+X_{23}^{(s)} X_{32}^{(i-s)})+ \\
& +\sum_{r=1}^{i-2} \sum_{s=1}^{i-r-1}\{\underbrace{X_{12}^{(r)} X_{23}^{(s)} X_{31}^{(i-r-s)}+X_{13}^{(r)} X_{21}^{(s)} X_{32}^{(i-r-s)}}_{=0}\} \\
& =X_{33}^{(i)}-\sum_{s=1}^{i-1} X_{23}^{(s)} X_{32}^{(i-s)}, \quad i=4,5, \ldots, p, \\
& p_{3 p+1}^{\mathbf{Z}}=\sum_{r=1}^{p-1} \sum_{s=1}^{p-r}\{\underbrace{X_{12}^{(r)} X_{23}^{(s)} X_{31}^{(p+1-r-s)}+X_{13}^{(r)} X_{21}^{(s)} X_{32}^{(p+1-r-s)}}_{=0}\}- \\
& -\sum_{s=1}^{p}(\underbrace{X_{13}^{(s)} X_{31}^{(p+1-s)}}_{=0}+X_{23}^{(s)} X_{32}^{(p+1-s)})-\sum_{r=1}^{p-1} \sum_{s=2}^{p-r} \underbrace{X_{13}^{(r)} X_{22}^{(s)} X_{31}^{(p+1-r-s)}}_{=0} \\
& =-\sum_{s=1}^{p} X_{23}^{(s)} X_{32}^{(p+1-s)} \\
& p_{3 p+i}^{\mathbf{Z}}=-\sum_{s=i}^{p} X_{23}^{(s)} X_{32}^{(p+i-s)}-\sum_{s=i}^{p} \underbrace{X_{13}^{(s)} X_{31}^{(p+i-s)}}_{=0}+ \\
& +\sum_{r=1}^{i-1} \sum_{s=i-r}^{p}\{\underbrace{-X_{13}^{(r)} X_{22}^{(s)} X_{31}^{(p+i-r-s)}+X_{12}^{(r)} X_{23}^{(s)} X_{31}^{(p+i-r-s)}+X_{13}^{(r)} X_{21}^{(s)} X_{32}^{(p+i-r-s)}}_{=0}\}+
\end{aligned}
$$




$$
\begin{aligned}
& +\sum_{r=i}^{p} \sum_{s=1}^{p+i-r-1}\{\underbrace{-X_{13}^{(r)} X_{22}^{(s)} X_{31}^{(p+i-r-s)}+X_{12}^{(r)} X_{23}^{(s)} X_{31}^{(p+i-r-s)}+X_{13}^{(r)} X_{21}^{(s)} X_{32}^{(p+i-r-s)}}_{=0}\} \\
= & -\sum_{s=i}^{p} X_{23}^{(s)} X_{32}^{(p+i-s)}, \quad i=2,3, \ldots, p .
\end{aligned}
$$

Agora, projetando esta variedade sobre as variáveis

$$
X_{i 1}^{(t)}, \quad i=1,2,3 ; \quad t=1,2, \ldots, p
$$

$\mathrm{e}$

$$
X_{1 j}^{(t)}, \quad j=1,2,3 ; t=1,2, \ldots, p
$$

temos, pelas proposições (2.69) e (2.70), que para mostrar a equidimensionalidade de

$$
V\left(\mathbf{Z} \cup\left\{p_{3 j}^{\mathbf{Z}}\right\}_{j=1}^{2 p}\right) \subset k^{9 p}
$$

com dimensão $p$ é equivalente provar que

$$
\widetilde{\mathbb{W}}_{1}:=V\left(\mathbf{A} \cup\left\{q_{3 j}\right\}_{j=1}^{2 p}\right)=V\left(\mathbf{A} \cup\left\{p_{3 j}^{\mathbf{Z}}\right\}_{j=1}^{2 p}\right) \subset k^{9 p-5 p}=k^{4 p}
$$

é equidimensional com dimensão

$$
\operatorname{dim} \widetilde{\mathbb{W}}_{1}=4 p-(p+2 p)=p,
$$

em que

$$
\begin{aligned}
\mathbf{A} & :=\left\{X_{22}^{(i)}\right\}_{i=1}^{p}, \\
q_{31} & :=p_{31}^{\mathbf{Z}}=X_{33}^{(1)}, \\
q_{32} & :=p_{32}^{\mathbf{Z}}=X_{33}^{(2)}-X_{23}^{(1)} X_{32}^{(1)}, \\
q_{33} & :=p_{33}^{\mathbf{Z}}=X_{33}^{(3)}-X_{23}^{(1)} X_{32}^{(2)}-X_{23}^{(2)} X_{32}^{(1)}, \\
q_{3 i} & :=p_{3 i}^{\mathbf{Z}}=X_{33}^{(i)}-\sum_{s=1}^{i-1} X_{23}^{(s)} X_{32}^{(i-s)}, \quad i=4,5, \ldots, p-2, \\
q_{3 p-1} & :=p_{3 p-1}^{\mathbf{Z}}=X_{33}^{(p-1)}-\sum_{s=1}^{p-2} X_{23}^{(s)} X_{32}^{(p-1-s)}, \\
q_{3 p} & :=p_{3 p}^{\mathbf{Z}}=X_{33}^{(p)}-\sum_{s=1}^{p-1} X_{23}^{(s)} X_{32}^{(p-s)}, \\
q_{3 p+1} & :=-p_{3 p+1}^{\mathbf{Z}}=\sum_{s=1}^{p} X_{23}^{(s)} X_{32}^{(p+1-s)}, \\
q_{3 p+i} & :=-p_{3 p+i}^{\mathbf{Z}}=\sum_{s=i}^{p} X_{23}^{(s)} X_{32}^{(p+i-s)}, i=2,3, \ldots, p-3, \\
q_{32 p-2} & :=-p_{32 p-2}^{\mathbf{Z}}=\sum_{s=p-2}^{p} X_{23}^{(s)} X_{32}^{(2 p-2-s)}, \\
q_{32 p-1} & :=-p_{32 p-1}^{\mathbf{Z}}=X_{23}^{(p-1)} X_{32}^{(p)}+X_{23}^{(p)} X_{32}^{(p-1)}, \\
q_{32 p} & :=-p_{32 p}^{\mathbf{Z}}=X_{23}^{(p)} X_{32}^{(p)}
\end{aligned}
$$


e usando a mudança variável $\varphi$

$$
\begin{array}{ll}
X_{22}^{(t)} \longleftrightarrow X_{11}^{(t)}, & t=1,2, \ldots, p \\
X_{23}^{(t)} \longleftrightarrow X_{12}^{(t)}, & t=1,2, \ldots, p \\
X_{32}^{(t)} \longleftrightarrow X_{21}^{(t)}, & t=1,2, \ldots, p \\
X_{33}^{(t)} \longleftrightarrow X_{22}^{(t)}, & t=1,2, \ldots, p
\end{array}
$$

temos $\widetilde{\mathbb{W}}_{1} \cong \varphi\left(\widetilde{\mathbb{W}}_{1}\right) \mathrm{e}$

$$
\varphi\left(\widetilde{\mathbb{W}}_{1}\right)=\varphi\left(V\left(\mathbf{A} \cup\left\{q_{3 j}\right\}_{j=1}^{2 p}\right)\right)=V\left(\varphi(\mathbf{A}) \cup\left\{\varphi\left(q_{3 j}\right)\right\}_{j=1}^{2 p}\right) \subset k^{4 p},
$$

com

$$
\begin{aligned}
\varphi(\mathbf{A}) & =\left\{\varphi\left(X_{22}^{(i)}\right)\right\}_{i=1}^{p}=\left\{X_{11}^{(i)}\right\}_{i=1}^{p}=\left\{p_{1 i}\right\}_{i=1}^{p}, \\
\varphi\left(q_{31}\right) & =\varphi\left(X_{33}^{(1)}\right)=X_{22}^{(1)}=p_{21}, \\
\varphi\left(q_{32}\right) & =\varphi\left(X_{33}^{(2)}-X_{23}^{(1)} X_{32}^{(1)}\right)=X_{22}^{(2)}-X_{12}^{(1)} X_{21}^{(1)}=p_{22}, \\
\varphi\left(q_{33}\right) & =\varphi\left(X_{33}^{(3)}-X_{23}^{(1)} X_{32}^{(2)}-X_{23}^{(2)} X_{32}^{(1)}\right)=X_{22}^{(3)}-X_{12}^{(1)} X_{21}^{(2)}-X_{12}^{(2)} X_{21}^{(1)}=p_{23}, \\
\varphi\left(q_{3 i}\right) & =\varphi\left(X_{33}^{(i)}-\sum_{s=1}^{i-1} X_{23}^{(s)} X_{32}^{(i-s)}\right)=X_{22}^{(i)}-\sum_{s=1}^{i-1} X_{12}^{(s)} X_{21}^{(i-s)}=p_{2 i}, \quad i=4,5, \ldots, p-2, \\
\varphi\left(q_{3 p-1}\right) & =\varphi\left(X_{33}^{(p-1)}-\sum_{s=1}^{p-2} X_{23}^{(s)} X_{32}^{(p-1-s)}\right)=X_{22}^{(p-1)}-\sum_{s=1}^{p-2} X_{12}^{(s)} X_{21}^{(p-1-s)}=p_{2 p-1}, \\
\varphi\left(q_{3 p}\right) & =\varphi\left(X_{33}^{(p)}-\sum_{s=1}^{p-1} X_{23}^{(s)} X_{32}^{(p-s)}\right)=X_{22}^{(p)}-\sum_{s=1}^{p-1} X_{12}^{(s)} X_{21}^{(p-s)}=p_{2 p}, \\
\varphi\left(q_{3 p+1}\right) & =\varphi\left(\sum_{s=1}^{p} X_{23}^{(s)} X_{32}^{(p+1-s)}\right)=\sum_{s=1}^{p} X_{12}^{(s)} X_{21}^{(p+1-s)}=p_{2 p+1}, \\
\varphi\left(q_{3 p+i}\right) & =\varphi\left(\sum_{s=i}^{p} X_{23}^{(s)} X_{32}^{(p+i-s)}\right)=\sum_{s=i}^{p} X_{12}^{(s)} X_{21}^{(p+i-s)}=p_{2 p+i}, \quad i=2,3, \ldots, p-3, \\
\varphi\left(q_{32 p-2}\right) & =\varphi\left(\sum_{s=p-2}^{p} X_{23}^{(s)} X_{32}^{(2 p-2-s)}\right)=\sum_{s=p-2}^{p} X_{12}^{(s)} X_{21}^{(2 p-2-s)}=p_{22 p-2}, \\
\varphi\left(q_{32 p-1}\right) & =\varphi\left(X_{23}^{(p-1)} X_{32}^{(p)}+X_{23}^{(p)} X_{32}^{(p-1)}\right)=X_{12}^{(p-1)} X_{21}^{(p)}+X_{12}^{(p)} X_{21}^{(p-1)}=p_{22 p-1}, \\
\varphi\left(q_{32 p}\right) & =\varphi\left(X_{23}^{(p)} X_{32}^{(p)}\right)=X_{12}^{(p)} X_{21}^{(p)}=p_{22 p} .
\end{aligned}
$$

Portanto, $\widetilde{\mathbb{W}}_{1} \cong \varphi\left(\widetilde{\mathbb{W}}_{1}\right)$ é a variedade de Gelfand-Tsetlin $\mathfrak{G}$ para Yangian $Y_{p}\left(\mathfrak{g l}_{2}\right)$, a qual pelo teorema (5.20) é equidimensional com dimensão

$$
\operatorname{dim} \varphi\left(\widetilde{\mathbb{W}}_{1}\right)=4 p-3 p=p
$$

Corolário 5.22. Para $p>2$, a subvariedade $\mathbb{W}_{1}$ da versão fraca $\mathfrak{G}_{1}$ da variedade de Gelfand-Tsetlin para $Y_{p}\left(\mathfrak{g l}_{3}\right)$ é equidimensional com dimensão $3 p$. 
Demonstração: Pela proposição (5.21) e a notação (5.15)

$$
\mathbb{W}_{1}=\bigcup_{s=1}^{p+1} \mathbb{W}_{1 s}
$$

Proposição 5.23. Para todo $s=2,3, \ldots, p, p+1$ e $p>2$, a subvariedade $\mathbb{W}_{2 s}$ da versão fraca da variedade de Gelfand-Tsetlin para $Y_{p}\left(\mathfrak{g l}_{3}\right)$ é equidimensional com dimensão $3 p$.

Demonstração: Usando a notação (5.15) as subvariedades $\mathbb{W}_{2 s}$ 's são:

$$
\mathbb{W}_{2 s}=V\left(\mathbf{X}_{s} \cup\left\{p_{3 j}^{\mathbf{X}_{s}}\right\}_{j=1}^{2 p}\right) \subset k^{9 p}, \quad s=2,3, \ldots, p, p+1,
$$

em que

$$
\begin{aligned}
\mathbf{X}_{s} & =\left\{X_{11}^{(i)}\right\}_{i=1}^{p} \cup\left\{X_{12}^{(i)}\right\}_{i=1}^{p} \cup\left\{X_{22}^{(i)}\right\}_{i=1}^{p} \cup\left\{X_{21}^{(i)}\right\}_{i=s}^{p} \cup\left\{X_{32}^{(i)}\right\}_{i=p-(s-2)}^{p}, s=2,3, \ldots, p \\
\mathbf{X}_{p+1} & =\left\{X_{11}^{(i)}\right\}_{i=1}^{p} \cup\left\{X_{12}^{(i)}\right\}_{i=1}^{p} \cup\left\{X_{22}^{(i)}\right\}_{i=1}^{p} \cup\left\{X_{32}^{(i)}\right\}_{i=1}^{p} .
\end{aligned}
$$

Mostraremos que $\mathbb{W}_{2 s}$ é equidimensional com

$$
\operatorname{dim}\left(\mathbb{W}_{2 s}\right)=9 p-(\underbrace{4 p}_{=\left|\mathbf{X}_{s}\right|}+2 p)=3 p .
$$

Para isso, pelo corolário (2.67) e a proposição (2.69) é suficiente provar que

$$
V\left(\mathbf{X}_{s} \cup\left\{p_{3 j}^{\mathbf{X}_{s}}\right\}_{j=1,2, \ldots, 2 p} \cup \mathbf{Y}_{s}\right) \subset k^{9 p},
$$

com

$$
\begin{aligned}
\mathbf{Y}_{s} & =\left\{X_{21}^{(i)}\right\}_{i=1}^{s-1} \cup\left\{X_{32}^{(i)}\right\}_{i=1}^{p-(s-2)-1} \cup\left\{X_{23}^{(i)}\right\}_{i=1}^{p}, s=2,3, \ldots, p \\
\mathbf{Y}_{p+1} & =\left\{X_{21}^{(i)}\right\}_{i=1}^{p} \cup\left\{X_{23}^{(i)}\right\}_{i=1}^{p}
\end{aligned}
$$

é equidimensional com dimensão $9 p-(\underbrace{4 p}_{=\left|\mathbf{X}_{s}\right|}+2 p+\underbrace{2 p}_{=\left|\mathbf{Y}_{s}\right|})=p$. Para cada $s=2,3, \ldots, p, p+1$

$$
V\left(\mathbf{X}_{s} \cup\left\{p_{3 j}^{\mathbf{X}_{s}}\right\}_{j=1}^{2 p} \cup \mathbf{Y}_{s}\right)=V\left(\mathbf{Z} \cup\left\{p_{3 j}^{\mathbf{Z}}\right\}_{j=1}^{2 p}\right) \subset k^{9 p},
$$

em que, pela proposição (5.9) e a notação (3.17)

$$
\begin{aligned}
\mathbf{Z} & :=\mathbf{X}_{s} \cup \mathbf{Y}_{s}=\left\{X_{11}^{(i)}\right\}_{i=1}^{p} \cup\left\{X_{12}^{(i)}\right\}_{i=1}^{p} \cup\left\{X_{22}^{(i)}\right\}_{i=1}^{p} \cup\left\{X_{21}^{(i)}\right\}_{i=1}^{p} \cup\left\{X_{23}^{(i)}\right\}_{i=1}^{p} \cup\left\{X_{32}^{(i)}\right\}_{i=1}^{p}, \\
p_{31}^{\mathbf{Z}} & =X_{33}^{(1)}, \\
p_{32}^{\mathbf{Z}} & =X_{33}^{(2)}-\underbrace{X_{23}^{(1)} X_{32}^{(1)}-X_{13}^{(1)} X_{31}^{(1)}}_{=0} \\
& =X_{33}^{(2)}-X_{13}^{(1)} X_{31}^{(1)}, \\
p_{33}^{\mathbf{Z}} & =X_{33}^{(3)}-\underbrace{X_{23}^{(1)} X_{32}^{(2)}-X_{23}^{(2)} X_{32}^{(1)}}_{=0}-\sum_{s=1}^{2} X_{13}^{(s)} X_{31}^{(3-s)}+\underbrace{X_{12}^{(1)} X_{23}^{(1)} X_{31}^{(1)}+X_{13}^{(1)} X_{21}^{(1)} X_{32}^{(1)}}_{=0} \\
& =X_{33}^{(3)}-\sum_{s=1}^{2} X_{13}^{(s)} X_{31}^{(3-s)}, \\
p_{3 i}^{\mathbf{Z}} & =-\sum_{r=1}^{i-2} \sum_{s=2}^{i-r-1} \underbrace{X_{13}^{(r)} X_{22}^{(s)} X_{31}^{(i-r-s)}}_{=0}+X_{33}^{(i)}-\sum_{s=1}^{i-1}(X_{13}^{(s)} X_{31}^{(i-s)}+\underbrace{X_{23}^{(s)} X_{32}^{(i-s)}}_{=0})+
\end{aligned}
$$




$$
\begin{aligned}
& +\sum_{r=1}^{i-2} \sum_{s=1}^{i-r-1}\{\underbrace{X_{12}^{(r)} X_{23}^{(s)} X_{31}^{(i-r-s)}+X_{13}^{(r)} X_{21}^{(s)} X_{32}^{(i-r-s)}}_{=0}\} \\
& =X_{33}^{(i)}-\sum_{s=1}^{i-1} X_{13}^{(s)} X_{31}^{(i-s)}, \quad i=4,5, \ldots, p, \\
& p_{3 p+1}^{\mathbf{Z}}=\sum_{r=1}^{p-1} \sum_{s=1}^{p-r}\{\underbrace{X_{12}^{(r)} X_{23}^{(s)} X_{31}^{(p+1-r-s)}+X_{13}^{(r)} X_{21}^{(s)} X_{32}^{(p+1-r-s)}}_{=0}\}- \\
& -\sum_{s=1}^{p}(X_{13}^{(s)} X_{31}^{(p+1-s)}+\underbrace{X_{23}^{(s)} X_{32}^{(p+1-s)}}_{=0})-\sum_{r=1}^{p-1} \sum_{s=2}^{p-r} \underbrace{X_{13}^{(r)} X_{22}^{(s)} X_{31}^{(p+1-r-s)}}_{=0} \\
& =-\sum_{s=1}^{p} X_{13}^{(s)} X_{31}^{(p+1-s)} \text {, } \\
& p_{3 p+i}^{\mathrm{Z}}=-\sum_{s=i}^{p} \underbrace{X_{23}^{(s)} X_{32}^{(p+i-s)}}_{=0}-\sum_{s=i}^{p} X_{13}^{(s)} X_{31}^{(p+i-s)}+ \\
& +\sum_{r=1}^{i-1} \sum_{s=i-r}^{p}\{\underbrace{-X_{13}^{(r)} X_{22}^{(s)} X_{31}^{(p+i-r-s)}+X_{12}^{(r)} X_{23}^{(s)} X_{31}^{(p+i-r-s)}+X_{13}^{(r)} X_{21}^{(s)} X_{32}^{(p+i-r-s)}}_{=0}\}+ \\
& +\sum_{r=i}^{p} \sum_{s=1}^{p+i-r-1}\{\underbrace{-X_{13}^{(r)} X_{22}^{(s)} X_{31}^{(p+i-r-s)}+X_{12}^{(r)} X_{23}^{(s)} X_{31}^{(p+i-r-s)}+X_{13}^{(r)} X_{21}^{(s)} X_{32}^{(p+i-r-s)}}_{=0}\}, \\
& =-\sum_{s=i}^{p} X_{13}^{(s)} X_{31}^{(p+i-s)}, \quad i=2,3, \ldots, p .
\end{aligned}
$$

Projetando esta variedade sobre as variáveis

$$
X_{i 2}^{(t)}, \quad i=1,2,3 ; \quad t=1,2, \ldots, p
$$

$\mathrm{e}$

$$
X_{2 j}^{(t)}, \quad j=1,2,3 ; \quad t=1,2, \ldots, p
$$

temos, pelas proposições (2.69) e (2.70), que para mostrar a equidimensionalidade de

$$
V\left(\mathbf{Z} \cup\left\{p_{3 j}^{\mathbf{Z}}\right\}_{j=1}^{2 p}\right) \subset k^{9 p}
$$

com dimensão $p$ é equivalente provar que

$$
\widetilde{\mathbb{W}}_{2}:=V\left(\mathbf{A} \cup\left\{q_{3 j}\right\}_{j=1}^{2 p}\right)=V\left(\mathbf{A} \cup\left\{p_{3 j}^{\mathbf{Z}}\right\}_{j=1}^{2 p}\right) \subset k^{9 p-5 p}=k^{4 p}
$$

é equidimensional com dimensão

$$
\operatorname{dim} \widetilde{\mathbb{W}}_{2}=4 p-(p+2 p)=p,
$$

em que

$$
\begin{aligned}
\mathbf{A} & :=\left\{X_{11}^{(i)}\right\}_{i=1}^{p}, \\
q_{31} & :=p_{31}^{\mathbf{Z}}=X_{33}^{(1)}, \\
q_{32} & :=p_{32}^{\mathbf{Z}}=X_{33}^{(2)}-X_{13}^{(1)} X_{31}^{(1)},
\end{aligned}
$$




$$
\begin{aligned}
q_{33} & :=p_{33}^{\mathbf{Z}}=X_{33}^{(3)}-\sum_{s=1}^{2} X_{13}^{(s)} X_{31}^{(3-s)}, \\
q_{3 i} & :=p_{3 i}^{\mathbf{Z}}=X_{33}^{(i)}-\sum_{s=1}^{i-1} X_{13}^{(s)} X_{31}^{(i-s)}, \quad i=4,5, \ldots, p, \\
q_{3 p+1} & :=-p_{3 p+1}^{\mathbf{Z}}=\sum_{s=1}^{p} X_{13}^{(s)} X_{31}^{(p+1-s)}, \\
q_{3 p+i} & :=-p_{3 p+i}^{\mathbf{Z}}=\sum_{s=i}^{p} X_{13}^{(s)} X_{31}^{(p+i-s)}, i=2,3, \ldots, p
\end{aligned}
$$

e usando a mudança de variável $\varphi$

$$
\begin{array}{ll}
X_{11}^{(t)} \longleftrightarrow X_{11}^{(t)}, & t=1,2, \ldots, p \\
X_{13}^{(t)} \longleftrightarrow X_{12}^{(t)}, & t=1,2, \ldots, p \\
X_{31}^{(t)} \longleftrightarrow X_{21}^{(t)}, & t=1,2, \ldots, p \\
X_{33}^{(t)} \longleftrightarrow X_{22}^{(t)}, & t=1,2, \ldots, p,
\end{array}
$$

temos $\widetilde{\mathbb{W}}_{2} \cong \varphi\left(\widetilde{\mathbb{W}}_{2}\right) \mathrm{e}$

$$
\varphi\left(\widetilde{\mathbb{W}}_{2}\right)=\varphi\left(V\left(\mathbf{A} \cup\left\{q_{3 j}\right\}_{j=1}^{2 p}\right)\right)=V\left(\varphi(\mathbf{A}) \cup\left\{\varphi\left(q_{3 j}\right)\right\}_{j=1}^{2 p}\right) \subset k^{4 p},
$$

com

$$
\begin{aligned}
\varphi(\mathbf{A}) & =\left\{X_{11}^{(i)}\right\}_{i=1}^{p}=\left\{p_{1 i}\right\}_{i=1}^{p}, \\
\varphi\left(q_{31}\right) & =\varphi\left(X_{33}^{(1)}\right)=X_{22}^{(1)}=p_{21}, \\
\varphi\left(q_{32}\right) & =\varphi\left(X_{33}^{(2)}-X_{13}^{(1)} X_{31}^{(1)}\right)=X_{22}^{(2)}-X_{12}^{(1)} X_{21}^{(1)}=p_{22}, \\
\varphi\left(q_{33}\right) & =\varphi\left(X_{33}^{(3)}-\sum_{s=1}^{2} X_{13}^{(s)} X_{31}^{(3-s)}\right)=X_{22}^{(3)}-\sum_{s=1}^{2} X_{12}^{(s)} X_{21}^{(3-s)}=p_{23}, \\
\varphi\left(q_{3 i}\right) & =\varphi\left(X_{33}^{(i)}-\sum_{s=1}^{i-1} X_{13}^{(s)} X_{31}^{(i-s)}\right)=X_{22}^{(i)}-\sum_{s=1}^{i-1} X_{12}^{(s)} X_{21}^{(i-s)}=p_{2 i}, \quad i=4,5, \ldots, p, \\
\varphi\left(q_{3 p+1}\right) & =\varphi\left(\sum_{s=1}^{p} X_{13}^{(s)} X_{31}^{(p+1-s)}\right)=\sum_{s=1}^{p} X_{12}^{(s)} X_{21}^{(p+1-s)}=p_{2 p+1}, \\
\varphi\left(q_{3 p+i}\right) & =\varphi\left(\sum_{s=i}^{p} X_{13}^{(s)} X_{31}^{(p+i-s)}\right)=\sum_{s=i}^{p} X_{12}^{(s)} X_{21}^{(p+i-s)}=p_{2 p+i}, \quad i=2,3, \ldots, p .
\end{aligned}
$$

Portanto, $\widetilde{\mathbb{W}}_{2} \cong \varphi\left(\widetilde{\mathbb{W}}_{2}\right)$ é a variedade de Gelfand-Tsetlin $\mathfrak{G}$ para Yangian $Y_{p}\left(\mathfrak{g l}_{2}\right)$, a qual pelo teorema (5.20) é equidimensional com dimensão

$$
\operatorname{dim} \varphi\left(\widetilde{\mathbb{W}}_{2}\right)=4 p-3 p=p
$$

Corolário 5.24. Para $p>2$, a subvariedade $\mathbb{W}_{2}$ da versão fraca $\mathfrak{G}_{1}$ da variedade de Gelfand-Tsetlin para $Y_{p}\left(\mathfrak{g l}_{3}\right)$ é equidimensional com dimensão $3 p$. 
Demonstração: Pela proposição (5.23) e a notação (5.15)

$$
\mathbb{W}_{2}=\bigcup_{s=2}^{p+1} \mathbb{W}_{2 s}
$$

Agora estamos em condições de provar nosso resultado principal.

Teorema 5.25. A variedade $\mathfrak{G}_{1}$ para $Y_{p}\left(\mathfrak{g l}_{3}\right)$ é equidimensional com dimensão

$$
\operatorname{dim} \mathfrak{G}_{1}=3 p .
$$

Demonstração: Provemos por indução sobre $p$.

Pela proposição (5.11), temos que $\mathfrak{G}_{1}$ para $Y_{1}\left(\mathfrak{g l}_{3}\right)$ é equidimensional com dimensão

$$
\operatorname{dim} \mathfrak{G}_{1}=3 .
$$

Similarmente, da proposição (5.12), temos que $\mathfrak{G}_{1}$ para $Y_{2}\left(\mathfrak{g l}_{3}\right)$ é equidimensional com dimensão

$$
\operatorname{dim} \mathfrak{G}_{1}=6 .
$$

Agora suponha $p>2$ e que a variedade $\mathfrak{G}_{1}$ para $Y_{p-1}\left(\mathfrak{g l}_{3}\right)$ é equidimensional com dimensão $3(p-1)$.

Como consequência do corolário (5.19), temos a decomposição

$$
\mathfrak{G}_{1}=\mathbb{W}^{p} \cup \mathbb{W}_{1} \cup \mathbb{W}_{2} \text {, para } p \geq 3 \text {. }
$$

Pelos corolários (5.22) e (5.24), as componentes $\mathbb{W}_{1} \mathrm{e} \mathbb{W}_{2}$ são equidimensionais com dimensão $3 p$.

Mostremos que $\mathbb{W}^{p}$ é equidimensional com dimensão $3 p$. Usando a notação (5.15), temos

$$
\mathbb{W}^{p}=V\left(\mathbf{X} \cup\left\{p_{3 j}\right\}_{j=1}^{3 p-3}\right) \subset k^{9 p},
$$

com

$$
\mathbf{X}=\left\{X_{11}^{(i)}\right\}_{i=1}^{p} \cup\left\{X_{12}^{(i)}\right\}_{i=1}^{p} \cup\left\{X_{22}^{(i)}\right\}_{i=1}^{p} \cup\left\{X_{32}^{(p)}, X_{21}^{(p)}, X_{13}^{(p)}\right\} .
$$

Para provar que $\mathbb{W}^{p}$ é equidimensional com $\operatorname{dim}\left(\mathbb{W}^{p}\right)=3 p$, pelo corolário (2.67) e a proposição (2.69), é suficiente mostrar que

$$
V\left(\mathbf{X} \cup\left\{p_{3 j}\right\}_{j=1,2}^{3 p-3} \cup\left\{X_{23}^{(p)}, X_{31}^{(p)}, X_{33}^{(p)}\right\}\right) \subset k^{9 p}
$$

é equidimensional com dimensão $9 p-(6 p+3)=3 p-3=3(p-1)$. Claramente

$$
V\left(\mathbf{Y} \cup\left\{p_{3 j}^{\mathbf{Y}}\right\}_{j=1}^{3 p-3}\right)=V\left(\mathbf{X} \cup\left\{p_{3 j}\right\}_{j=1}^{3 p-3} \cup\left\{X_{23}^{(p)}, X_{31}^{(p)}, X_{33}^{(p)}\right\}\right) \subset k^{9 p},
$$

em que, como consequência da proposição (5.9) e a notação (3.17)

$$
\begin{aligned}
\mathbf{Y} & =\left\{X_{11}^{(i)}\right\}_{i=1}^{p-1} \cup\left\{X_{12}^{(i)}\right\}_{i=1}^{p-1} \cup\left\{X_{22}^{(i)}\right\}_{i=1}^{p-1} \cup\left\{X_{i j}^{(p)}\right\}_{i, j=1}^{3}, \\
p_{31}^{\mathbf{Y}} & =X_{33}^{(1)}, \\
p_{32}^{\mathbf{Y}} & =X_{33}^{(2)}-X_{23}^{(1)} X_{32}^{(1)}-X_{13}^{(1)} X_{31}^{(1)}, \\
p_{33}^{\mathbf{Y}} & =X_{33}^{(3)}-X_{23}^{(1)} X_{32}^{(2)}-X_{23}^{(2)} X_{32}^{(1)}-\sum_{s=1}^{2} X_{13}^{(s)} X_{31}^{(3-s)}+\underbrace{X_{12}^{(1)} X_{23}^{(1)} X_{31}^{(1)}}_{=0}+X_{13}^{(1)} X_{21}^{(1)} X_{32}^{(1)} \\
& =X_{33}^{(3)}-X_{23}^{(1)} X_{32}^{(2)}-X_{23}^{(2)} X_{32}^{(1)}-\sum_{s=1}^{2} X_{13}^{(s)} X_{31}^{(3-s)}+X_{13}^{(1)} X_{21}^{(1)} X_{32}^{(1)},
\end{aligned}
$$


para cada $i=4,5, \ldots, p-1$ temos

$$
\begin{aligned}
p_{3 i}^{\mathbf{Y}}= & -\sum_{r=1}^{i-2} \sum_{s=2}^{i-r-1} \underbrace{X_{13}^{(r)} X_{22}^{(s)} X_{31}^{(i-r-s)}}_{=0}+X_{33}^{(i)}-\sum_{s=1}^{i-1}\left(X_{13}^{(s)} X_{31}^{(i-s)}+X_{23}^{(s)} X_{32}^{(i-s)}\right)+ \\
& +\sum_{r=1}^{i-2} \sum_{s=1}^{i-r-1}\{\underbrace{X_{12}^{(r)} X_{23}^{(s)} X_{31}^{(i-r-s)}}_{=0}+X_{13}^{(r)} X_{21}^{(s)} X_{32}^{(i-r-s)}\} \\
= & X_{33}^{(i)}-\sum_{s=1}^{i-1}\left(X_{13}^{(s)} X_{31}^{(i-s)}+X_{23}^{(s)} X_{32}^{(i-s)}\right)+\sum_{r=1}^{i-2} \sum_{s=1}^{i-r-1} X_{13}^{(r)} X_{21}^{(s)} X_{32}^{(i-r-s)},
\end{aligned}
$$

análogamente, temos

$$
\begin{aligned}
p_{3 p}^{\mathbf{Y}}= & -\sum_{r=1}^{p-2} \sum_{s=2}^{p-r-1} \underbrace{X_{13}^{(r)} X_{22}^{(s)} X_{31}^{(p-r-s)}}_{=0}+\underbrace{X_{33}^{(p)}}_{=0}-\sum_{s=1}^{p-1}\left(X_{13}^{(s)} X_{31}^{(p-s)}+X_{23}^{(s)} X_{32}^{(p-s)}\right)+ \\
& +\sum_{r=1}^{p-2} \sum_{s=1}^{p-r-1}\{\underbrace{X_{12}^{(r)} X_{23}^{(s)} X_{31}^{(p-r-s)}}_{=0}+X_{13}^{(r)} X_{21}^{(s)} X_{32}^{(p-r-s)}\} \\
= & -\sum_{s=1}^{p-1}\left(X_{13}^{(s)} X_{31}^{(p-s)}+X_{23}^{(s)} X_{32}^{(p-s)}\right)+\sum_{r=1}^{p-2} \sum_{s=1}^{p-r-1} X_{13}^{(r)} X_{21}^{(s)} X_{32}^{(p-r-s)}, \\
p_{3 p+1}^{\mathbf{Y}}= & \sum_{r=1}^{p-1} \sum_{s=1}^{p-r}\{\underbrace{X_{12}^{(r)} X_{23}^{(s)} X_{31}^{(p+1-r-s)}}_{=0}+X_{13}^{(r)} X_{21}^{(s)} X_{32}^{(p+1-r-s)}\}- \\
& -\sum_{s=1}^{p}\left(X_{13}^{(s)} X_{31}^{(p+1-s)}+X_{23}^{(s)} X_{32}^{(p+1-s)}\right)-\sum_{r=1}^{p-1} \sum_{s=2}^{p-r} \underbrace{(r)}_{=0} X_{22}^{(s)} X_{31}^{(p+1-r-s)} \\
= & \sum_{r=1}^{p-1} \sum_{s=1}^{p-r} X_{13}^{(r)} X_{21}^{(s)} X_{32}^{(p+1-r-s)}-\sum_{s=1}^{p}\left(X_{13}^{(s)} X_{31}^{(p+1-s)}+X_{23}^{(s)} X_{32}^{(p+1-s)}\right) \\
= & \sum_{r=1}^{p-1} \sum_{s=1}^{p-r} X_{13}^{(r)} X_{21}^{(s)} X_{32}^{(p+1-r-s)}-\sum_{s=2}^{p-1}\left(X_{13}^{(s)} X_{31}^{(p+1-s)}+X_{23}^{(s)} X_{32}^{(p+1-s)}\right)- \\
& -(\underbrace{X_{13}^{(1)} X_{31}^{(p)}+X_{23}^{(1)} X_{32}^{(p)}+X_{13}^{(p)} X_{31}^{(1)}+X_{23}^{(p)} X_{32}^{(1)}}_{=0}) \\
= & \sum_{r=1}^{p-1} \sum_{s=1}^{p-r} X_{13}^{(r)} X_{21}^{(s)} X_{32}^{(p+1-r-s)}-\sum_{s=2}^{p-1}\left(X_{13}^{(s)} X_{31}^{(p+1-s)}+X_{23}^{(s)} X_{32}^{(p+1-s)}\right), \\
& (p-2
\end{aligned}
$$

para cada $i=2,3, \ldots, p-2$ temos

$$
\begin{aligned}
p_{3 p+i}^{\mathbf{Y}}= & -\sum_{s=i}^{p} X_{23}^{(s)} X_{32}^{(p+i-s)}-\sum_{s=i}^{p} X_{13}^{(s)} X_{31}^{(p+i-s)}+ \\
& +\sum_{r=1}^{i-1} \sum_{s=i-r}^{p}\left\{-X_{13}^{(r)} X_{22}^{(s)} X_{31}^{(p+i-r-s)}+X_{12}^{(r)} X_{23}^{(s)} X_{31}^{(p+i-r-s)}+X_{13}^{(r)} X_{21}^{(s)} X_{32}^{(p+i-r-s)}\right\}+ \\
& +\sum_{r=i}^{p} \sum_{s=1}^{p+i-r-1}\left\{-X_{13}^{(r)} X_{22}^{(s)} X_{31}^{(p+i-r-s)}+X_{12}^{(r)} X_{23}^{(s)} X_{31}^{(p+i-r-s)}+X_{13}^{(r)} X_{21}^{(s)} X_{32}^{(p+i-r-s)}\right\} \\
= & -\sum_{s=i+1}^{p-1} X_{23}^{(s)} X_{32}^{(p+i-s)} \underbrace{-X_{23}^{(i)} X_{32}^{(p)}-X_{23}^{(p)} X_{32}^{(i)}}_{=0}-\sum_{s=i+1}^{p-1} X_{13}^{(s)} X_{31}^{(p+i-s)} \underbrace{-X_{13}^{(i)} X_{31}^{(p)}-X_{13}^{(p)} X_{31}^{(i)}}_{=0}+
\end{aligned}
$$




$$
\begin{aligned}
& +\sum_{r=1}^{i-1} \sum_{s=i-r+1}^{p-1}\{\underbrace{-X_{13}^{(r)} X_{22}^{(s)} X_{31}^{(p+i-r-s)}+X_{12}^{(r)} X_{23}^{(s)} X_{31}^{(p+i-r-s)}}_{=0}+X_{13}^{(r)} X_{21}^{(s)} X_{32}^{(p+i-r-s)}\}+ \\
& +\sum_{r=1}^{i-1}\{\underbrace{-X_{13}^{(r)} X_{22}^{(i-r)} X_{31}^{(p)}+X_{12}^{(r)} X_{23}^{(i-r)} X_{31}^{(p)}+X_{13}^{(r)} X_{21}^{(i-r)} X_{32}^{(p)}}_{=0}\}+ \\
& +\sum_{r=1}^{i-1}\{\underbrace{-X_{13}^{(r)} X_{22}^{(p)} X_{31}^{(i-r)}+X_{12}^{(r)} X_{23}^{(p)} X_{31}^{(i-r)}+X_{13}^{(r)} X_{21}^{(p)} X_{32}^{(i-r)}}_{=0}\}+ \\
& +\sum_{r=i}^{p-1} \sum_{s=1}^{p+i-r-1}\{\underbrace{-X_{13}^{(r)} X_{22}^{(s)} X_{31}^{(p+i-r-s)}+X_{12}^{(r)} X_{23}^{(s)} X_{31}^{(p+i-r-s)}}_{=0}+X_{13}^{(r)} X_{21}^{(s)} X_{32}^{(p+i-r-s)}\}+ \\
& +\sum_{s=1}^{i-1}\{\underbrace{-X_{13}^{(p)} X_{22}^{(s)} X_{31}^{(i-s)}+X_{12}^{(p)} X_{23}^{(s)} X_{31}^{(i-s)}+X_{13}^{(p)} X_{21}^{(s)} X_{32}^{(i-s)}}_{=0}\} \text {, } \\
& =-\sum_{s=i+1}^{p-1} X_{23}^{(s)} X_{32}^{(p+i-s)}-\sum_{s=i+1}^{p-1} X_{13}^{(s)} X_{31}^{(p+i-s)}+ \\
& +\sum_{r=1}^{i-1} \sum_{s=i-r+1}^{p-1} X_{13}^{(r)} X_{21}^{(s)} X_{32}^{(p+i-r-s)}+\sum_{r=i}^{p-1} \sum_{s=1}^{p+i-r-1} X_{13}^{(r)} X_{21}^{(s)} X_{32}^{(p+i-r-s)}
\end{aligned}
$$

continuando com o mesmo raciocinio

$$
\begin{aligned}
& p_{32 p-1}^{\mathbf{Y}}=\underbrace{-\sum_{s=p-1}^{p} X_{23}^{(s)} X_{32}^{(2 p-1-s)}-\sum_{s=p-1}^{p} X_{13}^{(s)} X_{31}^{(2 p-1-s)}}_{=0}+ \\
& +\sum_{r=1}^{p-2} \sum_{s=p-1-r}^{p}\{\underbrace{-X_{13}^{(r)} X_{22}^{(s)} X_{31}^{(2 p-1-r-s)}+X_{12}^{(r)} X_{23}^{(s)} X_{31}^{(2 p-1-r-s)}}_{=0}+X_{13}^{(r)} X_{21}^{(s)} X_{32}^{(2 p-1-r-s)}\}+ \\
& +\sum_{r=p-1}^{p} \sum_{s=1}^{2 p-r-2}\{\underbrace{-X_{13}^{(r)} X_{22}^{(s)} X_{31}^{(2 p-1-r-s)}+X_{12}^{(r)} X_{23}^{(s)} X_{31}^{(2 p-1-r-s)}}_{=0}+X_{13}^{(r)} X_{21}^{(s)} X_{32}^{(2 p-1-r-s)}\} \\
& =\sum_{r=1}^{p-2} \sum_{s=p-1-r}^{p} X_{13}^{(r)} X_{21}^{(s)} X_{32}^{(2 p-1-r-s)}+\sum_{r=p-1}^{p} \sum_{s=1}^{2 p-r-2} X_{13}^{(r)} X_{21}^{(s)} X_{32}^{(2 p-1-r-s)} \\
& =\sum_{r=1}^{p-2} \sum_{s=p-r}^{p-1} X_{13}^{(r)} X_{21}^{(s)} X_{32}^{(2 p-1-r-s)}+\underbrace{\sum_{r=1}^{p-2} X_{13}^{(r)} X_{21}^{(p-1-r)} X_{32}^{(p)}+\sum_{r=1}^{p-2} X_{13}^{(r)} X_{21}^{(p)} X_{32}^{(p-1-r)}}_{=0}+ \\
& +\sum_{s=1}^{p-1} X_{13}^{(p-1)} X_{21}^{(s)} X_{32}^{(p-s)}+\sum_{s=1}^{p-2} \underbrace{X_{13}^{(p)} X_{21}^{(s)} X_{32}^{(p-1-s)}}_{=0} \\
& =\sum_{r=1}^{p-2} \sum_{s=p-r}^{p-1} X_{13}^{(r)} X_{21}^{(s)} X_{32}^{(2 p-1-r-s)}+\sum_{s=1}^{p-1} X_{13}^{(p-1)} X_{21}^{(s)} X_{32}^{(p-s)}, \\
& p_{32 p}^{\mathbf{Y}}=\underbrace{-X_{23}^{(p)} X_{32}^{(p)}-X_{13}^{(p)} X_{31}^{(p)}}_{=0}+ \\
& +\sum_{r=1}^{p-1} \sum_{s=p-r}^{p}\{\underbrace{-X_{13}^{(r)} X_{22}^{(s)} X_{31}^{(2 p-r-s)}+X_{12}^{(r)} X_{23}^{(s)} X_{31}^{(2 p-r-s)}}_{=0}+X_{13}^{(r)} X_{21}^{(s)} X_{32}^{(2 p-r-s)}\}+
\end{aligned}
$$




$$
\begin{aligned}
& +\sum_{s=1}^{p-1}\{\underbrace{-X_{13}^{(p)} X_{22}^{(s)} X_{31}^{(p-s)}+X_{12}^{(p)} X_{23}^{(s)} X_{31}^{(p-s)}+X_{13}^{(p)} X_{21}^{(s)} X_{32}^{(p-s)}}_{=0}\} \\
= & \sum_{r=1}^{p-1} \sum_{s=p-r}^{p} X_{13}^{(r)} X_{21}^{(s)} X_{32}^{(2 p-r-s)} \\
= & \sum_{r=1}^{p-1} \sum_{s=p-r+1}^{p-1} X_{13}^{(r)} X_{21}^{(s)} X_{32}^{(2 p-r-s)}+\underbrace{\sum_{r=1}^{p-1} X_{13}^{(r)} X_{21}^{(p-r)} X_{32}^{(p)}+\sum_{r=1}^{p-1} X_{13}^{(r)} X_{21}^{(p)} X_{32}^{(p-r)}}_{=0} \\
= & \sum_{r=1}^{p-1} \sum_{s=p-r+1}^{p-1} X_{13}^{(r)} X_{21}^{(s)} X_{32}^{(2 p-r-s)},
\end{aligned}
$$

para cada $i=1,2, \ldots, p-4$ temos

$$
\begin{aligned}
p_{32 p+i}^{\mathbf{Y}} & =\sum_{r=i}^{p} \sum_{s=p+i-r}^{p}\{\underbrace{-X_{13}^{(r)} X_{22}^{(s)} X_{31}^{(2 p+i-r-s)}+X_{12}^{(r)} X_{23}^{(s)} X_{31}^{(2 p+i-r-s)}}_{=0}+X_{13}^{(r)} X_{21}^{(s)} X_{32}^{(2 p+i-r-s)}\} \\
& =\sum_{r=i}^{p} \sum_{s=p+i-r}^{p} X_{13}^{(r)} X_{21}^{(s)} X_{32}^{(2 p+i-r-s)} \\
& =\sum_{r=i+1}^{p-1} \sum_{s=p+i-r}^{p} X_{13}^{(r)} X_{21}^{(s)} X_{32}^{(2 p+i-r-s)}+\underbrace{X_{13}^{(i)} X_{21}^{(p)} X_{32}^{(p)}+\sum_{s=i}^{p} X_{13}^{(p)} X_{21}^{(s)} X_{32}^{(p+i-s)}}_{=0} \\
& =\sum_{r=i+1}^{p-1} \sum_{s=p+i-r}^{p} X_{13}^{(r)} X_{21}^{(s)} X_{32}^{(2 p+i-r-s)} \underbrace{p-1}_{=0} \sum_{r=i+1}^{p-1} X_{13}^{(r)} X_{21}^{(s)} X_{32}^{(2 p+i-r-s)}+p+i-r+1 \\
& =\sum_{r=i+1}^{\sum^{p-1} X_{13}^{(r)} X_{21}^{(p+i-r)} X_{32}^{(p)}+\sum_{r=i+1}^{p-1} X_{13}^{(r)} X_{21}^{(p)} X_{32}^{(p+i-r)}} \\
& =\sum_{r=i+1}^{p-1} \sum_{s=p+i-r+1}^{p-1} X_{13}^{(r)} X_{21}^{(s)} X_{32}^{(2 p+i-r-s)},
\end{aligned}
$$

análogamente tem-se

$$
\begin{aligned}
p_{33 p-3}^{\mathbf{Y}}= & \sum_{r=p-3}^{p} \sum_{s=2 p-3-r}^{p}\{\underbrace{-X_{13}^{(r)} X_{22}^{(s)} X_{31}^{(3 p-3-r-s)}+X_{12}^{(r)} X_{23}^{(s)} X_{31}^{(3 p-3-r-s)}}_{=0}+X_{13}^{(r)} X_{21}^{(s)} X_{32}^{(3 p-3-r-s)}\} \\
= & \sum_{r=p-3}^{p} \sum_{s=2 p-3-r}^{p} X_{13}^{(r)} X_{21}^{(s)} X_{32}^{(3 p-3-r-s)} \\
= & \underbrace{X_{13}^{(p-3)} X_{21}^{(p)} X_{32}^{(p)}+\sum_{s=p-1}^{p} X_{13}^{(p-2)} X_{21}^{(s)} X_{32}^{(2 p-1-s)}}_{=0}+\sum_{s=p-2}^{p} X_{13}^{(p-1)} X_{21}^{(s)} X_{32}^{(2 p-2-s)} \\
& +\underbrace{\sum_{s=p-3}^{p} X_{13}^{(p)} X_{21}^{(s)} X_{32}^{(2 p-3-s)}}_{=0} \\
= & \sum_{s=p-2}^{p} X_{13}^{(p-1)} X_{21}^{(s)} X_{32}^{(2 p-2-s)}
\end{aligned}
$$




$$
\begin{aligned}
& =\underbrace{X_{13}^{(p-1)} X_{21}^{(p-2)} X_{32}^{(p)}}_{=0}+X_{13}^{(p-1)} X_{21}^{(p-1)} X_{32}^{(p-1)}+\underbrace{X_{13}^{(p-1)} X_{21}^{(p)} X_{32}^{(p-2)}}_{=0} \\
& =X_{13}^{(p-1)} X_{21}^{(p-1)} X_{32}^{(p-1)} .
\end{aligned}
$$

Agora, projetando a variedade sobre as variáveis

$$
X_{i j}^{(p)}, \quad i=1,2,3,
$$

temos, pela proposição (2.70), que para mostrar a equidimensionalidade de

$$
V\left(\mathbf{Y} \cup\left\{p_{3 j}^{\mathbf{Y}}\right\}_{j=1}^{3 p-3}\right) \subset k^{9 p}
$$

com dimensão 3(p-1), é equivalente provar que

$$
\widetilde{\mathbb{W}}^{p}=V\left(\mathbf{Z} \cup\left\{q_{3 j}^{\mathbf{Z}}\right\}_{j=1}^{3 p-3}\right) \subset k^{9 p-9},
$$

com

$$
\mathbf{Z}=\left\{X_{11}^{(i)}\right\}_{i=1}^{p-1} \cup\left\{X_{12}^{(i)}\right\}_{i=1}^{p-1} \cup\left\{X_{22}^{(i)}\right\}_{i=1}^{p-1}
$$

$\mathrm{e}$

$$
q_{3 j}^{\mathbf{Z}}=p_{3 j}^{\mathbf{Y}}
$$

é equidimensional com $\operatorname{dim}\left(\widetilde{\mathbb{W}}^{p}\right)=9 p-9-6(p-1)=3(p-1)$. Mas, notemos que $\widetilde{\mathbb{W}}^{p}$ é a versão fraca $\mathfrak{G}_{1}$ para $Y_{p-1}\left(\mathfrak{g l}_{3}\right)$. 


\section{Apêndice A}

\section{Contas}

Neste apêndice apresentaremos alguns resultados necessários para o capítulo 5 .

Todas as operações do tipo soma e produto neste apêndice são sobre um anel comutativo.

Proposição A.1. Seja $p \in \mathbb{Z}_{>0}$ :

1. Para todo $3 \leq i \leq p$

$$
\sum_{\substack{r+s+t=i \\ r, s, t \neq 0}} x_{s} y_{s} z_{t}=\sum_{r=1}^{i-2} \sum_{s=1}^{i-r-1} x_{r} y_{s} z_{i-r-s}
$$

2.

$$
\sum_{\substack{r+s+t=p+1 \\ 1 \leq r, s, t \leq p}} x_{s} y_{s} z_{t}=\sum_{r=1}^{p-1} \sum_{s=1}^{p-r} x_{r} y_{s} z_{p+1-r-s}
$$

3. Para todo $2 \leq i \leq p$

$$
\sum_{\substack{r+s+t=p+i \\ 1 \leq r, s, t \leq p}} x_{s} y_{s} z_{t}=\sum_{r=1}^{i-1} \sum_{s=i-r}^{p} x_{r} y_{s} z_{p+i-r-s}+\sum_{r=i}^{p} \sum_{s=1}^{p+i-r-1} x_{r} y_{s} z_{p+i-r-s} .
$$

4. Para todo $1 \leq i \leq p$

$$
\sum_{\substack{r+s+t=2 p+i \\ 1 \leq r, s, t \leq p}} x_{s} y_{s} z_{t}=\sum_{r=i}^{p} \sum_{s=p+i-r}^{p} x_{r} y_{s} z_{2 p+i-r-s} .
$$

Demonstração:

1.

$$
\begin{aligned}
\sum_{\substack{r+s+t=i \\
r, s, t \neq 0}} x_{s} y_{s} z_{t} & =x_{1} \sum_{\substack{s+t=i-1 \\
s, t \neq 0}} y_{s} z_{t}+x_{2} \sum_{\substack{s+t=i-2 \\
s, t \neq 0}} y_{s} z_{t}+x_{3} \sum_{\substack{s+t=i-3 \\
s, t \neq 0}} y_{s} z_{t}+\cdots+x_{i-2} \sum_{\substack{s+t=2 \\
s, t \neq 0}} y_{s} z_{t} \\
& =x_{1} \sum_{s=1}^{i-2} y_{s} z_{i-1-s}+x_{2} \sum_{s=1}^{i-3} y_{s} z_{i-2-s}+x_{3} \sum_{s=1}^{i-4} y_{s} z_{i-3-s}+\cdots+x_{i-2} \sum_{s=1} y_{s} z_{2-s} \\
& =\sum_{r=1}^{i-2} x_{r} \sum_{s=1}^{i-r-1} y_{s} z_{i-r-s} .
\end{aligned}
$$


2.

$$
\begin{aligned}
\sum_{\substack{r+s+t=p+1 \\
1 \leq r, s, t \leq p}} x_{s} y_{s} z_{t} & =x_{1} \sum_{\substack{s+t=p \\
1 \leq s, t \leq p-1}} y_{s} z_{t}+x_{2} \sum_{\substack{s+t=p-1 \\
1 \leq s, t \leq p-2}} y_{s} z_{t}+x_{3} \sum_{\substack{s+t=p-2 \\
1 \leq s, t \leq p-3}} y_{s} z_{t}+\cdots+x_{p-1} \sum_{\substack{s+t=2 \\
1 \leq s, t \leq 1}} y_{s} z_{t} \\
& =x_{1} \sum_{s=1}^{p-1} y_{s} z_{p-s}+x_{2} \sum_{s=1}^{p-2} y_{s} z_{p-1-s}+x_{3} \sum_{s=1}^{p-3} y_{s} z_{p-2-s}+\cdots+x_{p-1} \sum_{s=1}^{1} y_{s} z_{2-s} \\
& =\sum_{r=1}^{p-1} x_{r} \sum_{s=1}^{p-r} y_{s} z_{p+1-r-s} .
\end{aligned}
$$

3. Para $2 \leq i \leq p$

$$
\begin{aligned}
\sum_{\substack{r+s+t=p+i \\
1 \leq r, s, t \leq p}} x_{s} y_{s} z_{t}= & x_{1} \sum_{\substack{s+t=p+i-1 \\
i-1 \leq s, t \leq p}} y_{s} z_{t}+x_{2} \sum_{\substack{s+t=p+i-2 \\
i-2 \leq s, t \leq p}} y_{s} z_{t}+\cdots+x_{i-1} \sum_{\substack{s+t=p+1 \\
1 \leq s, t \leq p}} y_{s} z_{t}+ \\
& +x_{i} \sum_{\substack{s+t=p \\
1 \leq s, t \leq p-1}} y_{s} z_{t}+x_{i+1} \sum_{\substack{s+t=p-1 \\
1 \leq s, t \leq p-2}} y_{s} z_{t}+\cdots+x_{p} \sum_{\substack{s+t=i \\
1 \leq s, t \leq i-1}} y_{s} z_{t} \\
= & x_{1} \sum_{s=i-1}^{p} y_{s} z_{p+i-1-s}+x_{2} \sum_{s=i-2}^{p} y_{s} z_{p+i-2-s}+\cdots+x_{i-1} \sum_{s=1}^{p} y_{s} z_{p+1-s}+ \\
& +x_{i} \sum_{s=1}^{p-1} y_{s} z_{p-s}+x_{i+1} \sum_{s=1}^{p-2} y_{s} z_{p-1-s}+\cdots+x_{p} \sum_{s=1}^{i-1} y_{s} z_{i-s} \\
= & \sum_{r=1}^{i-1} x_{r} \sum_{s=i-r}^{p} y_{s} z_{p+i-r-s}+\sum_{r=i}^{p} x_{r} \sum_{s=1}^{p+i-r-1} y_{s} z_{p+i-r-s .} .
\end{aligned}
$$

4. Para $1 \leq i \leq p$

$$
\begin{aligned}
\sum_{\substack{r+s+t=2 p+i \\
1 \leq r, s, t \leq p}} x_{s} y_{s} z_{t}= & x_{i} y_{p} z_{p}+x_{i+1} \sum_{\substack{s+t=2 p-1 \\
p-1 \leq s, t \leq p}} y_{s} z_{t}+x_{i+2} \sum_{\substack{s+t=2 p-2 \\
s-2 \leq s, t \leq p}} y_{s} z_{t}+\cdots+ \\
& +x_{p-2} \sum_{\substack{s+t=p+i+2 \\
i+2 \leq s, t \leq p}} y_{s} z_{t}+x_{p-1} \sum_{\substack{s+t=p+i+1 \\
i+1 \leq s, t \leq p}} y_{s} z_{t}+x_{p} \sum_{\substack{s+t=p+i \\
i \leq s, t \leq p}} y_{s} z_{t} \\
= & x_{i} y_{p} z_{p}+x_{i+1} \sum_{s=p-1}^{p} y_{s} z_{2 p-1-s}+x_{i+2} \sum_{s=p-2}^{p} y_{s} z_{2 p-2-s}+\cdots+ \\
& +x_{p-2} \sum_{s=i+2}^{p} y_{s} z_{p+i+2-s}+x_{p-1} \sum_{s=i+1}^{p} y_{s} z_{p+i+1-s}+x_{p} \sum_{s=i}^{p} y_{s} z_{p+i-s} \\
= & \sum_{r=i}^{p} x_{r} \sum_{s=p+i-r}^{p} y_{s} z_{2 p+i-r-s} .
\end{aligned}
$$


Proposição A.2. Para quaisquer $i, p \in \mathbb{Z}_{>0}, 1 \leq i \leq p$

$$
\begin{aligned}
\sum_{r+s+t=1} x_{s} y_{s} z_{t} & =x_{0} y_{0} z_{1}+x_{0} y_{1} z_{0}+x_{1} y_{0} z_{0}, \\
\sum_{r+s+t=2} x_{s} y_{s} z_{t} & =x_{0} y_{0} z_{2}+x_{0} y_{2} z_{0}+x_{2} y_{0} z_{0}+x_{0} y_{1} z_{1}+y_{0} x_{1} z_{1}+z_{0} x_{1} y_{1}, \\
\sum_{r+s+t=i} x_{s} y_{s} z_{t}= & x_{0} y_{0} z_{i}+x_{0} y_{i} z_{0}+x_{i} y_{0} z_{0}+x_{0} \sum_{s=1}^{i-1} y_{s} z_{i-s}+y_{0} \sum_{s=1}^{i-1} x_{s} z_{i-s}+ \\
& +z_{0} \sum_{s=1}^{i-1} x_{s} y_{i-s}+\sum_{r=1}^{i-2} \sum_{s=1}^{i-r-1} x_{r} y_{s} z_{i-r-s}, \quad i=3,4,5, \ldots, p .
\end{aligned}
$$

Demonstração: Para $i \geq 3$

$$
\begin{aligned}
& \sum_{r+s+t=i} x_{s} y_{s} z_{t}=x_{0} \sum_{s+t=i} y_{s} z_{t}+x_{1} \sum_{s+t=i-1} y_{s} z_{t}+x_{2} \sum_{s+t=i-2} y_{s} z_{t}+\cdots+x_{i-2} \sum_{s+t=2} y_{s} z_{t}+ \\
& +x_{i-1} \sum_{s+t=1} y_{s} z_{t}+x_{i} \sum_{s+t=0} y_{s} z_{t} \\
& =x_{0} \sum_{s=0}^{i} y_{s} z_{i-s}+x_{1} \sum_{s=0}^{i-1} y_{s} z_{i-1-s}+x_{2} \sum_{s=0}^{i-2} y_{s} z_{i-2-s}+\cdots+ \\
& +x_{i-2} \sum_{s=0}^{2} y_{s} z_{2-s}+x_{i-1} y_{0} z_{1}+x_{i-1} y_{1} z_{0}+x_{i} y_{0} z_{0} \\
& =x_{0} \sum_{s=0}^{i} y_{s} z_{i-s}+\sum_{r=1}^{i-2} x_{r} \sum_{s=0}^{i-r} y_{s} z_{i-r-s}+x_{i-1} y_{0} z_{1}+x_{i-1} y_{1} z_{0}+x_{i} y_{0} z_{0} \\
& =x_{0} y_{0} z_{i}+x_{0} y_{i} z_{0}+x_{0} \sum_{s=1}^{i-1} y_{s} z_{i-s}+x_{i-1} y_{0} z_{1}+x_{i-1} y_{1} z_{0}+x_{i} y_{0} z_{0}+ \\
& +\sum_{r=1}^{i-2}\left(x_{r} y_{0} z_{i-r}+x_{r} y_{i-r} z_{0}+x_{r} \sum_{s=1}^{i-r-1} y_{s} z_{i-r-s}\right) \\
& =x_{0} y_{0} z_{i}+x_{0} y_{i} z_{0}+x_{0} \sum_{s=1}^{i-1} y_{s} z_{i-s}+x_{i-1} y_{0} z_{1}+x_{i-1} y_{1} z_{0}+x_{i} y_{0} z_{0}+ \\
& +\sum_{r=1}^{i-2} x_{r} y_{0} z_{i-r}+\sum_{r=1}^{i-2} x_{r} y_{i-r} z_{0}+\sum_{r=1}^{i-2} x_{r} \sum_{s=1}^{i-r-1} y_{s} z_{i-r-s} \\
& =x_{0} y_{0} z_{i}+x_{0} y_{i} z_{0}+x_{i} y_{0} z_{0}+x_{0} \sum_{s=1}^{i-1} y_{s} z_{i-s}+x_{i-1} y_{0} z_{1}+\sum_{r=1}^{i-2} x_{r} y_{0} z_{i-r}+ \\
& +x_{i-1} y_{1} z_{0}+\sum_{r=1}^{i-2} x_{r} y_{i-r} z_{0}+\sum_{r=1}^{i-2} x_{r} \sum_{s=1}^{i-r-1} y_{s} z_{i-r-s} \\
& =x_{0} y_{0} z_{i}+x_{0} y_{i} z_{0}+x_{i} y_{0} z_{0}+x_{0} \sum_{s=1}^{i-1} y_{s} z_{i-s}+\sum_{r=1}^{i-1} x_{r} y_{0} z_{i-r}+ \\
& +\sum_{r=1}^{i-1} x_{r} y_{i-r} z_{0}+\sum_{r=1}^{i-2} x_{r} \sum_{s=1}^{i-r-1} y_{s} z_{i-r-s}
\end{aligned}
$$


Proposição A.3. Para quaisquer $i, p \in \mathbb{Z}_{>0}, 1 \leq i \leq p$

$$
\begin{aligned}
\sum_{\substack{r+s+t=p+1 \\
0 \leq r, s, t \leq p}} x_{s} y_{s} z_{t}= & x_{0} \sum_{s=1}^{p} y_{s} z_{p+1-s}+y_{0} \sum_{s=1}^{p} x_{s} z_{p+1-s}+z_{0} \sum_{s=1}^{p} x_{s} y_{p+1-s}+\sum_{r=1}^{p-1} \sum_{s=1}^{p-r} x_{r} y_{s} z_{p+1-r-s}, \\
\sum_{\substack{r+s+t=p+i \\
0 \leq r, s, t \leq p}} x_{s} y_{s} z_{t}= & x_{0} \sum_{s=i}^{p} y_{s} z_{p+i-s}+y_{0} \sum_{s=i}^{p} x_{s} z_{p+i-s}+z_{0} \sum_{s=i}^{p} x_{s} y_{p+i-s}+ \\
& +\sum_{r=1}^{i-1} \sum_{s=i-r}^{p} x_{r} y_{s} z_{p+i-r-s}+\sum_{r=i}^{p} \sum_{s=1}^{p+i-r-1} x_{r} y_{s} z_{p+i-r-s}, \quad i \geq 2 .
\end{aligned}
$$

Demonstração:

$$
\begin{aligned}
\sum_{\substack{r+s+t=p+i \\
0 \leq r, s, t \leq p}} x_{s} y_{s} z_{t}= & x_{0} \sum_{\substack{s+t=p+i \\
0 \leq s, t \leq p}} y_{s} z_{t}+x_{1} \sum_{\substack{s+t=p+i-1 \\
0 \leq s, t \leq p}} y_{s} z_{t}+\cdots+x_{i-1} \sum_{\substack{s+t=p+1 \\
0 \leq s, t \leq p}} y_{s} z_{t}+ \\
& +x_{i} \sum_{s+t=p} y_{s} z_{t}+x_{i+1} \sum_{s+t=p-1} y_{s} z_{t}+x_{i+2} \sum_{s+t=p-2} y_{s} z_{t}+ \\
& +\cdots+x_{p-2} \sum_{s+t=i+2} y_{s} z_{t}+x_{p-1} \sum_{s+t=i+1} y_{s} z_{t}+x_{p} \sum_{s+t=i} y_{s} z_{t} \\
= & x_{0} \sum_{s=i}^{p} y_{s} z_{p+i-s}+x_{1} \sum_{s=i-1}^{p} y_{s} z_{p+i-1-s}+\cdots+x_{i-1} \sum_{s=1}^{p} y_{s} z_{p+1-s}+ \\
& +x_{i} \sum_{s=0}^{p} y_{s} z_{p-s}+x_{i+1} \sum_{s=0}^{p-1} y_{s} z_{p-1-s}+x_{i+2} \sum_{s=0}^{p-2} y_{s} z_{p-2-s}+ \\
& +\cdots+x_{p-2} \sum_{s=0}^{i+2} y_{s} z_{i+2-s}+x_{p-1} \sum_{s=0}^{i+1} y_{s} z_{i+1-s}+x_{p} \sum_{s=0}^{i} y_{s} z_{i-s}
\end{aligned}
$$

Agora, se $i=1$,

$$
\begin{aligned}
\sum_{\substack{r+s+t=p+1 \\
0 \leq r, s, t \leq p}} x_{s} y_{s} z_{t}= & x_{0} \sum_{s=1}^{p} y_{s} z_{p+1-s}+x_{1} \sum_{s=0}^{p} y_{s} z_{p-s}+x_{2} \sum_{s=0}^{p-1} y_{s} z_{p-1-s}+\cdots+ \\
& +x_{p-2} \sum_{s=0}^{3} y_{s} z_{3-s}+x_{p-1} \sum_{s=0}^{2} y_{s} z_{2-s}+x_{p} \sum_{s=0}^{1} y_{s} z_{1-s} \\
= & x_{0} \sum_{s=1}^{p} y_{s} z_{p+1-s}+\sum_{r=1}^{p-1} x_{r} \sum_{s=0}^{p+1-r} y_{s} z_{p+1-r-s}+x_{p} y_{0} z_{1}+x_{p} y_{1} z_{0} \\
= & x_{p} y_{0} z_{1}+x_{p} y_{1} z_{0}+x_{0} \sum_{s=1}^{p} y_{s} z_{p+1-s}+\sum_{r=1}^{p-1} x_{r}\left(y_{0} z_{p+1-r}+\sum_{s=1}^{p-r} y_{s} z_{p+1-r-s}+y_{p+1-r} z_{0}\right) \\
= & x_{p} y_{0} z_{1}+x_{p} y_{1} z_{0}+x_{0} \sum_{s=1}^{p} y_{s} z_{p+1-s}+\sum_{r=1}^{p-1} x_{r} y_{0} z_{p+1-r}+ \\
& +\sum_{r=1}^{p-1} x_{r} y_{p+1-r} z_{0}+\sum_{r=1}^{p-1} x_{r} \sum_{s=1}^{p-r} y_{s} z_{p+1-r-s} \\
= & x_{0} \sum_{s=1}^{p} y_{s} z_{p+1-s}+\sum_{r=1}^{p} x_{r} y_{0} z_{p+1-r}+\sum_{r=1}^{p} x_{r} y_{p+1-r} z_{0}+\sum_{r=1}^{p-1} x_{r} \sum_{s=1}^{p-r} y_{s} z_{p+1-r-s}
\end{aligned}
$$


e se $2 \leq i \leq p$

$$
\begin{aligned}
\sum_{\substack{r+s+t=p+i \\
0 \leq r, s, t \leq p}} x_{s} y_{s} z_{t}= & x_{0} \sum_{s=i}^{p} y_{s} z_{p+i-s}+\sum_{r=1}^{i-1} x_{r} \sum_{s=i-r}^{p} y_{s} z_{p+i-r-s}+x_{i} \sum_{s=0}^{p} y_{s} z_{p-s}+\sum_{r=i+1}^{p} x_{r} \sum_{s=0}^{p+i-r} y_{s} z_{p+i-r-s} \\
= & x_{0} \sum_{s=i}^{p} y_{s} z_{p+i-s}+x_{i} \sum_{s=0}^{p} y_{s} z_{p-s}+\sum_{r=1}^{i-1} x_{r} \sum_{s=i-r}^{p} y_{s} z_{p+i-r-s}+ \\
& +\sum_{r=i+1}^{p} x_{r}\left(y_{0} z_{p+i-r}+\sum_{s=1}^{p+i-r-1} y_{s} z_{p+i-r-s}+y_{p+i-r} z_{0}\right) \\
= & x_{0} \sum_{s=i}^{p} y_{s} z_{p+i-s}+x_{i} y_{0} z_{p}+x_{i} \sum_{s=1}^{p-1} y_{s} z_{p-s}+x_{i} y_{p} z_{0}+ \\
& +\sum_{r=1}^{i-1} x_{r} \sum_{s=i-r}^{p} y_{s} z_{p+i-r-s}+\sum_{r=i+1}^{p} x_{r} y_{0} z_{p+i-r}+ \\
& +\sum_{r=i+1}^{p} x_{r} \sum_{s=1}^{p+i-r-1} y_{s} z_{p+i-r-s}+\sum_{r=i+1}^{p} x_{r} y_{p+i-r} z_{0} \\
= & x_{0} \sum_{s=i}^{p} y_{s} z_{p+i-s}+x_{i} y_{0} z_{p}+\sum_{r=i+1}^{p} x_{r} y_{0} z_{p+i-r}+x_{i} y_{p} z_{0}+ \\
& +\sum_{r=i+1}^{p} x_{r} y_{p+i-r} z_{0}+\sum_{r=1}^{i-1} x_{r} \sum_{s=i-r}^{p} y_{s} z_{p+i-r-s}+x_{i} \sum_{s=1}^{p-1} y_{s} z_{p-s}+ \\
& +\sum_{r=i+1}^{p} x_{r} \sum_{s=1}^{p+i-r-1} y_{s} z_{p+i-r-s} \\
= & x_{0} \sum_{s=i}^{p} y_{s} z_{p+i-s}+y_{0} \sum_{r=i}^{p} x_{r} z_{p+i-r}+z_{0} \sum_{r=i}^{p} x_{r} y_{p+i-r}+ \\
& +\sum_{r=1}^{i-1} x_{r} \sum_{s=i-r}^{p} y_{s} z_{p+i-r-s}+\sum_{r=i}^{p} x_{r} \sum_{s=1}^{p+i-r-1} y_{s} z_{p+i-r-s} . \\
&
\end{aligned}
$$

Proposição A.4. Para quaisquer $i, p \in \mathbb{Z}_{>0}, 1 \leq i \leq p$

$$
\sum_{\substack{r+s+t=2 p+i \\ 0 \leq r, s, t \leq p}} x_{s} y_{s} z_{t}=\sum_{r=i}^{p} \sum_{s=p+i-r}^{p} x_{r} y_{s} z_{2 p+i-r-s} .
$$

\section{Demonstração:}

$$
\begin{aligned}
\sum_{\substack{r+s+t=2 p+i \\
0 \leq r, s, t \leq p}} x_{s} y_{s} z_{t}= & x_{i} y_{p} z_{p}+x_{i+1} \sum_{\substack{s+t=2 p-1 \\
0 \leq s, t \leq p}} y_{s} z_{t}+x_{i+2} \sum_{\substack{s+t=2 p-2 \\
0 \leq s, t \leq p}} y_{s} z_{t}+\cdots+ \\
& +x_{p-2} \sum_{\substack{s+t=p+i+2 \\
0 \leq s, t \leq p}} y_{s} z_{t}+x_{p-1} \sum_{\substack{s+t=p+i+1 \\
0 \leq s, t \leq p}} y_{s} z_{t}+x_{p} \sum_{\substack{s+t=p+i \\
0 \leq s, t \leq p}} y_{s} z_{t}
\end{aligned}
$$




$$
\begin{aligned}
= & x_{i} y_{p} z_{p}+x_{i+1} \sum_{s=p-1}^{p} y_{s} z_{2 p-1-s}+x_{i+2} \sum_{s=p-2}^{p} y_{s} z_{2 p-2-s}+\cdots+ \\
& +x_{p-2} \sum_{s=i+2}^{p} y_{s} z_{p+i+2-s}+x_{p-1} \sum_{s=i+1}^{p} y_{s} z_{p+i+1-s}+x_{p} \sum_{s=i}^{p} y_{s} z_{p+i-s} \\
= & \sum_{r=i}^{p} x_{r} \sum_{s=p+i-r}^{p} y_{s} z_{2 p+i-r-s} .
\end{aligned}
$$




\section{Apêndice B}

\section{Decomposição da variedade de Gelfand-Tsetlin $\mathfrak{G}$ para $Y_{2}\left(\mathfrak{g l}_{3}\right)$}

Neste apêndice apresentaremos todos os detalhes da decomposição em componentes irredutíveis, da variedade de Gelfand-Tsetlin $\mathfrak{G}$ para Yangian $Y_{2}\left(\mathfrak{g l}_{3}\right)$, tal decomposição foi usada na prova da proposição (5.12).

\section{B.1 $\mathfrak{G}=W_{1} \cup W_{2}$}

Como consequência da proposição (5.9), pode concluirse que a variedade de Gelfand-Tsetlin $\mathfrak{G}$ para $Y_{2}\left(\mathfrak{g l}_{3}\right)$ é

$$
\mathfrak{G}=V\left(\left\{p_{i j}\right\}_{\substack{i=1,2,3 \\ j=1,2, \ldots, 2 i}}\right) \subset k^{18}
$$

com

$$
\begin{aligned}
p_{11}= & X_{11}^{(1)} \\
p_{12}= & X_{11}^{(2)}, \\
p_{21}= & X_{22}^{(1)}, \\
p_{22}= & X_{22}^{(2)}-X_{12}^{(1)} X_{21}^{(1)}, \\
p_{23}= & X_{12}^{(1)} X_{21}^{(2)}+X_{12}^{(2)} X_{21}^{(1)}, \\
p_{24}= & X_{12}^{(2)} X_{21}^{(2)} \\
p_{31}= & X_{33}^{(1)}, \\
p_{32}= & X_{33}^{(2)}-X_{23}^{(1)} X_{32}^{(1)}-X_{13}^{(1)} X_{31}^{(1)}, \\
p_{33}= & X_{12}^{(1)} X_{23}^{(1)} X_{31}^{(1)}+X_{13}^{(1)} X_{21}^{(1)} X_{32}^{(1)}-X_{13}^{(1)} X_{31}^{(2)}-X_{23}^{(1)} X_{32}^{(2)}-X_{13}^{(2)} X_{31}^{(1)}-X_{23}^{(2)} X_{32}^{(1)}, \\
p_{34}= & -X_{23}^{(2)} X_{32}^{(2)}-X_{13}^{(2)} X_{31}^{(2)}-X_{13}^{(1)} \underbrace{X_{22}^{(1)}}_{=0} X_{31}^{(2)}+X_{12}^{(1)} X_{23}^{(1)} X_{31}^{(2)}+X_{13}^{(1)} X_{21}^{(1)} X_{32}^{(2)}-X_{13}^{(1)} X_{22}^{(2)} X_{31}^{(1)}+ \\
& +X_{12}^{(1)} X_{23}^{(2)} X_{31}^{(1)}+X_{13}^{(1)} X_{21}^{(2)} X_{32}^{(1)}-X_{13}^{(2)} \underbrace{X_{22}^{(1)}}_{=0} X_{31}^{(1)}+X_{12}^{(2)} X_{23}^{(1)} X_{31}^{(1)}+X_{13}^{(2)} X_{21}^{(1)} X_{32}^{(1)} \\
= & -X_{23}^{(2)} X_{32}^{(2)}-X_{13}^{(2)} X_{31}^{(2)}+X_{12}^{(1)} X_{23}^{(1)} X_{31}^{(2)}+X_{13}^{(1)} X_{21}^{(1)} X_{32}^{(2)}-X_{13}^{(1)} X_{22}^{(2)} X_{31}^{(1)}+ \\
& +X_{12}^{(1)} X_{23}^{(2)} X_{31}^{(1)}+X_{13}^{(1)} X_{21}^{(2)} X_{32}^{(1)}+X_{12}^{(2)} X_{23}^{(1)} X_{31}^{(1)}+X_{13}^{(2)} X_{21}^{(1)} X_{32}^{(1)}, \\
p_{35}= & -X_{13}^{(1)} X_{22}^{(2)} X_{31}^{(2)}+X_{12}^{(1)} X_{23}^{(2)} X_{31}^{(2)}+X_{13}^{(1)} X_{21}^{(2)} X_{32}^{(2)}-X_{13}^{(2)} \underbrace{X_{22}^{(1)}}_{=0} X_{31}^{(2)}+X_{12}^{(2)} X_{23}^{(1)} X_{31}^{(2)}+ \\
& +X_{13}^{(2)} X_{21}^{(1)} X_{32}^{(2)}-X_{13}^{(2)} X_{22}^{(2)} X_{31}^{(1)}+X_{12}^{(2)} X_{23}^{(2)} X_{31}^{(1)}+X_{13}^{(2)} X_{21}^{(2)} X_{32}^{(1)}
\end{aligned}
$$




$$
\begin{aligned}
= & -X_{13}^{(1)} X_{22}^{(2)} X_{31}^{(2)}+X_{12}^{(1)} X_{23}^{(2)} X_{31}^{(2)}+X_{13}^{(1)} X_{21}^{(2)} X_{32}^{(2)}+X_{12}^{(2)} X_{23}^{(1)} X_{31}^{(2)}+X_{13}^{(2)} X_{21}^{(1)} X_{32}^{(2)}- \\
& -X_{13}^{(2)} X_{22}^{(2)} X_{31}^{(1)}+X_{12}^{(2)} X_{23}^{(2)} X_{31}^{(1)}+X_{13}^{(2)} X_{21}^{(2)} X_{32}^{(1)} \\
p_{36}= & -X_{13}^{(2)} X_{22}^{(2)} X_{31}^{(2)}+X_{12}^{(2)} X_{23}^{(2)} X_{31}^{(2)}+X_{13}^{(2)} X_{21}^{(2)} X_{32}^{(2)} .
\end{aligned}
$$

Usando a notação (3.17), tem-se $V=W_{1} \cup W_{2}$ com

$$
\begin{aligned}
W_{1} & =V\left(X_{11}^{(1)}, X_{11}^{(2)}, X_{22}^{(1)}, p_{22}^{\mathbf{X}}, p_{23}^{\mathbf{X}}, X_{12}^{(2)}, X_{33}^{(1)}, p_{32}^{\mathbf{X}}, p_{33}^{\mathbf{X}}, p_{34}^{\mathbf{X}}, p_{35}^{\mathbf{X}}, p_{36}^{\mathbf{X}}\right) \subset k^{18} \\
\mathbf{X} & =\left\{X_{11}^{(1)}, X_{11}^{(2)}, X_{22}^{(1)}, X_{12}^{(2)}, X_{33}^{(1)}\right\}
\end{aligned}
$$

$\mathrm{e}$

$$
\begin{aligned}
W_{2} & =V\left(X_{11}^{(1)}, X_{11}^{(2)}, X_{22}^{(1)}, p_{22}^{\mathbf{X}}, p_{23}^{\mathbf{X}}, X_{21}^{(2)}, X_{33}^{(1)}, p_{32}^{\mathbf{X}}, p_{33}^{\mathbf{X}}, p_{34}^{\mathbf{X}}, p_{35}^{\mathbf{X}}, p_{36}^{\mathbf{X}}\right) \subset k^{18} \\
\mathbf{X} & =\left\{X_{11}^{(1)}, X_{11}^{(2)}, X_{22}^{(1)}, X_{21}^{(2)}, X_{33}^{(1)}\right\} .
\end{aligned}
$$

B.2 $W_{1}=W_{11} \cup W_{12} \cup W_{13} \cup W_{14}$

Para

$$
W_{1}=V\left(X_{11}^{(1)}, X_{11}^{(2)}, X_{22}^{(1)}, p_{22}^{\mathbf{x}}, p_{23}^{\mathbf{X}}, X_{12}^{(2)}, X_{33}^{(1)}, p_{32}^{\mathbf{x}}, p_{33}^{\mathbf{x}}, p_{34}^{\mathbf{x}}, p_{35}^{\mathbf{X}}, p_{36}^{\mathbf{X}}\right) \subset k^{18},
$$

temos

$$
\begin{aligned}
& \mathbf{X}=\left\{X_{11}^{(1)}, X_{11}^{(2)}, X_{22}^{(1)}, X_{12}^{(2)}, X_{33}^{(1)}\right\}, \\
& p_{22}^{\mathbf{X}}=X_{22}^{(2)}-X_{12}^{(1)} X_{21}^{(1)} \\
& p_{23}^{\mathbf{X}}=X_{12}^{(1)} X_{21}^{(2)}+\underbrace{X_{12}^{(2)} X_{21}^{(1)}}_{=0} \\
& =X_{12}^{(1)} X_{21}^{(2)} \text {, } \\
& p_{32}^{\mathbf{X}}=X_{33}^{(2)}-X_{23}^{(1)} X_{32}^{(1)}-X_{13}^{(1)} X_{31}^{(1)} \text {, } \\
& p_{33}^{\mathbf{X}}=X_{12}^{(1)} X_{23}^{(1)} X_{31}^{(1)}+X_{13}^{(1)} X_{21}^{(1)} X_{32}^{(1)}-X_{13}^{(1)} X_{31}^{(2)}-X_{23}^{(1)} X_{32}^{(2)}-X_{13}^{(2)} X_{31}^{(1)}-X_{23}^{(2)} X_{32}^{(1)} \text {, } \\
& p_{34}^{\mathbf{X}}=-X_{23}^{(2)} X_{32}^{(2)}-X_{13}^{(2)} X_{31}^{(2)}+X_{12}^{(1)} X_{23}^{(1)} X_{31}^{(2)}+X_{13}^{(1)} X_{21}^{(1)} X_{32}^{(2)}-X_{13}^{(1)} X_{22}^{(2)} X_{31}^{(1)}+ \\
& +X_{12}^{(1)} X_{23}^{(2)} X_{31}^{(1)}+X_{13}^{(1)} X_{21}^{(2)} X_{32}^{(1)}+\underbrace{X_{12}^{(2)} X_{23}^{(1)} X_{31}^{(1)}}_{=0}+X_{13}^{(2)} X_{21}^{(1)} X_{32}^{(1)} \\
& =-X_{23}^{(2)} X_{32}^{(2)}-X_{13}^{(2)} X_{31}^{(2)}+X_{12}^{(1)} X_{23}^{(1)} X_{31}^{(2)}+X_{13}^{(1)} X_{21}^{(1)} X_{32}^{(2)}-X_{13}^{(1)} X_{22}^{(2)} X_{31}^{(1)}+ \\
& +X_{12}^{(1)} X_{23}^{(2)} X_{31}^{(1)}+X_{13}^{(1)} X_{21}^{(2)} X_{32}^{(1)}+X_{13}^{(2)} X_{21}^{(1)} X_{32}^{(1)} \text {, } \\
& p_{35}^{\mathbf{x}}=-X_{13}^{(1)} X_{22}^{(2)} X_{31}^{(2)}+X_{12}^{(1)} X_{23}^{(2)} X_{31}^{(2)}+X_{13}^{(1)} X_{21}^{(2)} X_{32}^{(2)}+\underbrace{X_{12}^{(2)} X_{23}^{(1)} X_{31}^{(2)}}_{=0}+X_{13}^{(2)} X_{21}^{(1)} X_{32}^{(2)}- \\
& -X_{13}^{(2)} X_{22}^{(2)} X_{31}^{(1)}+\underbrace{X_{12}^{(2)} X_{23}^{(2)} X_{31}^{(1)}}_{=0}+X_{13}^{(2)} X_{21}^{(2)} X_{32}^{(1)} \\
& =-X_{13}^{(1)} X_{22}^{(2)} X_{31}^{(2)}+X_{12}^{(1)} X_{23}^{(2)} X_{31}^{(2)}+X_{13}^{(1)} X_{21}^{(2)} X_{32}^{(2)}+X_{13}^{(2)} X_{21}^{(1)} X_{32}^{(2)}-X_{13}^{(2)} X_{22}^{(2)} X_{31}^{(1)}+X_{13}^{(2)} X_{21}^{(2)} X_{32}^{(1)} \text {, } \\
& p_{36}^{\mathbf{X}}=-X_{13}^{(2)} X_{22}^{(2)} X_{31}^{(2)}+\underbrace{X_{12}^{(2)} X_{23}^{(2)} X_{31}^{(2)}}_{=0}+X_{13}^{(2)} X_{21}^{(2)} X_{32}^{(2)} \\
& =-X_{13}^{(2)} X_{22}^{(2)} X_{31}^{(2)}+X_{13}^{(2)} X_{21}^{(2)} X_{32}^{(2)} \\
& =X_{13}^{(2)}\left(X_{21}^{(2)} X_{32}^{(2)}-X_{22}^{(2)} X_{31}^{(2)}\right) \text {. }
\end{aligned}
$$


Logo $W_{1}=W_{11} \cup W_{12} \cup W_{13} \cup W_{14}$, com

$$
\begin{aligned}
& W_{11}=V\left(X_{11}^{(1)}, X_{11}^{(2)}, X_{22}^{(1)}, p_{22}^{\mathbf{X}}, X_{12}^{(1)}, X_{12}^{(2)}, X_{33}^{(1)}, p_{32}^{\mathbf{X}}, p_{33}^{\mathbf{X}}, p_{34}^{\mathbf{X}}, p_{35}^{\mathbf{X}}, X_{13}^{(2)}\right) \subset k^{18} \\
& \mathbf{X}=\left\{X_{11}^{(1)}, X_{11}^{(2)}, X_{22}^{(1)}, X_{33}^{(1)}, X_{12}^{(2)}, X_{12}^{(1)}, X_{13}^{(2)}\right\} \text {, } \\
& W_{12}=V\left(X_{11}^{(1)}, X_{11}^{(2)}, X_{22}^{(1)}, p_{22}^{\mathbf{x}}, X_{12}^{(1)}, X_{12}^{(2)}, X_{33}^{(1)}, p_{32}^{\mathbf{x}}, p_{33}^{\mathbf{x}}, p_{34}^{\mathbf{x}}, p_{35}^{\mathbf{x}}, \frac{p_{36}^{\mathbf{X}}}{X_{13}^{(2)}}\right) \subset k^{18} \\
& \mathbf{X}=\left\{X_{11}^{(1)}, X_{11}^{(2)}, X_{22}^{(1)}, X_{33}^{(1)}, X_{12}^{(2)}, X_{12}^{(1)}\right\} \text {, } \\
& W_{13}=V\left(X_{11}^{(1)}, X_{11}^{(2)}, X_{22}^{(1)}, p_{22}^{\mathbf{X}}, X_{21}^{(2)}, X_{12}^{(2)}, X_{33}^{(1)}, p_{32}^{\mathbf{X}}, p_{33}^{\mathbf{X}}, p_{34}^{\mathbf{X}}, p_{35}^{\mathbf{X}}, X_{13}^{(2)}\right) \subset k^{18} \\
& \mathbf{X}=\left\{X_{11}^{(1)}, X_{11}^{(2)}, X_{22}^{(1)}, X_{33}^{(1)}, X_{12}^{(2)}, X_{21}^{(2)}, X_{13}^{(2)}\right\}, \\
& \text { e } \\
& W_{14}=V\left(X_{11}^{(1)}, X_{11}^{(2)}, X_{22}^{(1)}, p_{22}^{\mathbf{x}}, X_{21}^{(2)}, X_{12}^{(2)}, X_{33}^{(1)}, p_{32}^{\mathbf{x}}, p_{33}^{\mathbf{x}}, p_{34}^{\mathbf{x}}, p_{35}^{\mathbf{x}}, \frac{p_{36}^{\mathbf{X}}}{X_{13}^{(2)}}\right) \subset k^{18} \\
& \mathbf{X}=\left\{X_{11}^{(1)}, X_{11}^{(2)}, X_{22}^{(1)}, X_{33}^{(1)}, X_{12}^{(2)}, X_{21}^{(2)}\right\} .
\end{aligned}
$$

Vale a pena ressaltar que pelo quociente $\frac{p_{36}^{\mathrm{X}}}{X_{13}^{(2)}}$, queremos dizer

$$
\frac{p_{36}^{\mathbf{X}}}{X_{13}^{(2)}}=X_{21}^{(2)} X_{32}^{(2)}-X_{22}^{(2)} X_{31}^{(2)} \text {. }
$$

\section{B.2.1 Decomposição de $W_{11}$}

Fazendo o mesmo raciocinio das variedades anteriores deste apêndice, temos que para

$$
W_{11}=V\left(X_{11}^{(1)}, X_{11}^{(2)}, X_{22}^{(1)}, p_{22}^{\mathbf{X}}, X_{12}^{(1)}, X_{12}^{(2)}, X_{33}^{(1)}, p_{32}^{\mathbf{x}}, p_{33}^{\mathbf{X}}, p_{34}^{\mathbf{x}}, p_{35}^{\mathbf{X}}, X_{13}^{(2)}\right) \subset k^{18},
$$

com

$$
\begin{aligned}
& \mathbf{X}=\left\{X_{11}^{(1)}, X_{11}^{(2)}, X_{22}^{(1)}, X_{12}^{(1)}, X_{12}^{(2)}, X_{33}^{(1)}, X_{13}^{(2)}\right\}, \\
& p_{22}^{\mathbf{X}}=X_{22}^{(2)}-\underbrace{X_{12}^{(1)} X_{21}^{(1)}}_{=0} \\
& =X_{22}^{(2)} \text {, } \\
& p_{32}^{\mathbf{X}}=X_{33}^{(2)}-X_{23}^{(1)} X_{32}^{(1)}-X_{13}^{(1)} X_{31}^{(1)} \text {, } \\
& p_{33}^{\mathbf{X}}=\underbrace{X_{12}^{(1)} X_{23}^{(1)} X_{31}^{(1)}}_{=0}+X_{13}^{(1)} X_{21}^{(1)} X_{32}^{(1)}-X_{13}^{(1)} X_{31}^{(2)}-X_{23}^{(1)} X_{32}^{(2)}-\underbrace{X_{13}^{(2)} X_{31}^{(1)}}_{=0}-X_{23}^{(2)} X_{32}^{(1)} \\
& =X_{13}^{(1)} X_{21}^{(1)} X_{32}^{(1)}-X_{13}^{(1)} X_{31}^{(2)}-X_{23}^{(1)} X_{32}^{(2)}-X_{23}^{(2)} X_{32}^{(1)} \text {, } \\
& p_{34}^{\mathbf{X}}=-X_{23}^{(2)} X_{32}^{(2)}-\underbrace{X_{13}^{(2)} X_{31}^{(2)}+X_{12}^{(1)} X_{23}^{(1)} X_{31}^{(2)}}_{=0}+X_{13}^{(1)} X_{21}^{(1)} X_{32}^{(2)}-\underbrace{X_{13}^{(1)} X_{22}^{(2)} X_{31}^{(1)}}_{=0}+ \\
& +\underbrace{X_{12}^{(1)} X_{23}^{(2)} X_{31}^{(1)}}_{=0}+X_{13}^{(1)} X_{21}^{(2)} X_{32}^{(1)}+\underbrace{X_{13}^{(2)} X_{21}^{(1)} X_{32}^{(1)}}_{=0} \\
& =-X_{23}^{(2)} X_{32}^{(2)}+X_{13}^{(1)} X_{21}^{(1)} X_{32}^{(2)}+X_{13}^{(1)} X_{21}^{(2)} X_{32}^{(1)} \text {, }
\end{aligned}
$$




$$
\begin{aligned}
p_{35}^{\mathbf{X}} & =\underbrace{-X_{13}^{(1)} X_{22}^{(2)} X_{31}^{(2)}+X_{12}^{(1)} X_{23}^{(2)} X_{31}^{(2)}}_{=0}+X_{13}^{(1)} X_{21}^{(2)} X_{32}^{(2)}+\underbrace{X_{13}^{(2)} X_{21}^{(1)} X_{32}^{(2)}-X_{13}^{(2)} X_{22}^{(2)} X_{31}^{(1)}+X_{13}^{(2)} X_{21}^{(2)} X_{32}^{(1)}}_{=0} \\
& =X_{13}^{(1)} X_{21}^{(2)} X_{32}^{(2)} .
\end{aligned}
$$

Novamente, $W_{11}=W_{111} \cup W_{112} \cup W_{113}$, onde

$$
\begin{aligned}
W_{111} & =V\left(X_{11}^{(1)}, X_{11}^{(2)}, X_{22}^{(1)}, X_{22}^{(2)}, X_{12}^{(1)}, X_{12}^{(2)}, X_{33}^{(1)}, p_{32}^{\mathbf{X}}, p_{33}^{\mathbf{X}}, p_{34}^{\mathbf{x}}, X_{13}^{(1)}, X_{13}^{(2)}\right) \subset k^{18} \\
\mathbf{X} & =\left\{X_{11}^{(1)}, X_{11}^{(2)}, X_{22}^{(1)}, X_{22}^{(2)}, X_{12}^{(1)}, X_{12}^{(2)}, X_{33}^{(1)}, X_{13}^{(1)}, X_{13}^{(2)}\right\}, \\
W_{112} & =V\left(X_{11}^{(1)}, X_{11}^{(2)}, X_{22}^{(1)}, X_{22}^{(2)}, X_{12}^{(1)}, X_{12}^{(2)}, X_{33}^{(1)}, p_{32}^{\mathbf{x}}, p_{33}^{\mathbf{x}}, p_{34}^{\mathbf{X}}, X_{21}^{(2)}, X_{13}^{(2)}\right) \subset k^{18} \\
\mathbf{X} & =\left\{X_{11}^{(1)}, X_{11}^{(2)}, X_{22}^{(1)}, X_{22}^{(2)}, X_{12}^{(1)}, X_{12}^{(2)}, X_{33}^{(1)}, X_{21}^{(2)}, X_{13}^{(2)}\right\}, \\
\mathrm{e} & \\
W_{113} & =V\left(X_{11}^{(1)}, X_{11}^{(2)}, X_{22}^{(1)}, X_{22}^{(2)}, X_{12}^{(1)}, X_{12}^{(2)}, X_{33}^{(1)}, p_{32}^{\mathbf{x}}, p_{33}^{\mathbf{X}}, p_{34}^{\mathbf{X}}, X_{32}^{(2)}, X_{13}^{(2)}\right) \subset k^{18} \\
\mathbf{X} & =\left\{X_{11}^{(1)}, X_{11}^{(2)}, X_{22}^{(1)}, X_{22}^{(2)}, X_{12}^{(1)}, X_{12}^{(2)}, X_{33}^{(1)}, X_{32}^{(2)}, X_{13}^{(2)}\right\} .
\end{aligned}
$$

\section{Notemos que para}

$$
W_{111}=V\left(X_{11}^{(1)}, X_{11}^{(2)}, X_{22}^{(1)}, X_{22}^{(2)}, X_{12}^{(1)}, X_{12}^{(2)}, X_{33}^{(1)}, p_{32}^{\mathbf{X}}, p_{33}^{\mathbf{X}}, p_{34}^{\mathbf{x}}, X_{13}^{(1)}, X_{13}^{(2)}\right) \subset k^{18},
$$

as equações são

$$
\begin{aligned}
\mathbf{X} & =\left\{X_{11}^{(1)}, X_{11}^{(2)}, X_{22}^{(1)}, X_{22}^{(2)}, X_{12}^{(1)}, X_{12}^{(2)}, X_{33}^{(1)}, X_{13}^{(1)}, X_{13}^{(2)}\right\}, \\
p_{32}^{\mathbf{X}} & =X_{33}^{(2)}-X_{23}^{(1)} X_{32}^{(1)}-\underbrace{X_{13}^{(1)} X_{31}^{(1)}}_{=0} \\
& =X_{33}^{(2)}-X_{23}^{(1)} X_{32}^{(1)}, \\
p_{33}^{\mathbf{X}} & =\underbrace{X_{13}^{(1)} X_{21}^{(1)} X_{32}^{(1)}-X_{13}^{(1)} X_{31}^{(2)}}_{=0}-X_{23}^{(1)} X_{32}^{(2)}-X_{23}^{(2)} X_{32}^{(1)} \\
& =-X_{23}^{(1)} X_{32}^{(2)}-X_{23}^{(2)} X_{32}^{(1)}, \\
p_{34} \mathbf{x} & =-X_{23}^{(2)} X_{32}^{(2)}+\underbrace{X_{13}^{(1)} X_{21}^{(1)} X_{32}^{(2)}+X_{13}^{(1)} X_{21}^{(2)} X_{32}^{(1)}}_{=0} \\
& =-X_{23}^{(2)} X_{32}^{(2)}
\end{aligned}
$$

e portanto podemos obter a decomposição para $W_{111}$ da seguinte forma

$$
\begin{aligned}
W_{111}= & V\left(X_{11}^{(1)}, X_{11}^{(2)}, X_{22}^{(1)}, X_{22}^{(2)}, X_{12}^{(1)}, X_{12}^{(2)}, X_{33}^{(1)}, p_{32}^{\mathbf{x}}, p_{33}^{\mathbf{x}}, p_{34}^{\mathbf{x}}, X_{13}^{(1)}, X_{13}^{(2)}\right) \\
= & V\left(X_{11}^{(1)}, X_{11}^{(2)}, X_{22}^{(1)}, X_{22}^{(2)}, X_{12}^{(1)}, X_{12}^{(2)}, X_{33}^{(1)}, X_{33}^{(2)}-X_{23}^{(1)} X_{32}^{(1)}, X_{23}^{(1)} X_{32}^{(2)}, X_{23}^{(2)}, X_{13}^{(1)}, X_{13}^{(2)}\right) \cup \\
& \cup V\left(X_{11}^{(1)}, X_{11}^{(2)}, X_{22}^{(1)}, X_{22}^{(2)}, X_{12}^{(1)}, X_{12}^{(2)}, X_{33}^{(1)}, X_{33}^{(2)}-X_{23}^{(1)} X_{32}^{(1)}, X_{23}^{(2)} X_{32}^{(1)}, X_{32}^{(2)}, X_{13}^{(1)}, X_{13}^{(2)}\right) \\
= & V\left(X_{11}^{(1)}, X_{11}^{(2)}, X_{22}^{(1)}, X_{22}^{(2)}, X_{12}^{(1)}, X_{12}^{(2)}, X_{33}^{(1)}, X_{33}^{(2)}, X_{23}^{(1)}, X_{23}^{(2)}, X_{13}^{(1)}, X_{13}^{(2)}\right) \cup \\
& \cup V\left(X_{11}^{(1)}, X_{11}^{(2)}, X_{22}^{(1)}, X_{22}^{(2)}, X_{12}^{(1)}, X_{12}^{(2)}, X_{33}^{(1)}, X_{33}^{(2)}-X_{23}^{(1)} X_{32}^{(1)}, X_{32}^{(2)}, X_{23}^{(2)}, X_{13}^{(1)}, X_{13}^{(2)}\right) \cup \\
& \cup V\left(X_{11}^{(1)}, X_{11}^{(2)}, X_{22}^{(1)}, X_{22}^{(2)}, X_{12}^{(1)}, X_{12}^{(2)}, X_{33}^{(1)}, X_{33}^{(2)}, X_{32}^{(1)}, X_{32}^{(2)}, X_{13}^{(1)}, X_{13}^{(2)}\right) .
\end{aligned}
$$




\section{Denotando por}

$$
\begin{aligned}
& C_{1}:=V\left(X_{11}^{(1)}, X_{11}^{(2)}, X_{22}^{(1)}, X_{22}^{(2)}, X_{12}^{(1)}, X_{12}^{(2)}, X_{33}^{(1)}, X_{33}^{(2)}, X_{23}^{(1)}, X_{23}^{(2)}, X_{13}^{(1)}, X_{13}^{(2)}\right), \\
& C_{2}:=V\left(X_{11}^{(1)}, X_{11}^{(2)}, X_{22}^{(1)}, X_{22}^{(2)}, X_{12}^{(1)}, X_{12}^{(2)}, X_{33}^{(1)}, X_{33}^{(2)}-X_{23}^{(1)} X_{32}^{(1)}, X_{32}^{(2)}, X_{23}^{(2)}, X_{13}^{(1)}, X_{13}^{(2)}\right), \\
& C_{3}:=V\left(X_{11}^{(1)}, X_{11}^{(2)}, X_{22}^{(1)}, X_{22}^{(2)}, X_{12}^{(1)}, X_{12}^{(2)}, X_{33}^{(1)}, X_{33}^{(2)}, X_{32}^{(1)}, X_{32}^{(2)}, X_{13}^{(1)}, X_{13}^{(2)}\right),
\end{aligned}
$$

tem-se

$$
W_{111}=C_{1} \cup C_{2} \cup C_{3} .
$$

\section{Agora, notemos que para}

$$
W_{112}=V\left(X_{11}^{(1)}, X_{11}^{(2)}, X_{22}^{(1)}, X_{22}^{(2)}, X_{12}^{(1)}, X_{12}^{(2)}, X_{33}^{(1)}, p_{32}^{\mathbf{X}}, p_{33}^{\mathbf{X}}, p_{34}^{\mathbf{X}}, X_{21}^{(2)}, X_{13}^{(2)}\right) \subset k^{18},
$$

as equações são

$$
\begin{aligned}
\mathbf{X} & =\left\{X_{11}^{(1)}, X_{11}^{(2)}, X_{22}^{(1)}, X_{22}^{(2)}, X_{12}^{(1)}, X_{12}^{(2)}, X_{33}^{(1)}, X_{21}^{(2)}, X_{13}^{(2)}\right\}, \\
p_{32}^{\mathbf{X}} & =X_{33}^{(2)}-X_{23}^{(1)} X_{32}^{(1)}-X_{13}^{(1)} X_{31}^{(1)}, \\
p_{33}^{\mathbf{X}} & =X_{13}^{(1)} X_{21}^{(1)} X_{32}^{(1)}-X_{13}^{(1)} X_{31}^{(2)}-X_{23}^{(1)} X_{32}^{(2)}-X_{23}^{(2)} X_{32}^{(1)}, \\
p_{34}^{\mathbf{X}} & =-X_{23}^{(2)} X_{32}^{(2)}+X_{13}^{(1)} X_{21}^{(1)} X_{32}^{(2)}+\underbrace{X_{13}^{(1)} X_{21}^{(2)} X_{32}^{(1)}}_{=0} \\
& =-X_{23}^{(2)} X_{32}^{(2)}+X_{13}^{(1)} X_{21}^{(1)} X_{32}^{(2)} \\
& =\left(X_{13}^{(1)} X_{21}^{(1)}-X_{23}^{(2)}\right) X_{32}^{(2)}
\end{aligned}
$$

e portanto obtemos a seguinte decomposição para $W_{112}$

$$
\begin{aligned}
W_{112}= & V\left(X_{11}^{(1)}, X_{11}^{(2)}, X_{22}^{(1)}, X_{22}^{(2)}, X_{12}^{(1)}, X_{12}^{(2)}, X_{33}^{(1)}, p_{32}^{\mathbf{X}}, p_{33}^{\mathbf{X}}, p_{34}^{\mathbf{X}}, X_{21}^{(2)}, X_{13}^{(2)}\right) \\
= & V\left(X_{11}^{(1)}, X_{11}^{(2)}, X_{22}^{(1)}, X_{22}^{(2)}, X_{12}^{(1)}, X_{12}^{(2)}, X_{33}^{(1)}, X_{33}^{(2)}-X_{23}^{(1)} X_{32}^{(1)}-X_{13}^{(1)} X_{31}^{(1)},\right. \\
& \left.-X_{23}^{(1)} X_{32}^{(2)}-X_{23}^{(2)} X_{32}^{(1)}+X_{13}^{(1)} X_{21}^{(1)} X_{32}^{(1)}-X_{13}^{(1)} X_{31}^{(2)},\left(-X_{23}^{(2)}+X_{13}^{(1)} X_{21}^{(1)}\right) X_{32}^{(2)}, X_{21}^{(2)}, X_{13}^{(2)}\right) \\
= & V\left(X_{11}^{(1)}, X_{11}^{(2)}, X_{22}^{(1)}, X_{22}^{(2)}, X_{12}^{(1)}, X_{12}^{(2)}, X_{33}^{(1)}, X_{33}^{(2)}-X_{23}^{(1)} X_{32}^{(1)}-X_{13}^{(1)} X_{31}^{(1)},\right. \\
& \left.-X_{23}^{(2)} X_{32}^{(1)}+X_{13}^{(1)} X_{21}^{(1)} X_{32}^{(1)}-X_{13}^{(1)} X_{31}^{(2)}, X_{32}^{(2)}, X_{21}^{(2)}, X_{13}^{(2)}\right) \cup \\
\cup & V\left(X_{11}^{(1)}, X_{11}^{(2)}, X_{22}^{(1)}, X_{22}^{(2)}, X_{12}^{(1)}, X_{12}^{(2)}, X_{33}^{(1)}, X_{33}^{(2)}-X_{23}^{(1)} X_{32}^{(1)}-X_{13}^{(1)} X_{31}^{(1)},\right. \\
& \left.\quad-X_{23}^{(1)} X_{32}^{(2)}-X_{13}^{(1)} X_{31}^{(2)},-X_{23}^{(2)}+X_{13}^{(1)} X_{21}^{(1)}, X_{21}^{(2)}, X_{13}^{(2)}\right) .
\end{aligned}
$$

\section{Denotemos por}

$$
\begin{aligned}
C_{4}:= & V\left(X_{11}^{(1)}, X_{11}^{(2)}, X_{22}^{(1)}, X_{22}^{(2)}, X_{12}^{(1)}, X_{12}^{(2)}, X_{33}^{(1)}, X_{33}^{(2)}-X_{23}^{(1)} X_{32}^{(1)}-X_{13}^{(1)} X_{31}^{(1)},\right. \\
& \left.-X_{23}^{(2)} X_{32}^{(1)}+X_{13}^{(1)} X_{21}^{(1)} X_{32}^{(1)}-X_{13}^{(1)} X_{31}^{(2)}, X_{32}^{(2)}, X_{21}^{(2)}, X_{13}^{(2)}\right), \\
C_{5}:= & V\left(X_{11}^{(1)}, X_{11}^{(2)}, X_{22}^{(1)}, X_{22}^{(2)}, X_{12}^{(1)}, X_{12}^{(2)}, X_{33}^{(1)}, X_{33}^{(2)}-X_{23}^{(1)} X_{32}^{(1)}-X_{13}^{(1)} X_{31}^{(1)},\right. \\
& \left.\quad-X_{23}^{(1)} X_{32}^{(2)}-X_{13}^{(1)} X_{31}^{(2)},-X_{23}^{(2)}+X_{13}^{(1)} X_{21}^{(1)}, X_{21}^{(2)}, X_{13}^{(2)}\right) .
\end{aligned}
$$




\section{Portanto}

$$
W_{112}=C_{4} \cup C_{5} .
$$

Novamente, notemos que para

$$
W_{113}=V\left(X_{11}^{(1)}, X_{11}^{(2)}, X_{22}^{(1)}, X_{22}^{(2)}, X_{12}^{(1)}, X_{12}^{(2)}, X_{33}^{(1)}, p_{32}^{\mathbf{x}}, p_{33}^{\mathbf{x}}, p_{34}^{\mathbf{x}}, X_{32}^{(2)}, X_{13}^{(2)}\right) \subset k^{18}
$$

as equações são:

$$
\begin{aligned}
\mathbf{X} & =\left\{X_{11}^{(1)}, X_{11}^{(2)}, X_{22}^{(1)}, X_{22}^{(2)}, X_{12}^{(1)}, X_{12}^{(2)}, X_{33}^{(1)}, X_{32}^{(2)}, X_{13}^{(2)}\right\}, \\
p_{32}^{\mathbf{X}} & =X_{33}^{(2)}-X_{23}^{(1)} X_{32}^{(1)}-X_{13}^{(1)} X_{31}^{(1)}, \\
p_{33}^{\mathbf{X}} & =X_{13}^{(1)} X_{21}^{(1)} X_{32}^{(1)}-X_{13}^{(1)} X_{31}^{(2)}-\underbrace{X_{23}^{(1)} X_{32}^{(2)}}_{=0}-X_{23}^{(2)} X_{32}^{(1)} \\
& =X_{13}^{(1)} X_{21}^{(1)} X_{32}^{(1)}-X_{13}^{(1)} X_{31}^{(2)}-X_{23}^{(2)} X_{32}^{(1)}, \\
p_{34}^{\mathbf{X}} & =\underbrace{-X_{23}^{(2)} X_{32}^{(2)}+X_{13}^{(1)} X_{21}^{(1)} X_{32}^{(2)}}_{=0}+X_{13}^{(1)} X_{21}^{(2)} X_{32}^{(1)} \\
& =X_{13}^{(1)} X_{21}^{(2)} X_{32}^{(1)},
\end{aligned}
$$

obtendo a seguinte decomposição para $W_{113}$

$$
\begin{aligned}
& W_{113}=V\left(X_{11}^{(1)}, X_{11}^{(2)}, X_{22}^{(1)}, X_{22}^{(2)}, X_{12}^{(1)}, X_{12}^{(2)}, X_{33}^{(1)}, p_{32}^{\mathbf{x}}, p_{33}^{\mathbf{X}}, p_{34}^{\mathbf{X}}, X_{32}^{(2)}, X_{13}^{(2)}\right) \\
& =V\left(X_{11}^{(1)}, X_{11}^{(2)}, X_{22}^{(1)}, X_{22}^{(2)}, X_{12}^{(1)}, X_{12}^{(2)}, X_{33}^{(1)}, X_{33}^{(2)}-X_{23}^{(1)} X_{32}^{(1)}-X_{13}^{(1)} X_{31}^{(1)}\right. \text {, } \\
& \left.-X_{23}^{(2)} X_{32}^{(1)}+X_{13}^{(1)} X_{21}^{(1)} X_{32}^{(1)}-X_{13}^{(1)} X_{31}^{(2)}, X_{13}^{(1)} X_{21}^{(2)} X_{32}^{(1)}, X_{32}^{(2)}, X_{13}^{(2)}\right) \\
& =V\left(X_{11}^{(1)}, X_{11}^{(2)}, X_{22}^{(1)}, X_{22}^{(2)}, X_{12}^{(1)}, X_{12}^{(2)}, X_{33}^{(1)}, X_{33}^{(2)}-X_{23}^{(1)} X_{32}^{(1)}, X_{23}^{(2)} X_{32}^{(1)}, X_{13}^{(1)}, X_{32}^{(2)}, X_{13}^{(2)}\right) \cup \\
& \cup V\left(X_{11}^{(1)}, X_{11}^{(2)}, X_{22}^{(1)}, X_{22}^{(2)}, X_{12}^{(1)}, X_{12}^{(2)}, X_{33}^{(1)}, X_{33}^{(2)}-X_{23}^{(1)} X_{32}^{(1)}-X_{13}^{(1)} X_{31}^{(1)}\right. \text {, } \\
& \left.-X_{23}^{(2)} X_{32}^{(1)}+X_{13}^{(1)} X_{21}^{(1)} X_{32}^{(1)}-X_{13}^{(1)} X_{31}^{(2)}, X_{21}^{(2)}, X_{32}^{(2)}, X_{13}^{(2)}\right) \cup \\
& \cup V\left(X_{11}^{(1)}, X_{11}^{(2)}, X_{22}^{(1)}, X_{22}^{(2)}, X_{12}^{(1)}, X_{12}^{(2)}, X_{33}^{(1)}, X_{33}^{(2)}-X_{13}^{(1)} X_{31}^{(1)}, X_{13}^{(1)} X_{31}^{(2)}, X_{32}^{(1)}, X_{32}^{(2)}, X_{13}^{(2)}\right) \\
& =V\left(X_{11}^{(1)}, X_{11}^{(2)}, X_{22}^{(1)}, X_{22}^{(2)}, X_{12}^{(1)}, X_{12}^{(2)}, X_{33}^{(1)}, X_{33}^{(2)}-X_{23}^{(1)} X_{32}^{(1)}, X_{23}^{(2)}, X_{13}^{(1)}, X_{32}^{(2)}, X_{13}^{(2)}\right) \cup \\
& \cup V\left(X_{11}^{(1)}, X_{11}^{(2)}, X_{22}^{(1)}, X_{22}^{(2)}, X_{12}^{(1)}, X_{12}^{(2)}, X_{33}^{(1)}, X_{33}^{(2)}, X_{32}^{(1)}, X_{13}^{(1)}, X_{32}^{(2)}, X_{13}^{(2)}\right) \cup \\
& \cup V\left(X_{11}^{(1)}, X_{11}^{(2)}, X_{22}^{(1)}, X_{22}^{(2)}, X_{12}^{(1)}, X_{12}^{(2)}, X_{33}^{(1)}, X_{33}^{(2)}-X_{23}^{(1)} X_{32}^{(1)}-X_{13}^{(1)} X_{31}^{(1)}\right. \text {, } \\
& \left.-X_{23}^{(2)} X_{32}^{(1)}+X_{13}^{(1)} X_{21}^{(1)} X_{32}^{(1)}-X_{13}^{(1)} X_{31}^{(2)}, X_{21}^{(2)}, X_{32}^{(2)}, X_{13}^{(2)}\right) \cup \\
& \cup V\left(X_{11}^{(1)}, X_{11}^{(2)}, X_{22}^{(1)}, X_{22}^{(2)}, X_{12}^{(1)}, X_{12}^{(2)}, X_{33}^{(1)}, X_{33}^{(2)}, X_{13}^{(1)}, X_{32}^{(1)}, X_{32}^{(2)}, X_{13}^{(2)}\right) \cup \\
& \cup V\left(X_{11}^{(1)}, X_{11}^{(2)}, X_{22}^{(1)}, X_{22}^{(2)}, X_{12}^{(1)}, X_{12}^{(2)}, X_{33}^{(1)}, X_{33}^{(2)}-X_{13}^{(1)} X_{31}^{(1)}, X_{31}^{(2)}, X_{32}^{(1)}, X_{32}^{(2)}, X_{13}^{(2)}\right) \\
& =V\left(X_{11}^{(1)}, X_{11}^{(2)}, X_{22}^{(1)}, X_{22}^{(2)}, X_{12}^{(1)}, X_{12}^{(2)}, X_{33}^{(1)}, X_{33}^{(2)}-X_{23}^{(1)} X_{32}^{(1)}, X_{23}^{(2)}, X_{13}^{(1)}, X_{32}^{(2)}, X_{13}^{(2)}\right) \cup \\
& \cup V\left(X_{11}^{(1)}, X_{11}^{(2)}, X_{22}^{(1)}, X_{22}^{(2)}, X_{12}^{(1)}, X_{12}^{(2)}, X_{33}^{(1)}, X_{33}^{(2)}, X_{32}^{(1)}, X_{13}^{(1)}, X_{32}^{(2)}, X_{13}^{(2)}\right) \cup
\end{aligned}
$$




$$
\begin{aligned}
& \cup V\left(X_{11}^{(1)}, X_{11}^{(2)}, X_{22}^{(1)}, X_{22}^{(2)}, X_{12}^{(1)}, X_{12}^{(2)}, X_{33}^{(1)}, X_{33}^{(2)}-X_{23}^{(1)} X_{32}^{(1)}-X_{13}^{(1)} X_{31}^{(1)}\right. \\
& \left.\quad-X_{23}^{(2)} X_{32}^{(1)}+X_{13}^{(1)} X_{21}^{(1)} X_{32}^{(1)}-X_{13}^{(1)} X_{31}^{(2)}, X_{21}^{(2)}, X_{32}^{(2)}, X_{13}^{(2)}\right) \cup \\
& \cup V\left(X_{11}^{(1)}, X_{11}^{(2)}, X_{22}^{(1)}, X_{22}^{(2)}, X_{12}^{(1)}, X_{12}^{(2)}, X_{33}^{(1)}, X_{33}^{(2)}-X_{13}^{(1)} X_{31}^{(1)}, X_{31}^{(2)}, X_{32}^{(1)}, X_{32}^{(2)}, X_{13}^{(2)}\right)
\end{aligned}
$$

Como

$$
\begin{aligned}
C_{2}= & V\left(X_{11}^{(1)}, X_{11}^{(2)}, X_{22}^{(1)}, X_{22}^{(2)}, X_{12}^{(1)}, X_{12}^{(2)}, X_{33}^{(1)}, X_{33}^{(2)}-X_{23}^{(1)} X_{32}^{(1)}, X_{23}^{(2)}, X_{13}^{(1)}, X_{32}^{(2)}, X_{13}^{(2)}\right), \\
C_{3}= & V\left(X_{11}^{(1)}, X_{11}^{(2)}, X_{22}^{(1)}, X_{22}^{(2)}, X_{12}^{(1)}, X_{12}^{(2)}, X_{33}^{(1)}, X_{33}^{(2)}, X_{32}^{(1)}, X_{13}^{(1)}, X_{32}^{(2)}, X_{13}^{(2)}\right) \\
C_{4}= & V\left(X_{11}^{(1)}, X_{11}^{(2)}, X_{22}^{(1)}, X_{22}^{(2)}, X_{12}^{(1)}, X_{12}^{(2)}, X_{33}^{(1)}, X_{33}^{(2)}-X_{23}^{(1)} X_{32}^{(1)}-X_{13}^{(1)} X_{31}^{(1)},\right. \\
& \left.\quad-X_{23}^{(2)} X_{32}^{(1)}+X_{13}^{(1)} X_{21}^{(1)} X_{32}^{(1)}-X_{13}^{(1)} X_{31}^{(2)}, X_{32}^{(2)}, X_{21}^{(2)}, X_{13}^{(2)}\right)
\end{aligned}
$$

e, denotando por

$$
C_{6}:=V\left(X_{11}^{(1)}, X_{11}^{(2)}, X_{22}^{(1)}, X_{22}^{(2)}, X_{12}^{(1)}, X_{12}^{(2)}, X_{33}^{(1)}, X_{33}^{(2)}-X_{13}^{(1)} X_{31}^{(1)}, X_{31}^{(2)}, X_{32}^{(1)}, X_{32}^{(2)}, X_{13}^{(2)}\right),
$$

tem-se

$$
W_{113}=C_{2} \cup C_{3} \cup C_{4} \cup C_{6} .
$$

Agora, das equações (B.1), (B.2) e (B.3), temos

$$
W_{11}=C_{1} \cup C_{2} \cup C_{3} \cup C_{4} \cup C_{5} \cup C_{6} .
$$

\section{B.2.2 Decomposição de $W_{12}$}

Continuaremos com o mesmo raciocinio feito para determinar a decomposição em componentes irredutíveis de $W_{11}$. Lembremos que

$$
W_{12}=V\left(X_{11}^{(1)}, X_{11}^{(2)}, X_{22}^{(1)}, p_{22}^{\mathbf{x}}, X_{12}^{(1)}, X_{12}^{(2)}, X_{33}^{(1)}, p_{32}^{\mathbf{x}}, p_{33}^{\mathbf{x}}, p_{34}^{\mathbf{x}}, p_{35}^{\mathbf{x}}, \frac{p_{36}^{\mathbf{x}}}{X_{13}^{(2)}}\right) \subset k^{18},
$$

com

$$
\begin{aligned}
\mathbf{X}= & \left\{X_{11}^{(1)}, X_{11}^{(2)}, X_{22}^{(1)}, X_{12}^{(1)}, X_{12}^{(2)}, X_{33}^{(1)}\right\}, \\
p_{22}^{\mathbf{X}}= & X_{22}^{(2)}-\underbrace{X_{12}^{(1)} X_{21}^{(1)}}_{=0} \\
= & X_{22}^{(2)}, \\
p_{32}^{\mathbf{X}}= & X_{33}^{(2)}-X_{23}^{(1)} X_{32}^{(1)}-X_{13}^{(1)} X_{31}^{(1)}, \\
p_{33}^{\mathbf{X}}= & \underbrace{X_{12}^{(1)} X_{23}^{(1)} X_{31}^{(1)}}_{=0}+X_{13}^{(1)} X_{21}^{(1)} X_{32}^{(1)}-X_{13}^{(1)} X_{31}^{(2)}-X_{23}^{(1)} X_{32}^{(2)}-X_{13}^{(2)} X_{31}^{(1)}-X_{23}^{(2)} X_{32}^{(1)} \\
= & X_{13}^{(1)} X_{21}^{(1)} X_{32}^{(1)}-X_{13}^{(1)} X_{31}^{(2)}-X_{23}^{(1)} X_{32}^{(2)}-X_{13}^{(2)} X_{31}^{(1)}-X_{23}^{(2)} X_{32}^{(1)}, \\
p_{34}^{\mathbf{X}}= & -X_{23}^{(2)} X_{32}^{(2)}-X_{13}^{(2)} X_{31}^{(2)}+\underbrace{X_{12}^{(1)} X_{23}^{(1)} X_{31}^{(2)}}_{=0}+X_{13}^{(1)} X_{21}^{(1)} X_{32}^{(2)}-\underbrace{X_{13}^{(1)} X_{22}^{(2)} X_{31}^{(1)}}_{=0}+ \\
& +\underbrace{X_{12}^{(1)} X_{23}^{(2)} X_{31}^{(1)}}_{=0}+X_{13}^{(1)} X_{21}^{(2)} X_{32}^{(1)}+X_{13}^{(2)} X_{21}^{(1)} X_{32}^{(1)} \\
= & -X_{23}^{(2)} X_{32}^{(2)}-X_{13}^{(2)} X_{31}^{(2)}+X_{13}^{(1)} X_{21}^{(1)} X_{32}^{(2)}+X_{13}^{(1)} X_{21}^{(2)} X_{32}^{(1)}+X_{13}^{(2)} X_{21}^{(1)} X_{32}^{(1)},
\end{aligned}
$$




$$
\begin{aligned}
p_{35}^{\mathbf{X}} & =\underbrace{-X_{13}^{(1)} X_{22}^{(2)} X_{31}^{(2)}+X_{12}^{(1)} X_{23}^{(2)} X_{31}^{(2)}}_{=0}+X_{13}^{(1)} X_{21}^{(2)} X_{32}^{(2)}+X_{13}^{(2)} X_{21}^{(1)} X_{32}^{(2)}-\underbrace{X_{13}^{(2)} X_{22}^{(2)} X_{31}^{(1)}}_{=0}+X_{13}^{(2)} X_{21}^{(2)} X_{32}^{(1)} \\
& =X_{13}^{(1)} X_{21}^{(2)} X_{32}^{(2)}+X_{13}^{(2)} X_{21}^{(1)} X_{32}^{(2)}+X_{13}^{(2)} X_{21}^{(2)} X_{32}^{(1)}, \\
\frac{p_{36}^{\mathbf{X}}}{X_{13}^{(2)}} & =X_{21}^{(2)} X_{32}^{(2)}-\underbrace{X_{22}^{(2)} X_{31}^{(2)}}_{=0} \\
& =X_{21}^{(2)} X_{32}^{(2)},
\end{aligned}
$$

segue da última equação que $W_{12}=W_{121} \cup W_{122}$, em que

$$
\begin{aligned}
W_{121} & =V\left(X_{11}^{(1)}, X_{11}^{(2)}, X_{22}^{(1)}, X_{22}^{(2)}, X_{12}^{(1)}, X_{12}^{(2)}, X_{33}^{(1)}, p_{32}^{\mathbf{x}}, p_{33}^{\mathbf{x}}, p_{34}^{\mathbf{x}}, p_{35}^{\mathbf{X}}, X_{21}^{(2)}\right) \subset k^{18} \\
\mathbf{X} & =\left\{X_{11}^{(1)}, X_{11}^{(2)}, X_{22}^{(1)}, X_{22}^{(2)}, X_{12}^{(1)}, X_{12}^{(2)}, X_{33}^{(1)}, X_{21}^{(2)}\right\} \\
\mathrm{e} & \\
W_{122} & =V\left(X_{11}^{(1)}, X_{11}^{(2)}, X_{22}^{(1)}, X_{22}^{(2)}, X_{12}^{(1)}, X_{12}^{(2)}, X_{33}^{(1)}, p_{32}^{\mathbf{X}}, p_{33}^{\mathbf{X}}, p_{34}^{\mathbf{X}}, p_{35}^{\mathbf{X}}, X_{32}^{(2)}\right) \subset k^{18} \\
\mathbf{X} & =\left\{X_{11}^{(1)}, X_{11}^{(2)}, X_{22}^{(1)}, X_{22}^{(2)}, X_{12}^{(1)}, X_{12}^{(2)}, X_{33}^{(1)}, X_{32}^{(2)}\right\} .
\end{aligned}
$$

\section{Notemos que as equações para}

$$
W_{121}=V\left(X_{11}^{(1)}, X_{11}^{(2)}, X_{22}^{(1)}, X_{22}^{(2)}, X_{12}^{(1)}, X_{12}^{(2)}, X_{33}^{(1)}, p_{32}^{\mathbf{X}}, p_{33}^{\mathbf{X}}, p_{34}^{\mathbf{X}}, p_{35}^{\mathbf{X}}, X_{21}^{(2)}\right) \subset k^{18}
$$

são:

$$
\begin{aligned}
\mathbf{X} & =\left\{X_{11}^{(1)}, X_{11}^{(2)}, X_{22}^{(1)}, X_{22}^{(2)}, X_{12}^{(1)}, X_{12}^{(2)}, X_{33}^{(1)}, X_{21}^{(2)}\right\} \\
p_{32}^{\mathbf{X}} & =X_{33}^{(2)}-X_{23}^{(1)} X_{32}^{(1)}-X_{13}^{(1)} X_{31}^{(1)}, \\
p_{33}^{\mathbf{X}} & =X_{13}^{(1)} X_{21}^{(1)} X_{32}^{(1)}-X_{13}^{(1)} X_{31}^{(2)}-X_{23}^{(1)} X_{32}^{(2)}-X_{13}^{(2)} X_{31}^{(1)}-X_{23}^{(2)} X_{32}^{(1)}, \\
p_{34}^{\mathbf{X}} & =-X_{23}^{(2)} X_{32}^{(2)}-X_{13}^{(2)} X_{31}^{(2)}+X_{13}^{(1)} X_{21}^{(1)} X_{32}^{(2)}+\underbrace{X_{13}^{(1)} X_{21}^{(2)} X_{32}^{(1)}}_{=0}+X_{13}^{(2)} X_{21}^{(1)} X_{32}^{(1)}, \\
& =-X_{23}^{(2)} X_{32}^{(2)}-X_{13}^{(2)} X_{31}^{(2)}+X_{13}^{(1)} X_{21}^{(1)} X_{32}^{(2)}+X_{13}^{(2)} X_{21}^{(1)} X_{32}^{(1)}, \\
p_{35}^{\mathbf{X}} & =\underbrace{X_{13}^{(1)} X_{21}^{(2)} X_{32}^{(2)}}_{=0}+X_{13}^{(2)} X_{21}^{(1)} X_{32}^{(2)}+\underbrace{X_{13}^{(2)} X_{21}^{(2)} X_{32}^{(1)}}_{=0} \\
& =X_{13}^{(2)} X_{21}^{(1)} X_{32}^{(2)}
\end{aligned}
$$

e portanto a decomposição para $W_{121}$ é

$$
\begin{array}{rl}
W_{121}= & V\left(X_{11}^{(1)}, X_{11}^{(2)}, X_{22}^{(1)}, X_{22}^{(2)}, X_{12}^{(1)}, X_{12}^{(2)}, X_{33}^{(1)}, p_{32}^{\mathbf{x}}, p_{33}^{\mathbf{x}}, p_{34}^{\mathbf{x}}, p_{35}^{\mathbf{x}}, X_{21}^{(2)}\right) \\
=V & V\left(X_{11}^{(1)}, X_{11}^{(2)}, X_{22}^{(1)}, X_{22}^{(2)}, X_{12}^{(1)}, X_{12}^{(2)}, X_{33}^{(1)}, X_{33}^{(2)}-X_{23}^{(1)} X_{32}^{(1)}-X_{13}^{(1)} X_{31}^{(1)},\right. \\
& -X_{23}^{(1)} X_{32}^{(2)}-X_{23}^{(2)} X_{32}^{(1)}+X_{13}^{(1)} X_{21}^{(1)} X_{32}^{(1)}-X_{13}^{(1)} X_{31}^{(2)}-X_{13}^{(2)} X_{31}^{(1)}, \\
& \left.\quad-X_{23}^{(2)} X_{32}^{(2)}+X_{13}^{(1)} X_{21}^{(1)} X_{32}^{(2)}+X_{13}^{(2)} X_{21}^{(1)} X_{32}^{(1)}-X_{13}^{(2)} X_{31}^{(2)}, X_{13}^{(2)} X_{21}^{(1)} X_{32}^{(2)}, X_{21}^{(2)}\right) \\
=V & \left(X_{11}^{(1)}, X_{11}^{(2)}, X_{22}^{(1)}, X_{22}^{(2)}, X_{12}^{(1)}, X_{12}^{(2)}, X_{33}^{(1)}, X_{33}^{(2)}-X_{23}^{(1)} X_{32}^{(1)}-X_{13}^{(1)} X_{31}^{(1)},\right. \\
& \left.\quad-X_{23}^{(1)} X_{32}^{(2)}-X_{23}^{(2)} X_{32}^{(1)}+X_{13}^{(1)} X_{21}^{(1)} X_{32}^{(1)}-X_{13}^{(1)} X_{31}^{(2)},-X_{23}^{(2)} X_{32}^{(2)}+X_{13}^{(1)} X_{21}^{(1)} X_{32}^{(2)}, X_{13}^{(2)}, X_{21}^{(2)}\right) \cup \\
\cup & V\left(X_{11}^{(1)}, X_{11}^{(2)}, X_{22}^{(1)}, X_{22}^{(2)}, X_{12}^{(1)}, X_{12}^{(2)}, X_{33}^{(1)}, X_{33}^{(2)}-X_{23}^{(1)} X_{32}^{(1)}-X_{13}^{(1)} X_{31}^{(1)},\right. \\
& \left.\quad-X_{23}^{(1)} X_{32}^{(2)}-X_{23}^{(2)} X_{32}^{(1)}-X_{13}^{(1)} X_{31}^{(2)}-X_{13}^{(2)} X_{31}^{(1)},-X_{23}^{(2)} X_{32}^{(2)}-X_{13}^{(2)} X_{31}^{(2)}, X_{21}^{(1)}, X_{21}^{(2)}\right) \cup
\end{array}
$$




$$
\begin{aligned}
& \cup V\left(X_{11}^{(1)}, X_{11}^{(2)}, X_{22}^{(1)}, X_{22}^{(2)}, X_{12}^{(1)}, X_{12}^{(2)}, X_{33}^{(1)}, X_{33}^{(2)}-X_{23}^{(1)} X_{32}^{(1)}-X_{13}^{(1)} X_{31}^{(1)}\right. \text {, } \\
& \left.-X_{23}^{(2)} X_{32}^{(1)}+X_{13}^{(1)} X_{21}^{(1)} X_{32}^{(1)}-X_{13}^{(1)} X_{31}^{(2)}-X_{13}^{(2)} X_{31}^{(1)}, X_{13}^{(2)} X_{21}^{(1)} X_{32}^{(1)}-X_{13}^{(2)} X_{31}^{(2)}, X_{32}^{(2)}, X_{21}^{(2)}\right) \\
& =V\left(X_{11}^{(1)}, X_{11}^{(2)}, X_{22}^{(1)}, X_{22}^{(2)}, X_{12}^{(1)}, X_{12}^{(2)}, X_{33}^{(1)}, X_{33}^{(2)}-X_{23}^{(1)} X_{32}^{(1)}-X_{13}^{(1)} X_{31}^{(1)}\right. \text {, } \\
& \left.-X_{23}^{(2)} X_{32}^{(1)}+X_{13}^{(1)} X_{21}^{(1)} X_{32}^{(1)}-X_{13}^{(1)} X_{31}^{(2)}, X_{32}^{(2)}, X_{13}^{(2)}, X_{21}^{(2)}\right) \cup \\
& \cup V\left(X_{11}^{(1)}, X_{11}^{(2)}, X_{22}^{(1)}, X_{22}^{(2)}, X_{12}^{(1)}, X_{12}^{(2)}, X_{33}^{(1)}, X_{33}^{(2)}-X_{23}^{(1)} X_{32}^{(1)}-X_{13}^{(1)} X_{31}^{(1)}\right. \text {, } \\
& \left.-X_{23}^{(1)} X_{32}^{(2)}-X_{13}^{(1)} X_{31}^{(2)},-X_{23}^{(2)}+X_{13}^{(1)} X_{21}^{(1)}, X_{13}^{(2)}, X_{21}^{(2)}\right) \cup \\
& \cup V\left(X_{11}^{(1)}, X_{11}^{(2)}, X_{22}^{(1)}, X_{22}^{(2)}, X_{12}^{(1)}, X_{12}^{(2)}, X_{33}^{(1)}, X_{33}^{(2)}-X_{23}^{(1)} X_{32}^{(1)}-X_{13}^{(1)} X_{31}^{(1)}\right. \text {, } \\
& \left.-X_{23}^{(1)} X_{32}^{(2)}-X_{23}^{(2)} X_{32}^{(1)}-X_{13}^{(1)} X_{31}^{(2)}-X_{13}^{(2)} X_{31}^{(1)},-X_{23}^{(2)} X_{32}^{(2)}-X_{13}^{(2)} X_{31}^{(2)}, X_{21}^{(1)}, X_{21}^{(2)}\right) \cup \\
& \cup V\left(X_{11}^{(1)}, X_{11}^{(2)}, X_{22}^{(1)}, X_{22}^{(2)}, X_{12}^{(1)}, X_{12}^{(2)}, X_{33}^{(1)}, X_{33}^{(2)}-X_{23}^{(1)} X_{32}^{(1)}-X_{13}^{(1)} X_{31}^{(1)}\right. \text {, } \\
& \left.-X_{23}^{(2)} X_{32}^{(1)}+X_{13}^{(1)} X_{21}^{(1)} X_{32}^{(1)}-X_{13}^{(1)} X_{31}^{(2)}, X_{13}^{(2)}, X_{32}^{(2)}, X_{21}^{(2)}\right) \cup \\
& \cup V\left(X_{11}^{(1)}, X_{11}^{(2)}, X_{22}^{(1)}, X_{22}^{(2)}, X_{12}^{(1)}, X_{12}^{(2)}, X_{33}^{(1)}, X_{33}^{(2)}-X_{23}^{(1)} X_{32}^{(1)}-X_{13}^{(1)} X_{31}^{(1)}\right. \text {, } \\
& \left.-X_{23}^{(2)} X_{32}^{(1)}-X_{13}^{(2)} X_{31}^{(1)}, X_{21}^{(1)} X_{32}^{(1)}-X_{31}^{(2)}, X_{32}^{(2)}, X_{21}^{(2)}\right) \\
& =V\left(X_{11}^{(1)}, X_{11}^{(2)}, X_{22}^{(1)}, X_{22}^{(2)}, X_{12}^{(1)}, X_{12}^{(2)}, X_{33}^{(1)}, X_{33}^{(2)}-X_{23}^{(1)} X_{32}^{(1)}-X_{13}^{(1)} X_{31}^{(1)}\right. \text {, } \\
& \left.-X_{23}^{(2)} X_{32}^{(1)}+X_{13}^{(1)} X_{21}^{(1)} X_{32}^{(1)}-X_{13}^{(1)} X_{31}^{(2)}, X_{32}^{(2)}, X_{13}^{(2)}, X_{21}^{(2)}\right) \cup \\
& \cup V\left(X_{11}^{(1)}, X_{11}^{(2)}, X_{22}^{(1)}, X_{22}^{(2)}, X_{12}^{(1)}, X_{12}^{(2)}, X_{33}^{(1)}, X_{33}^{(2)}-X_{23}^{(1)} X_{32}^{(1)}-X_{13}^{(1)} X_{31}^{(1)}\right. \text {, } \\
& \left.-X_{23}^{(1)} X_{32}^{(2)}-X_{13}^{(1)} X_{31}^{(2)},-X_{23}^{(2)}+X_{13}^{(1)} X_{21}^{(1)}, X_{13}^{(2)}, X_{21}^{(2)}\right) \cup \\
& \cup V\left(X_{11}^{(1)}, X_{11}^{(2)}, X_{22}^{(1)}, X_{22}^{(2)}, X_{12}^{(1)}, X_{12}^{(2)}, X_{33}^{(1)}, X_{33}^{(2)}-X_{23}^{(1)} X_{32}^{(1)}-X_{13}^{(1)} X_{31}^{(1)}\right. \text {, } \\
& \left.-X_{23}^{(1)} X_{32}^{(2)}-X_{23}^{(2)} X_{32}^{(1)}-X_{13}^{(1)} X_{31}^{(2)}-X_{13}^{(2)} X_{31}^{(1)},-X_{23}^{(2)} X_{32}^{(2)}-X_{13}^{(2)} X_{31}^{(2)}, X_{21}^{(1)}, X_{21}^{(2)}\right) \cup \\
& \cup V\left(X_{11}^{(1)}, X_{11}^{(2)}, X_{22}^{(1)}, X_{22}^{(2)}, X_{12}^{(1)}, X_{12}^{(2)}, X_{33}^{(1)}, X_{33}^{(2)}-X_{23}^{(1)} X_{32}^{(1)}-X_{13}^{(1)} X_{31}^{(1)}\right. \text {, } \\
& \left.-X_{23}^{(2)} X_{32}^{(1)}-X_{13}^{(2)} X_{31}^{(1)}, X_{21}^{(1)} X_{32}^{(1)}-X_{31}^{(2)}, X_{32}^{(2)}, X_{21}^{(2)}\right) .
\end{aligned}
$$

\section{Como}

$$
\begin{aligned}
C_{4}=V & \left(X_{11}^{(1)}, X_{11}^{(2)}, X_{22}^{(1)}, X_{22}^{(2)}, X_{12}^{(1)}, X_{12}^{(2)}, X_{33}^{(1)}, X_{33}^{(2)}-X_{23}^{(1)} X_{32}^{(1)}-X_{13}^{(1)} X_{31}^{(1)},\right. \\
& \left.-X_{23}^{(2)} X_{32}^{(1)}+X_{13}^{(1)} X_{21}^{(1)} X_{32}^{(1)}-X_{13}^{(1)} X_{31}^{(2)}, X_{32}^{(2)}, X_{13}^{(2)}, X_{21}^{(2)}\right), \\
C_{5}=V( & X_{11}^{(1)}, X_{11}^{(2)}, X_{22}^{(1)}, X_{22}^{(2)}, X_{12}^{(1)}, X_{12}^{(2)}, X_{33}^{(1)}, X_{33}^{(2)}-X_{23}^{(1)} X_{32}^{(1)}-X_{13}^{(1)} X_{31}^{(1)}, \\
& \left.-X_{23}^{(1)} X_{32}^{(2)}-X_{13}^{(1)} X_{31}^{(2)},-X_{23}^{(2)}+X_{13}^{(1)} X_{21}^{(1)}, X_{13}^{(2)}, X_{21}^{(2)}\right)
\end{aligned}
$$


e denotando

$$
\begin{aligned}
& C_{7}= V\left(X_{11}^{(1)}, X_{11}^{(2)}, X_{22}^{(1)}, X_{22}^{(2)}, X_{12}^{(1)}, X_{12}^{(2)}, X_{33}^{(1)}, X_{33}^{(2)}-X_{23}^{(1)} X_{32}^{(1)}-X_{13}^{(1)} X_{31}^{(1)},\right. \\
&\left.\quad-X_{23}^{(1)} X_{32}^{(2)}-X_{23}^{(2)} X_{32}^{(1)}-X_{13}^{(1)} X_{31}^{(2)}-X_{13}^{(2)} X_{31}^{(1)},-X_{23}^{(2)} X_{32}^{(2)}-X_{13}^{(2)} X_{31}^{(2)}, X_{21}^{(1)}, X_{21}^{(2)}\right), \\
& C_{8}=V\left(X_{11}^{(1)}, X_{11}^{(2)}, X_{22}^{(1)}, X_{22}^{(2)}, X_{12}^{(1)}, X_{12}^{(2)}, X_{33}^{(1)}, X_{33}^{(2)}-X_{23}^{(1)} X_{32}^{(1)}-X_{13}^{(1)} X_{31}^{(1)},\right. \\
&\left.-X_{23}^{(2)} X_{32}^{(1)}-X_{13}^{(2)} X_{31}^{(1)}, X_{21}^{(1)} X_{32}^{(1)}-X_{31}^{(2)}, X_{32}^{(2)}, X_{21}^{(2)}\right),
\end{aligned}
$$

segue que

$$
W_{121}=C_{4} \cup C_{5} \cup C_{7} \cup C_{8} .
$$

Por outro lado, as equações de

$$
W_{122}=V\left(X_{11}^{(1)}, X_{11}^{(2)}, X_{22}^{(1)}, X_{22}^{(2)}, X_{12}^{(1)}, X_{12}^{(2)}, X_{33}^{(1)}, p_{32}^{\mathbf{x}}, p_{33}^{\mathbf{x}}, p_{34}^{\mathbf{x}}, p_{35}^{\mathbf{x}}, X_{32}^{(2)}\right) \subset k^{18}
$$

são:

$$
\begin{aligned}
\mathbf{X} & =\left\{X_{11}^{(1)}, X_{11}^{(2)}, X_{22}^{(1)}, X_{22}^{(2)}, X_{12}^{(1)}, X_{12}^{(2)}, X_{33}^{(1)}, X_{32}^{(2)}\right\}, \\
p_{32}^{\mathbf{X}} & =X_{33}^{(2)}-X_{23}^{(1)} X_{32}^{(1)}-X_{13}^{(1)} X_{31}^{(1)}, \\
p_{33}^{\mathbf{X}} & =X_{13}^{(1)} X_{21}^{(1)} X_{32}^{(1)}-X_{13}^{(1)} X_{31}^{(2)}-\underbrace{X_{23}^{(1)} X_{32}^{(2)}}_{=0}-X_{13}^{(2)} X_{31}^{(1)}-X_{23}^{(2)} X_{32}^{(1)} \\
& =X_{13}^{(1)} X_{21}^{(1)} X_{32}^{(1)}-X_{13}^{(1)} X_{31}^{(2)}-X_{13}^{(2)} X_{31}^{(1)}-X_{23}^{(2)} X_{32}^{(1)}, \\
p_{34}^{\mathbf{X}} & =-\underbrace{X_{23}^{(2)} X_{32}^{(2)}}_{=0}-X_{13}^{(2)} X_{31}^{(2)}+\underbrace{X_{13}^{(1)} X_{21}^{(1)} X_{32}^{(2)}}_{=0}+X_{13}^{(1)} X_{21}^{(2)} X_{32}^{(1)}+X_{13}^{(2)} X_{21}^{(1)} X_{32}^{(1)} \\
& =-X_{13}^{(2)} X_{31}^{(2)}+X_{13}^{(1)} X_{21}^{(2)} X_{32}^{(1)}+X_{13}^{(2)} X_{21}^{(1)} X_{32}^{(1)}, \\
p_{35}^{\mathbf{x}} & =\underbrace{X_{13}^{(1)} X_{21}^{(2)} X_{32}^{(2)}+X_{13}^{(2)} X_{21}^{(1)} X_{32}^{(2)}+X_{13}^{(2)} X_{21}^{(2)} X_{32}^{(1)}}_{=0} \\
& =X_{13}^{(2)} X_{21}^{(2)} X_{32}^{(1)},
\end{aligned}
$$

obtendo a seguinte decomposição

$$
\begin{array}{rl}
W_{122}= & V\left(X_{11}^{(1)}, X_{11}^{(2)}, X_{22}^{(1)}, X_{22}^{(2)}, X_{12}^{(1)}, X_{12}^{(2)}, X_{33}^{(1)}, p_{32}^{\mathbf{x}}, p_{33}^{\mathbf{x}}, p_{34}^{\mathbf{X}}, p_{35}^{\mathbf{X}}, X_{32}^{(2)}\right) \\
=V & \left(X_{11}^{(1)}, X_{11}^{(2)}, X_{22}^{(1)}, X_{22}^{(2)}, X_{12}^{(1)}, X_{12}^{(2)}, X_{33}^{(1)}, X_{33}^{(2)}-X_{23}^{(1)} X_{32}^{(1)}-X_{13}^{(1)} X_{31}^{(1)},\right. \\
& -X_{23}^{(2)} X_{32}^{(1)}+X_{13}^{(1)} X_{21}^{(1)} X_{32}^{(1)}-X_{13}^{(1)} X_{31}^{(2)}-X_{13}^{(2)} X_{31}^{(1)}, \\
& \left.X_{13}^{(1)} X_{21}^{(2)} X_{32}^{(1)}+X_{13}^{(2)} X_{21}^{(1)} X_{32}^{(1)}-X_{13}^{(2)} X_{31}^{(2)}, X_{13}^{(2)} X_{21}^{(2)} X_{32}^{(1)}, X_{32}^{(2)}\right) \\
=V & V\left(X_{11}^{(1)}, X_{11}^{(2)}, X_{22}^{(1)}, X_{22}^{(2)}, X_{12}^{(1)}, X_{12}^{(2)}, X_{33}^{(1)}, X_{33}^{(2)}-X_{23}^{(1)} X_{32}^{(1)}-X_{13}^{(1)} X_{31}^{(1)},\right. \\
& \left.-X_{23}^{(2)} X_{32}^{(1)}+X_{13}^{(1)} X_{21}^{(1)} X_{32}^{(1)}-X_{13}^{(1)} X_{31}^{(2)}, X_{13}^{(1)} X_{21}^{(2)} X_{32}^{(1)}, X_{13}^{(2)}, X_{32}^{(2)}\right) \cup \\
\cup & V\left(X_{11}^{(1)}, X_{11}^{(2)}, X_{22}^{(1)}, X_{22}^{(2)}, X_{12}^{(1)}, X_{12}^{(2)}, X_{33}^{(1)}, X_{33}^{(2)}-X_{23}^{(1)} X_{32}^{(1)}-X_{13}^{(1)} X_{31}^{(1)},\right. \\
& \left.-X_{23}^{(2)} X_{32}^{(1)}+X_{13}^{(1)} X_{21}^{(1)} X_{32}^{(1)}-X_{13}^{(1)} X_{31}^{(2)}-X_{13}^{(2)} X_{31}^{(1)}, X_{13}^{(2)} X_{21}^{(1)} X_{32}^{(1)}-X_{13}^{(2)} X_{31}^{(2)}, X_{21}^{(2)}, X_{32}^{(2)}\right) \cup \\
\cup & V\left(X_{11}^{(1)}, X_{11}^{(2)}, X_{22}^{(1)}, X_{22}^{(2)}, X_{12}^{(1)}, X_{12}^{(2)}, X_{33}^{(1)}, X_{33}^{(2)}-X_{13}^{(1)} X_{31}^{(1)}, X_{13}^{(1)} X_{31}^{(2)}+X_{13}^{(2)} X_{31}^{(1)}, X_{13}^{(2)} X_{31}^{(2)}, X_{32}^{(1)}, X_{32}^{(2)}\right)
\end{array}
$$




$$
\begin{aligned}
& =V\left(X_{11}^{(1)}, X_{11}^{(2)}, X_{22}^{(1)}, X_{22}^{(2)}, X_{12}^{(1)}, X_{12}^{(2)}, X_{33}^{(1)}, X_{33}^{(2)}-X_{23}^{(1)} X_{32}^{(1)}, X_{23}^{(2)} X_{32}^{(1)}, X_{13}^{(1)}, X_{13}^{(2)}, X_{32}^{(2)}\right) \cup \\
& \cup V\left(X_{11}^{(1)}, X_{11}^{(2)}, X_{22}^{(1)}, X_{22}^{(2)}, X_{12}^{(1)}, X_{12}^{(2)}, X_{33}^{(1)}, X_{33}^{(2)}-X_{23}^{(1)} X_{32}^{(1)}-X_{13}^{(1)} X_{31}^{(1)}\right. \text {, } \\
& \left.-X_{23}^{(2)} X_{32}^{(1)}+X_{13}^{(1)} X_{21}^{(1)} X_{32}^{(1)}-X_{13}^{(1)} X_{31}^{(2)}, X_{21}^{(2)}, X_{13}^{(2)}, X_{32}^{(2)}\right) \cup \\
& \cup V\left(X_{11}^{(1)}, X_{11}^{(2)}, X_{22}^{(1)}, X_{22}^{(2)}, X_{12}^{(1)}, X_{12}^{(2)}, X_{33}^{(1)}, X_{33}^{(2)}-X_{13}^{(1)} X_{31}^{(1)}, X_{13}^{(1)} X_{31}^{(2)}, X_{32}^{(1)}, X_{13}^{(2)}, X_{32}^{(2)}\right) \cup \\
& \cup V\left(X_{11}^{(1)}, X_{11}^{(2)}, X_{22}^{(1)}, X_{22}^{(2)}, X_{12}^{(1)}, X_{12}^{(2)}, X_{33}^{(1)}, X_{33}^{(2)}-X_{23}^{(1)} X_{32}^{(1)}-X_{13}^{(1)} X_{31}^{(1)}\right. \text {, } \\
& \left.-X_{23}^{(2)} X_{32}^{(1)}+X_{13}^{(1)} X_{21}^{(1)} X_{32}^{(1)}-X_{13}^{(1)} X_{31}^{(2)}, X_{13}^{(2)}, X_{21}^{(2)}, X_{32}^{(2)}\right) \cup \\
& \cup V\left(X_{11}^{(1)}, X_{11}^{(2)}, X_{22}^{(1)}, X_{22}^{(2)}, X_{12}^{(1)}, X_{12}^{(2)}, X_{33}^{(1)}, X_{33}^{(2)}-X_{23}^{(1)} X_{32}^{(1)}-X_{13}^{(1)} X_{31}^{(1)}\right. \text {, } \\
& \left.X_{23}^{(2)} X_{32}^{(1)}+X_{13}^{(2)} X_{31}^{(1)}, X_{21}^{(1)} X_{32}^{(1)}-X_{31}^{(2)}, X_{21}^{(2)}, X_{32}^{(2)}\right) \cup \\
& \cup V\left(X_{11}^{(1)}, X_{11}^{(2)}, X_{22}^{(1)}, X_{22}^{(2)}, X_{12}^{(1)}, X_{12}^{(2)}, X_{33}^{(1)}, X_{33}^{(2)}-X_{13}^{(1)} X_{31}^{(1)}, X_{13}^{(1)} X_{31}^{(2)}, X_{13}^{(2)}, X_{32}^{(1)}, X_{32}^{(2)}\right) \cup \\
& \cup V\left(X_{11}^{(1)}, X_{11}^{(2)}, X_{22}^{(1)}, X_{22}^{(2)}, X_{12}^{(1)}, X_{12}^{(2)}, X_{33}^{(1)}, X_{33}^{(2)}-X_{13}^{(1)} X_{31}^{(1)}, X_{13}^{(2)} X_{31}^{(1)}, X_{31}^{(2)}, X_{32}^{(1)}, X_{32}^{(2)}\right) \\
& =V\left(X_{11}^{(1)}, X_{11}^{(2)}, X_{22}^{(1)}, X_{22}^{(2)}, X_{12}^{(1)}, X_{12}^{(2)}, X_{33}^{(1)}, X_{33}^{(2)}-X_{23}^{(1)} X_{32}^{(1)}, X_{23}^{(2)} X_{32}^{(1)}, X_{13}^{(1)}, X_{13}^{(2)}, X_{32}^{(2)}\right) \cup \\
& \cup V\left(X_{11}^{(1)}, X_{11}^{(2)}, X_{22}^{(1)}, X_{22}^{(2)}, X_{12}^{(1)}, X_{12}^{(2)}, X_{33}^{(1)}, X_{33}^{(2)}-X_{23}^{(1)} X_{32}^{(1)}-X_{13}^{(1)} X_{31}^{(1)}\right. \text {, } \\
& \left.-X_{23}^{(2)} X_{32}^{(1)}+X_{13}^{(1)} X_{21}^{(1)} X_{32}^{(1)}-X_{13}^{(1)} X_{31}^{(2)}, X_{21}^{(2)}, X_{13}^{(2)}, X_{32}^{(2)}\right) \cup \\
& \cup V\left(X_{11}^{(1)}, X_{11}^{(2)}, X_{22}^{(1)}, X_{22}^{(2)}, X_{12}^{(1)}, X_{12}^{(2)}, X_{33}^{(1)}, X_{33}^{(2)}-X_{13}^{(1)} X_{31}^{(1)}, X_{13}^{(1)} X_{31}^{(2)}, X_{32}^{(1)}, X_{13}^{(2)}, X_{32}^{(2)}\right) \cup \\
& \cup V\left(X_{11}^{(1)}, X_{11}^{(2)}, X_{22}^{(1)}, X_{22}^{(2)}, X_{12}^{(1)}, X_{12}^{(2)}, X_{33}^{(1)}, X_{33}^{(2)}-X_{23}^{(1)} X_{32}^{(1)}-X_{13}^{(1)} X_{31}^{(1)}\right. \text {, } \\
& \left.X_{23}^{(2)} X_{32}^{(1)}+X_{13}^{(2)} X_{31}^{(1)}, X_{21}^{(1)} X_{32}^{(1)}-X_{31}^{(2)}, X_{21}^{(2)}, X_{32}^{(2)}\right) \cup \\
& \cup V\left(X_{11}^{(1)}, X_{11}^{(2)}, X_{22}^{(1)}, X_{22}^{(2)}, X_{12}^{(1)}, X_{12}^{(2)}, X_{33}^{(1)}, X_{33}^{(2)}-X_{13}^{(1)} X_{31}^{(1)}, X_{13}^{(2)} X_{31}^{(1)}, X_{31}^{(2)}, X_{32}^{(1)}, X_{32}^{(2)}\right) \\
& =V\left(X_{11}^{(1)}, X_{11}^{(2)}, X_{22}^{(1)}, X_{22}^{(2)}, X_{12}^{(1)}, X_{12}^{(2)}, X_{33}^{(1)}, X_{33}^{(2)}-X_{23}^{(1)} X_{32}^{(1)}, X_{23}^{(2)}, X_{13}^{(1)}, X_{13}^{(2)}, X_{32}^{(2)}\right) \cup \\
& \cup V\left(X_{11}^{(1)}, X_{11}^{(2)}, X_{22}^{(1)}, X_{22}^{(2)}, X_{12}^{(1)}, X_{12}^{(2)}, X_{33}^{(1)}, X_{33}^{(2)}, X_{32}^{(1)}, X_{13}^{(1)}, X_{13}^{(2)}, X_{32}^{(2)}\right) \cup \\
& \cup V\left(X_{11}^{(1)}, X_{11}^{(2)}, X_{22}^{(1)}, X_{22}^{(2)}, X_{12}^{(1)}, X_{12}^{(2)}, X_{33}^{(1)}, X_{33}^{(2)}-X_{23}^{(1)} X_{32}^{(1)}-X_{13}^{(1)} X_{31}^{(1)}\right. \text {, } \\
& \left.-X_{23}^{(2)} X_{32}^{(1)}+X_{13}^{(1)} X_{21}^{(1)} X_{32}^{(1)}-X_{13}^{(1)} X_{31}^{(2)}, X_{21}^{(2)}, X_{13}^{(2)}, X_{32}^{(2)}\right) \cup \\
& \cup V\left(X_{11}^{(1)}, X_{11}^{(2)}, X_{22}^{(1)}, X_{22}^{(2)}, X_{12}^{(1)}, X_{12}^{(2)}, X_{33}^{(1)}, X_{33}^{(2)}, X_{13}^{(1)}, X_{32}^{(1)}, X_{13}^{(2)}, X_{32}^{(2)}\right) \cup \\
& \cup V\left(X_{11}^{(1)}, X_{11}^{(2)}, X_{22}^{(1)}, X_{22}^{(2)}, X_{12}^{(1)}, X_{12}^{(2)}, X_{33}^{(1)}, X_{33}^{(2)}-X_{13}^{(1)} X_{31}^{(1)}, X_{31}^{(2)}, X_{32}^{(1)}, X_{13}^{(2)}, X_{32}^{(2)}\right) \cup \\
& \cup V\left(X_{11}^{(1)}, X_{11}^{(2)}, X_{22}^{(1)}, X_{22}^{(2)}, X_{12}^{(1)}, X_{12}^{(2)}, X_{33}^{(1)}, X_{33}^{(2)}-X_{23}^{(1)} X_{32}^{(1)}-X_{13}^{(1)} X_{31}^{(1)}\right. \text {, } \\
& \left.X_{23}^{(2)} X_{32}^{(1)}+X_{13}^{(2)} X_{31}^{(1)}, X_{21}^{(1)} X_{32}^{(1)}-X_{31}^{(2)}, X_{21}^{(2)}, X_{32}^{(2)}\right) \cup \\
& \cup V\left(X_{11}^{(1)}, X_{11}^{(2)}, X_{22}^{(1)}, X_{22}^{(2)}, X_{12}^{(1)}, X_{12}^{(2)}, X_{33}^{(1)}, X_{33}^{(2)}-X_{13}^{(1)} X_{31}^{(1)}, X_{13}^{(2)}, X_{31}^{(2)}, X_{32}^{(1)}, X_{32}^{(2)}\right) \cup
\end{aligned}
$$




$$
\begin{aligned}
& \cup V\left(X_{11}^{(1)}, X_{11}^{(2)}, X_{22}^{(1)}, X_{22}^{(2)}, X_{12}^{(1)}, X_{12}^{(2)}, X_{33}^{(1)}, X_{33}^{(2)}, X_{31}^{(1)}, X_{31}^{(2)}, X_{32}^{(1)}, X_{32}^{(2)}\right) \\
= & V\left(X_{11}^{(1)}, X_{11}^{(2)}, X_{22}^{(1)}, X_{22}^{(2)}, X_{12}^{(1)}, X_{12}^{(2)}, X_{33}^{(1)}, X_{33}^{(2)}-X_{23}^{(1)} X_{32}^{(1)}, X_{23}^{(2)}, X_{13}^{(1)}, X_{13}^{(2)}, X_{32}^{(2)}\right) \cup \\
\cup & V\left(X_{11}^{(1)}, X_{11}^{(2)}, X_{22}^{(1)}, X_{22}^{(2)}, X_{12}^{(1)}, X_{12}^{(2)}, X_{33}^{(1)}, X_{33}^{(2)}, X_{32}^{(1)}, X_{13}^{(1)}, X_{13}^{(2)}, X_{32}^{(2)}\right) \cup \\
\cup & V\left(X_{11}^{(1)}, X_{11}^{(2)}, X_{22}^{(1)}, X_{22}^{(2)}, X_{12}^{(1)}, X_{12}^{(2)}, X_{33}^{(1)}, X_{33}^{(2)}-X_{23}^{(1)} X_{32}^{(1)}-X_{13}^{(1)} X_{31}^{(1)},\right. \\
& \left.-X_{23}^{(2)} X_{32}^{(1)}+X_{13}^{(1)} X_{21}^{(1)} X_{32}^{(1)}-X_{13}^{(1)} X_{31}^{(2)}, X_{21}^{(2)}, X_{13}^{(2)}, X_{32}^{(2)}\right) \cup \\
\cup & V\left(X_{11}^{(1)}, X_{11}^{(2)}, X_{22}^{(1)}, X_{22}^{(2)}, X_{12}^{(1)}, X_{12}^{(2)}, X_{33}^{(1)}, X_{33}^{(2)}-X_{13}^{(1)} X_{31}^{(1)}, X_{31}^{(2)}, X_{32}^{(1)}, X_{13}^{(2)}, X_{32}^{(2)}\right) \cup \\
\cup & V\left(X_{11}^{(1)}, X_{11}^{(2)}, X_{22}^{(1)}, X_{22}^{(2)}, X_{12}^{(1)}, X_{12}^{(2)}, X_{33}^{(1)}, X_{33}^{(2)}-X_{23}^{(1)} X_{32}^{(1)}-X_{13}^{(1)} X_{31}^{(1)},\right. \\
& \left.X_{23}^{(2)} X_{32}^{(1)}+X_{13}^{(2)} X_{31}^{(1)}, X_{21}^{(1)} X_{32}^{(1)}-X_{31}^{(2)}, X_{21}^{(2)}, X_{32}^{(2)}\right) \cup \\
\cup & V\left(X_{11}^{(1)}, X_{11}^{(2)}, X_{22}^{(1)}, X_{22}^{(2)}, X_{12}^{(1)}, X_{12}^{(2)}, X_{33}^{(1)}, X_{33}^{(2)}, X_{31}^{(1)}, X_{31}^{(2)}, X_{32}^{(1)}, X_{32}^{(2)}\right) .
\end{aligned}
$$

Como

$$
\begin{aligned}
& C_{2}= V\left(X_{11}^{(1)}, X_{11}^{(2)}, X_{22}^{(1)}, X_{22}^{(2)}, X_{12}^{(1)}, X_{12}^{(2)}, X_{33}^{(1)}, X_{33}^{(2)}-X_{23}^{(1)} X_{32}^{(1)}, X_{23}^{(2)}, X_{13}^{(1)}, X_{13}^{(2)}, X_{32}^{(2)}\right), \\
& C_{3}=V\left(X_{11}^{(1)}, X_{11}^{(2)}, X_{22}^{(1)}, X_{22}^{(2)}, X_{12}^{(1)}, X_{12}^{(2)}, X_{33}^{(1)}, X_{33}^{(2)}, X_{32}^{(1)}, X_{13}^{(1)}, X_{13}^{(2)}, X_{32}^{(2)}\right), \\
& C_{4}=V\left(X_{11}^{(1)}, X_{11}^{(2)}, X_{22}^{(1)}, X_{22}^{(2)}, X_{12}^{(1)}, X_{12}^{(2)}, X_{33}^{(1)}, X_{33}^{(2)}-X_{23}^{(1)} X_{32}^{(1)}-X_{13}^{(1)} X_{31}^{(1)},\right. \\
&\left.\quad-X_{23}^{(2)} X_{32}^{(1)}+X_{13}^{(1)} X_{21}^{(1)} X_{32}^{(1)}-X_{13}^{(1)} X_{31}^{(2)}, X_{21}^{(2)}, X_{13}^{(2)}, X_{32}^{(2)}\right), \\
& C_{6}=V\left(X_{11}^{(1)}, X_{11}^{(2)}, X_{22}^{(1)}, X_{22}^{(2)}, X_{12}^{(1)}, X_{12}^{(2)}, X_{33}^{(1)}, X_{33}^{(2)}-X_{13}^{(1)} X_{31}^{(1)}, X_{31}^{(2)}, X_{32}^{(1)}, X_{13}^{(2)}, X_{32}^{(2)}\right), \\
& C_{8}=V\left(X_{11}^{(1)}, X_{11}^{(2)}, X_{22}^{(1)}, X_{22}^{(2)}, X_{12}^{(1)}, X_{12}^{(2)}, X_{33}^{(1)}, X_{33}^{(2)}-X_{23}^{(1)} X_{32}^{(1)}-X_{13}^{(1)} X_{31}^{(1)},\right. \\
&\left.X_{23}^{(2)} X_{32}^{(1)}+X_{13}^{(2)} X_{31}^{(1)}, X_{21}^{(1)} X_{32}^{(1)}-X_{31}^{(2)}, X_{21}^{(2)}, X_{32}^{(2)}\right)
\end{aligned}
$$

e denotando

$$
C_{9}:=V\left(X_{11}^{(1)}, X_{11}^{(2)}, X_{22}^{(1)}, X_{22}^{(2)}, X_{12}^{(1)}, X_{12}^{(2)}, X_{33}^{(1)}, X_{33}^{(2)}, X_{31}^{(1)}, X_{31}^{(2)}, X_{32}^{(1)}, X_{32}^{(2)}\right)
$$

tem-se

$$
W_{122}=C_{2} \cup C_{3} \cup C_{4} \cup C_{6} \cup C_{8} \cup C_{9} .
$$

Portanto, pelas equações (B.5) e (B.6)

$$
W_{12}=C_{2} \cup C_{3} \cup C_{4} \cup C_{5} \cup C_{6} \cup C_{7} \cup C_{8} \cup C_{9} .
$$

\section{B.2.3 Decomposição de $W_{13}$}

Continuando da mesma forma, para as variedades $W_{11}$ e $W_{12}$, as equações de

$$
W_{13}=V\left(X_{11}^{(1)}, X_{11}^{(2)}, X_{22}^{(1)}, p_{22}^{\mathbf{X}}, X_{21}^{(2)}, X_{12}^{(2)}, X_{33}^{(1)}, p_{32}^{\mathbf{X}}, p_{33}^{\mathbf{X}}, p_{34}^{\mathbf{X}}, p_{35}^{\mathbf{X}}, X_{13}^{(2)}\right) \subset k^{18}
$$

são:

$$
\mathbf{X}=\left\{X_{11}^{(1)}, X_{11}^{(2)}, X_{22}^{(1)}, X_{21}^{(2)}, X_{12}^{(2)}, X_{33}^{(1)}, X_{13}^{(2)}\right\}
$$




$$
\begin{aligned}
p_{22}^{\mathbf{X}}= & X_{22}^{(2)}-X_{12}^{(1)} X_{21}^{(1)}, \\
p_{32}^{\mathbf{X}}= & X_{33}^{(2)}-X_{23}^{(1)} X_{32}^{(1)}-X_{13}^{(1)} X_{31}^{(1)}, \\
p_{33}^{\mathbf{X}}= & X_{12}^{(1)} X_{23}^{(1)} X_{31}^{(1)}+X_{13}^{(1)} X_{21}^{(1)} X_{32}^{(1)}-X_{13}^{(1)} X_{31}^{(2)}-X_{23}^{(1)} X_{32}^{(2)}-\underbrace{X_{13}^{(2)} X_{31}^{(1)}-X_{23}^{(2)} X_{32}^{(1)}}_{=0} \\
= & X_{12}^{(1)} X_{23}^{(1)} X_{31}^{(1)}+X_{13}^{(1)} X_{21}^{(1)} X_{32}^{(1)}-X_{13}^{(1)} X_{31}^{(2)}-X_{23}^{(1)} X_{32}^{(2)}-X_{23}^{(2)} X_{32}^{(1)}, \\
p_{34}^{\mathbf{X}}= & -X_{23}^{(2)} X_{32}^{(2)}-\underbrace{X_{13}^{(2)} X_{31}^{(2)}+X_{12}^{(1)} X_{23}^{(1)} X_{31}^{(2)}+X_{13}^{(1)} X_{21}^{(1)} X_{32}^{(2)}-X_{13}^{(1)} X_{22}^{(2)} X_{31}^{(1)}+}_{=0} \\
& +X_{12}^{(1)} X_{23}^{(2)} X_{31}^{(1)}+\underbrace{X_{13}^{(1)} X_{21}^{(2)} X_{32}^{(1)}+X_{13}^{(2)} X_{21}^{(1)} X_{32}^{(1)}}_{=0} \\
= & -X_{23}^{(2)} X_{32}^{(2)}+X_{12}^{(1)} X_{23}^{(1)} X_{31}^{(2)}+X_{13}^{(1)} X_{21}^{(1)} X_{32}^{(2)}-X_{13}^{(1)} X_{22}^{(2)} X_{31}^{(1)}+X_{12}^{(1)} X_{23}^{(2)} X_{31}^{(1)}, \\
p_{35}^{\mathbf{X}}= & -X_{13}^{(1)} X_{22}^{(2)} X_{31}^{(2)}+X_{12}^{(1)} X_{23}^{(2)} X_{31}^{(2)}+\underbrace{(1)}_{13} X_{21}^{(2)} X_{32}^{(2)}+X_{13}^{(2)} X_{21}^{(1)} X_{32}^{(2)}-X_{13}^{(2)} X_{22}^{(2)} X_{31}^{(1)}+X_{13}^{(2)} X_{21}^{(2)} X_{32}^{(1)} \\
= & -X_{13}^{(1)} X_{22}^{(2)} X_{31}^{(2)}+X_{12}^{(1)} X_{23}^{(2)} X_{31}^{(2)} \\
= & \left(X_{12}^{(1)} X_{23}^{(2)}-X_{13}^{(1)} X_{22}^{(2)}\right) X_{31}^{(2)},
\end{aligned}
$$

segue da última equação que $W_{13}=W_{131} \cup W_{132}$, com

$$
\begin{aligned}
W_{131} & =V\left(X_{11}^{(1)}, X_{11}^{(2)}, X_{22}^{(1)}, p_{22}^{\mathbf{X}}, X_{21}^{(2)}, X_{12}^{(2)}, X_{33}^{(1)}, p_{32}^{\mathbf{x}}, p_{33}^{\mathbf{x}}, p_{34}^{\mathbf{x}}, X_{31}^{(2)}, X_{13}^{(2)}\right) \subset k^{18} \\
\mathbf{X} & =\left\{X_{11}^{(1)}, X_{11}^{(2)}, X_{22}^{(1)}, X_{21}^{(2)}, X_{12}^{(2)}, X_{33}^{(1)}, X_{31}^{(2)}, X_{13}^{(2)}\right\} \\
\mathrm{e} & \\
W_{132} & =V\left(X_{11}^{(1)}, X_{11}^{(2)}, X_{22}^{(1)}, p_{22}^{\mathbf{X}}, X_{21}^{(2)}, X_{12}^{(2)}, X_{33}^{(1)}, p_{32}^{\mathbf{x}}, p_{33}^{\mathbf{x}}, p_{34}^{\mathbf{x}}, \frac{p_{35}^{\mathbf{X}}}{X_{31}^{(2)}}, X_{13}^{(2)}\right) \subset k^{18} \\
\mathbf{X} & =\left\{X_{11}^{(1)}, X_{11}^{(2)}, X_{22}^{(1)}, X_{21}^{(2)}, X_{12}^{(2)}, X_{33}^{(1)}, X_{13}^{(2)}\right\},
\end{aligned}
$$

reparando que $\operatorname{com} \frac{p_{35}^{\mathrm{X}}}{X_{31}^{(2)}}$, queremos dizer

$$
\frac{p_{35}^{\mathbf{X}}}{X_{31}^{(2)}}=X_{12}^{(1)} X_{23}^{(2)}-X_{13}^{(1)} X_{22}^{(2)} .
$$

Agora, as equações que determinam

$$
W_{131}=V\left(X_{11}^{(1)}, X_{11}^{(2)}, X_{22}^{(1)}, p_{22}^{\mathbf{x}}, X_{21}^{(2)}, X_{12}^{(2)}, X_{33}^{(1)}, p_{32}^{\mathbf{x}}, p_{33}^{\mathbf{x}}, p_{34}^{\mathbf{x}}, X_{31}^{(2)}, X_{13}^{(2)}\right) \subset k^{18}
$$

são:

$$
\begin{aligned}
\mathbf{X} & =\left\{X_{11}^{(1)}, X_{11}^{(2)}, X_{22}^{(1)}, X_{21}^{(2)}, X_{12}^{(2)}, X_{33}^{(1)}, X_{31}^{(2)}, X_{13}^{(2)}\right\} \\
p_{22}^{\mathbf{X}} & =X_{22}^{(2)}-X_{12}^{(1)} X_{21}^{(1)}, \\
p_{32}^{\mathbf{X}} & =X_{33}^{(2)}-X_{23}^{(1)} X_{32}^{(1)}-X_{13}^{(1)} X_{31}^{(1)}, \\
p_{33}^{\mathbf{X}} & =X_{12}^{(1)} X_{23}^{(1)} X_{31}^{(1)}+X_{13}^{(1)} X_{21}^{(1)} X_{32}^{(1)}-\underbrace{X_{13}^{(1)} X_{31}^{(2)}}_{=0}-X_{23}^{(1)} X_{32}^{(2)}-X_{23}^{(2)} X_{32}^{(1)} \\
& =X_{12}^{(1)} X_{23}^{(1)} X_{31}^{(1)}+X_{13}^{(1)} X_{21}^{(1)} X_{32}^{(1)}-X_{23}^{(1)} X_{32}^{(2)}-X_{23}^{(2)} X_{32}^{(1)} \\
& =X_{23}^{(1)}\left(X_{12}^{(1)} X_{31}^{(1)}-X_{32}^{(2)}\right)-\left(X_{23}^{(2)}-X_{13}^{(1)} X_{21}^{(1)}\right) X_{32}^{(1)}, \\
p_{34}^{\mathbf{X}} & =-X_{23}^{(2)} X_{32}^{(2)}+\underbrace{X_{12}^{(1)} X_{23}^{(1)} X_{31}^{(2)}}_{=0}+X_{13}^{(1)} X_{21}^{(1)} X_{32}^{(2)}-X_{13}^{(1)} X_{22}^{(2)} X_{31}^{(1)}+X_{12}^{(1)} X_{23}^{(2)} X_{31}^{(1)}
\end{aligned}
$$




$$
\begin{aligned}
& =-X_{23}^{(2)} X_{32}^{(2)}+X_{13}^{(1)} X_{21}^{(1)} X_{32}^{(2)}-X_{13}^{(1)} X_{22}^{(2)} X_{31}^{(1)}+X_{12}^{(1)} X_{23}^{(2)} X_{31}^{(1)} \\
& =-X_{23}^{(2)} X_{32}^{(2)}+X_{13}^{(1)} X_{21}^{(1)} X_{32}^{(2)}-X_{13}^{(1)} X_{12}^{(1)} X_{21}^{(1)} X_{31}^{(1)}+X_{12}^{(1)} X_{23}^{(2)} X_{31}^{(1)} \\
& =-\left(X_{23}^{(2)}-X_{13}^{(1)} X_{21}^{(1)}\right) X_{32}^{(2)}+\left(-X_{13}^{(1)} X_{21}^{(1)}+X_{23}^{(2)}\right) X_{12}^{(1)} X_{31}^{(1)} \\
& =\left(X_{23}^{(2)}-X_{13}^{(1)} X_{21}^{(1)}\right)\left(-X_{32}^{(2)}+X_{12}^{(1)} X_{31}^{(1)}\right) .
\end{aligned}
$$

\section{Portanto, tem-se a decomposição}

$$
\begin{aligned}
& W_{131}=V\left(X_{11}^{(1)}, X_{11}^{(2)}, X_{22}^{(1)}, p_{22}^{\mathbf{x}}, X_{21}^{(2)}, X_{12}^{(2)}, X_{33}^{(1)}, p_{32}^{\mathbf{x}}, p_{33}^{\mathbf{x}}, p_{34}^{\mathbf{x}}, X_{31}^{(2)}, X_{13}^{(2)}\right) \\
& =V\left(X_{11}^{(1)}, X_{11}^{(2)}, X_{22}^{(1)}, X_{22}^{(2)}-X_{12}^{(1)} X_{21}^{(1)}, X_{21}^{(2)}, X_{12}^{(2)}, X_{33}^{(1)}, X_{33}^{(2)}-X_{23}^{(1)} X_{32}^{(1)}-X_{13}^{(1)} X_{31}^{(1)}\right. \text {, } \\
& \left.X_{23}^{(1)}\left(X_{12}^{(1)} X_{31}^{(1)}-X_{32}^{(2)}\right)-\left(X_{23}^{(2)}-X_{13}^{(1)} X_{21}^{(1)}\right) X_{32}^{(1)},\left(X_{23}^{(2)}-X_{13}^{(1)} X_{21}^{(1)}\right)\left(-X_{32}^{(2)}+X_{12}^{(1)} X_{31}^{(1)}\right), X_{31}^{(2)}, X_{13}^{(2)}\right) \\
& =V\left(X_{11}^{(1)}, X_{11}^{(2)}, X_{22}^{(1)}, X_{22}^{(2)}-X_{12}^{(1)} X_{21}^{(1)}, X_{21}^{(2)}, X_{12}^{(2)}, X_{33}^{(1)}, X_{33}^{(2)}-X_{23}^{(1)} X_{32}^{(1)}-X_{13}^{(1)} X_{31}^{(1)}\right. \text {, } \\
& \left.X_{23}^{(1)}\left(X_{12}^{(1)} X_{31}^{(1)}-X_{32}^{(2)}\right), X_{23}^{(2)}-X_{13}^{(1)} X_{21}^{(1)}, X_{31}^{(2)}, X_{13}^{(2)}\right) \cup \\
& \cup V\left(X_{11}^{(1)}, X_{11}^{(2)}, X_{22}^{(1)}, X_{22}^{(2)}-X_{12}^{(1)} X_{21}^{(1)}, X_{21}^{(2)}, X_{12}^{(2)}, X_{33}^{(1)}, X_{33}^{(2)}-X_{23}^{(1)} X_{32}^{(1)}-X_{13}^{(1)} X_{31}^{(1)}\right. \text {, } \\
& \left.\left(X_{23}^{(2)}-X_{13}^{(1)} X_{21}^{(1)}\right) X_{32}^{(1)},-X_{32}^{(2)}+X_{12}^{(1)} X_{31}^{(1)}, X_{31}^{(2)}, X_{13}^{(2)}\right) \\
& =V\left(X_{11}^{(1)}, X_{11}^{(2)}, X_{22}^{(1)}, X_{22}^{(2)}-X_{12}^{(1)} X_{21}^{(1)}, X_{21}^{(2)}, X_{12}^{(2)}, X_{33}^{(1)}, X_{33}^{(2)}-X_{13}^{(1)} X_{31}^{(1)}, X_{23}^{(1)}, X_{23}^{(2)}-X_{13}^{(1)} X_{21}^{(1)}, X_{31}^{(2)}, X_{13}^{(2)}\right) \cup \\
& \cup V\left(X_{11}^{(1)}, X_{11}^{(2)}, X_{22}^{(1)}, X_{22}^{(2)}-X_{12}^{(1)} X_{21}^{(1)}, X_{21}^{(2)}, X_{12}^{(2)}, X_{33}^{(1)}, X_{33}^{(2)}-X_{23}^{(1)} X_{32}^{(1)}-X_{13}^{(1)} X_{31}^{(1)}\right. \text {, } \\
& \left.X_{32}^{(2)}-X_{12}^{(1)} X_{31}^{(1)}, X_{23}^{(2)}-X_{13}^{(1)} X_{21}^{(1)}, X_{31}^{(2)}, X_{13}^{(2)}\right) \cup \\
& \cup V\left(X_{11}^{(1)}, X_{11}^{(2)}, X_{22}^{(1)}, X_{22}^{(2)}-X_{12}^{(1)} X_{21}^{(1)}, X_{21}^{(2)}, X_{12}^{(2)}, X_{33}^{(1)}, X_{33}^{(2)}-X_{13}^{(1)} X_{31}^{(1)}, X_{32}^{(1)}, X_{32}^{(2)}-X_{12}^{(1)} X_{31}^{(1)}, X_{31}^{(2)}, X_{13}^{(2)}\right) \cup \\
& \cup V\left(X_{11}^{(1)}, X_{11}^{(2)}, X_{22}^{(1)}, X_{22}^{(2)}-X_{12}^{(1)} X_{21}^{(1)}, X_{21}^{(2)}, X_{12}^{(2)}, X_{33}^{(1)}, X_{33}^{(2)}-X_{23}^{(1)} X_{32}^{(1)}-X_{13}^{(1)} X_{31}^{(1)}\right. \text {, } \\
& \left.X_{23}^{(2)}-X_{13}^{(1)} X_{21}^{(1)}, X_{32}^{(2)}-X_{12}^{(1)} X_{31}^{(1)}, X_{31}^{(2)}, X_{13}^{(2)}\right) \\
& =V\left(X_{11}^{(1)}, X_{11}^{(2)}, X_{22}^{(1)}, X_{22}^{(2)}-X_{12}^{(1)} X_{21}^{(1)}, X_{21}^{(2)}, X_{12}^{(2)}, X_{33}^{(1)}, X_{33}^{(2)}-X_{13}^{(1)} X_{31}^{(1)}, X_{23}^{(1)}, X_{23}^{(2)}-X_{13}^{(1)} X_{21}^{(1)}, X_{31}^{(2)}, X_{13}^{(2)}\right) \cup \\
& \cup V\left(X_{11}^{(1)}, X_{11}^{(2)}, X_{22}^{(1)}, X_{22}^{(2)}-X_{12}^{(1)} X_{21}^{(1)}, X_{21}^{(2)}, X_{12}^{(2)}, X_{33}^{(1)}, X_{33}^{(2)}-X_{23}^{(1)} X_{32}^{(1)}-X_{13}^{(1)} X_{31}^{(1)}\right. \text {, } \\
& \left.X_{32}^{(2)}-X_{12}^{(1)} X_{31}^{(1)}, X_{23}^{(2)}-X_{13}^{(1)} X_{21}^{(1)}, X_{31}^{(2)}, X_{13}^{(2)}\right) \cup \\
& \cup V\left(X_{11}^{(1)}, X_{11}^{(2)}, X_{22}^{(1)}, X_{22}^{(2)}-X_{12}^{(1)} X_{21}^{(1)}, X_{21}^{(2)}, X_{12}^{(2)}, X_{33}^{(1)}, X_{33}^{(2)}-X_{13}^{(1)} X_{31}^{(1)}, X_{32}^{(1)}, X_{32}^{(2)}-X_{12}^{(1)} X_{31}^{(1)}, X_{31}^{(2)}, X_{13}^{(2)}\right) \text {. }
\end{aligned}
$$

\section{Denotando}

$$
\begin{aligned}
C_{10}:= & V\left(X_{11}^{(1)}, X_{11}^{(2)}, X_{22}^{(1)}, X_{22}^{(2)}-X_{12}^{(1)} X_{21}^{(1)}, X_{21}^{(2)}, X_{12}^{(2)}, X_{33}^{(1)}, X_{33}^{(2)}-X_{13}^{(1)} X_{31}^{(1)}, X_{23}^{(1)}, X_{23}^{(2)}-X_{13}^{(1)} X_{21}^{(1)}, X_{31}^{(2)}, X_{13}^{(2)}\right), \\
C_{11}:= & V\left(X_{11}^{(1)}, X_{11}^{(2)}, X_{22}^{(1)}, X_{22}^{(2)}-X_{12}^{(1)} X_{21}^{(1)}, X_{21}^{(2)}, X_{12}^{(2)}, X_{33}^{(1)}, X_{33}^{(2)}-X_{23}^{(1)} X_{32}^{(1)}-X_{13}^{(1)} X_{31}^{(1)},\right. \\
& \left.X_{32}^{(2)}-X_{12}^{(1)} X_{31}^{(1)}, X_{23}^{(2)}-X_{13}^{(1)} X_{21}^{(1)}, X_{31}^{(2)}, X_{13}^{(2)}\right), \\
C_{12}:= & V\left(X_{11}^{(1)}, X_{11}^{(2)}, X_{22}^{(1)}, X_{22}^{(2)}-X_{12}^{(1)} X_{21}^{(1)}, X_{21}^{(2)}, X_{12}^{(2)}, X_{33}^{(1)}, X_{33}^{(2)}-X_{13}^{(1)} X_{31}^{(1)}, X_{32}^{(1)}, X_{32}^{(2)}-X_{12}^{(1)} X_{31}^{(1)}, X_{31}^{(2)}, X_{13}^{(2)}\right),
\end{aligned}
$$


tem-se

$$
W_{131}=C_{10} \cup C_{11} \cup C_{12} .
$$

Por outro lado, os polinômios que determinam

$$
W_{132}=V\left(X_{11}^{(1)}, X_{11}^{(2)}, X_{22}^{(1)}, p_{22}^{\mathbf{x}}, X_{21}^{(2)}, X_{12}^{(2)}, X_{33}^{(1)}, p_{32}^{\mathbf{x}}, p_{33}^{\mathbf{x}}, p_{34}^{\mathbf{x}}, \frac{p_{35}^{\mathbf{x}}}{X_{31}^{(2)}}, X_{13}^{(2)}\right) \subset k^{18}
$$

são

$$
\begin{aligned}
\mathbf{X} & =\left\{X_{11}^{(1)}, X_{11}^{(2)}, X_{22}^{(1)}, X_{21}^{(2)}, X_{12}^{(2)}, X_{33}^{(1)}, X_{13}^{(2)}\right\}, \\
p_{22}^{\mathbf{X}} & =X_{22}^{(2)}-X_{12}^{(1)} X_{21}^{(1)}, \\
p_{32}^{\mathbf{X}} & =X_{33}^{(2)}-X_{23}^{(1)} X_{32}^{(1)}-X_{13}^{(1)} X_{31}^{(1)}, \\
p_{33}^{\mathbf{X}} & =X_{12}^{(1)} X_{23}^{(1)} X_{31}^{(1)}+X_{13}^{(1)} X_{21}^{(1)} X_{32}^{(1)}-X_{13}^{(1)} X_{31}^{(2)}-X_{23}^{(1)} X_{32}^{(2)}-X_{23}^{(2)} X_{32}^{(1)}, \\
p_{34}^{\mathbf{X}} & =-X_{23}^{(2)} X_{32}^{(2)}+X_{12}^{(1)} X_{23}^{(1)} X_{31}^{(2)}+X_{13}^{(1)} X_{21}^{(1)} X_{32}^{(2)}-X_{13}^{(1)} X_{22}^{(2)} X_{31}^{(1)}+X_{12}^{(1)} X_{23}^{(2)} X_{31}^{(1)} \\
& =-X_{23}^{(2)} X_{32}^{(2)}+X_{12}^{(1)} X_{23}^{(1)} X_{31}^{(2)}+X_{13}^{(1)} X_{21}^{(1)} X_{32}^{(2)}+\underbrace{\left(-X_{13}^{(1)} X_{22}^{(2)}+X_{12}^{(1)} X_{23}^{(2)}\right)}_{=0} X_{31}^{(1)} \\
& =-X_{23}^{(2)} X_{32}^{(2)}+X_{12}^{(1)} X_{23}^{(1)} X_{31}^{(2)}+X_{13}^{(1)} X_{21}^{(1)} X_{32}^{(2)} \\
& =-\left(X_{23}^{(2)}-X_{13}^{(1)} X_{21}^{(1)}\right) X_{32}^{(2)}+X_{12}^{(1)} X_{23}^{(1)} X_{31}^{(2)}, \\
p_{35}^{\mathbf{X}} & =X_{12}^{(1)} X_{23}^{(2)}-X_{13}^{(1)} X_{22}^{(2)} \\
X_{31}^{(2)} & \\
& =X_{12}^{(1)} X_{23}^{(2)}-X_{13}^{(1)} X_{12}^{(1)} X_{21}^{(1)} \\
& =X_{12}^{(1)}\left(X_{23}^{(2)}-X_{13}^{(1)} X_{21}^{(1)}\right),
\end{aligned}
$$

obtendo a decomposição

$$
\begin{array}{rl}
W_{132}= & V\left(X_{11}^{(1)}, X_{11}^{(2)}, X_{22}^{(1)}, p_{22}^{\mathbf{x}}, X_{21}^{(2)}, X_{12}^{(2)}, X_{33}^{(1)}, p_{32}^{\mathbf{x}}, p_{33}^{\mathbf{x}}, p_{34}^{\mathbf{x}}, \frac{p_{35}^{\mathbf{x}}}{X_{31}^{(2)}}, X_{13}^{(2)}\right) \\
= & V\left(X_{11}^{(1)}, X_{11}^{(2)}, X_{22}^{(1)}, X_{22}^{(2)}-X_{12}^{(1)} X_{21}^{(1)}, X_{21}^{(2)}, X_{12}^{(2)}, X_{33}^{(1)}, X_{33}^{(2)}-X_{23}^{(1)} X_{32}^{(1)}-X_{13}^{(1)} X_{31}^{(1)},\right. \\
& X_{12}^{(1)} X_{23}^{(1)} X_{31}^{(1)}+X_{13}^{(1)} X_{21}^{(1)} X_{32}^{(1)}-X_{13}^{(1)} X_{31}^{(2)}-X_{23}^{(1)} X_{32}^{(2)}-X_{23}^{(2)} X_{32}^{(1)}, \\
& \left.-\left(X_{23}^{(2)}-X_{13}^{(1)} X_{21}^{(1)}\right) X_{32}^{(2)}+X_{12}^{(1)} X_{23}^{(1)} X_{31}^{(2)}, X_{12}^{(1)}\left(X_{23}^{(2)}-X_{13}^{(1)} X_{21}^{(1)}\right), X_{13}^{(2)}\right) \\
=V & V\left(X_{11}^{(1)}, X_{11}^{(2)}, X_{22}^{(1)}, X_{22}^{(2)}, X_{21}^{(2)}, X_{12}^{(2)}, X_{33}^{(1)}, X_{33}^{(2)}-X_{23}^{(1)} X_{32}^{(1)}-X_{13}^{(1)} X_{31}^{(1)},\right. \\
& \left.-X_{23}^{(1)} X_{32}^{(2)}-X_{23}^{(2)} X_{32}^{(1)}+X_{13}^{(1)} X_{21}^{(1)} X_{32}^{(1)}-X_{13}^{(1)} X_{31}^{(2)},\left(X_{23}^{(2)}-X_{13}^{(1)} X_{21}^{(1)}\right) X_{32}^{(2)}, X_{12}^{(1)}, X_{13}^{(2)}\right) \cup \\
\cup & V\left(X_{11}^{(1)}, X_{11}^{(2)}, X_{22}^{(1)}, X_{22}^{(2)}-X_{12}^{(1)} X_{21}^{(1)}, X_{21}^{(2)}, X_{12}^{(2)}, X_{33}^{(1)}, X_{33}^{(2)}-X_{23}^{(1)} X_{32}^{(1)}-X_{13}^{(1)} X_{31}^{(1)},\right. \\
& \left.-X_{23}^{(1)} X_{32}^{(2)}+X_{12}^{(1)} X_{23}^{(1)} X_{31}^{(1)}-X_{13}^{(1)} X_{31}^{(2)}, X_{12}^{(1)} X_{23}^{(1)} X_{31}^{(2)}, X_{23}^{(2)}-X_{13}^{(1)} X_{21}^{(1)}, X_{13}^{(2)}\right) \\
=V & V\left(X_{11}^{(1)}, X_{11}^{(2)}, X_{22}^{(1)}, X_{22}^{(2)}, X_{21}^{(2)}, X_{12}^{(2)}, X_{33}^{(1)}, X_{33}^{(2)}-X_{23}^{(1)} X_{32}^{(1)}-X_{13}^{(1)} X_{31}^{(1)},\right. \\
& \left.-X_{23}^{(2)} X_{32}^{(1)}+X_{13}^{(1)} X_{21}^{(1)} X_{32}^{(1)}-X_{13}^{(1)} X_{31}^{(2)}, X_{32}^{(2)}, X_{12}^{(1)}, X_{13}^{(2)}\right) \cup
\end{array}
$$




$$
\begin{aligned}
& \cup V\left(X_{11}^{(1)}, X_{11}^{(2)}, X_{22}^{(1)}, X_{22}^{(2)}, X_{21}^{(2)}, X_{12}^{(2)}, X_{33}^{(1)}, X_{33}^{(2)}-X_{23}^{(1)} X_{32}^{(1)}-X_{13}^{(1)} X_{31}^{(1)},\right. \\
& \left.X_{23}^{(1)} X_{32}^{(2)}+X_{13}^{(1)} X_{31}^{(2)}, X_{23}^{(2)}-X_{13}^{(1)} X_{21}^{(1)}, X_{12}^{(1)}, X_{13}^{(2)}\right) \cup \\
& \cup V\left(X_{11}^{(1)}, X_{11}^{(2)}, X_{22}^{(1)}, X_{22}^{(2)}, X_{21}^{(2)}, X_{12}^{(2)}, X_{33}^{(1)}, X_{33}^{(2)}-X_{23}^{(1)} X_{32}^{(1)}-X_{13}^{(1)} X_{31}^{(1)}\right. \text {, } \\
& \left.X_{23}^{(1)} X_{32}^{(2)}+X_{13}^{(1)} X_{31}^{(2)}, X_{12}^{(1)}, X_{23}^{(2)}-X_{13}^{(1)} X_{21}^{(1)}, X_{13}^{(2)}\right) \cup \\
& \cup V\left(X_{11}^{(1)}, X_{11}^{(2)}, X_{22}^{(1)}, X_{22}^{(2)}-X_{12}^{(1)} X_{21}^{(1)}, X_{21}^{(2)}, X_{12}^{(2)}, X_{33}^{(1)}, X_{33}^{(2)}-X_{13}^{(1)} X_{31}^{(1)}, X_{13}^{(1)} X_{31}^{(2)}\right. \text {, } \\
& \left.X_{23}^{(1)}, X_{23}^{(2)}-X_{13}^{(1)} X_{21}^{(1)}, X_{13}^{(2)}\right) \cup \\
& \cup V\left(X_{11}^{(1)}, X_{11}^{(2)}, X_{22}^{(1)}, X_{22}^{(2)}-X_{12}^{(1)} X_{21}^{(1)}, X_{21}^{(2)}, X_{12}^{(2)}, X_{33}^{(1)}, X_{33}^{(2)}-X_{23}^{(1)} X_{32}^{(1)}-X_{13}^{(1)} X_{31}^{(1)}\right. \text {, } \\
& \left.-X_{23}^{(1)} X_{32}^{(2)}+X_{12}^{(1)} X_{23}^{(1)} X_{31}^{(1)}, X_{31}^{(2)}, X_{23}^{(2)}-X_{13}^{(1)} X_{21}^{(1)}, X_{13}^{(2)}\right) \\
& =V\left(X_{11}^{(1)}, X_{11}^{(2)}, X_{22}^{(1)}, X_{22}^{(2)}, X_{21}^{(2)}, X_{12}^{(2)}, X_{33}^{(1)}, X_{33}^{(2)}-X_{23}^{(1)} X_{32}^{(1)}-X_{13}^{(1)} X_{31}^{(1)}\right. \text {, } \\
& \left.-X_{23}^{(2)} X_{32}^{(1)}+X_{13}^{(1)} X_{21}^{(1)} X_{32}^{(1)}-X_{13}^{(1)} X_{31}^{(2)}, X_{32}^{(2)}, X_{12}^{(1)}, X_{13}^{(2)}\right) \cup \\
& \cup V\left(X_{11}^{(1)}, X_{11}^{(2)}, X_{22}^{(1)}, X_{22}^{(2)}, X_{21}^{(2)}, X_{12}^{(2)}, X_{33}^{(1)}, X_{33}^{(2)}-X_{23}^{(1)} X_{32}^{(1)}-X_{13}^{(1)} X_{31}^{(1)}\right. \text {, } \\
& \left.X_{23}^{(1)} X_{32}^{(2)}+X_{13}^{(1)} X_{31}^{(2)}, X_{23}^{(2)}-X_{13}^{(1)} X_{21}^{(1)}, X_{12}^{(1)}, X_{13}^{(2)}\right) \cup \\
& \cup V\left(X_{11}^{(1)}, X_{11}^{(2)}, X_{22}^{(1)}, X_{22}^{(2)}, X_{21}^{(2)}, X_{12}^{(2)}, X_{33}^{(1)}, X_{33}^{(2)}-X_{23}^{(1)} X_{32}^{(1)}-X_{13}^{(1)} X_{31}^{(1)}\right. \text {, } \\
& \left.X_{23}^{(1)} X_{32}^{(2)}+X_{13}^{(1)} X_{31}^{(2)}, X_{12}^{(1)}, X_{23}^{(2)}-X_{13}^{(1)} X_{21}^{(1)}, X_{13}^{(2)}\right) \cup \\
& \cup V\left(X_{11}^{(1)}, X_{11}^{(2)}, X_{22}^{(1)}, X_{22}^{(2)}-X_{12}^{(1)} X_{21}^{(1)}, X_{21}^{(2)}, X_{12}^{(2)}, X_{33}^{(1)}, X_{33}^{(2)}, X_{13}^{(1)}, X_{23}^{(1)}, X_{23}^{(2)}, X_{13}^{(2)}\right) \cup \\
& \cup V\left(X_{11}^{(1)}, X_{11}^{(2)}, X_{22}^{(1)}, X_{22}^{(2)}-X_{12}^{(1)} X_{21}^{(1)}, X_{21}^{(2)}, X_{12}^{(2)}, X_{33}^{(1)}, X_{33}^{(2)}-X_{13}^{(1)} X_{31}^{(1)}, X_{31}^{(2)}\right. \text {, } \\
& \left.X_{23}^{(1)}, X_{23}^{(2)}-X_{13}^{(1)} X_{21}^{(1)}, X_{13}^{(2)}\right) \cup \\
& \cup V\left(X_{11}^{(1)}, X_{11}^{(2)}, X_{22}^{(1)}, X_{22}^{(2)}-X_{12}^{(1)} X_{21}^{(1)}, X_{21}^{(2)}, X_{12}^{(2)}, X_{33}^{(1)}, X_{33}^{(2)}-X_{13}^{(1)} X_{31}^{(1)}, X_{23}^{(1)}, X_{31}^{(2)}, X_{23}^{(2)}-X_{13}^{(1)} X_{21}^{(1)}, X_{13}^{(2)}\right) \cup \\
& \cup V\left(X_{11}^{(1)}, X_{11}^{(2)}, X_{22}^{(1)}, X_{22}^{(2)}-X_{12}^{(1)} X_{21}^{(1)}, X_{21}^{(2)}, X_{12}^{(2)}, X_{33}^{(1)}, X_{33}^{(2)}-X_{23}^{(1)} X_{32}^{(1)}-X_{13}^{(1)} X_{31}^{(1)}\right. \text {, } \\
& \left.X_{32}^{(2)}-X_{12}^{(1)} X_{31}^{(1)}, X_{31}^{(2)}, X_{23}^{(2)}-X_{13}^{(1)} X_{21}^{(1)}, X_{13}^{(2)}\right) \\
& =V\left(X_{11}^{(1)}, X_{11}^{(2)}, X_{22}^{(1)}, X_{22}^{(2)}, X_{21}^{(2)}, X_{12}^{(2)}, X_{33}^{(1)}, X_{33}^{(2)}-X_{23}^{(1)} X_{32}^{(1)}-X_{13}^{(1)} X_{31}^{(1)}\right. \text {, } \\
& \left.-X_{23}^{(2)} X_{32}^{(1)}+X_{13}^{(1)} X_{21}^{(1)} X_{32}^{(1)}-X_{13}^{(1)} X_{31}^{(2)}, X_{32}^{(2)}, X_{12}^{(1)}, X_{13}^{(2)}\right) \cup \\
& \cup V\left(X_{11}^{(1)}, X_{11}^{(2)}, X_{22}^{(1)}, X_{22}^{(2)}, X_{21}^{(2)}, X_{12}^{(2)}, X_{33}^{(1)}, X_{33}^{(2)}-X_{23}^{(1)} X_{32}^{(1)}-X_{13}^{(1)} X_{31}^{(1)}\right. \text {, } \\
& \left.X_{23}^{(1)} X_{32}^{(2)}+X_{13}^{(1)} X_{31}^{(2)}, X_{23}^{(2)}-X_{13}^{(1)} X_{21}^{(1)}, X_{12}^{(1)}, X_{13}^{(2)}\right) \cup \\
& \cup V\left(X_{11}^{(1)}, X_{11}^{(2)}, X_{22}^{(1)}, X_{22}^{(2)}-X_{12}^{(1)} X_{21}^{(1)}, X_{21}^{(2)}, X_{12}^{(2)}, X_{33}^{(1)}, X_{33}^{(2)}, X_{13}^{(1)}, X_{23}^{(1)}, X_{23}^{(2)}, X_{13}^{(2)}\right) \cup \\
& \cup V\left(X_{11}^{(1)}, X_{11}^{(2)}, X_{22}^{(1)}, X_{22}^{(2)}-X_{12}^{(1)} X_{21}^{(1)}, X_{21}^{(2)}, X_{12}^{(2)}, X_{33}^{(1)}, X_{33}^{(2)}-X_{13}^{(1)} X_{31}^{(1)}, X_{31}^{(2)}, X_{23}^{(1)}, X_{23}^{(2)}-X_{13}^{(1)} X_{21}^{(1)}, X_{13}^{(2)}\right) \cup
\end{aligned}
$$




$$
\begin{aligned}
& \cup V\left(X_{11}^{(1)}, X_{11}^{(2)}, X_{22}^{(1)}, X_{22}^{(2)}-X_{12}^{(1)} X_{21}^{(1)}, X_{21}^{(2)}, X_{12}^{(2)}, X_{33}^{(1)}, X_{33}^{(2)}-X_{23}^{(1)} X_{32}^{(1)}-X_{13}^{(1)} X_{31}^{(1)},\right. \\
& \left.\quad X_{32}^{(2)}-X_{12}^{(1)} X_{31}^{(1)}, X_{31}^{(2)}, X_{23}^{(2)}-X_{13}^{(1)} X_{21}^{(1)}, X_{13}^{(2)}\right) .
\end{aligned}
$$

\section{Como}

$$
\begin{aligned}
& C_{4}= V\left(X_{11}^{(1)}, X_{11}^{(2)}, X_{22}^{(1)}, X_{22}^{(2)}, X_{21}^{(2)}, X_{12}^{(2)}, X_{33}^{(1)}, X_{33}^{(2)}-X_{23}^{(1)} X_{32}^{(1)}-X_{13}^{(1)} X_{31}^{(1)},\right. \\
&\left.-X_{23}^{(2)} X_{32}^{(1)}+X_{13}^{(1)} X_{21}^{(1)} X_{32}^{(1)}-X_{13}^{(1)} X_{31}^{(2)}, X_{32}^{(2)}, X_{12}^{(1)}, X_{13}^{(2)}\right), \\
& C_{5}=V\left(X_{11}^{(1)}, X_{11}^{(2)}, X_{22}^{(1)}, X_{22}^{(2)}, X_{21}^{(2)}, X_{12}^{(2)}, X_{33}^{(1)}, X_{33}^{(2)}-X_{23}^{(1)} X_{32}^{(1)}-X_{13}^{(1)} X_{31}^{(1)},\right. \\
&\left.X_{23}^{(1)} X_{32}^{(2)}+X_{13}^{(1)} X_{31}^{(2)}, X_{23}^{(2)}-X_{13}^{(1)} X_{21}^{(1)}, X_{12}^{(1)}, X_{13}^{(2)}\right), \\
& C_{10}=V\left(X_{11}^{(1)}, X_{11}^{(2)}, X_{22}^{(1)}, X_{22}^{(2)}-X_{12}^{(1)} X_{21}^{(1)}, X_{21}^{(2)}, X_{12}^{(2)}, X_{33}^{(1)}, X_{33}^{(2)}-X_{13}^{(1)} X_{31}^{(1)}, X_{31}^{(2)}, X_{23}^{(1)}, X_{23}^{(2)}-X_{13}^{(1)} X_{21}^{(1)}, X_{13}^{(2)}\right), \\
& C_{11}=V\left(X_{11}^{(1)}, X_{11}^{(2)}, X_{22}^{(1)}, X_{22}^{(2)}-X_{12}^{(1)} X_{21}^{(1)}, X_{21}^{(2)}, X_{12}^{(2)}, X_{33}^{(1)}, X_{33}^{(2)}-X_{23}^{(1)} X_{32}^{(1)}-X_{13}^{(1)} X_{31}^{(1)},\right. \\
&\left.X_{32}^{(2)}-X_{12}^{(1)} X_{31}^{(1)}, X_{31}^{(2)}, X_{23}^{(2)}-X_{13}^{(1)} X_{21}^{(1)}, X_{13}^{(2)}\right)
\end{aligned}
$$

e denotando

$$
C_{13}:=V\left(X_{11}^{(1)}, X_{11}^{(2)}, X_{22}^{(1)}, X_{22}^{(2)}-X_{12}^{(1)} X_{21}^{(1)}, X_{21}^{(2)}, X_{12}^{(2)}, X_{33}^{(1)}, X_{33}^{(2)}, X_{13}^{(1)}, X_{23}^{(1)}, X_{23}^{(2)}, X_{13}^{(2)}\right),
$$

temos

$$
W_{132}=C_{4} \cup C_{5} \cup C_{10} \cup C_{11} \cup C_{13}
$$

e das equaçõess (B.8) e (B.9)

$$
W_{13}=C_{4} \cup C_{5} \cup C_{10} \cup C_{11} \cup C_{12} \cup C_{13} .
$$

\section{B.2.4 Decomposição de $W_{14}$}

Similarmente aos casos anteriores, temos que

$$
W_{14}=V\left(X_{11}^{(1)}, X_{11}^{(2)}, X_{22}^{(1)}, p_{22}^{\mathbf{x}}, X_{21}^{(2)}, X_{12}^{(2)}, X_{33}^{(1)}, p_{32}^{\mathbf{x}}, p_{33}^{\mathbf{x}}, p_{34}^{\mathbf{x}}, p_{35}^{\mathbf{x}}, \frac{p_{36}^{\mathbf{x}}}{X_{13}^{(2)}}\right) \subset k^{18},
$$

com

$$
\begin{aligned}
\mathbf{X}= & \left\{X_{11}^{(1)}, X_{11}^{(2)}, X_{22}^{(1)}, X_{21}^{(2)}, X_{12}^{(2)}, X_{33}^{(1)}\right\} \\
p_{22}^{\mathbf{X}}= & X_{22}^{(2)}-X_{12}^{(1)} X_{21}^{(1)}, \\
p_{32}^{\mathbf{X}}= & X_{33}^{(2)}-X_{23}^{(1)} X_{32}^{(1)}-X_{13}^{(1)} X_{31}^{(1)}, \\
p_{33}^{\mathbf{X}}= & X_{12}^{(1)} X_{23}^{(1)} X_{31}^{(1)}+X_{13}^{(1)} X_{21}^{(1)} X_{32}^{(1)}-X_{13}^{(1)} X_{31}^{(2)}-X_{23}^{(1)} X_{32}^{(2)}-X_{13}^{(2)} X_{31}^{(1)}-X_{23}^{(2)} X_{32}^{(1)}, \\
p_{34}^{\mathbf{X}}= & -X_{23}^{(2)} X_{32}^{(2)}-X_{13}^{(2)} X_{31}^{(2)}+X_{12}^{(1)} X_{23}^{(1)} X_{31}^{(2)}+X_{13}^{(1)} X_{21}^{(1)} X_{32}^{(2)}-X_{13}^{(1)} X_{22}^{(2)} X_{31}^{(1)}+ \\
& +X_{12}^{(1)} X_{23}^{(2)} X_{31}^{(1)}+\underbrace{X_{13}^{(1)} X_{21}^{(2)} X_{32}^{(1)}}_{=0}+X_{13}^{(2)} X_{21}^{(1)} X_{32}^{(1)} \\
= & -X_{23}^{(2)} X_{32}^{(2)}-X_{13}^{(2)} X_{31}^{(2)}+X_{12}^{(1)} X_{23}^{(1)} X_{31}^{(2)}+X_{13}^{(1)} X_{21}^{(1)} X_{32}^{(2)}-X_{13}^{(1)} X_{22}^{(2)} X_{31}^{(1)}+X_{12}^{(1)} X_{23}^{(2)} X_{31}^{(1)}+X_{13}^{(2)} X_{21}^{(1)} X_{32}^{(1)},
\end{aligned}
$$




$$
\begin{aligned}
p_{35}^{\mathbf{x}} & =-X_{13}^{(1)} \underbrace{X_{22}^{(2)} X_{31}^{(2)}}_{=0}+X_{12}^{(1)} X_{23}^{(2)} X_{31}^{(2)}+\underbrace{X_{13}^{(1)} X_{21}^{(2)} X_{32}^{(2)}}_{=0}+X_{13}^{(2)} X_{21}^{(1)} X_{32}^{(2)}-X_{13}^{(2)} X_{22}^{(2)} X_{31}^{(1)}+\underbrace{X_{13}^{(2)} X_{21}^{(2)} X_{32}^{(1)}}_{=0} \\
& =X_{12}^{(1)} X_{23}^{(2)} X_{31}^{(2)}+X_{13}^{(2)} X_{21}^{(1)} X_{32}^{(2)}-X_{13}^{(2)} X_{22}^{(2)} X_{31}^{(1)}, \\
\frac{p_{36}^{\mathbf{X}}}{X_{13}^{(2)}} & =\underbrace{X_{21}^{(2)} X_{32}^{(2)}}_{=0}-X_{22}^{(2)} X_{31}^{(2)} \\
& =-X_{22}^{(2)} X_{31}^{(2)},
\end{aligned}
$$

segue da última equação que $W_{14}=W_{141} \cup W_{142}$, com

$$
\begin{aligned}
W_{141} & =V\left(X_{11}^{(1)}, X_{11}^{(2)}, X_{22}^{(1)}, p_{22}^{\mathbf{X}}, X_{21}^{(2)}, X_{12}^{(2)}, X_{33}^{(1)}, p_{32}^{\mathbf{X}}, p_{33}^{\mathbf{X}}, p_{34}^{\mathbf{X}}, p_{35}^{\mathbf{X}}, X_{22}^{(2)}\right) \subset k^{18} \\
\mathbf{X} & =\left\{X_{11}^{(1)}, X_{11}^{(2)}, X_{22}^{(1)}, X_{21}^{(2)}, X_{12}^{(2)}, X_{33}^{(1)}, X_{22}^{(2)}\right\} \\
\mathrm{e} & \\
W_{142} & =V\left(X_{11}^{(1)}, X_{11}^{(2)}, X_{22}^{(1)}, p_{22}^{\mathbf{X}}, X_{21}^{(2)}, X_{12}^{(2)}, X_{33}^{(1)}, p_{32}^{\mathbf{X}}, p_{33}^{\mathbf{X}}, p_{34}^{\mathbf{X}}, p_{35}^{\mathbf{X}}, X_{31}^{(2)}\right) \subset k^{18} \\
\mathbf{X} & =\left\{X_{11}^{(1)}, X_{11}^{(2)}, X_{22}^{(1)}, X_{21}^{(2)}, X_{12}^{(2)}, X_{33}^{(1)}, X_{31}^{(2)}\right\},
\end{aligned}
$$

Os polinômios que determinam

$$
W_{141}=V\left(X_{11}^{(1)}, X_{11}^{(2)}, X_{22}^{(1)}, p_{22}^{\mathbf{x}}, X_{21}^{(2)}, X_{12}^{(2)}, X_{33}^{(1)}, p_{32}^{\mathbf{x}}, p_{33}^{\mathbf{x}}, p_{34}^{\mathbf{x}}, p_{35}^{\mathbf{x}}, X_{22}^{(2)}\right) \subset k^{18}
$$

são

$$
\begin{aligned}
\mathbf{X} & =\left\{X_{11}^{(1)}, X_{11}^{(2)}, X_{22}^{(1)}, X_{21}^{(2)}, X_{12}^{(2)}, X_{33}^{(1)}, X_{22}^{(2)}\right\}, \\
p_{22}^{\mathbf{X}} & =\underbrace{X_{22}^{(2)}}_{=0}-X_{12}^{(1)} X_{21}^{(1)} \\
& =-X_{12}^{(1)} X_{21}^{(1)} \\
p_{32}^{\mathbf{X}} & =X_{33}^{(2)}-X_{23}^{(1)} X_{32}^{(1)}-X_{13}^{(1)} X_{31}^{(1)}, \\
p_{33}^{\mathbf{X}} & =X_{12}^{(1)} X_{23}^{(1)} X_{31}^{(1)}+X_{13}^{(1)} X_{21}^{(1)} X_{32}^{(1)}-X_{13}^{(1)} X_{31}^{(2)}-X_{23}^{(1)} X_{32}^{(2)}-X_{13}^{(2)} X_{31}^{(1)}-X_{23}^{(2)} X_{32}^{(1)}, \\
p_{34}^{\mathbf{X}} & =-X_{23}^{(2)} X_{32}^{(2)}-X_{13}^{(2)} X_{31}^{(2)}+X_{12}^{(1)} X_{23}^{(1)} X_{31}^{(2)}+X_{13}^{(1)} X_{21}^{(1)} X_{32}^{(2)}-\underbrace{X_{13}^{(1)} X_{22}^{(2)} X_{31}^{(1)}}_{=0}+X_{12}^{(1)} X_{23}^{(2)} X_{31}^{(1)}+X_{13}^{(2)} X_{21}^{(1)} X_{32}^{(1)} \\
& =-X_{23}^{(2)} X_{32}^{(2)}-X_{13}^{(2)} X_{31}^{(2)}+X_{12}^{(1)} X_{23}^{(1)} X_{31}^{(2)}+X_{13}^{(1)} X_{21}^{(1)} X_{32}^{(2)}+X_{12}^{(1)} X_{23}^{(2)} X_{31}^{(1)}+X_{13}^{(2)} X_{21}^{(1)} X_{32}^{(1)}, \\
p_{35}^{\mathbf{X}} & =X_{12}^{(1)} X_{23}^{(2)} X_{31}^{(2)}+X_{13}^{(2)} X_{21}^{(1)} X_{32}^{(2)}-\underbrace{X_{13}^{(2)} X_{22}^{(2)} X_{31}^{(1)}}_{=0} \\
& =X_{12}^{(1)} X_{23}^{(2)} X_{31}^{(2)}+X_{13}^{(2)} X_{21}^{(1)} X_{32}^{(2)}
\end{aligned}
$$

e portanto podemos obter a decomposição para $W_{141}$ da seguinte forma

$$
\begin{aligned}
W_{141}= & V\left(X_{11}^{(1)}, X_{11}^{(2)}, X_{22}^{(1)}, p_{22}^{\mathbf{X}}, X_{21}^{(2)}, X_{12}^{(2)}, X_{33}^{(1)}, p_{32}^{\mathbf{x}}, p_{33}^{\mathbf{x}}, p_{34}^{\mathbf{x}}, p_{35}^{\mathbf{X}}, X_{22}^{(2)}\right) \\
= & V\left(X_{11}^{(1)}, X_{11}^{(2)}, X_{22}^{(1)}, X_{12}^{(1)} X_{21}^{(1)}, X_{21}^{(2)}, X_{12}^{(2)}, X_{33}^{(1)}, X_{33}^{(2)}-X_{23}^{(1)} X_{32}^{(1)}-X_{13}^{(1)} X_{31}^{(1)},\right. \\
& -X_{23}^{(1)} X_{32}^{(2)}-X_{23}^{(2)} X_{32}^{(1)}+X_{12}^{(1)} X_{23}^{(1)} X_{31}^{(1)}+X_{13}^{(1)} X_{21}^{(1)} X_{32}^{(1)}-X_{13}^{(1)} X_{31}^{(2)}-X_{13}^{(2)} X_{31}^{(1)}, \\
& -X_{23}^{(2)} X_{32}^{(2)}+X_{12}^{(1)} X_{23}^{(1)} X_{31}^{(2)}+X_{12}^{(1)} X_{23}^{(2)} X_{31}^{(1)}+X_{13}^{(1)} X_{21}^{(1)} X_{32}^{(2)}+X_{13}^{(2)} X_{21}^{(1)} X_{32}^{(1)}-X_{13}^{(2)} X_{31}^{(2)}, \\
& \left.X_{12}^{(1)} X_{23}^{(2)} X_{31}^{(2)}+X_{13}^{(2)} X_{21}^{(1)} X_{32}^{(2)}, X_{22}^{(2)}\right)
\end{aligned}
$$




$$
\begin{aligned}
& =V\left(X_{11}^{(1)}, X_{11}^{(2)}, X_{22}^{(1)}, X_{12}^{(1)}, X_{21}^{(2)}, X_{12}^{(2)}, X_{33}^{(1)}, X_{33}^{(2)}-X_{23}^{(1)} X_{32}^{(1)}-X_{13}^{(1)} X_{31}^{(1)}\right. \text {, } \\
& -X_{23}^{(1)} X_{32}^{(2)}-X_{23}^{(2)} X_{32}^{(1)}+X_{13}^{(1)} X_{21}^{(1)} X_{32}^{(1)}-X_{13}^{(1)} X_{31}^{(2)}-X_{13}^{(2)} X_{31}^{(1)} \\
& \left.-X_{23}^{(2)} X_{32}^{(2)}+X_{13}^{(1)} X_{21}^{(1)} X_{32}^{(2)}+X_{13}^{(2)} X_{21}^{(1)} X_{32}^{(1)}-X_{13}^{(2)} X_{31}^{(2)}, X_{13}^{(2)} X_{21}^{(1)} X_{32}^{(2)}, X_{22}^{(2)}\right) \cup \\
& \cup V\left(X_{11}^{(1)}, X_{11}^{(2)}, X_{22}^{(1)}, X_{21}^{(1)}, X_{21}^{(2)}, X_{12}^{(2)}, X_{33}^{(1)}, X_{33}^{(2)}-X_{23}^{(1)} X_{32}^{(1)}-X_{13}^{(1)} X_{31}^{(1)}\right. \text {, } \\
& -X_{23}^{(1)} X_{32}^{(2)}-X_{23}^{(2)} X_{32}^{(1)}+X_{12}^{(1)} X_{23}^{(1)} X_{31}^{(1)}-X_{13}^{(1)} X_{31}^{(2)}-X_{13}^{(2)} X_{31}^{(1)} \\
& \left.-X_{23}^{(2)} X_{32}^{(2)}+X_{12}^{(1)} X_{23}^{(1)} X_{31}^{(2)}+X_{12}^{(1)} X_{23}^{(2)} X_{31}^{(1)}-X_{13}^{(2)} X_{31}^{(2)}, X_{12}^{(1)} X_{23}^{(2)} X_{31}^{(2)}, X_{22}^{(2)}\right) \\
& =V\left(X_{11}^{(1)}, X_{11}^{(2)}, X_{22}^{(1)}, X_{12}^{(1)}, X_{21}^{(2)}, X_{12}^{(2)}, X_{33}^{(1)}, X_{33}^{(2)}-X_{23}^{(1)} X_{32}^{(1)}-X_{13}^{(1)} X_{31}^{(1)}\right. \text {, } \\
& \left.-X_{23}^{(1)} X_{32}^{(2)}-X_{23}^{(2)} X_{32}^{(1)}+X_{13}^{(1)} X_{21}^{(1)} X_{32}^{(1)}-X_{13}^{(1)} X_{31}^{(2)}, X_{23}^{(2)} X_{32}^{(2)}-X_{13}^{(1)} X_{21}^{(1)} X_{32}^{(2)}, X_{13}^{(2)}, X_{22}^{(2)}\right) \cup \\
& \cup V\left(X_{11}^{(1)}, X_{11}^{(2)}, X_{22}^{(1)}, X_{12}^{(1)}, X_{21}^{(2)}, X_{12}^{(2)}, X_{33}^{(1)}, X_{33}^{(2)}-X_{23}^{(1)} X_{32}^{(1)}-X_{13}^{(1)} X_{31}^{(1)}\right. \text {, } \\
& \left.X_{23}^{(1)} X_{32}^{(2)}+X_{23}^{(2)} X_{32}^{(1)}+X_{13}^{(1)} X_{31}^{(2)}+X_{13}^{(2)} X_{31}^{(1)}, X_{23}^{(2)} X_{32}^{(2)}+X_{13}^{(2)} X_{31}^{(2)}, X_{21}^{(1)}, X_{22}^{(2)}\right) \cup \\
& \cup V\left(X_{11}^{(1)}, X_{11}^{(2)}, X_{22}^{(1)}, X_{12}^{(1)}, X_{21}^{(2)}, X_{12}^{(2)}, X_{33}^{(1)}, X_{33}^{(2)}-X_{23}^{(1)} X_{32}^{(1)}-X_{13}^{(1)} X_{31}^{(1)}\right. \text {, } \\
& \left.X_{23}^{(2)} X_{32}^{(1)}-X_{13}^{(1)} X_{21}^{(1)} X_{32}^{(1)}+X_{13}^{(1)} X_{31}^{(2)}+X_{13}^{(2)} X_{31}^{(1)}, X_{13}^{(2)} X_{21}^{(1)} X_{32}^{(1)}-X_{13}^{(2)} X_{31}^{(2)}, X_{32}^{(2)}, X_{22}^{(2)}\right) \cup \\
& \cup V\left(X_{11}^{(1)}, X_{11}^{(2)}, X_{22}^{(1)}, X_{21}^{(1)}, X_{21}^{(2)}, X_{12}^{(2)}, X_{33}^{(1)}, X_{33}^{(2)}-X_{23}^{(1)} X_{32}^{(1)}-X_{13}^{(1)} X_{31}^{(1)}\right. \text {, } \\
& \left.X_{23}^{(1)} X_{32}^{(2)}+X_{23}^{(2)} X_{32}^{(1)}+X_{13}^{(1)} X_{31}^{(2)}+X_{13}^{(2)} X_{31}^{(1)}, X_{23}^{(2)} X_{32}^{(2)}+X_{13}^{(2)} X_{31}^{(2)}, X_{12}^{(1)}, X_{22}^{(2)}\right) \cup \\
& \cup V\left(X_{11}^{(1)}, X_{11}^{(2)}, X_{22}^{(1)}, X_{21}^{(1)}, X_{21}^{(2)}, X_{12}^{(2)}, X_{33}^{(1)}, X_{33}^{(2)}-X_{23}^{(1)} X_{32}^{(1)}-X_{13}^{(1)} X_{31}^{(1)}\right. \text {, } \\
& \left.X_{23}^{(1)} X_{32}^{(2)}-X_{12}^{(1)} X_{23}^{(1)} X_{31}^{(1)}+X_{13}^{(1)} X_{31}^{(2)}+X_{13}^{(2)} X_{31}^{(1)}, X_{12}^{(1)} X_{23}^{(1)} X_{31}^{(2)}-X_{13}^{(2)} X_{31}^{(2)}, X_{23}^{(2)}, X_{22}^{(2)}\right) \cup \\
& \cup V\left(X_{11}^{(1)}, X_{11}^{(2)}, X_{22}^{(1)}, X_{21}^{(1)}, X_{21}^{(2)}, X_{12}^{(2)}, X_{33}^{(1)}, X_{33}^{(2)}-X_{23}^{(1)} X_{32}^{(1)}-X_{13}^{(1)} X_{31}^{(1)}\right. \text {, } \\
& \left.X_{23}^{(1)} X_{32}^{(2)}+X_{23}^{(2)} X_{32}^{(1)}-X_{12}^{(1)} X_{23}^{(1)} X_{31}^{(1)}+X_{13}^{(2)} X_{31}^{(1)}, X_{23}^{(2)} X_{32}^{(2)}-X_{12}^{(1)} X_{23}^{(2)} X_{31}^{(1)}, X_{31}^{(2)}, X_{22}^{(2)}\right) \\
& =V\left(X_{11}^{(1)}, X_{11}^{(2)}, X_{22}^{(1)}, X_{12}^{(1)}, X_{21}^{(2)}, X_{12}^{(2)}, X_{33}^{(1)}, X_{33}^{(2)}-X_{23}^{(1)} X_{32}^{(1)}-X_{13}^{(1)} X_{31}^{(1)}\right. \text {, } \\
& \left.X_{23}^{(2)} X_{32}^{(1)}-X_{13}^{(1)} X_{21}^{(1)} X_{32}^{(1)}+X_{13}^{(1)} X_{31}^{(2)}, X_{32}^{(2)}, X_{13}^{(2)}, X_{22}^{(2)}\right) \cup \\
& \cup V\left(X_{11}^{(1)}, X_{11}^{(2)}, X_{22}^{(1)}, X_{12}^{(1)}, X_{21}^{(2)}, X_{12}^{(2)}, X_{33}^{(1)}, X_{33}^{(2)}-X_{23}^{(1)} X_{32}^{(1)}-X_{13}^{(1)} X_{31}^{(1)}\right. \text {, } \\
& \left.X_{23}^{(1)} X_{32}^{(2)}+X_{13}^{(1)} X_{31}^{(2)}, X_{23}^{(2)}-X_{13}^{(1)} X_{21}^{(1)}, X_{13}^{(2)}, X_{22}^{(2)}\right) \cup \\
& \cup V\left(X_{11}^{(1)}, X_{11}^{(2)}, X_{22}^{(1)}, X_{12}^{(1)}, X_{21}^{(2)}, X_{12}^{(2)}, X_{33}^{(1)}, X_{33}^{(2)}-X_{23}^{(1)} X_{32}^{(1)}-X_{13}^{(1)} X_{31}^{(1)}\right. \text {, } \\
& \left.X_{23}^{(1)} X_{32}^{(2)}+X_{23}^{(2)} X_{32}^{(1)}+X_{13}^{(1)} X_{31}^{(2)}+X_{13}^{(2)} X_{31}^{(1)}, X_{23}^{(2)} X_{32}^{(2)}+X_{13}^{(2)} X_{31}^{(2)}, X_{21}^{(1)}, X_{22}^{(2)}\right) \cup \\
& \cup V\left(X_{11}^{(1)}, X_{11}^{(2)}, X_{22}^{(1)}, X_{12}^{(1)}, X_{21}^{(2)}, X_{12}^{(2)}, X_{33}^{(1)}, X_{33}^{(2)}-X_{23}^{(1)} X_{32}^{(1)}-X_{13}^{(1)} X_{31}^{(1)}\right. \text {, } \\
& \left.X_{23}^{(2)} X_{32}^{(1)}-X_{13}^{(1)} X_{21}^{(1)} X_{32}^{(1)}+X_{13}^{(1)} X_{31}^{(2)}, X_{13}^{(2)}, X_{32}^{(2)}, X_{22}^{(2)}\right) \cup
\end{aligned}
$$




$$
\begin{aligned}
& \cup V\left(X_{11}^{(1)}, X_{11}^{(2)}, X_{22}^{(1)}, X_{12}^{(1)}, X_{21}^{(2)}, X_{12}^{(2)}, X_{33}^{(1)}, X_{33}^{(2)}-X_{23}^{(1)} X_{32}^{(1)}-X_{13}^{(1)} X_{31}^{(1)}\right. \text {, } \\
& \left.X_{23}^{(2)} X_{32}^{(1)}+X_{13}^{(2)} X_{31}^{(1)}, X_{21}^{(1)} X_{32}^{(1)}-X_{31}^{(2)}, X_{32}^{(2)}, X_{22}^{(2)}\right) \cup \\
& \cup V\left(X_{11}^{(1)}, X_{11}^{(2)}, X_{22}^{(1)}, X_{21}^{(1)}, X_{21}^{(2)}, X_{12}^{(2)}, X_{33}^{(1)}, X_{33}^{(2)}-X_{23}^{(1)} X_{32}^{(1)}-X_{13}^{(1)} X_{31}^{(1)}\right. \text {, } \\
& \left.X_{23}^{(1)} X_{32}^{(2)}+X_{23}^{(2)} X_{32}^{(1)}+X_{13}^{(1)} X_{31}^{(2)}+X_{13}^{(2)} X_{31}^{(1)}, X_{23}^{(2)} X_{32}^{(2)}+X_{13}^{(2)} X_{31}^{(2)}, X_{12}^{(1)}, X_{22}^{(2)}\right) \cup \\
& \cup V\left(X_{11}^{(1)}, X_{11}^{(2)}, X_{22}^{(1)}, X_{21}^{(1)}, X_{21}^{(2)}, X_{12}^{(2)}, X_{33}^{(1)}, X_{33}^{(2)}-X_{23}^{(1)} X_{32}^{(1)}-X_{13}^{(1)} X_{31}^{(1)}\right. \text {, } \\
& \left.X_{23}^{(1)} X_{32}^{(2)}-X_{12}^{(1)} X_{23}^{(1)} X_{31}^{(1)}+X_{13}^{(2)} X_{31}^{(1)}, X_{31}^{(2)}, X_{23}^{(2)}, X_{22}^{(2)}\right) \cup \\
& \cup V\left(X_{11}^{(1)}, X_{11}^{(2)}, X_{22}^{(1)}, X_{21}^{(1)}, X_{21}^{(2)}, X_{12}^{(2)}, X_{33}^{(1)}, X_{33}^{(2)}-X_{23}^{(1)} X_{32}^{(1)}-X_{13}^{(1)} X_{31}^{(1)}\right. \text {, } \\
& \left.X_{23}^{(1)} X_{32}^{(2)}+X_{13}^{(1)} X_{31}^{(2)}, X_{12}^{(1)} X_{23}^{(1)}-X_{13}^{(2)}, X_{23}^{(2)}, X_{22}^{(2)}\right) \cup \\
& \cup V\left(X_{11}^{(1)}, X_{11}^{(2)}, X_{22}^{(1)}, X_{21}^{(1)}, X_{21}^{(2)}, X_{12}^{(2)}, X_{33}^{(1)}, X_{33}^{(2)}-X_{23}^{(1)} X_{32}^{(1)}-X_{13}^{(1)} X_{31}^{(1)}\right. \text {, } \\
& \left.X_{23}^{(1)} X_{32}^{(2)}-X_{12}^{(1)} X_{23}^{(1)} X_{31}^{(1)}+X_{13}^{(2)} X_{31}^{(1)}, X_{23}^{(2)}, X_{31}^{(2)}, X_{22}^{(2)}\right) \cup \\
& \cup V\left(X_{11}^{(1)}, X_{11}^{(2)}, X_{22}^{(1)}, X_{21}^{(1)}, X_{21}^{(2)}, X_{12}^{(2)}, X_{33}^{(1)}, X_{33}^{(2)}-X_{23}^{(1)} X_{32}^{(1)}-X_{13}^{(1)} X_{31}^{(1)}\right. \text {, } \\
& \left.X_{23}^{(2)} X_{32}^{(1)}+X_{13}^{(2)} X_{31}^{(1)}, X_{32}^{(2)}-X_{12}^{(1)} X_{31}^{(1)}, X_{31}^{(2)}, X_{22}^{(2)}\right) \\
& =V\left(X_{11}^{(1)}, X_{11}^{(2)}, X_{22}^{(1)}, X_{12}^{(1)}, X_{21}^{(2)}, X_{12}^{(2)}, X_{33}^{(1)}, X_{33}^{(2)}-X_{23}^{(1)} X_{32}^{(1)}-X_{13}^{(1)} X_{31}^{(1)}\right. \text {, } \\
& \left.X_{23}^{(2)} X_{32}^{(1)}-X_{13}^{(1)} X_{21}^{(1)} X_{32}^{(1)}+X_{13}^{(1)} X_{31}^{(2)}, X_{32}^{(2)}, X_{13}^{(2)}, X_{22}^{(2)}\right) \cup \\
& \cup V\left(X_{11}^{(1)}, X_{11}^{(2)}, X_{22}^{(1)}, X_{12}^{(1)}, X_{21}^{(2)}, X_{12}^{(2)}, X_{33}^{(1)}, X_{33}^{(2)}-X_{23}^{(1)} X_{32}^{(1)}-X_{13}^{(1)} X_{31}^{(1)}\right. \text {, } \\
& \left.X_{23}^{(1)} X_{32}^{(2)}+X_{13}^{(1)} X_{31}^{(2)}, X_{23}^{(2)}-X_{13}^{(1)} X_{21}^{(1)}, X_{13}^{(2)}, X_{22}^{(2)}\right) \cup \\
& \cup V\left(X_{11}^{(1)}, X_{11}^{(2)}, X_{22}^{(1)}, X_{12}^{(1)}, X_{21}^{(2)}, X_{12}^{(2)}, X_{33}^{(1)}, X_{33}^{(2)}-X_{23}^{(1)} X_{32}^{(1)}-X_{13}^{(1)} X_{31}^{(1)}\right. \text {, } \\
& \left.X_{23}^{(1)} X_{32}^{(2)}+X_{23}^{(2)} X_{32}^{(1)}+X_{13}^{(1)} X_{31}^{(2)}+X_{13}^{(2)} X_{31}^{(1)}, X_{23}^{(2)} X_{32}^{(2)}+X_{13}^{(2)} X_{31}^{(2)}, X_{21}^{(1)}, X_{22}^{(2)}\right) \cup \\
& \cup V\left(X_{11}^{(1)}, X_{11}^{(2)}, X_{22}^{(1)}, X_{12}^{(1)}, X_{21}^{(2)}, X_{12}^{(2)}, X_{33}^{(1)}, X_{33}^{(2)}-X_{23}^{(1)} X_{32}^{(1)}-X_{13}^{(1)} X_{31}^{(1)}\right. \text {, } \\
& \left.X_{23}^{(2)} X_{32}^{(1)}+X_{13}^{(2)} X_{31}^{(1)}, X_{21}^{(1)} X_{32}^{(1)}-X_{31}^{(2)}, X_{32}^{(2)}, X_{22}^{(2)}\right) \cup \\
& \cup V\left(X_{11}^{(1)}, X_{11}^{(2)}, X_{22}^{(1)}, X_{21}^{(1)}, X_{21}^{(2)}, X_{12}^{(2)}, X_{33}^{(1)}, X_{33}^{(2)}-X_{23}^{(1)} X_{32}^{(1)}-X_{13}^{(1)} X_{31}^{(1)}\right. \text {, } \\
& \left.X_{23}^{(1)} X_{32}^{(2)}-X_{12}^{(1)} X_{23}^{(1)} X_{31}^{(1)}+X_{13}^{(2)} X_{31}^{(1)}, X_{31}^{(2)}, X_{23}^{(2)}, X_{22}^{(2)}\right) \cup \\
& \cup V\left(X_{11}^{(1)}, X_{11}^{(2)}, X_{22}^{(1)}, X_{21}^{(1)}, X_{21}^{(2)}, X_{12}^{(2)}, X_{33}^{(1)}, X_{33}^{(2)}-X_{23}^{(1)} X_{32}^{(1)}-X_{13}^{(1)} X_{31}^{(1)}\right. \text {, } \\
& \left.X_{23}^{(1)} X_{32}^{(2)}+X_{13}^{(1)} X_{31}^{(2)}, X_{12}^{(1)} X_{23}^{(1)}-X_{13}^{(2)}, X_{23}^{(2)}, X_{22}^{(2)}\right) \cup \\
& \cup V\left(X_{11}^{(1)}, X_{11}^{(2)}, X_{22}^{(1)}, X_{21}^{(1)}, X_{21}^{(2)}, X_{12}^{(2)}, X_{33}^{(1)}, X_{33}^{(2)}-X_{23}^{(1)} X_{32}^{(1)}-X_{13}^{(1)} X_{31}^{(1)}\right. \text {, } \\
& \left.X_{23}^{(2)} X_{32}^{(1)}+X_{13}^{(2)} X_{31}^{(1)}, X_{32}^{(2)}-X_{12}^{(1)} X_{31}^{(1)}, X_{31}^{(2)}, X_{22}^{(2)}\right)
\end{aligned}
$$




\section{Como}

$$
\begin{aligned}
& C_{4}= V\left(X_{11}^{(1)}, X_{11}^{(2)}, X_{22}^{(1)}, X_{12}^{(1)}, X_{21}^{(2)}, X_{12}^{(2)}, X_{33}^{(1)}, X_{33}^{(2)}-X_{23}^{(1)} X_{32}^{(1)}-X_{13}^{(1)} X_{31}^{(1)},\right. \\
&\left.X_{23}^{(2)} X_{32}^{(1)}-X_{13}^{(1)} X_{21}^{(1)} X_{32}^{(1)}+X_{13}^{(1)} X_{31}^{(2)}, X_{32}^{(2)}, X_{13}^{(2)}, X_{22}^{(2)}\right) \\
& C_{5}=V\left(X_{11}^{(1)}, X_{11}^{(2)}, X_{22}^{(1)}, X_{12}^{(1)}, X_{21}^{(2)}, X_{12}^{(2)}, X_{33}^{(1)}, X_{33}^{(2)}-X_{23}^{(1)} X_{32}^{(1)}-X_{13}^{(1)} X_{31}^{(1)},\right. \\
&\left.X_{23}^{(1)} X_{32}^{(2)}+X_{13}^{(1)} X_{31}^{(2)}, X_{23}^{(2)}-X_{13}^{(1)} X_{21}^{(1)}, X_{13}^{(2)}, X_{22}^{(2)}\right), \\
& C_{7}=V\left(X_{11}^{(1)}, X_{11}^{(2)}, X_{22}^{(1)}, X_{12}^{(1)}, X_{21}^{(2)}, X_{12}^{(2)}, X_{33}^{(1)}, X_{33}^{(2)}-X_{23}^{(1)} X_{32}^{(1)}-X_{13}^{(1)} X_{31}^{(1)},\right. \\
&\left.X_{23}^{(1)} X_{32}^{(2)}+X_{23}^{(2)} X_{32}^{(1)}+X_{13}^{(1)} X_{31}^{(2)}+X_{13}^{(2)} X_{31}^{(1)}, X_{23}^{(2)} X_{32}^{(2)}+X_{13}^{(2)} X_{31}^{(2)}, X_{21}^{(1)}, X_{22}^{(2)}\right), \\
& C_{8}=V\left(X_{11}^{(1)}, X_{11}^{(2)}, X_{22}^{(1)}, X_{12}^{(1)}, X_{21}^{(2)}, X_{12}^{(2)}, X_{33}^{(1)}, X_{33}^{(2)}-X_{23}^{(1)} X_{32}^{(1)}-X_{13}^{(1)} X_{31}^{(1)},\right. \\
&\left.X_{23}^{(2)} X_{32}^{(1)}+X_{13}^{(2)} X_{31}^{(1)}, X_{21}^{(1)} X_{32}^{(1)}-X_{31}^{(2)}, X_{32}^{(2)}, X_{22}^{(2)}\right)
\end{aligned}
$$

\section{e denotando}

$$
\begin{aligned}
C_{14}:= & V\left(X_{11}^{(1)}, X_{11}^{(2)}, X_{22}^{(1)}, X_{21}^{(1)}, X_{21}^{(2)}, X_{12}^{(2)}, X_{33}^{(1)}, X_{33}^{(2)}-X_{23}^{(1)} X_{32}^{(1)}-X_{13}^{(1)} X_{31}^{(1)},\right. \\
& \left.X_{23}^{(1)} X_{32}^{(2)}-X_{12}^{(1)} X_{23}^{(1)} X_{31}^{(1)}+X_{13}^{(2)} X_{31}^{(1)}, X_{31}^{(2)}, X_{23}^{(2)}, X_{22}^{(2)}\right) \\
C_{15}:= & V\left(X_{11}^{(1)}, X_{11}^{(2)}, X_{22}^{(1)}, X_{21}^{(1)}, X_{21}^{(2)}, X_{12}^{(2)}, X_{33}^{(1)}, X_{33}^{(2)}-X_{23}^{(1)} X_{32}^{(1)}-X_{13}^{(1)} X_{31}^{(1)},\right. \\
& \left.X_{23}^{(1)} X_{32}^{(2)}+X_{13}^{(1)} X_{31}^{(2)}, X_{12}^{(1)} X_{23}^{(1)}-X_{13}^{(2)}, X_{23}^{(2)}, X_{22}^{(2)}\right), \\
C_{16}:= & V\left(X_{11}^{(1)}, X_{11}^{(2)}, X_{22}^{(1)}, X_{21}^{(1)}, X_{21}^{(2)}, X_{12}^{(2)}, X_{33}^{(1)}, X_{33}^{(2)}-X_{23}^{(1)} X_{32}^{(1)}-X_{13}^{(1)} X_{31}^{(1)},\right. \\
& \left.X_{23}^{(2)} X_{32}^{(1)}+X_{13}^{(2)} X_{31}^{(1)}, X_{32}^{(2)}-X_{12}^{(1)} X_{31}^{(1)}, X_{31}^{(2)}, X_{22}^{(2)}\right),
\end{aligned}
$$

temos

$$
W_{141}=C_{4} \cup C_{5} \cup C_{7} \cup C_{8} \cup C_{14} \cup C_{15} \cup C_{16} .
$$

Por outro lado, as equações que determinam

$$
W_{142}=V\left(X_{11}^{(1)}, X_{11}^{(2)}, X_{22}^{(1)}, p_{22}^{\mathbf{x}}, X_{21}^{(2)}, X_{12}^{(2)}, X_{33}^{(1)}, p_{32}^{\mathbf{X}}, p_{33}^{\mathbf{X}}, p_{34}^{\mathbf{X}}, p_{35}^{\mathbf{X}}, X_{31}^{(2)}\right) \subset k^{18}
$$

são:

$$
\begin{aligned}
\mathbf{X} & =\left\{X_{11}^{(1)}, X_{11}^{(2)}, X_{22}^{(1)}, X_{21}^{(2)}, X_{12}^{(2)}, X_{33}^{(1)}, X_{31}^{(2)}\right\} \\
p_{22}^{\mathbf{X}} & =X_{22}^{(2)}-X_{12}^{(1)} X_{21}^{(1)}, \\
p_{32}^{\mathbf{X}} & =X_{33}^{(2)}-X_{23}^{(1)} X_{32}^{(1)}-X_{13}^{(1)} X_{31}^{(1)}, \\
p_{33}^{\mathbf{X}} & =X_{12}^{(1)} X_{23}^{(1)} X_{31}^{(1)}+X_{13}^{(1)} X_{21}^{(1)} X_{32}^{(1)}-\underbrace{X_{13}^{(1)} X_{31}^{(2)}}_{=0}-X_{23}^{(1)} X_{32}^{(2)}-X_{13}^{(2)} X_{31}^{(1)}-X_{23}^{(2)} X_{32}^{(1)} \\
& =X_{12}^{(1)} X_{23}^{(1)} X_{31}^{(1)}+X_{13}^{(1)} X_{21}^{(1)} X_{32}^{(1)}-X_{23}^{(1)} X_{32}^{(2)}-X_{13}^{(2)} X_{31}^{(1)}-X_{23}^{(2)} X_{32}^{(1)}, \\
p_{34}^{\mathbf{X}} & =-X_{23}^{(2)} X_{32}^{(2)} \underbrace{-X_{13}^{(2)} X_{31}^{(2)}+X_{12}^{(1)} X_{23}^{(1)} X_{31}^{(2)}}_{=0}+X_{13}^{(1)} X_{21}^{(1)} X_{32}^{(2)}-X_{13}^{(1)} X_{22}^{(2)} X_{31}^{(1)}+X_{12}^{(1)} X_{23}^{(2)} X_{31}^{(1)}+X_{13}^{(2)} X_{21}^{(1)} X_{32}^{(1)} \\
& =-X_{23}^{(2)} X_{32}^{(2)}+X_{13}^{(1)} X_{21}^{(1)} X_{32}^{(2)}-X_{13}^{(1)} X_{22}^{(2)} X_{31}^{(1)}+X_{12}^{(1)} X_{23}^{(2)} X_{31}^{(1)}+X_{13}^{(2)} X_{21}^{(1)} X_{32}^{(1)}
\end{aligned}
$$




$$
\begin{aligned}
& =-X_{23}^{(2)} X_{32}^{(2)}+X_{12}^{(1)} X_{23}^{(2)} X_{31}^{(1)}+X_{13}^{(1)} X_{21}^{(1)} X_{32}^{(2)}-X_{13}^{(1)} X_{22}^{(2)} X_{31}^{(1)}+X_{13}^{(2)} X_{21}^{(1)} X_{32}^{(1)} \\
& =-X_{23}^{(2)} X_{32}^{(2)}+X_{12}^{(1)} X_{23}^{(2)} X_{31}^{(1)}+X_{13}^{(1)} X_{21}^{(1)} X_{32}^{(2)}-X_{13}^{(1)} X_{12}^{(1)} X_{21}^{(1)} X_{31}^{(1)}+X_{13}^{(2)} X_{21}^{(1)} X_{32}^{(1)} \\
& =-X_{23}^{(2)}\left(X_{32}^{(2)}-X_{12}^{(1)} X_{31}^{(1)}+X_{13}^{(1)} X_{21}^{(1)}\left(X_{32}^{(2)}-X_{12}^{(1)} X_{31}^{(1)}\right)+X_{13}^{(2)} X_{21}^{(1)} X_{32}^{(1)}\right. \\
& =\left(-X_{23}^{(2)}+X_{13}^{(1)} X_{21}^{(1)}\right)\left(X_{32}^{(2)}-X_{12}^{(1)} X_{31}^{(1)}\right)+X_{13}^{(2)} X_{21}^{(1)} X_{32}^{(1)} \\
& =-\left(X_{23}^{(2)}-X_{13}^{(1)} X_{21}^{(1)}\right)\left(X_{32}^{(2)}-X_{12}^{(1)} X_{31}^{(1)}\right)+X_{13}^{(2)} X_{21}^{(1)} X_{32}^{(1)}, \\
p_{35}^{\mathbf{X}} & =\underbrace{X_{12}^{(1)} X_{23}^{(2)} X_{31}^{(2)}}_{=0}+X_{13}^{(2)} X_{21}^{(1)} X_{32}^{(2)}-X_{13}^{(2)} X_{22}^{(2)} X_{31}^{(1)} \\
& =X_{13}^{(2)} X_{21}^{(1)} X_{32}^{(2)}-X_{13}^{(2)} X_{22}^{(2)} X_{31}^{(1)} \\
& =X_{13}^{(2)} X_{21}^{(1)} X_{32}^{(2)}-X_{13}^{(2)} X_{12}^{(1)} X_{21}^{(1)} X_{31}^{(1)} \\
& =X_{13}^{(2)} X_{21}^{(1)}\left(X_{32}^{(2)}-X_{12}^{(1)} X_{31}^{(1)}\right) .
\end{aligned}
$$

\section{Portanto a decomposição pode ser obtida da seguinte forma}

$$
\begin{aligned}
& W_{142}=V\left(X_{11}^{(1)}, X_{11}^{(2)}, X_{22}^{(1)}, p_{22}^{\mathbf{X}}, X_{21}^{(2)}, X_{12}^{(2)}, X_{33}^{(1)}, p_{32}^{\mathbf{X}}, p_{33}^{\mathbf{X}}, p_{34}^{\mathbf{X}}, p_{35}^{\mathbf{X}}, X_{31}^{(2)}\right) \\
& =V\left(X_{11}^{(1)}, X_{11}^{(2)}, X_{22}^{(1)}, X_{22}^{(2)}-X_{12}^{(1)} X_{21}^{(1)}, X_{21}^{(2)}, X_{12}^{(2)}, X_{33}^{(1)}, X_{33}^{(2)}-X_{23}^{(1)} X_{32}^{(1)}-X_{13}^{(1)} X_{31}^{(1)}\right. \text {, } \\
& -X_{23}^{(1)} X_{32}^{(2)}-X_{23}^{(2)} X_{32}^{(1)}+X_{12}^{(1)} X_{23}^{(1)} X_{31}^{(1)}+X_{13}^{(1)} X_{21}^{(1)} X_{32}^{(1)}-X_{13}^{(2)} X_{31}^{(1)} \text {, } \\
& \left.\left(X_{23}^{(2)}-X_{13}^{(1)} X_{21}^{(1)}\right)\left(X_{32}^{(2)}-X_{12}^{(1)} X_{31}^{(1)}\right)-X_{13}^{(2)} X_{21}^{(1)} X_{32}^{(1)}, X_{13}^{(2)} X_{21}^{(1)}\left(X_{32}^{(2)}-X_{12}^{(1)} X_{31}^{(1)}\right), X_{31}^{(2)}\right) \\
& =V\left(X_{11}^{(1)}, X_{11}^{(2)}, X_{22}^{(1)}, X_{22}^{(2)}-X_{12}^{(1)} X_{21}^{(1)}, X_{21}^{(2)}, X_{12}^{(2)}, X_{33}^{(1)}, X_{33}^{(2)}-X_{23}^{(1)} X_{32}^{(1)}-X_{13}^{(1)} X_{31}^{(1)}\right. \text {, } \\
& \left.X_{12}^{(1)} X_{23}^{(1)} X_{31}^{(1)}+X_{13}^{(1)} X_{21}^{(1)} X_{32}^{(1)}-X_{23}^{(1)} X_{32}^{(2)}-X_{23}^{(2)} X_{32}^{(1)},\left(X_{23}^{(2)}-X_{13}^{(1)} X_{21}^{(1)}\right)\left(X_{32}^{(2)}-X_{12}^{(1)} X_{31}^{(1)}\right), X_{13}^{(2)}, X_{31}^{(2)}\right) \cup \\
& \cup V\left(X_{11}^{(1)}, X_{11}^{(2)}, X_{22}^{(1)}, X_{22}^{(2)}, X_{21}^{(2)}, X_{12}^{(2)}, X_{33}^{(1)}, X_{33}^{(2)}-X_{23}^{(1)} X_{32}^{(1)}-X_{13}^{(1)} X_{31}^{(1)}\right. \text {, } \\
& \left.X_{23}^{(1)} X_{32}^{(2)}+X_{23}^{(2)} X_{32}^{(1)}-X_{12}^{(1)} X_{23}^{(1)} X_{31}^{(1)}+X_{13}^{(2)} X_{31}^{(1)}, X_{23}^{(2)}\left(X_{32}^{(2)}-X_{12}^{(1)} X_{31}^{(1)}\right), X_{21}^{(1)}, X_{31}^{(2)}\right) \cup \\
& \cup V\left(X_{11}^{(1)}, X_{11}^{(2)}, X_{22}^{(1)}, X_{22}^{(2)}-X_{12}^{(1)} X_{21}^{(1)}, X_{21}^{(2)}, X_{12}^{(2)}, X_{33}^{(1)}, X_{33}^{(2)}-X_{23}^{(1)} X_{32}^{(1)}-X_{13}^{(1)} X_{31}^{(1)}\right. \text {, } \\
& \left.X_{23}^{(2)} X_{32}^{(1)}-X_{13}^{(1)} X_{21}^{(1)} X_{32}^{(1)}+X_{13}^{(2)} X_{31}^{(1)}, X_{13}^{(2)} X_{21}^{(1)} X_{32}^{(1)}, X_{32}^{(2)}-X_{12}^{(1)} X_{31}^{(1)}, X_{31}^{(2)}\right) \\
& =V\left(X_{11}^{(1)}, X_{11}^{(2)}, X_{22}^{(1)}, X_{22}^{(2)}-X_{12}^{(1)} X_{21}^{(1)}, X_{21}^{(2)}, X_{12}^{(2)}, X_{33}^{(1)}, X_{33}^{(2)}-X_{23}^{(1)} X_{32}^{(1)}-X_{13}^{(1)} X_{31}^{(1)}\right. \text {, } \\
& \left.X_{23}^{(1)} X_{32}^{(2)}-X_{12}^{(1)} X_{23}^{(1)} X_{31}^{(1)}, X_{23}^{(2)}-X_{13}^{(1)} X_{21}^{(1)}, X_{13}^{(2)}, X_{31}^{(2)}\right) \cup \\
& \cup V\left(X_{11}^{(1)}, X_{11}^{(2)}, X_{22}^{(1)}, X_{22}^{(2)}-X_{12}^{(1)} X_{21}^{(1)}, X_{21}^{(2)}, X_{12}^{(2)}, X_{33}^{(1)}, X_{33}^{(2)}-X_{23}^{(1)} X_{32}^{(1)}-X_{13}^{(1)} X_{31}^{(1)}\right. \text {, } \\
& \left.X_{23}^{(2)} X_{32}^{(1)}-X_{13}^{(1)} X_{21}^{(1)} X_{32}^{(1)}, X_{32}^{(2)}-X_{12}^{(1)} X_{31}^{(1)}, X_{13}^{(2)}, X_{31}^{(2)}\right) \cup \\
& \cup V\left(X_{11}^{(1)}, X_{11}^{(2)}, X_{22}^{(1)}, X_{22}^{(2)}, X_{21}^{(2)}, X_{12}^{(2)}, X_{33}^{(1)}, X_{33}^{(2)}-X_{23}^{(1)} X_{32}^{(1)}-X_{13}^{(1)} X_{31}^{(1)}\right. \text {, } \\
& \left.X_{23}^{(1)} X_{32}^{(2)}-X_{12}^{(1)} X_{23}^{(1)} X_{31}^{(1)}+X_{13}^{(2)} X_{31}^{(1)}, X_{23}^{(2)}, X_{21}^{(1)}, X_{31}^{(2)}\right) \cup \\
& \cup V\left(X_{11}^{(1)}, X_{11}^{(2)}, X_{22}^{(1)}, X_{22}^{(2)}, X_{21}^{(2)}, X_{12}^{(2)}, X_{33}^{(1)}, X_{33}^{(2)}-X_{23}^{(1)} X_{32}^{(1)}-X_{13}^{(1)} X_{31}^{(1)}\right. \text {, } \\
& \left.X_{23}^{(2)} X_{32}^{(1)}+X_{13}^{(2)} X_{31}^{(1)}, X_{32}^{(2)}-X_{12}^{(1)} X_{31}^{(1)}, X_{21}^{(1)}, X_{31}^{(2)}\right) \cup
\end{aligned}
$$




$$
\begin{aligned}
& \cup V\left(X_{11}^{(1)}, X_{11}^{(2)}, X_{22}^{(1)}, X_{22}^{(2)}-X_{12}^{(1)} X_{21}^{(1)}, X_{21}^{(2)}, X_{12}^{(2)}, X_{33}^{(1)}, X_{33}^{(2)}-X_{13}^{(1)} X_{31}^{(1)}, X_{13}^{(2)} X_{31}^{(1)},\right. \\
& \left.X_{32}^{(1)}, X_{32}^{(2)}-X_{12}^{(1)} X_{31}^{(1)}, X_{31}^{(2)}\right) \\
& =V\left(X_{11}^{(1)}, X_{11}^{(2)}, X_{22}^{(1)}, X_{22}^{(2)}-X_{12}^{(1)} X_{21}^{(1)}, X_{21}^{(2)}, X_{12}^{(2)}, X_{33}^{(1)}, X_{33}^{(2)}-X_{13}^{(1)} X_{31}^{(1)}, X_{23}^{(1)}\right. \text {, } \\
& \left.X_{23}^{(2)}-X_{13}^{(1)} X_{21}^{(1)}, X_{13}^{(2)}, X_{31}^{(2)}\right) \cup \\
& \cup V\left(X_{11}^{(1)}, X_{11}^{(2)}, X_{22}^{(1)}, X_{22}^{(2)}-X_{12}^{(1)} X_{21}^{(1)}, X_{21}^{(2)}, X_{12}^{(2)}, X_{33}^{(1)}, X_{33}^{(2)}-X_{23}^{(1)} X_{32}^{(1)}-X_{13}^{(1)} X_{31}^{(1)}\right. \text {, } \\
& \left.X_{32}^{(2)}-X_{12}^{(1)} X_{31}^{(1)}, X_{23}^{(2)}-X_{13}^{(1)} X_{21}^{(1)}, X_{13}^{(2)}, X_{31}^{(2)}\right) \cup \\
& \cup V\left(X_{11}^{(1)}, X_{11}^{(2)}, X_{22}^{(1)}, X_{22}^{(2)}-X_{12}^{(1)} X_{21}^{(1)}, X_{21}^{(2)}, X_{12}^{(2)}, X_{33}^{(1)}, X_{33}^{(2)}-X_{13}^{(1)} X_{31}^{(1)}, X_{32}^{(1)}\right. \text {, } \\
& \left.X_{32}^{(2)}-X_{12}^{(1)} X_{31}^{(1)}, X_{13}^{(2)}, X_{31}^{(2)}\right) \cup \\
& \cup V\left(X_{11}^{(1)}, X_{11}^{(2)}, X_{22}^{(1)}, X_{22}^{(2)}-X_{12}^{(1)} X_{21}^{(1)}, X_{21}^{(2)}, X_{12}^{(2)}, X_{33}^{(1)}, X_{33}^{(2)}-X_{23}^{(1)} X_{32}^{(1)}-X_{13}^{(1)} X_{31}^{(1)}\right. \text {, } \\
& \left.X_{23}^{(2)}-X_{13}^{(1)} X_{21}^{(1)}, X_{32}^{(2)}-X_{12}^{(1)} X_{31}^{(1)}, X_{13}^{(2)}, X_{31}^{(2)}\right) \cup \\
& \cup V\left(X_{11}^{(1)}, X_{11}^{(2)}, X_{22}^{(1)}, X_{22}^{(2)}, X_{21}^{(2)}, X_{12}^{(2)}, X_{33}^{(1)}, X_{33}^{(2)}-X_{23}^{(1)} X_{32}^{(1)}-X_{13}^{(1)} X_{31}^{(1)}\right. \text {, } \\
& \left.X_{23}^{(1)} X_{32}^{(2)}-X_{12}^{(1)} X_{23}^{(1)} X_{31}^{(1)}+X_{13}^{(2)} X_{31}^{(1)}, X_{23}^{(2)}, X_{21}^{(1)}, X_{31}^{(2)}\right) \cup \\
& \cup V\left(X_{11}^{(1)}, X_{11}^{(2)}, X_{22}^{(1)}, X_{22}^{(2)}, X_{21}^{(2)}, X_{12}^{(2)}, X_{33}^{(1)}, X_{33}^{(2)}-X_{23}^{(1)} X_{32}^{(1)}-X_{13}^{(1)} X_{31}^{(1)}\right. \text {, } \\
& \left.X_{23}^{(2)} X_{32}^{(1)}+X_{13}^{(2)} X_{31}^{(1)}, X_{32}^{(2)}-X_{12}^{(1)} X_{31}^{(1)}, X_{21}^{(1)}, X_{31}^{(2)}\right) \cup \\
& \cup V\left(X_{11}^{(1)}, X_{11}^{(2)}, X_{22}^{(1)}, X_{22}^{(2)}-X_{12}^{(1)} X_{21}^{(1)}, X_{21}^{(2)}, X_{12}^{(2)}, X_{33}^{(1)}, X_{33}^{(2)}-X_{13}^{(1)} X_{31}^{(1)}, X_{13}^{(2)}, X_{32}^{(1)}, X_{32}^{(2)}-X_{12}^{(1)} X_{31}^{(1)}, X_{31}^{(2)}\right) \cup \\
& \cup V\left(X_{11}^{(1)}, X_{11}^{(2)}, X_{22}^{(1)}, X_{22}^{(2)}-X_{12}^{(1)} X_{21}^{(1)}, X_{21}^{(2)}, X_{12}^{(2)}, X_{33}^{(1)}, X_{33}^{(2)}, X_{31}^{(1)}, X_{32}^{(1)}, X_{32}^{(2)}, X_{31}^{(2)}\right) \\
& =V\left(X_{11}^{(1)}, X_{11}^{(2)}, X_{22}^{(1)}, X_{22}^{(2)}-X_{12}^{(1)} X_{21}^{(1)}, X_{21}^{(2)}, X_{12}^{(2)}, X_{33}^{(1)}, X_{33}^{(2)}-X_{13}^{(1)} X_{31}^{(1)}, X_{23}^{(1)}, X_{23}^{(2)}-X_{13}^{(1)} X_{21}^{(1)}, X_{13}^{(2)}, X_{31}^{(2)}\right) \cup \\
& \cup V\left(X_{11}^{(1)}, X_{11}^{(2)}, X_{22}^{(1)}, X_{22}^{(2)}-X_{12}^{(1)} X_{21}^{(1)}, X_{21}^{(2)}, X_{12}^{(2)}, X_{33}^{(1)}, X_{33}^{(2)}-X_{23}^{(1)} X_{32}^{(1)}-X_{13}^{(1)} X_{31}^{(1)}\right. \text {, } \\
& \left.X_{32}^{(2)}-X_{12}^{(1)} X_{31}^{(1)}, X_{23}^{(2)}-X_{13}^{(1)} X_{21}^{(1)}, X_{13}^{(2)}, X_{31}^{(2)}\right) \cup \\
& \cup V\left(X_{11}^{(1)}, X_{11}^{(2)}, X_{22}^{(1)}, X_{22}^{(2)}-X_{12}^{(1)} X_{21}^{(1)}, X_{21}^{(2)}, X_{12}^{(2)}, X_{33}^{(1)}, X_{33}^{(2)}-X_{13}^{(1)} X_{31}^{(1)}, X_{32}^{(1)}, X_{32}^{(2)}-X_{12}^{(1)} X_{31}^{(1)}, X_{13}^{(2)}, X_{31}^{(2)}\right) \cup \\
& \cup V\left(X_{11}^{(1)}, X_{11}^{(2)}, X_{22}^{(1)}, X_{22}^{(2)}, X_{21}^{(2)}, X_{12}^{(2)}, X_{33}^{(1)}, X_{33}^{(2)}-X_{23}^{(1)} X_{32}^{(1)}-X_{13}^{(1)} X_{31}^{(1)}\right. \text {, } \\
& \left.X_{23}^{(1)} X_{32}^{(2)}-X_{12}^{(1)} X_{23}^{(1)} X_{31}^{(1)}+X_{13}^{(2)} X_{31}^{(1)}, X_{23}^{(2)}, X_{21}^{(1)}, X_{31}^{(2)}\right) \cup \\
& \cup V\left(X_{11}^{(1)}, X_{11}^{(2)}, X_{22}^{(1)}, X_{22}^{(2)}, X_{21}^{(2)}, X_{12}^{(2)}, X_{33}^{(1)}, X_{33}^{(2)}-X_{23}^{(1)} X_{32}^{(1)}-X_{13}^{(1)} X_{31}^{(1)}\right. \text {, } \\
& \left.X_{23}^{(2)} X_{32}^{(1)}+X_{13}^{(2)} X_{31}^{(1)}, X_{32}^{(2)}-X_{12}^{(1)} X_{31}^{(1)}, X_{21}^{(1)}, X_{31}^{(2)}\right) \cup \\
& \cup V\left(X_{11}^{(1)}, X_{11}^{(2)}, X_{22}^{(1)}, X_{22}^{(2)}-X_{12}^{(1)} X_{21}^{(1)}, X_{21}^{(2)}, X_{12}^{(2)}, X_{33}^{(1)}, X_{33}^{(2)}, X_{31}^{(1)}, X_{32}^{(1)}, X_{32}^{(2)}, X_{31}^{(2)}\right) \text {. }
\end{aligned}
$$


Note que

$$
\begin{array}{rl}
C_{10}= & V\left(X_{11}^{(1)}, X_{11}^{(2)}, X_{22}^{(1)}, X_{22}^{(2)}-X_{12}^{(1)} X_{21}^{(1)}, X_{21}^{(2)}, X_{12}^{(2)}, X_{33}^{(1)}, X_{33}^{(2)}-X_{13}^{(1)} X_{31}^{(1)}, X_{23}^{(1)},\right. \\
& \left.X_{23}^{(2)}-X_{13}^{(1)} X_{21}^{(1)}, X_{13}^{(2)}, X_{31}^{(2)}\right), \\
C_{11}= & V\left(X_{11}^{(1)}, X_{11}^{(2)}, X_{22}^{(1)}, X_{22}^{(2)}-X_{12}^{(1)} X_{21}^{(1)}, X_{21}^{(2)}, X_{12}^{(2)}, X_{33}^{(1)}, X_{33}^{(2)}-X_{23}^{(1)} X_{32}^{(1)}-X_{13}^{(1)} X_{31}^{(1)},\right. \\
& \left.X_{32}^{(2)}-X_{12}^{(1)} X_{31}^{(1)}, X_{23}^{(2)}-X_{13}^{(1)} X_{21}^{(1)}, X_{13}^{(2)}, X_{31}^{(2)}\right), \\
C_{12}=V & V\left(X_{11}^{(1)}, X_{11}^{(2)}, X_{22}^{(1)}, X_{22}^{(2)}-X_{12}^{(1)} X_{21}^{(1)}, X_{21}^{(2)}, X_{12}^{(2)}, X_{33}^{(1)}, X_{33}^{(2)}-X_{13}^{(1)} X_{31}^{(1)}, X_{32}^{(1)},\right. \\
& \left.X_{32}^{(2)}-X_{12}^{(1)} X_{31}^{(1)}, X_{13}^{(2)}, X_{31}^{(2)}\right), \\
C_{14}=V & V\left(X_{11}^{(1)}, X_{11}^{(2)}, X_{22}^{(1)}, X_{22}^{(2)}, X_{21}^{(2)}, X_{12}^{(2)}, X_{33}^{(1)}, X_{33}^{(2)}-X_{23}^{(1)} X_{32}^{(1)}-X_{13}^{(1)} X_{31}^{(1)},\right. \\
& \left.X_{23}^{(1)} X_{32}^{(2)}-X_{12}^{(1)} X_{23}^{(1)} X_{31}^{(1)}+X_{13}^{(2)} X_{31}^{(1)}, X_{23}^{(2)}, X_{21}^{(1)}, X_{31}^{(2)}\right), \\
C_{16}=V\left(X_{11}^{(1)}, X_{11}^{(2)}, X_{22}^{(1)}, X_{22}^{(2)}, X_{21}^{(2)}, X_{12}^{(2)}, X_{33}^{(1)}, X_{33}^{(2)}-X_{23}^{(1)} X_{32}^{(1)}-X_{13}^{(1)} X_{31}^{(1)},\right. \\
& \left.X_{23}^{(2)} X_{32}^{(1)}+X_{13}^{(2)} X_{31}^{(1)}, X_{32}^{(2)}-X_{12}^{(1)} X_{31}^{(1)}, X_{21}^{(1)}, X_{31}^{(2)}\right)
\end{array}
$$

e denotando

$$
C_{17}:=V\left(X_{11}^{(1)}, X_{11}^{(2)}, X_{22}^{(1)}, X_{22}^{(2)}-X_{12}^{(1)} X_{21}^{(1)}, X_{21}^{(2)}, X_{12}^{(2)}, X_{33}^{(1)}, X_{33}^{(2)}, X_{31}^{(1)}, X_{32}^{(1)}, X_{32}^{(2)}, X_{31}^{(2)}\right),
$$

tem-se

$$
W_{142}=C_{10} \cup C_{11} \cup C_{12} \cup C_{14} \cup C_{16} \cup C_{17} .
$$

Pelas equações (B.11) e (B.12), segue que

$$
W_{14}=C_{4} \cup C_{5} \cup C_{7} \cup C_{8} \cup C_{10} \cup C_{11} \cup C_{12} \cup C_{14} \cup C_{15} \cup C_{16} \cup C_{17} .
$$

Finalmente, das equações (B.4), (B.7), (B.10) e (B.13) temos que,

$$
W_{1}=\bigcup_{i=1}^{17} C_{i} .
$$

\section{B.3 Decomposição de $W_{2}$}

Para determinar a decomposição de $W_{2}$ podemos fazer o mesmo raciocinio feito com a variedade $W_{1}$, mas, neste caso consideraremos o automorfismo $\tau$ definido por

$$
\tau\left(X_{i j}^{p}\right)=X_{j i}^{p}, \quad i, j=1,2,3 \quad p=1,2 \text {. }
$$

Claramente $W_{2}=\tau\left(W_{1}\right)$, pois

$$
W_{1}=V\left(X_{11}^{(1)}, X_{11}^{(2)}, X_{22}^{(1)}, p_{22}^{\mathbf{x}}, p_{23}^{\mathbf{X}}, X_{12}^{(2)}, X_{33}^{(1)}, p_{32}^{\mathbf{x}}, p_{33}^{\mathbf{X}}, p_{34}^{\mathbf{x}}, p_{35}^{\mathbf{X}}, p_{36}^{\mathbf{x}}\right) \subset k^{18},
$$

com

$$
\begin{aligned}
\mathbf{X} & =\left\{X_{11}^{(1)}, X_{11}^{(2)}, X_{22}^{(1)}, X_{12}^{(2)}, X_{33}^{(1)}\right\}, \\
p_{22}^{\mathbf{X}} & =X_{22}^{(2)}-X_{12}^{(1)} X_{21}^{(1)},
\end{aligned}
$$




$$
\begin{aligned}
p_{23}^{\mathbf{X}}= & X_{12}^{(1)} X_{21}^{(2)}, \\
p_{32}^{\mathbf{X}}= & X_{33}^{(2)}-X_{23}^{(1)} X_{32}^{(1)}-X_{13}^{(1)} X_{31}^{(1)}, \\
p_{33}^{\mathbf{X}}= & X_{12}^{(1)} X_{23}^{(1)} X_{31}^{(1)}+X_{13}^{(1)} X_{21}^{(1)} X_{32}^{(1)}-X_{13}^{(1)} X_{31}^{(2)}-X_{23}^{(1)} X_{32}^{(2)}-X_{13}^{(2)} X_{31}^{(1)}-X_{23}^{(2)} X_{32}^{(1)}, \\
p_{34}^{\mathbf{X}}= & -X_{23}^{(2)} X_{32}^{(2)}-X_{13}^{(2)} X_{31}^{(2)}+X_{12}^{(1)} X_{23}^{(1)} X_{31}^{(2)}+X_{13}^{(1)} X_{21}^{(1)} X_{32}^{(2)}-X_{13}^{(1)} X_{22}^{(2)} X_{31}^{(1)}+ \\
& +X_{12}^{(1)} X_{23}^{(2)} X_{31}^{(1)}+X_{13}^{(1)} X_{21}^{(2)} X_{32}^{(1)}+X_{13}^{(2)} X_{21}^{(1)} X_{32}^{(1)}, \\
p_{35}^{\mathbf{X}}= & -X_{13}^{(1)} X_{22}^{(2)} X_{31}^{(2)}+X_{12}^{(1)} X_{23}^{(2)} X_{31}^{(2)}+X_{13}^{(1)} X_{21}^{(2)} X_{32}^{(2)}+X_{13}^{(2)} X_{21}^{(1)} X_{32}^{(2)}-X_{13}^{(2)} X_{22}^{(2)} X_{31}^{(1)}+X_{13}^{(2)} X_{21}^{(2)} X_{32}^{(1)}, \\
p_{36}^{\mathbf{X}}= & X_{13}^{(2)}\left(X_{21}^{(2)} X_{32}^{(2)}-X_{22}^{(2)} X_{31}^{(2)}\right)
\end{aligned}
$$

e

$$
W_{2}=V\left(X_{11}^{(1)}, X_{11}^{(2)}, X_{22}^{(1)}, p_{22}^{\mathbf{x}}, p_{23}^{\mathbf{X}}, X_{21}^{(2)}, X_{33}^{(1)}, p_{32}^{\mathbf{x}}, p_{33}^{\mathbf{x}}, p_{34}^{\mathbf{X}}, p_{35}^{\mathbf{X}}, p_{36}^{\mathbf{X}}\right) \subset k^{18},
$$

com

$$
\begin{aligned}
& \mathbf{X}=\left\{X_{11}^{(1)}, X_{11}^{(2)}, X_{22}^{(1)}, X_{21}^{(2)}, X_{33}^{(1)}\right\}, \\
& p_{22}^{\mathbf{x}}=X_{22}^{(2)}-X_{12}^{(1)} X_{21}^{(1)} \text {, } \\
& p_{23}^{\mathbf{X}}=\underbrace{X_{12}^{(1)} X_{21}^{(2)}}_{=0}+X_{12}^{(2)} X_{21}^{(1)} \\
& =X_{12}^{(2)} X_{21}^{(1)} \text {, } \\
& p_{32}^{\mathbf{X}}=X_{33}^{(2)}-X_{23}^{(1)} X_{32}^{(1)}-X_{13}^{(1)} X_{31}^{(1)} \text {, } \\
& p_{33}^{\mathbf{X}}=X_{12}^{(1)} X_{23}^{(1)} X_{31}^{(1)}+X_{13}^{(1)} X_{21}^{(1)} X_{32}^{(1)}-X_{13}^{(1)} X_{31}^{(2)}-X_{23}^{(1)} X_{32}^{(2)}-X_{13}^{(2)} X_{31}^{(1)}-X_{23}^{(2)} X_{32}^{(1)} \text {, } \\
& p_{34}^{\mathbf{X}}=-X_{23}^{(2)} X_{32}^{(2)}-X_{13}^{(2)} X_{31}^{(2)}+X_{12}^{(1)} X_{23}^{(1)} X_{31}^{(2)}+X_{13}^{(1)} X_{21}^{(1)} X_{32}^{(2)}-X_{13}^{(1)} X_{22}^{(2)} X_{31}^{(1)}+ \\
& +X_{12}^{(1)} X_{23}^{(2)} X_{31}^{(1)}+\underbrace{X_{13}^{(1)} X_{21}^{(2)} X_{32}^{(1)}}_{=0}+X_{12}^{(2)} X_{23}^{(1)} X_{31}^{(1)}+X_{13}^{(2)} X_{21}^{(1)} X_{32}^{(1)} \\
& =-X_{23}^{(2)} X_{32}^{(2)}-X_{13}^{(2)} X_{31}^{(2)}+X_{12}^{(1)} X_{23}^{(1)} X_{31}^{(2)}+X_{13}^{(1)} X_{21}^{(1)} X_{32}^{(2)}-X_{13}^{(1)} X_{22}^{(2)} X_{31}^{(1)}+ \\
& +X_{12}^{(1)} X_{23}^{(2)} X_{31}^{(1)}+X_{12}^{(2)} X_{23}^{(1)} X_{31}^{(1)}+X_{13}^{(2)} X_{21}^{(1)} X_{32}^{(1)} \text {, } \\
& p_{35}^{\mathbf{X}}=-X_{13}^{(1)} X_{22}^{(2)} X_{31}^{(2)}+X_{12}^{(1)} X_{23}^{(2)} X_{31}^{(2)}+\underbrace{X_{13}^{(1)} X_{21}^{(2)} X_{32}^{(2)}}_{=0}+X_{12}^{(2)} X_{23}^{(1)} X_{31}^{(2)}+X_{13}^{(2)} X_{21}^{(1)} X_{32}^{(2)}- \\
& -X_{13}^{(2)} X_{22}^{(2)} X_{31}^{(1)}+X_{12}^{(2)} X_{23}^{(2)} X_{31}^{(1)}+\underbrace{X_{13}^{(2)} X_{21}^{(2)} X_{32}^{(1)}}_{=0} \\
& =-X_{13}^{(1)} X_{22}^{(2)} X_{31}^{(2)}+X_{12}^{(1)} X_{23}^{(2)} X_{31}^{(2)}+X_{12}^{(2)} X_{23}^{(1)} X_{31}^{(2)}+X_{13}^{(2)} X_{21}^{(1)} X_{32}^{(2)}-X_{13}^{(2)} X_{22}^{(2)} X_{31}^{(1)}+X_{12}^{(2)} X_{23}^{(2)} X_{31}^{(1)} \text {, } \\
& p_{36}^{\mathbf{X}}=-X_{13}^{(2)} X_{22}^{(2)} X_{31}^{(2)}+X_{12}^{(2)} X_{23}^{(2)} X_{31}^{(2)}+\underbrace{X_{13}^{(2)} X_{21}^{(2)} X_{32}^{(2)}}_{=0} \\
& =-X_{13}^{(2)} X_{22}^{(2)} X_{31}^{(2)}+X_{12}^{(2)} X_{23}^{(2)} X_{31}^{(2)} \\
& =\left(X_{12}^{(2)} X_{23}^{(2)}-X_{13}^{(2)} X_{22}^{(2)}\right) X_{31}^{(2)} \text {. }
\end{aligned}
$$

\section{Portanto}

$$
W_{2}=\bigcup_{i=1}^{17} \tau\left(C_{i}\right)
$$

e

$$
\begin{aligned}
& \tau\left(C_{1}\right)=V\left(X_{11}^{(1)}, X_{11}^{(2)}, X_{22}^{(1)}, X_{22}^{(2)}, X_{21}^{(1)}, X_{21}^{(2)}, X_{33}^{(1)}, X_{33}^{(2)}, X_{32}^{(1)}, X_{32}^{(2)}, X_{31}^{(1)}, X_{31}^{(2)}\right), \\
& \tau\left(C_{2}\right)=V\left(X_{11}^{(1)}, X_{11}^{(2)}, X_{22}^{(1)}, X_{22}^{(2)}, X_{21}^{(1)}, X_{21}^{(2)}, X_{33}^{(1)}, X_{33}^{(2)}-X_{32}^{(1)} X_{23}^{(1)}, X_{23}^{(2)}, X_{32}^{(2)}, X_{31}^{(1)}, X_{31}^{(2)}\right), \\
& \tau\left(C_{3}\right)=V\left(X_{11}^{(1)}, X_{11}^{(2)}, X_{22}^{(1)}, X_{22}^{(2)}, X_{21}^{(1)}, X_{21}^{(2)}, X_{33}^{(1)}, X_{33}^{(2)}, X_{23}^{(1)}, X_{23}^{(2)}, X_{31}^{(1)}, X_{31}^{(2)}\right),
\end{aligned}
$$




$$
\begin{aligned}
& \tau\left(C_{4}\right)=V\left(X_{11}^{(1)}, X_{11}^{(2)}, X_{22}^{(1)}, X_{22}^{(2)}, X_{21}^{(1)}, X_{21}^{(2)}, X_{33}^{(1)}, X_{33}^{(2)}-X_{32}^{(1)} X_{23}^{(1)}-X_{31}^{(1)} X_{13}^{(1)},\right. \\
& \left.-X_{32}^{(2)} X_{23}^{(1)}+X_{31}^{(1)} X_{12}^{(1)} X_{23}^{(1)}-X_{31}^{(1)} X_{13}^{(2)}, X_{23}^{(2)}, X_{12}^{(2)}, X_{31}^{(2)}\right), \\
& \tau\left(C_{5}\right)=V\left(X_{11}^{(1)}, X_{11}^{(2)}, X_{22}^{(1)}, X_{22}^{(2)}, X_{21}^{(1)}, X_{21}^{(2)}, X_{33}^{(1)}, X_{33}^{(2)}-X_{32}^{(1)} X_{23}^{(1)}-X_{31}^{(1)} X_{13}^{(1)}\right. \text {, } \\
& \left.-X_{32}^{(1)} X_{23}^{(2)}-X_{31}^{(1)} X_{13}^{(2)},-X_{32}^{(2)}+X_{31}^{(1)} X_{12}^{(1)}, X_{12}^{(2)}, X_{31}^{(2)}\right) \text {, } \\
& \tau\left(C_{6}\right)=V\left(X_{11}^{(1)}, X_{11}^{(2)}, X_{22}^{(1)}, X_{22}^{(2)}, X_{21}^{(1)}, X_{21}^{(2)}, X_{33}^{(1)}, X_{33}^{(2)}-X_{31}^{(1)} X_{13}^{(1)}, X_{13}^{(2)}, X_{23}^{(1)}, X_{23}^{(2)}, X_{31}^{(2)}\right) \text {, } \\
& \tau\left(C_{7}\right)=V\left(X_{11}^{(1)}, X_{11}^{(2)}, X_{22}^{(1)}, X_{22}^{(2)}, X_{21}^{(1)}, X_{21}^{(2)}, X_{33}^{(1)}, X_{33}^{(2)}-X_{32}^{(1)} X_{23}^{(1)}-X_{31}^{(1)} X_{13}^{(1)},\right. \\
& \left.X_{32}^{(1)} X_{23}^{(2)}+X_{32}^{(2)} X_{23}^{(1)}+X_{31}^{(1)} X_{13}^{(2)}+X_{31}^{(2)} X_{13}^{(1)}, X_{32}^{(2)} X_{23}^{(2)}+X_{31}^{(2)} X_{13}^{(2)}, X_{12}^{(1)}, X_{12}^{(2)}\right) \text {, } \\
& \tau\left(C_{8}\right)=V\left(X_{11}^{(1)}, X_{11}^{(2)}, X_{22}^{(1)}, X_{22}^{(2)}, X_{21}^{(1)}, X_{21}^{(2)}, X_{33}^{(1)}, X_{33}^{(2)}-X_{32}^{(1)} X_{23}^{(1)}-X_{31}^{(1)} X_{13}^{(1)}\right. \text {, } \\
& \left.X_{32}^{(2)} X_{23}^{(1)}+X_{31}^{(2)} X_{13}^{(1)}, X_{12}^{(1)} X_{23}^{(1)}-X_{13}^{(2)}, X_{23}^{(2)}, X_{12}^{(2)}\right) \text {, } \\
& \tau\left(C_{9}\right)=V\left(X_{11}^{(1)}, X_{11}^{(2)}, X_{22}^{(1)}, X_{22}^{(2)}, X_{21}^{(1)}, X_{21}^{(2)}, X_{33}^{(1)}, X_{33}^{(2)}, X_{13}^{(1)}, X_{13}^{(2)}, X_{23}^{(1)}, X_{23}^{(2)}\right) \text {, } \\
& \tau\left(C_{10}\right)=V\left(X_{11}^{(1)}, X_{11}^{(2)}, X_{22}^{(1)}, X_{22}^{(2)}-X_{21}^{(1)} X_{12}^{(1)}, X_{12}^{(2)}, X_{21}^{(2)}, X_{33}^{(1)}, X_{33}^{(2)}-X_{31}^{(1)} X_{13}^{(1)}, X_{32}^{(1)}, X_{32}^{(2)}-X_{31}^{(1)} X_{12}^{(1)}, X_{13}^{(2)}, X_{31}^{(2)}\right) \text {, } \\
& \tau\left(C_{11}\right)=V\left(X_{11}^{(1)}, X_{11}^{(2)}, X_{22}^{(1)}, X_{22}^{(2)}-X_{21}^{(1)} X_{12}^{(1)}, X_{12}^{(2)}, X_{21}^{(2)}, X_{33}^{(1)}, X_{33}^{(2)}-X_{32}^{(1)} X_{23}^{(1)}-X_{31}^{(1)} X_{13}^{(1)},\right. \\
& \left.X_{23}^{(2)}-X_{21}^{(1)} X_{13}^{(1)}, X_{32}^{(2)}-X_{31}^{(1)} X_{12}^{(1)}, X_{13}^{(2)}, X_{31}^{(2)}\right) \text {, } \\
& \tau\left(C_{12}\right)=V\left(X_{11}^{(1)}, X_{11}^{(2)}, X_{22}^{(1)}, X_{22}^{(2)}-X_{21}^{(1)} X_{12}^{(1)}, X_{12}^{(2)}, X_{21}^{(2)}, X_{33}^{(1)}, X_{33}^{(2)}-X_{31}^{(1)} X_{13}^{(1)}, X_{23}^{(1)}, X_{23}^{(2)}-X_{21}^{(1)} X_{13}^{(1)}, X_{13}^{(2)}, X_{31}^{(2)}\right), \\
& \tau\left(C_{13}\right)=V\left(X_{11}^{(1)}, X_{11}^{(2)}, X_{22}^{(1)}, X_{22}^{(2)}-X_{21}^{(1)} X_{12}^{(1)}, X_{12}^{(2)}, X_{21}^{(2)}, X_{33}^{(1)}, X_{33}^{(2)}, X_{31}^{(1)}, X_{32}^{(1)}, X_{32}^{(2)}, X_{31}^{(2)}\right), \\
& \tau\left(C_{14}\right)=V\left(X_{11}^{(1)}, X_{11}^{(2)}, X_{22}^{(1)}, X_{12}^{(1)}, X_{12}^{(2)}, X_{21}^{(2)}, X_{33}^{(1)}, X_{33}^{(2)}-X_{32}^{(1)} X_{23}^{(1)}-X_{31}^{(1)} X_{13}^{(1)},\right. \\
& \left.X_{32}^{(1)} X_{23}^{(2)}-X_{21}^{(1)} X_{32}^{(1)} X_{13}^{(1)}+X_{31}^{(2)} X_{13}^{(1)}, X_{13}^{(2)}, X_{32}^{(2)}, X_{22}^{(2)}\right) \text {, } \\
& \tau\left(C_{15}\right)=V\left(X_{11}^{(1)}, X_{11}^{(2)}, X_{22}^{(1)}, X_{12}^{(1)}, X_{12}^{(2)}, X_{21}^{(2)}, X_{33}^{(1)}, X_{33}^{(2)}-X_{32}^{(1)} X_{23}^{(1)}-X_{31}^{(1)} X_{13}^{(1)},\right. \\
& \left.X_{32}^{(1)} X_{23}^{(2)}+X_{31}^{(1)} X_{13}^{(2)}, X_{21}^{(1)} X_{32}^{(1)}-X_{31}^{(2)}, X_{32}^{(2)}, X_{22}^{(2)}\right) \text {, } \\
& \tau\left(C_{16}\right)=V\left(X_{11}^{(1)}, X_{11}^{(2)}, X_{22}^{(1)}, X_{12}^{(1)}, X_{12}^{(2)}, X_{21}^{(2)}, X_{33}^{(1)}, X_{33}^{(2)}-X_{32}^{(1)} X_{23}^{(1)}-X_{31}^{(1)} X_{13}^{(1)},\right. \\
& \left.X_{32}^{(2)} X_{23}^{(1)}+X_{31}^{(2)} X_{13}^{(1)}, X_{23}^{(2)}-X_{21}^{(1)} X_{13}^{(1)}, X_{13}^{(2)}, X_{22}^{(2)}\right) \text {, } \\
& \tau\left(C_{17}\right)=V\left(X_{11}^{(1)}, X_{11}^{(2)}, X_{22}^{(1)}, X_{22}^{(2)}-X_{21}^{(1)} X_{12}^{(1)}, X_{12}^{(2)}, X_{21}^{(2)}, X_{33}^{(1)}, X_{33}^{(2)}, X_{13}^{(1)}, X_{23}^{(1)}, X_{23}^{(2)}, X_{13}^{(2)}\right) .
\end{aligned}
$$

\section{Note que}

$$
\begin{aligned}
& \tau\left(C_{4}\right)=C_{14}, \\
& \tau\left(C_{5}\right)=C_{16}, \\
& \tau\left(C_{7}\right)=C_{7}, \\
& \tau\left(C_{8}\right)=C_{15},
\end{aligned}
$$




$$
\begin{aligned}
& \tau\left(C_{10}\right)=C_{12}, \\
& \tau\left(C_{11}\right)=C_{11}, \\
& \tau\left(C_{12}\right)=C_{10}, \\
& \tau\left(C_{13}\right)=C_{17}, \\
& \tau\left(C_{14}\right)=C_{4}, \\
& \tau\left(C_{15}\right)=C_{8}, \\
& \tau\left(C_{16}\right)=C_{5}, \\
& \tau\left(C_{17}\right)=C_{13} .
\end{aligned}
$$

\section{Finalizamos denotando}

$$
\begin{aligned}
& C_{18}:=\tau\left(C_{1}\right)=V\left(X_{11}^{(1)}, X_{11}^{(2)}, X_{22}^{(1)}, X_{22}^{(2)}, X_{21}^{(1)}, X_{21}^{(2)}, X_{33}^{(1)}, X_{33}^{(2)}, X_{32}^{(1)}, X_{32}^{(2)}, X_{31}^{(1)}, X_{31}^{(2)}\right), \\
& C_{19}:=\tau\left(C_{2}\right)=V\left(X_{11}^{(1)}, X_{11}^{(2)}, X_{22}^{(1)}, X_{22}^{(2)}, X_{21}^{(1)}, X_{21}^{(2)}, X_{33}^{(1)}, X_{33}^{(2)}-X_{32}^{(1)} X_{23}^{(1)}, X_{23}^{(2)}, X_{32}^{(2)}, X_{31}^{(1)}, X_{31}^{(2)}\right), \\
& C_{20}:=\tau\left(C_{3}\right)=V\left(X_{11}^{(1)}, X_{11}^{(2)}, X_{22}^{(1)}, X_{22}^{(2)}, X_{21}^{(1)}, X_{21}^{(2)}, X_{33}^{(1)}, X_{33}^{(2)}, X_{23}^{(1)}, X_{23}^{(2)}, X_{31}^{(1)}, X_{31}^{(2)}\right), \\
& C_{21}:=\tau\left(C_{6}\right)=V\left(X_{11}^{(1)}, X_{11}^{(2)}, X_{22}^{(1)}, X_{22}^{(2)}, X_{21}^{(1)}, X_{21}^{(2)}, X_{33}^{(1)}, X_{33}^{(2)}-X_{31}^{(1)} X_{13}^{(1)}, X_{13}^{(2)}, X_{23}^{(1)}, X_{23}^{(2)}, X_{31}^{(2)}\right), \\
& C_{22}:=\tau\left(C_{9}\right)=V\left(X_{11}^{(1)}, X_{11}^{(2)}, X_{22}^{(1)}, X_{22}^{(2)}, X_{21}^{(1)}, X_{21}^{(2)}, X_{33}^{(1)}, X_{33}^{(2)}, X_{13}^{(1)}, X_{13}^{(2)}, X_{23}^{(1)}, X_{23}^{(2)}\right) .
\end{aligned}
$$


APÊNDICE B 


\section{Referências Bibliográficas}

Atiyah e Macdonald (1969) M. F. Atiyah e I. G. Macdonald. Introduction to commutative algebra. Addison-Wesley Publishing Co., Reading, Mass.-London-Don Mills, Ont. Citado na pág. 14

Bourbaki (1980) Nicolas Bourbaki. Éléments de mathématique. Masson, Paris. ISBN 2-225-65516-2. Algèbre. Chapitre 10. Algèbre homologique. [Algebra. Chapter 10. Homological algebra]. Citado na pág. $18,19,20$

Bourbaki (1989) Nicolas Bourbaki. Algebra. I. Chapters 1-3. Elements of Mathematics (Berlin). Springer-Verlag, Berlin. ISBN 3-540-19373-1. Translated from the French, Reprint of the 1974 edition. Citado na pág. 8

Bruns e Herzog (1993) Winfried Bruns e Jürgen Herzog. Cohen-Macaulay rings, volume 39 of Cambridge Studies in Advanced Mathematics. Cambridge University Press, Cambridge. ISBN 0-52141068-1. Citado na pág. 18, 20

Cherednik (1989) Ivan Cherednik. Quantum groups as hidden symmetries of classic representation theory. Em Differential geometric methods in theoretical physics (Chester, 1988), páginas 47-54. World Sci. Publ., Teaneck, NJ. Citado na pág. 50

Colarusso e Evens (2015) Mark Colarusso e Sam Evens. Eigenvalue coincidences and $K$-orbits, I. $J$. Algebra, 422:611-632. ISSN 0021-8693. doi: 10.1016/j.jalgebra.2014.08.051. URL http://dx.doi.org/ 10.1016/j.jalgebra.2014.08.051. Citado na pág. 3, 45

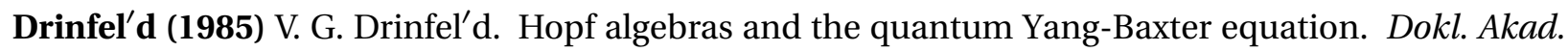
Nauk SSSR, 283(5):1060-1064. ISSN 0002-3264. Citado na pág. 50

Futorny e Ovsienko (2005) Vyacheslav Futorny e Serge Ovsienko. Kostant's theorem for special filtered algebras. Bull. London Math. Soc., 37(2):187-199. ISSN 0024-6093. doi: 10.1112/S0024609304003844. URL http://dx.doi.org/10.1112/S0024609304003844. Citado na pág. 1, 20

Futorny et al. (2005) Vyacheslav Futorny, Alexander Molev e Serge Ovsienko. Harish-Chandra modules for Yangians. Represent. Theory, 9:426-454. ISSN 1088-4165. doi: 10.1090/S1088-4165-05-00195-0. URL http://dx.doi.org/10.1090/S1088-4165-05-00195-0. Citado na pág. 1, 79

Gelfand et al. (1995) Israel M. Gelfand, Daniel Krob, Alain Lascoux, Bernard Leclerc, Vladimir S. Retakh e Jean-Yves Thibon. Noncommutative symmetric functions. Adv. Math., 112(2):218-348. ISSN 00018708. doi: 10.1006/aima.1995.1032. URL http://dx.doi.org/10.1006/aima.1995.1032. Citado na pág. 23, 24,25

Grivel (2004) Pierre-Paul Grivel. Une histoire du théorème de Poincare-Birkhoff-Witt. Expositiones Mathematicae, 22(2):145 - 184. ISSN 0723-0869. doi: http://dx.doi.org/10.1016/S0723-0869(04) 80010-0. URL http://www.sciencedirect.com/science/article/pii/S0723086904800100. Citado na pág. 12

Humphreys (1978) James E. Humphreys. Introduction to Lie algebras and representation theory, volume 9 of Graduate Texts in Mathematics. Springer-Verlag, New York-Berlin. ISBN 0-387-90053-5. Second printing, revised. Citado na pág. 8,11 
Kostant (1963) Bertram Kostant. Lie group representations on polynomial rings. Bull. Amer. Math. Soc., 69:518-526. ISSN 0002-9904. Citado na pág. 1

Kostant e Wallach (2006a) Bertram Kostant e Nolan Wallach. Gelfand-Zeitlin theory from the perspective of classical mechanics. I. Em Studies in Lie theory, volume 243 of Progr. Math., páginas 319-364. Birkhäuser Boston, Boston, MA. doi: 10.1007/0-8176-4478-4_12. URL http://dx.doi.org/10.1007/ 0-8176-4478-4_12. Citado na pág. 1, 2, 3, 39

Kostant e Wallach (2006b) Bertram Kostant e Nolan Wallach. Gelfand-Zeitlin theory from the perspective of classical mechanics. II. Em The unity of mathematics, volume 244 of Progr. Math., páginas 387-420. Birkhäuser Boston, Boston, MA. doi: 10.1007/0-8176-4467-9_10. URL http://dx.doi.org/ 10.1007/0-8176-4467-9_10. Citado na pág. 1, 2, 39

Matsumura (1970) Hideyuki Matsumura. Commutative algebra. W. A. Benjamin, Inc., New York. Citado na pág. $15,18,20$

Matsumura (1989) Hideyuki Matsumura. Commutative ring theory, volume 8 of Cambridge Studies in Advanced Mathematics. Cambridge University Press, Cambridge, second ed. ISBN 0-521-36764-6. Translated from the Japanese by M. Reid. Citado na pág. 20

Miščenko e Fomenko (1978) A. S. Miščenko e A. T. Fomenko. Euler equation on finite-dimensional Lie groups. Izv. Akad. Nauk SSSR Ser. Mat., 42(2):396-415, 471. ISSN 0373-2436. Citado na pág. 1

Molev et al. (1996) A. Molev, M. Nazarov e G. Ol'shanskiĭ. Yangians and classical Lie algebras. Uspekhi Mat. Nauk, 51(2(308)):27-104. ISSN 0042-1316. doi: 10.1070/RM1996v051n02ABEH002772. URL http://dx.doi.org/10.1070/RM1996v051n02ABEH002772. Citado na pág. 1

Molev (1997) Alexander Molev. Casimir elements for certain polynomial current lie algerbas. Em Group 21, Physical Applications and Mathematical Aspects of Geometry, Groups, and Algebras, Vol. 1, (H.-D. Doebner, W. Scherer, P. Nattermann, Eds), páginas 172-176. World Scientific, Singapore. URL http://www.maths.usyd.edu.au/u/alexm/publications/current.pdf. Citado na pág. 50

Molev (2007) Alexander Molev. Yangians and classical Lie algebras, volume 143 of Mathematical Surveys and Monographs. American Mathematical Society, Providence, RI. ISBN 978-0-8218-4374-1. doi: 10.1090/surv/143. URL http://dx.doi.org/10.1090/surv/143. Citado na pág. 23, 24, 25

Ovsienko (2002) Serge Ovsienko. Finiteness statements for Gelfand-Zetlin modules. Em Third International Algebraic Conference in the Ukraine (Ukrainian), páginas 323-338. Natsīonal. Akad. Nauk Ukraïni, İnst. Mat., Kiev. Citado na pág. 1

Ovsienko (2003) Serge Ovsienko. Strongly nilpotent matrices and Gelfand-Zetlin modules. Linear Algebra Appl., 365:349-367. ISSN 0024-3795. doi: 10.1016/S0024-3795(02)00675-4. URL http://dx. doi.org/10.1016/S0024-3795(02)00675-4. Special issue on linear algebra methods in representation theory. Citado na pág. $1,2,26,46$

Shepler e Witherspoon (2014) A. V. Shepler e S. Witherspoon. Poincare-Birkhoff-Witt Theorems. ArXiv e-prints. Citado na pág. 12

Vinberg (1990) È. B. Vinberg. Some commutative subalgebras of a universal enveloping algebra. Izv. Akad. Nauk SSSR Ser. Mat., 54(1):3-25, 221. ISSN 0373-2436. Citado na pág. 1

Zariski e Samuel (1975) Oscar Zariski e Pierre Samuel. Commutative algebra. Vol. II. Springer-Verlag, New York-Heidelberg. Reprint of the 1960 edition, Graduate Texts in Mathematics, Vol. 29. Citado na pág. 15,17

Želobenko (1973) D. P. Želobenko. Compact Lie groups and their representations. American Mathematical Society, Providence, R.I. Translated from the Russian by Israel Program for Scientific Translations, Translations of Mathematical Monographs, Vol. 40. Citado na pág. 22, 46 


\section{Índice Remissivo}

Álgebra

Afim, 16

Reduzida, 16

de Lie, 7

Abeliana, 7

Homomorfismo, 8

Linear $\mathfrak{g l}_{n}, 7$

Subálgebra, 8

Envolvente universal $U(\mathfrak{g}), 11$

Exterior $\bigwedge M, 10$

$n$-ésima potência $\bigwedge^{n} M, 10$

Filtrada, 6

Especial, 42

Graduada, 6

Homomorfismo, 6

Linear especial $\mathfrak{s l}_{n}, 8$

Linear geral $\mathfrak{g l}(V), 7$

PBW, 42

Especial, 42

Simétrica $S(M), 9$

Simplética $\mathfrak{s p}_{n}, 8$

Tensorial $T(M), 8$

Anel de coordenadas, 16

Aplicação

Multilinear, 5

Anti-simétrica, 5

Aplicação de Kostant-Wallach, 45

Aplicação parcial, 45

Fibra, 45

Fibra, 45

Caminho

Comprimento, 24

Simples, 24

Complexo, 17

de Koszul, 19

de uma sequência, 19

Homologia, 19

Conjunto algébrico, 14

Determinante quântico, 50

Dimensão

de Krull, 17 de uma variedade, 17

Espaço afim, 14

Filtração, 6

Associada

à álgebra envolvente universal, 13

à álgebra tensorial, 13

a Yangians, 50

Forma

Bilinear, 5

Não-degenerada, 5

Funções simétricas, 23

Funções simétricas completas, 23

Funções simétricas elementares, 23

Séries de potências de primeiro tipo, 23

Séries de potências de segundo tipo, 23

Graduação, 6

Associada

a uma álgebra filtrada, 6

Yangians, 50

Homologia, 17

Ideal

Associado, 15

Radical, 15

Interseção completa, 19

Matriz

Fortemente nilpotente, 46

Submatriz principal, 44

Quasideterminante, 23

Radical de um ideal, 15

Resolução de Koszul, 20

Sequência regular, 19

Subálgebra de Gelfand-Tsetlin $\Gamma$

Geradores, 22, 23, 25

para Yangians $Y_{p}\left(\mathfrak{g l}_{n}\right), 50$

Geradores, 50

standard de $U\left(\mathfrak{g l}_{n}\right), 22$ 
Teorema

da base de Hilbert, 14

de Ovsienko, 26

de Poincaré-Birkhoff-Witt

$\mathfrak{g l}_{n}, 14$

Yangians $Y_{p}\left(\mathfrak{g l}_{n}\right), 50$

dos zeros de Hilbert, 15

Topologia de Zariski, 16

Variedade

Algébrica, 14

Componente irredutível, 17

de Gelfand-Tsetlin

para $\mathfrak{g l}_{n}, 25$

para Yangians $Y_{p}\left(\mathfrak{g l}_{n}\right), 51$

Parcial, 45

Equidimensional, 17

Irredutível, 15

Yangians de nível $p, 49$ 